UCID -19952

DE84 Or4668

\title{
Electric Air Flltration: Theory, Laboratory Studies, Hardware Development, and Field Evaluations
}

W. Bergman, A. Biertiann, W. Kuhl, B. Lum, A. Bogdanoff, H. Hebard, M. Hall, D. Bariks, M. Mazumder and J. Johnson
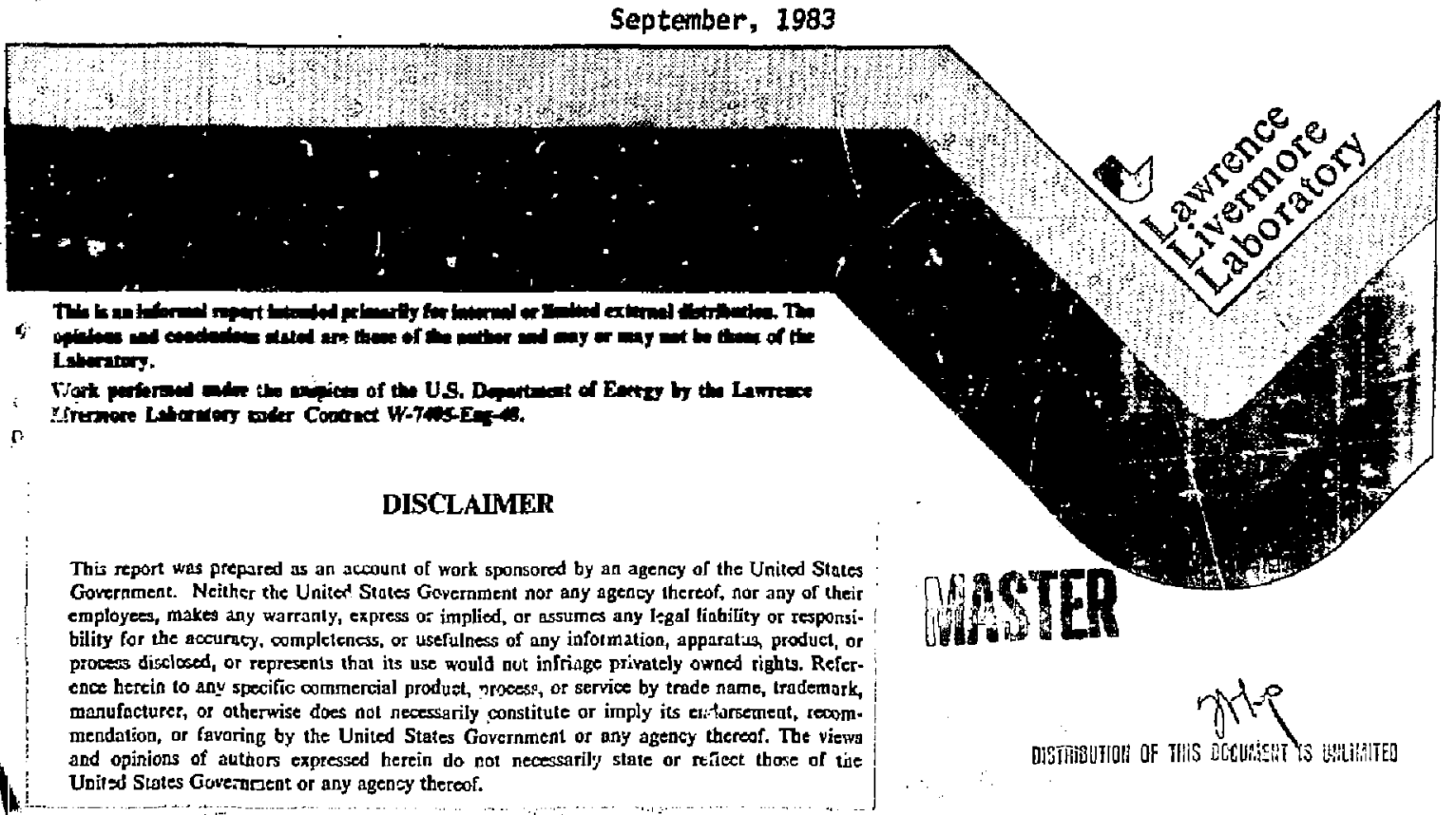
cnce hertin to any specitic commercial produch, jroess, or servioe by trade name, trademork, manufacturer, of otherwise does not necessirily constitute or imply its en-forsetneat, recom. mendatson, of favaring by the United States Government of any agency thereof. The viewa Unitsd States Gaveranent or any agency thereof.

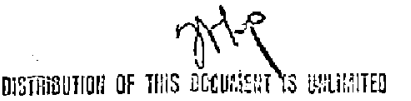




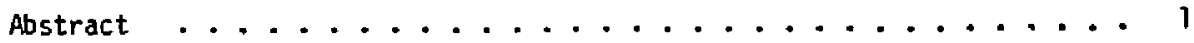

Executive Sumary ........................ 1

Present Filter-Test Methods ................... 7

Introduction ...................... 7

Present Filter-Test Hethods Based on Integrated Measurements

of He terodisperse Aerosols ................ 7

Theory of Present Filter-Test Methods .............. 9

DOP Filter-Certification Test and Filter Penetration ...... 11

Ow1 vs Laser Spectrometer Tests ... . . . . . . . . . . 13

Current DOP Test and HEPA Filter Penetration ............ 21

Development of Filter-Penetration Method as a Function of Particle Size 22

Introduction .................... 22

Test Aerosol Generation ...................... 23

Determination of Filter Penetratiori with Monodisperse

or Heterodisperse Aerosols ............... . . 24

Monodisperse Aerosols in Filter-Penetration Measurements . . . 27

"Quas i-Monodisperse" Aerosols . . . . . . . . . . . . 29

Monodisperse Aerosol Generators ............... 30

Particle-Size Measurement Shifts with Optical Counters . . . . 31

He terodisperse Aerosols and Filter Testing .......... 38

Computerized Filter-Test Facllity Description . . . . . . . . 42

Filter Penetration Test Instrument ............ 49

Differential Mobility Analyzer and Laser Particle Counter ..... 55

Dilution System for HEPA Filters ............... 63

Filter-Efficiency Measurements as a Function of Particle Size and Charge . 71

Introduction ....................... 71

Size-Charge Measurement by Mobility Fractionation . . . . . . . 72

Mathematical Derivation for Particle Charge . . . . . . . 74

Size-Charge Measurement by Laser Doppler Velocimetry ........ 81

Fundamental Studies of Electrically Enhanced Air filtration . . . . . 86

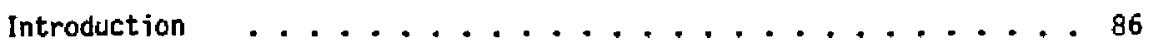

Background of Project .................... 87

Externally Charged Electric Air Filter . . . . . . . . . . . 90

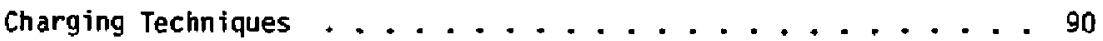

Enhanced Fllter Efficiency Experimental Results .........90 
Simplified ${ }^{-1}$ ter-Eifficiency Theories . . . . . . . . . 107

Stcady-State Penetration Theory ............. 107

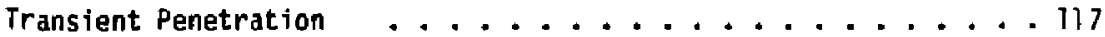

Rigorous Theory ....................... . 123

Permanently Charged Electric Air Filters ............ 125

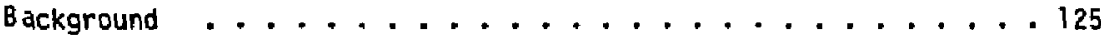

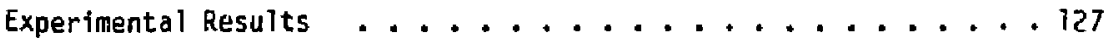

Aerosol Penetration as a Function of Particle Diameter . . . . 132

Techniques for Decreasing Filter Determination . . . . . . . 134

Effect of Particle Loading on Fijter Efficiency . . . . . . . 141

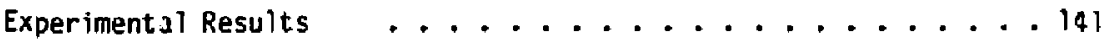

Horphology of Particle Deposits ............... 145

Simplified Particle-Loading Theory . . . . . . . . . 153

Development and Evaluation of Experimental Electric Air Filters . . . 166

Introduction $\ldots \ldots \ldots \ldots \ldots$

Design Criteria far Experimental Filters . . . . . . . . . 167

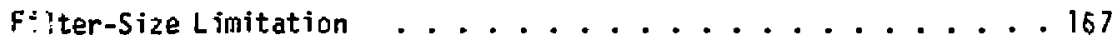

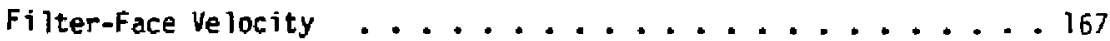

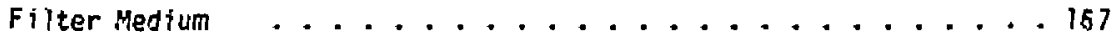

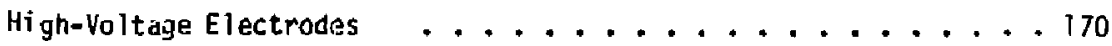

Filter Media with !ntegrated or Separated Electrodes . . . . 172

High-Voltage Power Supply . . . . . . . . . . . . 172

Cost/Benefit Analysis of Electric Air Filters ......... 176

Service Life of Prefilter-HEPA filter ............ 181

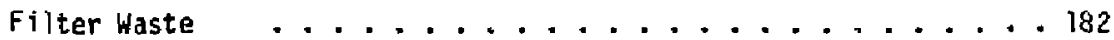

Accidental Radiation Exposure ............. 183

Electric Air Filters for Glove Boxes .............. . . 184

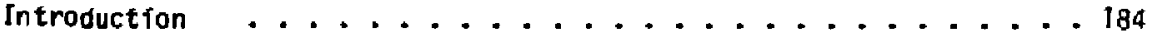

GloverBox Prefilter Using a Lever Arm . . . . . . . . . . 185

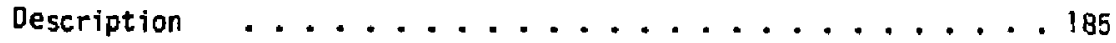

Laboratory Evaluation ............................

Field Evaluation in LLiNL's Uranium Powder Militing Box . . . . . 189

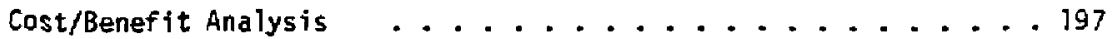

Gl ove-Box Prefilter with a Hinged Door ............ 201

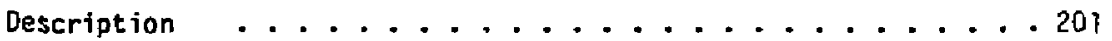

Laboratory Evaluation . . . . . . . . . . . . 204 
Field Evaluation in Pu-Dust Vacuum Box at Rocky Flats . . . . . 204

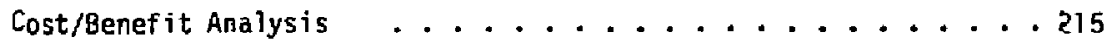

Electric Air Filters for Ventilation Systems . . . . . . . . . 220

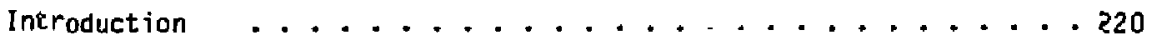

Ventilation Filter with Permanent Electrodes and Replaceable

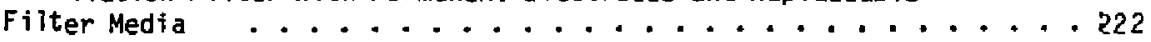

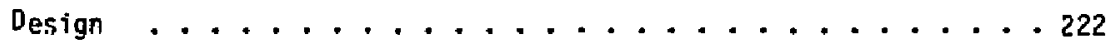

Laboratory Evaluation . . . . . . . . . . . . . . 222

Cost/Benefit Analysis . . . . . . . . . . . . . . 222

Disposable Filter with Electrodes Integrated with the Media . . . . 226

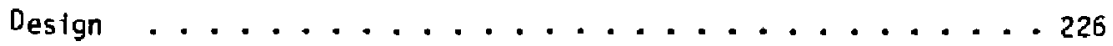

Laboratory Evaluation .................. 229

Field Evaluation of Disposable Electric Air Filter in

LLNL's Uranium Eurn Box ................. 229

Cost/Benefit Analysis .................... . . 233

Recirculating Electric Air Filter for Confined Spaces . . . . . . . ¿35

Introduction ...................... 235

Fundamental Concepts of Recirculating Air Filters . . . . . . 238

Initial Design and Laboratory Evaluation ........... 245

Redesigned Recirculating Air Filter ..............251

High-Temperature Electric Air Filter Using Stacked-Disk Design . . . . ?60

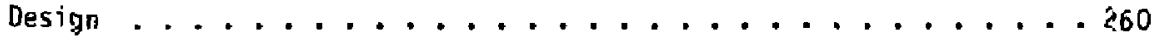

Laboratory Evaluation .... . . . . . . . . . . . . 264

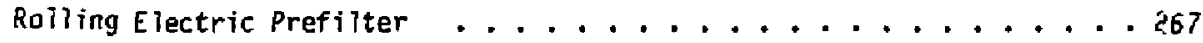

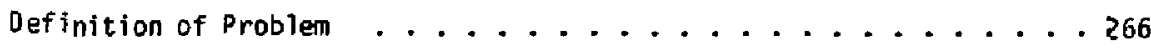

Feasibility Study of Rolling Electric Prefilter . . . . . . . 267

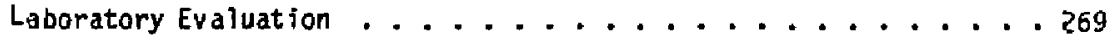

Multi-Path Roljing Electric Prefilter Desigr . . . . . . . . 274

Laboratory Evaluation . . . . . . . . . . . . . 280

Evaluation of Rolling Electric Prefilter in Dense Smoke Aerosols . . 282 
Electric Air Filtration: Theory, Laboratory Studies, Hardware Development, and Field Evaluations*

ABSTRACT

We summarize the results of a seven-year research project for the IJ. S. Department of Energy (DOE) to develop electric air filters that extend the service life of high-efficiency particulate air (HEPA) filters used in the nuclear industry. This project was unique to Lawrence Livermore Nationa 1 Laboratory (LLNL), and it entailed comprehensive theory, laboratory studies, and hardware development. We preserit our work in three major areas: (1) theory of and instrumentation for iilter test methods, (2) theoretical and laooratory studies of electric air filters, and (3) development and evaluation of eight experimentai electric air filters.

\section{EXECUTIVE SUMMARY}

The high-efficiency particulate air (HEPA) filters used in the nuclear industry to remove radioactive airborne contaminants are extremely effective and practical devices, and they will continue to be so despite a large number of new control devices presently available. HEPA filters, however, generate a significant volume of radioactive waste and are costly to purchase and operate. The actual cost of materials and labor to buy, change, test, and dispose of HEPA filters is several tines that of their initial purchase price. Moreover, valuable material trapped in HEPA filters cannot be recovered convenientTy.

In an effort to reduce the operational cost and the volume of radioactive waste that HEPA filters generate, the Airborne Waste Management Program Office of the Department of Energy's (OOE) Defense Nuclear Materials Program contracted with Lawrence Livermore National Laboratory (LLNL) to develop an enhanced filtration system that would extend the service life of HEPA filters and allow the recovery of radioactive materials. Our approach to solving this complex probiem cansisted of using electric prefilters to reduce the load on the HEPA

FThis work was performed under the auspices of the $U$. $S$. Department of Energy by Lawrence Livermore National Laboratory under contract No. W-7405-ENG-48. 
filters. We felt this approach had greater potential for successfully lengthening HEPA-filter life than other approaches in which scrubbers or electrostatic precipitators are used to reduce the load on the HEPA filter.

An electrified (charged or polarized) filter is an ideal candidate for removing sub-micron airborne particles in nuclear ventilation systems. Compared to a conventional fibrous filter, an electrified filter has a much higher efficiency and a significantly lower pressure drop at the same level of particle loading. This dramatic increase in filter performance due to added electrical forces has recently created "orld-wide interest in this method.

The concepts used $i$ our electric air filters are improvements in a relat:vely old filter technology. Electrostatics have been used to improve the performance of fibrous filters since 1930.' Although these filters can have a number of different configurations, all are based on either charging or polarizing of the filter fibers. An electrical force between the fibers and particles is generated, and this results in a significant increase in filter efficiency and filter life. The four primary methods of creating electric air filters include precharging the aerosols, polarizing the filter media with electric fields, combining these two methods, and permanently charging the fibers. We have not investigated prechargiag methods in our program because of the increased fire hazard of this method in nuclear applications.

The first of three major areas of this project was the development of new filter-test methods and instrumentation to provide needed experimental data on filter efficiency unavilable with existing tests and instrumentation. For example, we created three fully computerized filter-test apparatus (two small-scale with a 0-70 cfm flow and one large-5cale with a ,00-2000 cfm flow) for determining, ilter efficiency as a function of particle size from $0.01 \mu \mathrm{m}$ to $3.0 \mu \mathrm{m}$ diameter. (These routine filter tests are performed in less than ten minutes.) The computerized filter test apparatus also showed the inadequacy of the present test method used to certify HEPA filters. Our experimerics show that ten times more particles penetrate the HEPA filter than measured by the current dincty 1 phthlate (OOP) test. We recomend further development of our filter test method and instrumentation to replace the cirrent test.

Prompted by the strong effact of particle charge on filter efficiency, we also developed two devices that measure particle charge as well as particle size. One instrument that we developed at LLNL is based on a repeated sequerice 
uf separating the aerosols first according to electrical mobility and then measuring the size distribution of these aerosols. The other instrument, developed at the University of Arkansas, determines the size and charge of individual particles by monitoring the changes in particle motion in an oscillating electric field using laser doppler velacimetry. Both of these experimental instruments involve a complex operating procedure and are not developed sufficiently for routine measurements.

The second major emphasis in our project focused on fundamental studies that characterized electric air filters. We looked at theoretical modeling and smal1-scale laboratory experiments. The objective of our theory and laboratory experiments was to understand the mechanical and electrical filtration mechanisris for both clean and clogged (or loaded) filters in enough detail so that we could develop design criteria for experimental electric air filters. We conducted a wide and varied range of tests and calculations, a 11 of which helped establish determined criteria for experimental filters to be developed later on. We determined the effect of filter structure on filter efficiency and pressure drop, studied the efficiency of electric air filters using high-yaltage electrodes under a variety of operating conditions, and experimented with increased particle-laading on electric air filters.

New theories were developed that predict the two key features of electric air filters: the increased filter efficiency and the increased particle loading. The experimental and theoretical studies showed that the optimum electric filter design should have a minimum air velocity through the filter medium and use electrodes having a maximum $D C$ voltage and good electrical contact with ihe filter medium. We also determined that the optimum filter medium should have a minimum value of electrical conductivity, fiber packing density, compressibility and water adsorption.

The third and final area of our project was the development and evaluation of eight different electric air filters. The first two fijters were developed for use in glove boxes; one design was evaluated in a uranium powder box at LLNL and the other was evaluated in a plutonium dust box at Rockwell International, Rocky Flats Plant, Golden, Colorado. The electric air filter in the LLNL box extended HEPA service life eight times the usual amount and allowed for recovery of $70 \%$ of the uranium dust. The Rocky Flats design extended HEPA service iffe over 50 times and al lawed recovery of over $98 \%$ of the plutonium dust. 
The third and fourth experinental filters were designed for standard $2^{\prime} \times 2^{\prime}$ ventilation systems. One filter uses permanent electrodes with replaceable filter media while the other filter has a disposable filter with electrodes incorporated into the filter media. We evaluated the disposable elertric air filter in LLNL's uranium burn box. The filter showed an average efficiency of $98 \%$ with electrification and $91 \%$ without electrification. A durability evaluation in the uranium hurn box showed the necessity for cleaning the filte. whije it was in place in order to achieve the large extensions in HEPA service life and material recovery observed with the glove-box filters. we will recommend design changes in the filter and ventilation system in this report.

The fifth experimental electric air filter we developed was a recirculating air filter designed to increase ventitation in small, confined spaces. This experimental filter is extremely valuable in existing nuclear facilities where increasing the existing ventilation system would be prohibitively expensive. He aesigned and built several self-contained units that can be placed inside a confined space to provide additional air cleaning independent of existing air-cleaning systems. These units recirculate contaminated ir from a confined space through an electric air filter and blower and then ba-k into the same confined space.

We developed a theory of recirculating air filters to relate the roomcleaning efficiency to the size of the room, existing ventilation system, and the efficiency and flow rate of the recirculating air filter. Laboratory experiments conducted in a confined chamber using $\mathrm{MaCl}$ aerosols showed that the recirculating air filter reduced the aerosol concentration by $66 \%$ when using a 96\%-efficiant media and by $33 \%$ when using a $23 \%$-efficient nedia.

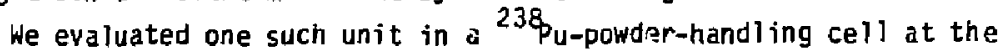
Savannah River Plant, Savannah River, South Carolina. Because of severe restrictions on the chemical contamination of the $\mathrm{Pu}$ powder. We could not use our standard glass-fiber media and used palypropylene-filter media instead. The results of the field evaluation showed that the ${ }^{236_{p_{u}}}$ powder which had been collected on the media had also melted the media. The high specific activity of ${ }^{238}$ pu raised the temperature cit the powder and sreated localized, high-temperature zones. The efficiency of the metted mediun was only $50 \%$ for the $\mathrm{PuQ}_{2}$ particles and the pressure drop increased to 0.6 inches. In our laboratory tests with $\mathrm{NaCl}$ aercsols, the same filter had an $85 \%$ efficiency and 
a pressure drop of 0.2 inches. Since we could not substitute high-temperature media in our recirculating filter because of chemical contamination, we had to design a new electric air fjlter.

Th is sixth experimental filter used a stacked-disk design with multiple disks fabricated from stainiess-steel filters and alumina-fibrous filters. Potential contamination from the alumina fibers was avoided by sandwiching the alumina media between the stainless-steel iilters and welding all of the edges. Laboratory tests of this design showed that the efficiency with NaCl aerosols increased from $77 \%$ to $99.9 \%$ when the filter was electrified. In addition to its high efficiency, this filter also can withstand high temperatures and rapid surges in air flow due to its rigid stainless steel construction. This filter is an ideal candidate for applications requiring a HEPA filter in high temperature, high pressure environments.

The seventh experimental filter we developed was a rolling electric prefilter for applications where high aerosol concentrations result in a rapid plugging of the HEPA $f i 7 t$ ter. Our evaluations, conducted in the ventilation duct of LLNL's large-scale fire test facility, showed the rolling prefilter was very successful in preventing HEPA plugging, a major accomplishment not achieved by any of the alternative air-cieaning devices evaluated so $f a r$.

Our eighth and final experimental filter was an electric YEPA filter for which we increased the ODP efficiency from $99.97 \%$ to $99.997 \%$ with an applied electric field of only $1.9 \mathrm{kV} / \mathrm{cm}$. Since the design of this experimental filter was $r$ ar from ideal, designs that we can optimize will yield significantly better results.

Al though the increased efficiency of the electric HEPA filter is more importani for clean-roon appiications than for nuclear applications, the potential for increased service life of electric HEPA filters is very attractive in nuclear applications. He anticipate that the electric HEPA filter wiil double the service life of a conventional HEPA filter based on similar studies with electric prefilters.

aic developod a quantitative method for determining the cost/benefit of using electric prefilters to extend the life of HEPA filters. Equations were developed relating the total cost and volume of waste to the filter, aerosol and operating variables for a single filter and for a prefilter-HEPA filter system. These equations were used to calculate the cost/benefit or electric and mechanical prefilters in the glove-box evaluations at LLNL and Rocky flats. 
The calculations show that electric prefilters extend the HEPA iffe by mare than four times the life extension achieved with identical mechanical filters. The two glove-box evaluations also showed that large extensions in HEPA 1 ife were obtained even for mechanical prefilters if the particle size is large. For example, the mechanical prefilter in the Rocky Flats evaluation extended the MEPA life by almost 30 times due to the large plutonium derosols (the activity median diameter was $3.5 \mathrm{um}$ ). In these applications, there is little motivation to have further extensions in HEPA life by using electric prefilters. The use of electric prefilters is most attractive in applications where particles are smaller than $14 \mathrm{~m}$ and, therefore, hard to remove by mechānical filters.

We will elaborate on the three project areas outlined above, looking first at the development of our filter-test methods and instrumentation, secondly at our various fundamental studies on electrically enhanced filtration, and lastly at our eight experimental electric air filters in some detail. 
PRESENT FILTER-TEST METHODS

INTRODICT ION

The experimental investigation of electric-filcration concepts requires measurement of filter efficiency and pressure drop at given air flows. Pressure drop depends primarily upon the velocity of the air flow through the fi?ter media and on media properties such as filter thickness and size, orientation, and packing density of the fibers. In ardition ti thesa parameters, filter efficiency also depends upon particle size and charge. Since many of these parameters vary significantly with different filter media and test condtions, we felt that as many parameters as possiti:e had to be measured in order to provide the best passible interpretation of test results. This knowledge was especially important for our eventual work with experimental electric air filters since we would need to know what parameters to change in order to improve a particular filter design.

Unfortunately, standard filter-test methods do not provide necessary information on the effect that impcrtant parameters, like filter size or packing density, have on filter efficiency. Current methods tend lo integrate individual parameters, such as particle size and clarge, into a sirije valur. thereby giving an average value, not a sperific one.

PRESENT FILTER-TEST METHODS BASEC ON INTEGRATED :IEMSUREMENTS OF HETERODISPERSE AEROSOLS

All of standard filter-test methods used by the U. S. and other countries are based, more or less, on a heterodisperse test aerosol to challenge the filter and integrated measurements of the sample aerosol concentration before and after the filter. Table 1 compares the four most widely used filter-test methods.

It is important to note that the same fiter tested with each of the four methods described in Table 1 has different penetration resuits, with the $U . S$. ASHRAE test yielding the lowes. penetration and the French uranin test the highes:. In general, for these four test methods, the smaller the particle size, the higher the penetration. This trend is illustrated in Fig. 1 where we have plotted the HEPA filter $f$ netration as a function of particle diameter 
Table 1. Comparison of the four major filter-test methods used wor ${ }^{\mathrm{d}} \mathrm{d}$-wids Eoday.

\begin{tabular}{|c|c|c|c|}
\hline Method & Test aerosol & $\begin{array}{l}\text { Volume average } \\
\text { diameter of aerosol }\end{array}$ & $\begin{array}{l}\text { Aerosol } \\
\text { measurement }\end{array}$ \\
\hline U. S. ASHRAE & $\begin{array}{l}\text { atmospheric dusi, } \\
\text { solid aernso? }\end{array}$ & 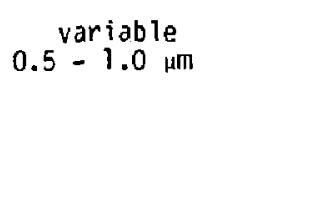 & $\begin{array}{l}\text { aerosols first } \\
\text { collected on filter } \\
\text { sample and measured } \\
\text { by optical } \\
\text { extinction of dirty } \\
\text { rilter }\end{array}$ \\
\hline British $\mathrm{NaCl}$ & $\begin{array}{l}\text { nebuli:ing } \mathrm{NaCl} \\
\text { water solution, } \\
\text { solid aerosol }\end{array}$ & $0.35 \mu \mathrm{m}$ & $\begin{array}{l}\text { flame photometer } \\
\text { measures aerosols } \\
\text { directiy }\end{array}$ \\
\hline U. S. DOP & $\begin{array}{l}\text { thermal-ginerated } \\
\text { DOP, liquid } \\
\text { aerDsol }\end{array}$ & $0.30 \mu \mathrm{m}$ & $\begin{array}{l}\text { light-scattering } \\
\text { photometer measures } \\
\text { aerosols directly }\end{array}$ \\
\hline French uranin & $\begin{array}{l}\text { nebu'izing uranin } \\
\text { water solution, } \\
\text { solid aerssol }\end{array}$ & $0.12 \mu \mathrm{m}$ & $\begin{array}{l}\text { aerosols first } \\
\text { collected on filter } \\
\text { sample; uranin } \\
\text { dissolved from } \\
\text { filter and measured } \\
\text { by fluorinetry }\end{array}$ \\
\hline
\end{tabular}

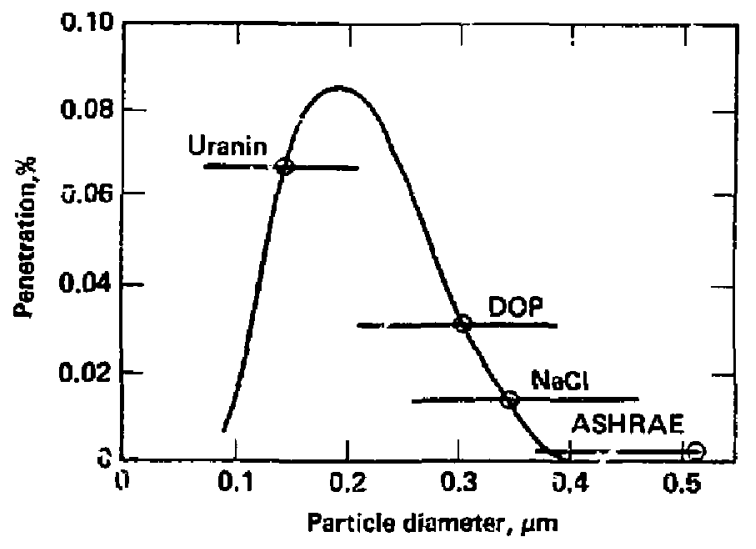

Fig. 1 Comparison of four major filter-test methinds showtig the portion of the filter-penetration curve measured by each technique. 
and have superimposed the approximate size ranges of the test aerosols used in the various filter-test methods. Note that the difference in the average particle size results in considerable variation in measured penetrations. Since the aerosois listed in Table 1 also have a broad range of particle sizes, filter-penetration tests thus represent, at best, an average penetration integrated over the particle-size range.

\section{THEORY OF PRESENT FILTER TEST METHODS}

The basic problem with present filter-test methods is that the measured filter penetration, $P_{M}$, varies with the number of particles in a given size $r$ ange, $r$, designated as $N(r)$ [or the particle-size distribution], and the instrument response function, $R(r)$.

In gener 1, the way in which the instruments in Table 1 respond varies approximately with the volume if particles, afthough the light-scattering photometer and the opticai-extinction meter depart the most from a true measurement of volume. The measurement of aerosols sampled before the filter, $M_{B}$, therefore, is an integated measureinent of the product of $N(r)$ and $R(r)$ :

$$
M_{B}=\int_{0}^{\infty} N(r) R(r) d i
$$

where $r$ is the particle size. This measurement of aerosols sampled before the filter is $i l l u s t r a t e d$ in Fig. 2, where we have $p$ lotted relative values of $N(r)$, $R\{r\}$, and their product as a function of $r$. Note that the instrument-response $f$ unction gives primary weight to the iarger particles in the tail of the particle distribution.

The measurement of aerosols sampled after the fitter requires an additiond factor to take into account that the filter has removed particles and thus has altered $N(r)$. The particle-size distribution of aerosols sampled after the filter is the product of filter penetration, $P_{F}(r)$, defined by the fraction of particles passing through the filter, ind the initial particle-size distribution of aerosols before the filter, $N(r)$. Figure 3 illustrates this type pf particie-size distribution. The measurement of aerosols sampled after the filter, $M_{A}$, is 


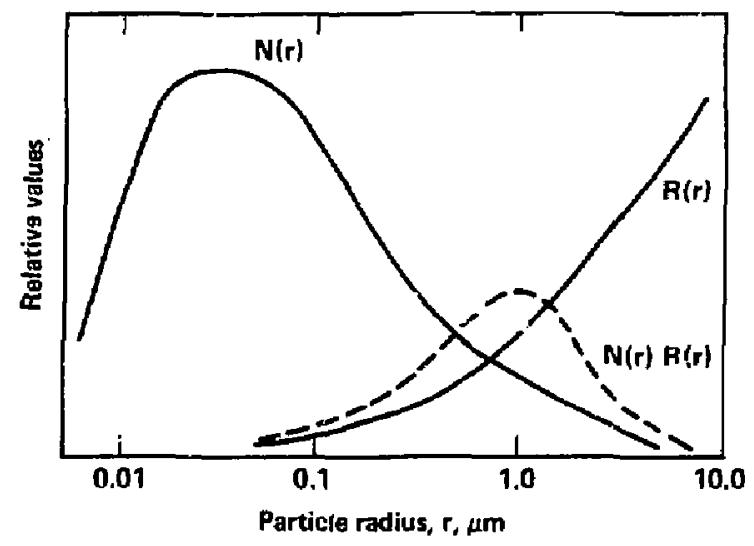

Fig. 2 The mezsurement of a typical neterodisperse aerosol with a single-valued detector (e.g., a photometer) equals the number of particles, $N(r)$, multiplied by the instrument response, $R(r)$, at each particle size, $r$, suminé over all particle sizes.

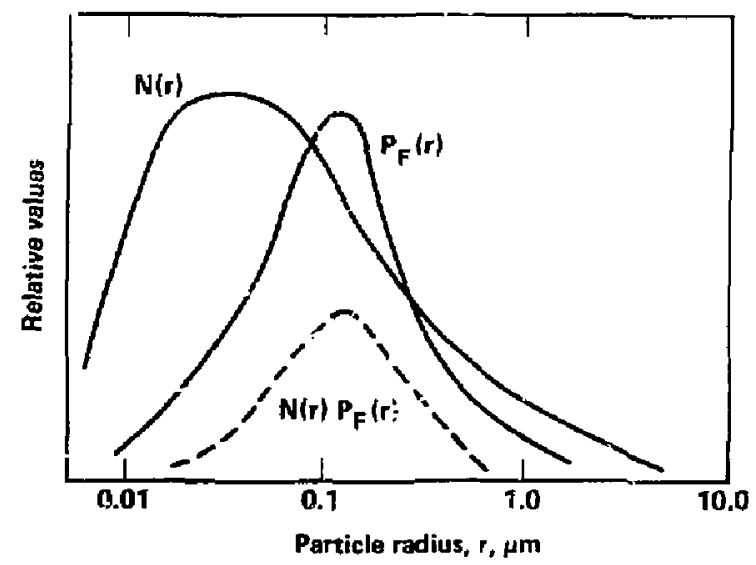

Fig. 3 The particle-size distribution of derosols sanpled after the filter equals the particle-size distributions before the filter. N(r), multiplied by the filter penetration, $P_{F}(r)$. 
$M_{A}=\int_{0}^{\infty} N(r) P_{F}(r) R(r) d r$

and is illustrated in Fig. 4 where we have plotted the curves for the particlesize distribution of aeroso?s after the $f: i$ ter, $N(r) P_{F}(r), R(r)$, the product of al1 three terms.

The complete filter-penetration measurement, $P_{M}$, is

$P_{i=1}=\frac{M_{A}}{M_{B}}=\frac{\int_{0}^{\infty} N(r) P_{F}(r) R(r) d r}{\int_{0}^{\infty} N(r) R(r) d r}$

The primary effect of a widely ranging particle-size distribution is an underestimation of the actual filter penetration, $P_{F}$, by the measured penetration, $P_{M}$, (or conversely, an overestination of the filter efficiency). We would like to be able to measure the filter penetration directly, and that is only possible if we have monodisperse particles. In that case, the siz? distribution function becomes a deita function, $\sigma\left(r-r_{0}\right)$, where $r_{0}$ is the monodisperse size.

In order to avoid this overestimation of filter efficiency in U. S. certification tests on HEPA filters, a large and complex vaporizer/condenser is used to generat monodisperse aerosols. Recent findings, however, have shown that UOP is not a monodisperse aerosol.

DOP Filter-Certification Test and Filter Penetration

Several investigators have found recentiy that DOP aerosois in the HEPAfilter-certification tests are not monodisperse $0.3-\mu \mathrm{m}$-diameter particles as had been assumed, but $r$ ather, heterodisperse aerosols with a count median diameter of $0.18 \mu \mathrm{m}$ and a genmetric standard deviation of 1.4. There was serious concern that, because of the heterodispersion of this aerosol, the measured vatues of filter penetration were underestimations of the true filter penetration $\left[P_{F}(0.3)\right]$. This concern was compounded when rejearchers also discovered that the "owl" particle-sizing instrument gave the same reading for a large number of different particle-size distributions.

The owl is an instrument that measures the size of monodispe"se aerosois by measuring the ratio of scattered light at two polarizations. Parameters 


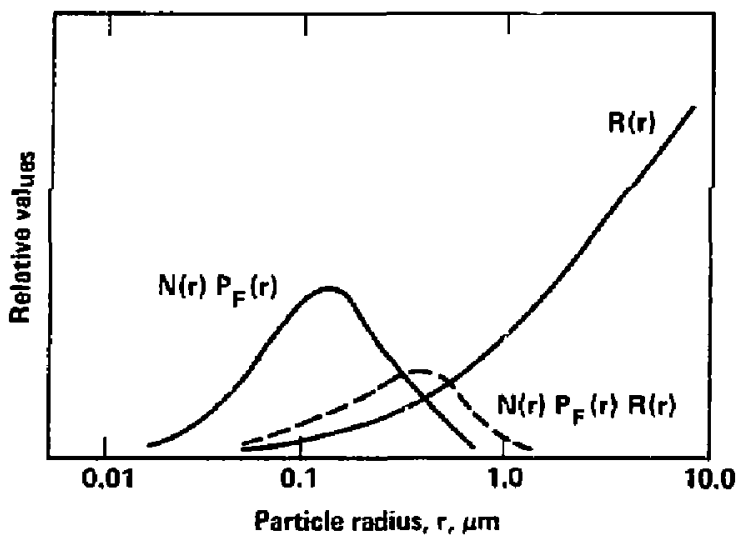

Fig. 4 The measurement of aerosols sampled after the filter with a single-valued detector (e.g., a photometer) equals the number of particles af ter the filter, $N(r) P_{F}(r)$, multiplied by the instrument response, $f(r)$, at each particle size, summed over all particle sizes. 
that control the DOP-aerosol generator are adjusted until the proper "owl" reading is obtained corresponding to $0.3 \sim$ m-diameter particles. A filterpenetration measurement can then be made by measuring an aerosol sample with a light scattering photometer betore and after the filter.

The owl's problem with the discovery that DOP aerosols are not monodisperse is its inability to uniquely define the particle-size distribution and the possible underestimation of the filter penetracion. This is iliustrated in Fig. 5 where three particle-size distributions yield the same owl reading. The broadest distribution in Fig. 5 is representative of the Dop aerosols used in HEPA-certification tests. Hinds et al. have determined that the ow 1 sees an average particle size weighted to the power $8.1^{2}$ :

$R_{o w 1}(r)=K_{1} \overline{r^{B .1}}$

where $R(r)=$ the 0 w 1 response to a particle of radius $r$ and $K_{1}$ is a proportionality factor.

Any number of particle-size distributions, like the ones in Fig. 5 that have the same weighted average size given by Eq. 4 , will yield the same owl reading. Thus, the validity of current OOP tests has been placed in question by the inability of the ow to uniquely measure the particle-size distribution.

\section{Ow1 Vs Laser Spectrometer Tests}

There is a concerted effort to replace the ow in the present oop test with ali instrument like the PMS-laser spectrometer, an effor: which we fael is unwarranted if filter penetrations are still measured with a light-scattering photometer. Independent tests with a PMS-laser spectroneter have shown that DOP aercsols have a broad range of particle-size distributions $* i$ th a significant number of particles exceeding $0.3 \mu \mathrm{m}$ in diameter. The concern is that these larger particles create decejvingly low HEPA-filter penetrations; a small increase in the size of DOF aerosois will result in a large decrease in filter penetration (Fig. ;). He fee? that replacing the owl with a laser spectrometer may create serious problems with filter-efficiency tests.

Our own analys is of the Dop filter tests has shcwn that the owl performs a complicated and necessary function in the filter test. The ow adjusts the 


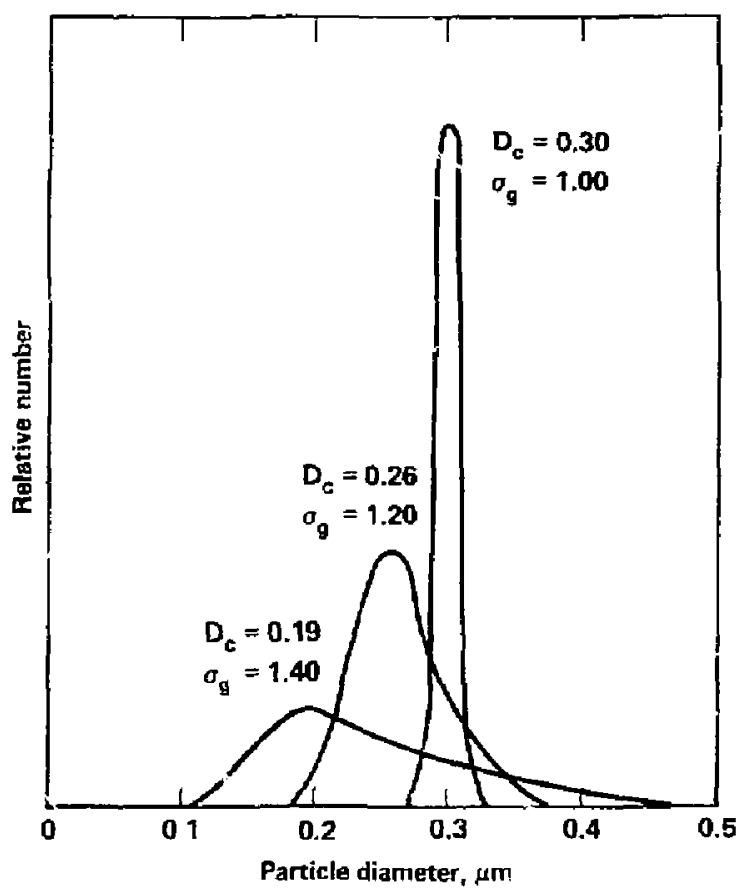

Fig. 5 Three different particle-size distributions that produced identical measurements of average size by the "owl." 
particle-size distribution so that the light-scattering photometer sees the heterodisperse DOP aerosol as monodisperse 0.3- $\mathrm{pm}$ miameter particles.

Figure 6 shows that the ow 1 and the photometer have a similar instrument response as a function of particle oiameter. Thus, as a first approximation, the photometer also sees the various particle-size distributions shown in Fig. 5 as monodisperse $0.3-\mu m-d i a m e t e r$ particles. This is only an approximation, and, as Fig. 6 shows, the two curves do not overlap exactly. The comparable equation for the photometer response is

$R_{p h o t}(r)=K_{2} \overline{r^{6.2}}$

where $k_{2}$ is a proportionality constant. This equation is valid from diameters of 0.1 to $0.4 \mu \mathrm{m}$. If the particles have $\mathrm{i} l 0 \mathrm{~g}$-normal distribution, then it is possible to calculate an equivalent monodisperse diameter that will give the same readings on either the priotometer or the owl. The equivalent. monodisperse diameters for the ow] and the photometer are given by EqS. 6 and 7 , respectively:

$\ln D_{\text {owl }}=\ln D_{\text {cma }}+4.05 \ln ^{2} \sigma_{\mathrm{g}}$ and

$\ln D_{\text {phot }}=\ln D_{\text {end }}=3.1 \ln ^{2} \sigma_{\mathrm{g}}$

where $D_{c m d}$ is the count median diameter and $\sigma_{g}$ is the geometric standard deviation of the particle-size distribution.

Equation 7 can be used to calculate equivalent monodisperse-particle diameters for heterodisperse aerosols as measured by tile photometer. Figure 7 shows the equivalent diameter plotted as a function of increasing heterodispersion for three cases. The two identified as $D_{\mathrm{cmd}}=0.3$ and $D_{\mathrm{cmd}}=0.2$ represent particle-size dis ributions in which the count median diameters are kept constant and the heterodispersion is allowed to increase. Wote that the equivalent photometer diameter increases significantly with increasing neterodispersion. The curve labeled ow $29^{\circ}$ represents a series of increasingly heterodisperse aerosols, as shown in Fig. 5, in which the owl sees an 


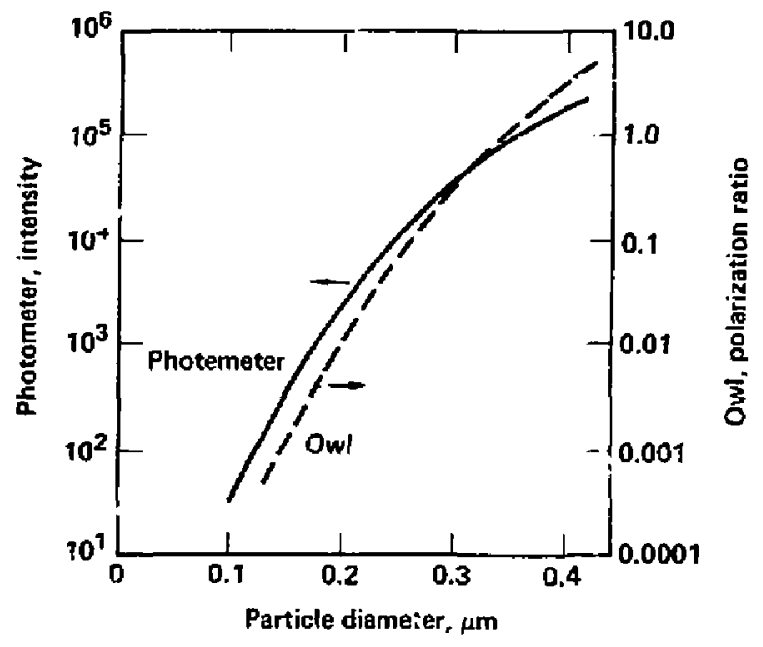

Fig. 6 Response of light scattering photometer and owl to different particle sizes. 


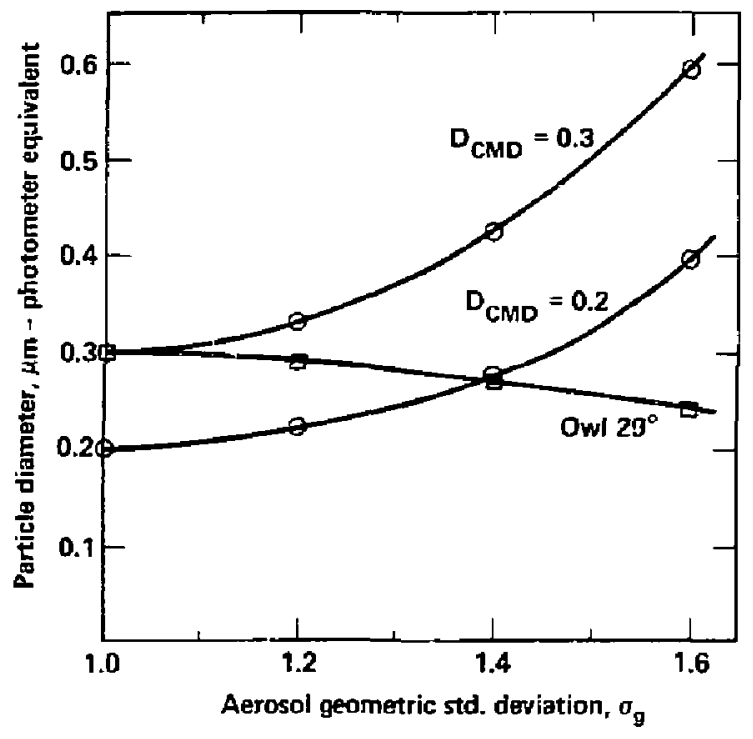

Fig. 7 The equivalent photometer diameter calculated from Eq. 9 for three different particle-size distributions as a function of incroasing heterodispersion. The particle-size distributions for two of the curves have a constant count median diameter, ${ }^{\circ} \mathrm{CMD}$, as the meterodispersion, ${ }^{\sigma} \mathrm{g}$, increases. The particle-size distribution for the owl with a $29^{\circ}$ reading is aetermined from Eq. 7 and shown in Fig. 5. 
equivalent $0.3-\mu m-d i a m e t e r$ aerosol as given by Eq. 6 . Note that the equivalent photometer diameter for a constant owl value decreases only slightly as the heterodispersion of the aerosols increases. Thus, we see that the owl provides an approximate measure of $0.3-\mu \mathrm{m}$ aerosol as seen by the photometer even for heterodisperse aerosals. We would therefore expect that the filterpenetration measurement using heterodisperse aerosols having a photometer equivalent diameter of $0.3 \mu \mathrm{m}$ would yield similar results to penetration meacrmements using monodisperse 0.3 -um derosols.

To test this hypothesis, we computed the peretration of a HEPA filter using Eq. 3 for various particle-size distributions, $N(r)$, all having the same owl reading. Particle-size distributions with various ${ }^{\prime}{ }_{c m d}$ and $\sigma_{q}$ were selected that had $D_{D W 1}=0.3 \mu \mathrm{m}$ in Eq. 6 . The filter-penetration finction, $P_{F}(r)$. used in these calculations is the solid curve stoment in Fig. 8 that.represents a least squares best fit of a log-normal distribution to the experimental measurements shown as triangles. The best fitting log-horinal curve is characterized by $Q_{\mathrm{cmd}}=0.143 \mu \mathrm{m}$ and $\sigma_{\mathrm{g}}=1.47$ The photometer response function $R(r)$ used in these calculations is given by $E q .5$ which is derived from data presented by Tillery et al. ${ }^{3}$ Figure 9 shows that increasing the heterodispersion of particle size distributions that have the same owl reading of 29 degrees (Fig. 5 shows three of these distritusions) causes only a small change in filter penetration. In contract, increasing the heterodispersion while maintaining a constant count medium diameter of 0.2 um causes a major shift in the filter penetratior. Tre purpose of measuring the particle size in DOP filter certification tests is to ensure that the filterpenetration measurements are made with DOP aerosols having an effective diameter of $0.3 \mathrm{\mu m}$. It is clear from Fig. 9 that the owl is better suited f $\mathrm{gi}$ ineasuring the effective particle size in the current fitter test than instruments like a laser particle-size spectrometer.

DOE is proposing a filter-test standard in which the cha'lenge aerosol is specified to have a $D_{c m d}=0.2 \mu \mathrm{m}$ and $a \sigma_{g}=1,4 . D O E$ is proposing that an aerosol-size spectrometer, either optical or electronic, be used for establishing these parameters. Although these aerosol-size spectrometers are $a b l y$ to uniquely define the particle-size distributions, they are unable to perform the function that the owl so simply and so elegantly performs.

Figure 9 shows that potentially serious errors in filter penetration could arise with the replacement of the owl by an optical spectrometer. Only when 


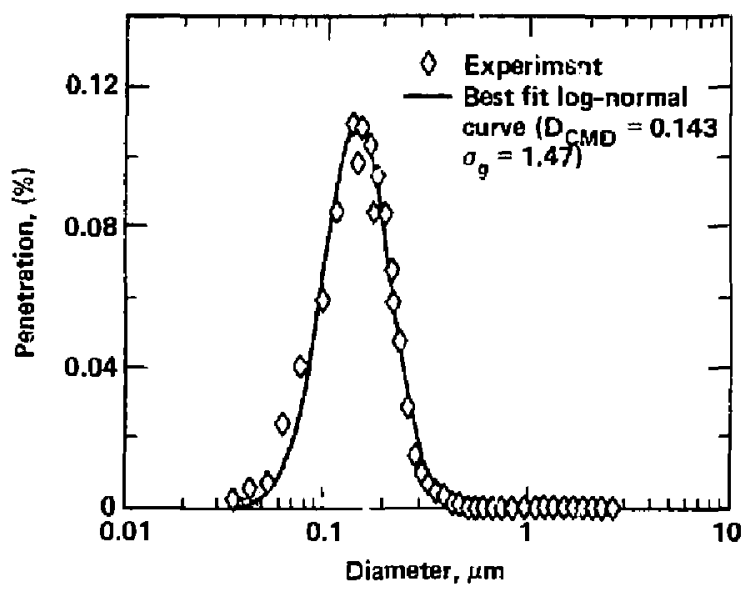

Fig. 8 Experimental penetration of a HEPA filter as a function of DOS particle diameter. 


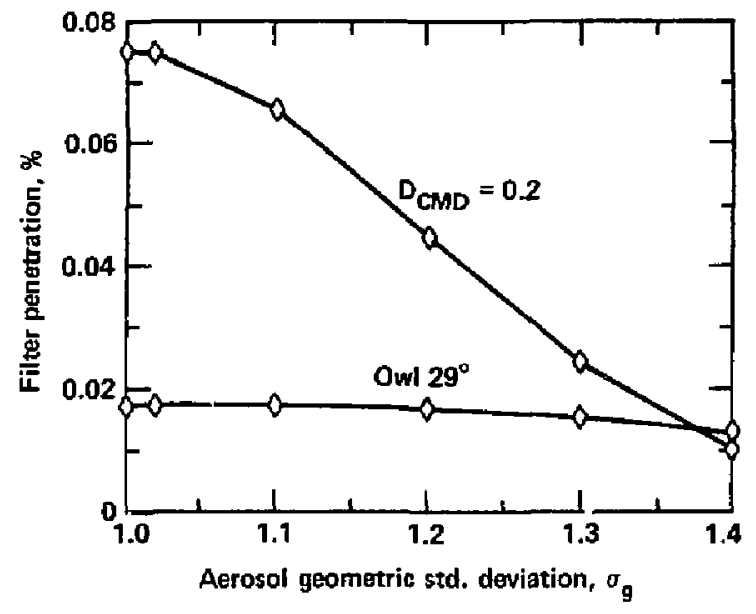

Fig. 9 Calculated $f i T$ ter penetration using Eq. 3 as a function of increasing heterodispersion for various particle size distributions, N(r). The curve for Owl $29^{\circ}$ was calculated for particle size distributions satisfying Eq. 6 with $D_{D_{W 1}}=0.3 \mu \mathrm{m}$. The curve for $P_{C M D}=D_{2}$ was calculated for particle

size distributions having a constant $\mathrm{D}_{\mathrm{CMD}}$. 
the $\sigma_{g}$ is within a narrow range of about 1.4 will the firter efficiencies outained with a photometer have results comparable to that of using an owl. Recognizing the potential error in specifying the size of the DOP aerosol with a laser particle-size spectrometer, the propesed doE fiiter-test standard will continue to use the owi for measuring the o0p particle size. However, to avoid the ambiguity in the owl measurement seen in Fig. 5, DOE is froposing that a laser particle-size spectrometer be used to restrict the particle-size distribution to that characterized by $D_{\mathrm{cmd}}=0.20 \pm 0.05$ and $\sigma_{\mathrm{g}}=$ $1.4 \pm 0.1$. As seen in Fig. 9, the owl and the laser particle-size spectrometer will yield the same filter-peivetration measurems: :s in this ragion.

The use of an aprospl-size spectrometer requires sifuting the concentrated a erosol to meet counting restrictions on 0 :tica! counters and mintenance requirements in electronic counters. The diiution requirement is the mast serious impediment to the use of a spectrometer since dilutors are notorious for losing the larger-sized particles. The loss of even a small fraction of the larger particle sizes would produce a major error in the photometer measurement since its response is heavily weighted toward the large particles. However, with proper calibration of the diluter, the measured particle-size distribution can be corrected for particle loss.

\section{CURRENT DOP TEST AND HEPA F ILTER PENETRATION}

The current test, as well as the proposed OOE method, for certifying HEPA filter greatly underestimates the penetration of aerosols through HEPA filters. The penetration of aerosois is more than ten times greater at $0.15 \mu \mathrm{m}$ than at the $0.3 \mathrm{um}$ diameter at which. HEPA filters ire testp. (Fig. 8). The selection of $0.3 \mu \mathrm{m}$ for testing HEPA filters was based on the theoretical and experimental findings of Langmuir and 8lodget ${ }^{4}$ forty years ago, wro showed that 0.3 r m $w$ as the most penetrating particle size. The concept of testing HEPA filters at their most penetrating particle size is highly desirabie since the filter penetration in any application will never exceed that determined in the certification test. Unfortunately, the current HEPA certification tes $\mathrm{i}$ falls far short of this goal. Moreover, recent findings by Lee and Liu ${ }^{5}$ have shown that the most penetrating particle size is not a constant, but varies with filter and operating variables. Th山s, it is apparent that without knowing the size of maximum penetration a priori, a filter-test proceaure must determine 
the penetration curve over a $r$ ange of particle sizes in order to determine the maximum penetration.

We developed a test for the routine measurement of filter penetration as a function of particle size as shuwn in Fig. 8. The test uses heterodisperse DoP a e: osols generated with a Laskin nozzel (or similar liquid), and a differential mobility analyzer coupled to a condensation nuclei counter and a lasar par'icle sounter as particie-sizing instruments. A dilution capability of 1000:] is required for the laser particle counter to permit ane particle to be counted at a time. A complete description of this system is given in the next section on filter penetration as a function of particle size.

We suggest that our filter-test system can serve as a model for developing a HEP. -filter certification test based on the measurement of maximum penetration. There are no major obstacies to the successfut implementation of our filter-test procedure. The aerosol generator, particle-sizing instruments, and dilutar are comercially availabie. We have already deve? nped the computer interface for rapid data acquisition and data reduction. Moreover, a routine filter test can be conductiod in less than two minutes if the primary interest in the filter test is the rejion of maximum penetration. The test resilts shown in Fig. 8 took 10 minutes in order to accumulate sufficient particle counts in the outer wings of the penetration curve. ' 2 believe that our filter test system can be successfully implemenced in $\mathrm{N}^{\mathrm{N}} \mathrm{f} f$. I ter-test $s$ cations.

OEVELOPMENT OF FILTER PENETRATION METHOD AS A FUNCTION OF PARTICLE SIZE

INTRODUCT ION

Al thoush filter-penetration measurements based on the integration over a broad range of particle sizes may be satisfactory for relative comparisons of filters, they are hard to interpret when exploring new filter concepts and developing prototypes. Representing the filter-penetration curve with a single $v$ alue is a gross simplification that higes impurtant capture mechanisms involved in aerosol filtration. Moreover, the curve is not a fixed one; it car shift to smaller or larger particle sizes, jecome braader or more compressed. and change dramatically in magnitude with changes in air velocity, silter thickness, fiber-packing density, fiber size and orientation, and external 
forces. In designing new filter media or experimental filters, it is critical to obtain the entire curve of filter penetration as a function of particle size.

We built a computerized filter-test facility to support our development of electric air filters. The unique features of this facility include a computerdiagnostic system and filter-test apparatus that can determine filter efficiency as a function of particle size for 0.01 to $3.0 \mathrm{ym}$ in diameter in addition to the stangard integrated efficiency measurements (this facjlity will be described in more detail later on).

TEST AEROSOL GENERATION

We used a variety of test aerosals, both monodisperse and heterodisperse, depending upon the test objective. Monodisperse aerosols are generally used for calibrating the particle-sizing instruments, but they aiso are used for measuring filter penetrations. The most frequently used monodisperse aerosols are Low Chemical's polystyrene latex (PSL) spheres that cover a diameter $r$ ange from $0.3 \mu \mathrm{m}$ to $2 \mathrm{um}$. (The lower size range is limited by debris aerosols in the residue while the upper size $r$ ange is limited by low particle concentration.) These monodisperse aerosols are generated by nebulizing a suspension of PSL spheres in water (alcohol may also be used for faster drying) with a wright-type nebulizer. Monodisperse aerosols of $\mathrm{NaCl}, \mathrm{DOP}$, and methylene blue in a $1.0 \mu \mathrm{m}-10 \mu \mathrm{m}$ diameter rangeare made with a vibratingorifice generator. A variety of liquid or solid monodisperse aerosols may be generated using this device provided that the material can dissolve in the solvent.

For monadisperse particles in the $0.01 \mu$ r. $\$ \mu \mathrm{m}$ diameter $r$ ange, we used a Thermal Systems, Inc., Model-307l, elect, istatic classifier. This instrument extracts a narrow range of particle sizes from a heterodisperse aerosol by passing the aerosol through a column with an applied electric field. At a fixed electric field, only those particles of a given size are extracted through a slit in the column. Smaller particles are deflected into the column wall before reaching the slit while larger particles are not sufficiently deflected and so pass by the slit. A variety of monodisperso solid and liquid aerosols car be generated by this procedure. Since the techniques for generating monodisperse aerosols described here are well- 
established and are fully documented elsewhere, we will not describe the details. 6,7

We generated heterodisperse solid aerosols of $\mathrm{NaCl}$ by nebulizing a $1 \%$ by weight solution of $\mathrm{NaCl}$ in water using one or more Wright nebulizers. To minimize the increase in the salt concentration due to evaporation, which increases the particle size, the solution is recirculated through a large reservoir. Heterocisperse liquid aerosols were generated using a Laskin nozzle generator in which compressed air is bubbled through the desired liquid. The a erosols are produced as the bubbles burst. Although any variety of liquids may be used in this generator, low-vapor-pressure oils like DOP are generally used to avoid the rapid evaporation of the small liquid particles. Bath of these heterodisperse aerosols have rather broad particle-size distributions characterized by mass median diameters of $0.8 \mu \mathrm{m}$ and geometric standard deviations of $2.0 .^{8}$

We chose not to use dust feeders in our filter tests even though a variety dust feeders are comerically availabie. Dust feeders generate heterodisperse a erosols that have mass median diameters greater than $5 \mu \mathrm{m}$ and these 1 arge particles sizes are far removed from the maximum filter penetration.

\section{DETERMINAT ION OF FILTER PENETRATION WITH MONODISPERSE}

OR HETERODISPERSE AEROSOLS

If monodisperse aerosols are used to measure the filter penetration curve, then a sequence of monodisperse aerosols is generated at increments of particle sizes sufficient to define the curve. Filter penetration is then obtained at each particle size by measuring the aerosol concentration before and after the filter using a single-valued detector (Fig. 10). A variety of aerosol generation techniques and particle-measuring instruments can be used with monodisperse aerosols.

However, if heteradisperse aerosols are used, the filter-penetratian curve can be obtained in a single step by measuring particle concentratiori as a function of size before and after the filter using an aerosol spectrometer. Figure 11 illustrates the key features of this approach. Various techniques for generating heterodisperse aerosols and measuring the particle-size distribution can be used in this situation. 


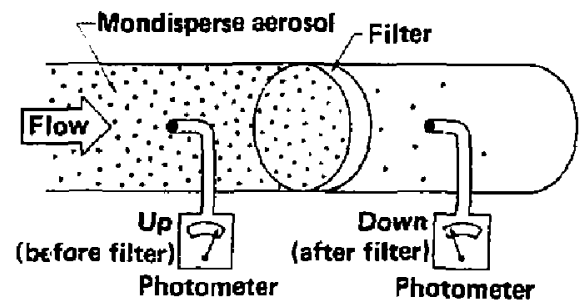

Fig. 10 Schematic showing the key components of a filter-test method using monodisperse aerosols and a single valued detector. 


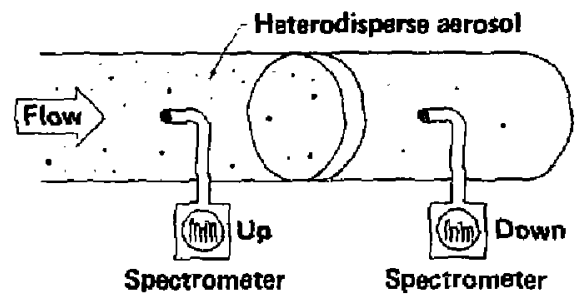

Fig. 11 Schematic showing the key component; of a filter-test method using heterodisperse aerosots and a particle-size spectrometer. 
Theoretically, the determination of filter penetration as a function of particle size, $P_{F}(r)$, is identicai for both test methods. However, the requirement of the partirle-size distribution to become a delta function $\delta\left(r-r_{0}\right)$ at each size $r_{0}$ experimentally is accomplished by the aerosol generator in the monodisperse case, and by the aerosol-size spectrometer in the heterodisperse case. A comparison of the two methods for measuring fitter penetration is summarized in Table 2.

In making an overall camparison between filter-test methods based on monodisperse and heterodisperse aerasols, we view the monodisparse method as labor-intensive and the heterodisperse method is hardware-intensive. The sequencing of a series of monodisperse aerosol generators followed by filterpenetration ineosurements is extremely time-consuming and does not readily lend itself to automation. In contrast, aerosol-size spectrometers automatically measure the aerosol concentration as a function of particle size. Taking the $r$ atio of these measurements of samples collected before and after the $f i l$ ter provides direct filter-penetration measurements. We shall discuss this idea further now.

\section{Monodisperse Aerosois in Filter-Penetration Measurements}

For a filter-test method using monodisperse aerosols, the generation of aerosols has a number of problems that make this approach unsuitable for determining filter penetration as a function of particle dianeter from 0.01 to $3.0 \mathrm{um}$. As shown in Table 2, the use of monodisperse aerosols for determining filter-penetration curves requires multiple generators, has a low output concentration, and is not monodisperse over the entire particie-size $r$ ange. Multiple generators are required to produce manodisperse aerosols at sufficient size increments to cover the range from 0.01 to $3.0 \mu \mathrm{m}$. This requirement not only adds to the complexisy of this filter-test method, but it aiso makes this method extremely time-consuming since each aerosol generator requires several minutes to stabilize once is has been turned on. Even if ail of the aerosol generators are operating continuously while the output of only one generator is used at a time, the time required to purge aerosols from the filter-test apparatus, sampie 1 ines, and detector for each new aerosal adds appreciabiy to the total test time. 
Table 2. Comparison of filter-penetration measurements from 0.01 to $3.0 \mathrm{um}$ particle diameter using heterodisperse or monodisperse aerosols.

$\begin{array}{lll}\text { Instrument } & \text { Monodisperse } & \text { Heterodisperse } \\ \text { Aerosol generator } & \begin{array}{l}\text { o multiple generator } \\ \text { o low concentration } \\ \text { o not monodisperse } \\ \text { over ent ire range }\end{array} & \begin{array}{l}\text { o single generator } \\ \text { o high concentration }\end{array} \\ \text { Aerosol detector } & \begin{array}{l}\text { o one or more single- } \\ \text { valued detectors }\end{array} & \begin{array}{l}\text { o two aerosol-size } \\ \text { spectrometers }\end{array} \\ \text { Penetration measurement } & \begin{array}{l}\text { two-s tep method: } \\ \text { aerosol size is fixed } \\ \text { by generator and then } \\ \text { concentration is } \\ \text { measured by detector }\end{array} & \begin{array}{l}\text { one-step method: } \\ \text { aerosal size and } \\ \text { concentration measured } \\ \text { directiy by } \\ \text { spectromater }\end{array} \\ & \text { o time-consuming } & \text { fast }\end{array}$


Monodisperse-aerosol generators also have a low output concentration that. limits their application in filter tests to those filters having efficiencies below 99\%. This limitation is due to the insufficient number of particles penetrating the filter for a concentration measurement. The major problem with monodisperse aerosols, however, is that they are not monodisperse over the particle-size range from 0.01 to $3.0 \mu \mathrm{m}$ in diameter,

Any of the techniques for generating monodisperse aerosols described earlier can be used for determining filter penetration as a function of particle size. These techniques include the use of PLS, electrostatic classifiers, and vibrating-orifice generators (VOG). Another technique that is frequently classified as a monodisperse generator is a condensation jeroso? generator for producing low-vapor-pressure liquid aerosols. The oop generators used in certifying HEPA filters belong to this class of aerosol generators. Again, as mentioned above, these condensation aerosols are not truty monodisperse and introduce an error in the filter-penetration measurements.

"Quasi-Monod isperse" Aerosols

Lee and Liu measured filter-penetration curves using a condensation DOPaerosol generator as a source of "quasi-monodisperse" aerosols covering a range of particle sizes from $0.035 \mu \mathrm{m}$ to $1.3 \mu \mathrm{m}$ in diameter. ${ }^{9}$ Particle size was contralled by the concentration of DOP in alcohol solutions which varied from $0.005 \%$ to $100 \%$. In this technique, relatively monodisperse $00 \mathrm{P}$ aerosols were produced by atomizing a given solution, heating the resulting heteroaisperse aerosols unti] they vaporized, and then rapidly cooling the vapor to form "quasi-monodisperse" aerosols. Lee and Liu have used 11 different solutions to cover the particle-size range. Al though these aerosols have relatively narrow size distributions, they still have significant geometric standard deviations ranging from 1.69 at $0.035 \mu \mathrm{m}$ to 1.15 at $1.3 \mu \mathrm{m}$ diameter. It is noteworthy that Lee and Liu observed $\sigma_{\mathrm{g}}=1.37$ at $0.3 \mu$ m diameter which is nearly identical to the values observed for DOP aerosols in the DOE filter-test-certification laboratories. They used an comercially available electrical-aerosol detector to detect aerosol concentration before and after the filter for penetration measurements. This instrument had a response function that increased approximats?; lineariy with particle size. 
There are three major problems with Lee and Liu's approach for determining $f i 1$ ter-penetration curves. First, the aerosols are not monodisperse and so present problems similar to those described in the previous section on the pop HEPA-filter-certiication test, albeit not as serious. The reason heterodispersion has a lower effect in Lee and Liu's approach is that the electrial detector has a much lower dependence on particle size than does the optical photometer. (This effect will be discussed in greater detail in the section on aerosol detectors.) Ironica $13 y$, the use of monodisperse aerosols in $f i$ ter tests also requires particle-sizing instruments to establish the size of the "quasi-monodisperse" aerosol, thereby eliminating the need for monodisperse aerosols. In the DOP HEPA-filter-certification tests, an "ow]" is required to determine the size of the DOP aerosols, Dut it cannot be used for penetration measurements.

A second problem with the "quas j-monodisperse" aerosol generator is the limitation of particle sizes to less than $1.3 \mu \mathrm{m}$ in diameter. This is only a serious problem for low-efficiency filters operating at low air flows where much of the penetration curve falls beyond $1.3 \mu \mathrm{m}$ in diameter.

The tnird and perhaps the most serious problem is the excessive amount of time required to generate a stable monodisperse derosal and to sample before and after the filter for each of the different size aerosols. Monodisperse aerosol generators suffer from low concentration and background heterodisperse aerosols.

Monodisperse Aerosol Generators

Except for the condensation aerosols discussed above, the generation of "monodisperse" derosols over the size range of 0.01 to $3.0 \mu^{m}$ in diameter requires at least two different types of generatars because of the 1 imited size range of each generator. An electrostatic classifier is needed to produce monodisperse aerosols in the 0.01-0.1 $\mathrm{m}$ dianeter particle-size range; it does this by extracting a slice from a heterodisperse aerosol source. Above $0.1 \mu \mathrm{m}$, the classifier produces increasingly heterodisperse aerosols because of the unavoidable multiple charges on the aerosols. Siace the electrostatic classifier extracts a sample fru a heterodisperse aerosol, a variety of solid or liquid aerosols may be generated. The electrostatic classifier is a convenient source of monodisperse aerosols from 0.07 to $0.1 \mu \mathrm{m}$ in dianzter 
since the selected size can be quickly changed by electronic means.

Un fortunately, the output concentration is so low that only relatively poor filters can be tested and only at small flow rates to minimize the dilution effect. The extreme effect of this dilution is iliustrated by diluting the 1 liter/min output from the classifier with 28,300 liter/min in a typical filter test.

PSL aerasais can be used as a saurce of monodisperse aerosols from 0.3 to $3.0 \mu \mathrm{m}$ in diameter. But, although the spheres themselves are monadisperse, the aerosol generated from the latex suspension is not. Figure 12 shows the size distribution of the aerosol generated from the atomization of $0.796-\mu \mathrm{m}$ diameter latex spheres in a water suspension. As seen in Fig. 12, the aerosol has a very broad size distribution with a logarithmically increasing concentration with decreasing particle size and a sharp spike that represents the latex spheres. The large increase in particle concentration below the $0.5-\mathrm{m}-$ aiameter $r$ ange is produced primarily from the agents used to stablize the iatex spheres. Al though these background aerosols can be reduced by varjouc. techniques, they still dominate the smaller particle sizes and thur limit the use of latex spheres to particle sizes above $0.5 \mu \mathrm{m}$ in diameter.

figure 12 also shows a second but much smalier spike at $1.0 \mu \mathrm{m}$ that represents sphere doutlets. Sphere doublets and triplets are produced when the latex suspension is too concentrated and more than one sphere is present in the atomized droplets. Al though sphere doublets are a potential source of error when using iatex spheres as sources of monodisperse aerosols, this problem is easily corrected by restricting the concentration of latex in the suspension. Unfortunately, limiting the latex concentration in the suspension results in a low output concentration that limits the use of these aerosols to low efficiency filters.

\section{Particle-Size Measurement Shifts with Optical Counters}

The use of monodisperse latex spheres in filter tests recently : ecomended by Davies requires a particle-size analyzer such as an optical particle counter coupled with a pulse-height analyzer. ${ }^{10} \mathrm{~A}$ single-valued detector that is sensitive over the entire size range of interest cannot be used because of the presence of a large concentration of small background aerosols. Even detectors 1 ike light-scattering photometers that are more sensitive to larger particles 


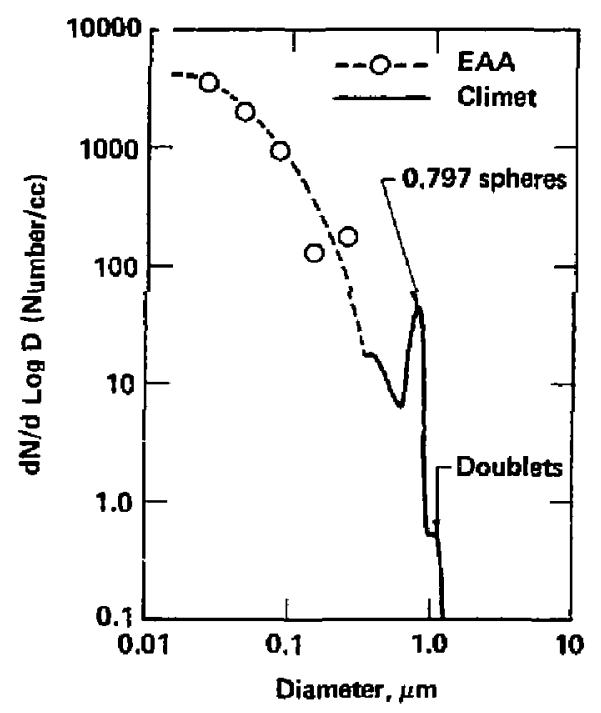

Fig. 12 Particle-size distribution generated from atomizing a suspension of $0.797-4 m$ latex spheres. 
cannot be used for latex spheres below $1.0 \mu \mathrm{m}$ because of the interference from the background aerosols. Although the photameter response to the small background aerosols is very small, the large numbe" of these aerosols results in a significant signal and produces an errcneous filter penetration.

Although the use of monodisperse latex spheres at firsi glance appears to be an attractive method for $f i l$ ter-penetration measurements, the requirement of a particle-size analyzer leads to the ironic conclusion that monodisperse latex spheres are no longer necessary. Any heteradisperse aeroscls could be used with the added benefits of a single derosol generator which can produce a high concentration of aerosols.

There are additional problens with the use of optical particle counters for measuring filter-penetration of latex spheres. These problems stem from the inabijity of optical particle counters to measure narrow size distributions accurately. Monodisperse latex particles may be counted in larger or smaller size channeis due to any one of several reasons, including 1 imitations in electronic-signal processing, coincidence, and interference from uncountable particles. These are general limitations and may apply both to classic optical counters as well as to laser optical counters. Small shifts in the measured particle size due to any of these problems can result in large errors in the calcuiated filter penetration.

A potential cause of particle-size shifts is a shift in the signal baseline voltage that may occur at high particle-count rates. Although this shift in the baseline signal is extremely small and is barely noticeable for even monodisperse aerosols, the shift can create major problens in filterpenetration measurements. Figure 13 shows the particie-size distribution of aerosols generated by nebulizing a water suspension of 0.797 pm-diameter latex spheres; the higher-concentration latex sample is shifted toward smaller particle sizes. Major errors in filter penetration occur as a result of these shifts (Fig. 14).

We trjed to compensate for the experimental shift in the particle size by artificially shifting the upstream size distribution curve unil the two latex peaks matched at $0.742 \mu \mathrm{m}$. The recomputed percent penetration as a function of particle diameter is shown as the dotted curve in Fig. 14. A) though the corrected penetration curve is much better, it still shows significant fluctuations. Figure 14 also shows the penetration curve when using heterodisperse DOP aerosols using both a Thermo Systems, [nc. electrical aerosol 


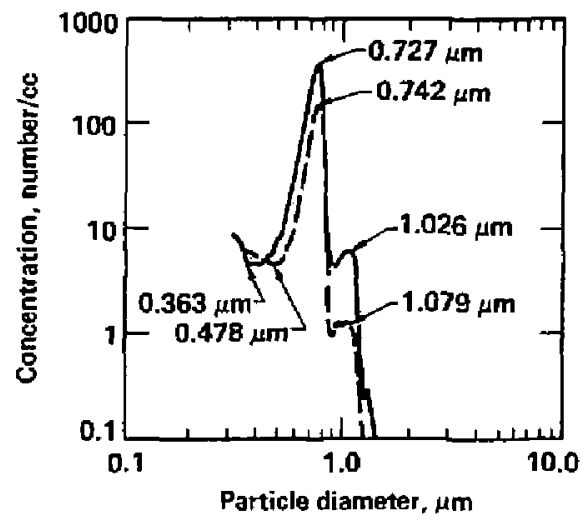

Fig. 13 Particle-size distribution of $0.797-1 \mathrm{~m}$ diameter latex aerosols showing minor size shifts before and after the filter. Solid curve represents particle-size distribution before a filter; dashed after. 


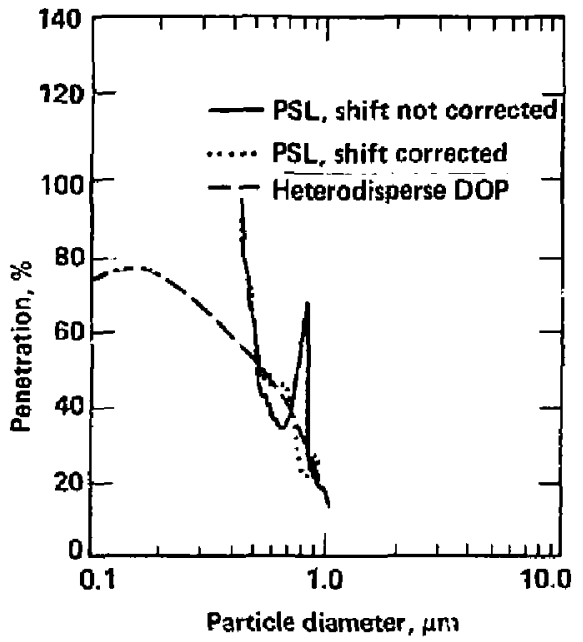

Fig. 14 Filter penetration calculated from the concentration measurements in Fig. 13 for the case where no correction was made for the shift in size (solid curve) and for the case where a correction was made (dotted curve). The dashed curve shows the penetration for heterodisperse DOP aerosols as a reference. 
analyzer and tive same Climet optical countar. Note that the heterodisperse DOP aerosol did not show the same erratic behavior as occurred with monodisperse PSL. Al though shifts in particle size most likely occurred for the heterodisperse UOP aerosols also, the gradual change in particle concentration with size minjmized any errors in the calculated penetration.

Another potential source for a shift in particle-sjze distributions for optical particle counter is due to coincidence whenever two or more particles intersect the lijht badm at the same time. When this occurs, ine particle counter does int "see" two smaller sized particles, but rather, a single, large particle. The effect of coincidence on the measured particle-size distribution is tu rejuce the number of small particles and increase the number of large particles.

We illustrates this trend in Fig. 15 where we plotted tre particie-size distributions for threg different concentrations of the same heterodisperse $\mathrm{NaCl}$ aerosol. The concantrations were yaried by diluting the aerosol with filtered air. We abtained these data with the laser particle counter, Model ASAP-300, from Particle Measuring Systems, Inc. Note that as the aerosol conceniration increases, the size distribution changes dramaticaily from a decreasing concentration with size to an increasing concentration with size. At intermediate concentrations, the aerosol appears to have a maximum concentration arounc $0.25 \mu \mathrm{m}$ in diameter. Such major changes in the particle-size distribution would lead to ser jous errors in filter-penetration measurements even whan using heterodisperse aerosols. However, even slight changes in the particle-size distribution due to coincidence would lead to major errors when using monodisperse arrosols.

Another potential cause for shifts in particle-size measurements with optical particle counters is due to the generation of countable pulses by nigh concentrations of non-countable, undersized particles. Whitby and Liu have shown that the size of PSL particles will shift to large channels when there is a large concentration of particles below the minimum detectable size of the particle counter. 13 They have shown that $0.9-\mu \mathrm{m}-\mathrm{d}$ iameter latex spheres will be shifted to $1.05 \mu \mathrm{m}$ at moderate concentrations and to $1.5 \mu \mathrm{m}$ at $\mathrm{high}$ concentrations of non-countable aerosols. This shift presents a major problem

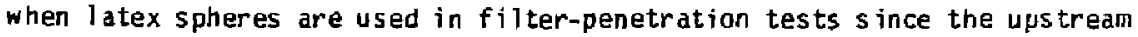
particle measurement will generally be shifted to a greater extent than the downs tream meas urement. 


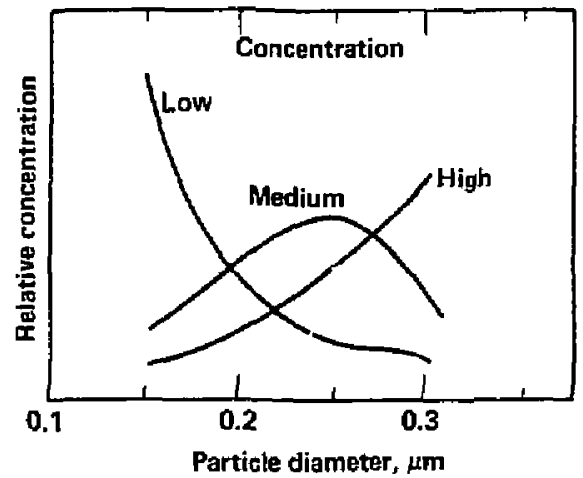

Fig. 15 Changes in particle size distribution measured with a laser particle counter when the same aerosol is measured at increasing concentrations. The drastic changes in size distribution are due to coincidence errors where more than one particle is counted at a time. 
A filter will generally remove a large fraction of the non-countable aerosols due to the difrusion capture mechanism, thereby minimizing any shift in the downstream size measurement. Large errors in filter penetration are made when comparing the upstream and downstream concentrations since the latex peaks do not fall in the same size channels. Figure 14 illustrates the large fluctuation in filter penetration that can occur with even a small shift in the size measurement when using monodisperse aerosols. However, if heterodisperse aerosals are used, then only a minor error in $f i l$ ter penetration is made. ih is follows because the concentration varies slawly with size and any shift will result in only minor changes in concentration.

\section{Heterodisperse Aerosols and Filter Testing}

Although problems with monodisperse aerosols can be minimized, heterodisperse aerosols are better for filter testing. The error in filter penetration due to the shift in particle size by any of the above three mechanisms can be minimized by using extrenely dilute concentrations of aerosols or by integrating the particle counts over a wide range of particle sizes. In $f i]$ ter-penetration measurements, it is preferrable to di]ute the upstream concentration of aerosols only and sample the downstream concentration directly. This approach allows a sufficiestly high concentration of aerosols sampled after the filter so that measurements can be made in a reasonabie time. In tegrating the counts over a range of particle sizes also minimizes fluctuations in the calculated filter penetration. However, the range of particle sizes in this integration is generally so broad that the measurenient can no tonger be considered a measurement of monodisperse aerosols. The net effect would be filter-penetration measurements us ing equivalent "quasimonodisperse": aerosols, as in the Lee and Liu's method. Another disadvantage of integrating the particle counts over a broad particle size $r$ ange is that the size range of latex spheres may nat coincide with the size range on the optical counter. This problem is most critical when the peak of the latex distribution falls at the division between adjacent size bins.

It is important to recognize that the problems encountemed in measuring the filter penetration of PSL spheres with aptical particle counters apply to any monodisperse aerosol. Thus, monodisperse aerosols generated by a VOG would also yield large fluctuations in filter penetration. In contrast to the PSL 
spheres, however, the aerosols produced by the VOG do not have a 1 arge concentration of submicron background aerosols, and single-valued detectors, i ike a photometer or electrical aerosol detector, may be used in place of the optical particle counter.

The fundamental problen with filter-test methods based on monodisperse aerosols is the lack of truly monodisperse aerosols between 0.1 and $1.0 \mu \mathrm{m}$ in diameter. The heterodispersion of the PSL aerosols (see Fig. 12) has necessitated the use of an aerosol-yize spectroneter in place of the singlevalued oetector. As previously pointed out, if an optical particle counter is used, then monodisperse aerosols are no longer required. In fact, the monodisperse aerosols greatly magnify the errors made by optical particie counters.

Although it is possible to accomodate the heterodisperse nature of the PSL 1 atex aerosols or the condensation aerosols by accepting a degree af error in filter-penetration measurements, the characteristic low aerosol concentrai:ian and the excessive time requirements make use of monodisperse aerosols impractical for filter testing. We have previausly described how each particle size in the condensation aerosol generator requires a separate solution having a specific concentration of oil. To obtain a filter-penetration curve as a function of partic?e size, each solution has to be connected to the condensation aerosol generator and penetration measurements are made, one at a time. This is extremely time-consuming.

For PSL-spheres, this time-consuming process can te eliminated by placing all of the different latex sizes in a single suspension. An optical counter can then measure the filter penetration for al' of the latex sizes at the same time. Figure 16 shows the particle-size distribution generated from a water

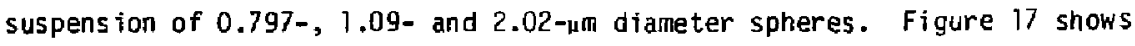
the percent filter penetration derived from the concentration measurements. The large spikes in the penetration curve were caused by the siight shifts in $p$ article size between the upstream and downstream concentration measurements.

Those shifts in particle-size measurements were due to the inability of the logarithmic amplifier. (Nuclear Data, Particle-Sizing Amplifier) to restore the basel ine after processing one pulse. The basel ine offset caused the particle size to shift to smaller values with increasing particle concentration. This type of shift is opposite to the shifts in particle size due to coincidence or non-countable fulses where the shift is toward large sizes with increasing concentration. By replacing the logarithmic amplifier with an 


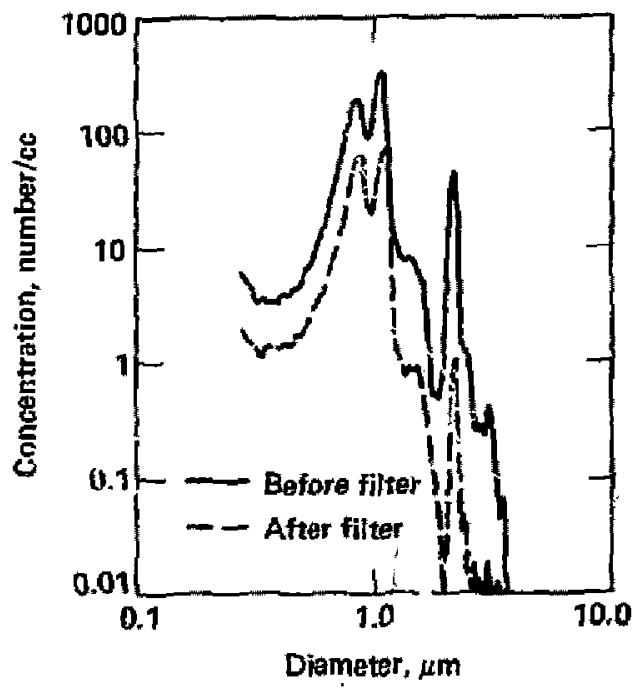

Fig. 16 Particle-size distribution for $0.797 \mu \mathrm{m}, 1.09 \mu \mathrm{m}$ and $2.02 \mu \mathrm{m}$ latex spheres sampled before and after a fiter. Solid curve represents particie-size distribution before fitter and dotted after. 


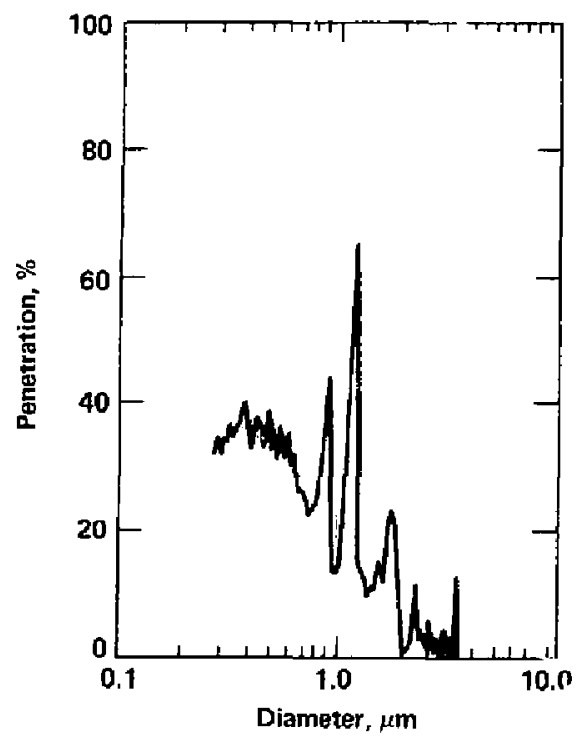

Fig. $17 \mathrm{Filter}$ penetration calculated from the concentration measurements in Fig. 16. The large spikes are due to the small shifts in the measured particle size before and after the filter caused by an electronic baseline shift at higher count rates. 
improved basel ine restarer (MVP Electronics, wideband, DC-Coupled log video Amplifier), we significantly reduced the shift in particle sizes. Figure 18 hows the improved penetration curve for the aerosol containing 0.797-, 1.09 and $2.02-\mathrm{m}$ spheres.

Despite considerable time-saving in simultaneously measuring the concentration of all the monodisperse latexes, figs. 16 through 18 show that the deficiencies in filter testing with monodisperse aerosols are corrected only by making the monodisperse aerosols look like heterodisperse aerosols. It is clear that it is better to use heterodisperse aerosols directly.

The key factors making the use of heterodisperse aerosais more at tractive than monodisperse aerosols are the $h i g h$ aerosol concentration and the fast penetration measurements (Table 2). Aerosol generators are commercially available that can produce high concentrations of heterodisperse solid or liquid aerosols. As previously discussed, a high concentration of aerosols is necessary to provide a sufficient concentration of aerosols downstream of the filter for rapid measurement. A Lask in nozzle is generally used to generate heteroaisperse oil aerosols while a large variety of different nebulizers can be used to generate solid aerosols from a solution. Both of these generators produce a high concentration of aerosols over the particle-size $r$ ange around the maximum filter penetration. By measuring the aerosol size and concentration in one step with a aerosol-size spectrometer, filter penetration can be rapidiy computed from the ratio of the aerosol concentrations at each particle size before and after the filter.

\section{COMPUTER IZED FILTER-TEST FACIL. ITY}

We will break from our discussion of filter-penetration measurements as a function of particle size to describe our unique, computerized filter-test facility. Because of the major advantages of using heterodisperse aerosols and particle-size spectrometers, discussec in the last section, we designed our facility by these two ideas. Three filter-test apparacus were built to support the derelopment of electric air filters: (1) one large-scale test apparatus for evaluating standard ventilation filters $(610 \mathrm{~mm} \times 610 \mathrm{~mm}$ ) at flow rates up to $2,000 \mathrm{cfm}$ and (2) two small-scale test appar.tus for evaluating filter media $203 \mathrm{~mm}$ in diameter at flow rates up to $70 \mathrm{cfm}$. The two smailsc. ?e apparatus were used for fundamental studies (to be discussed in a later 


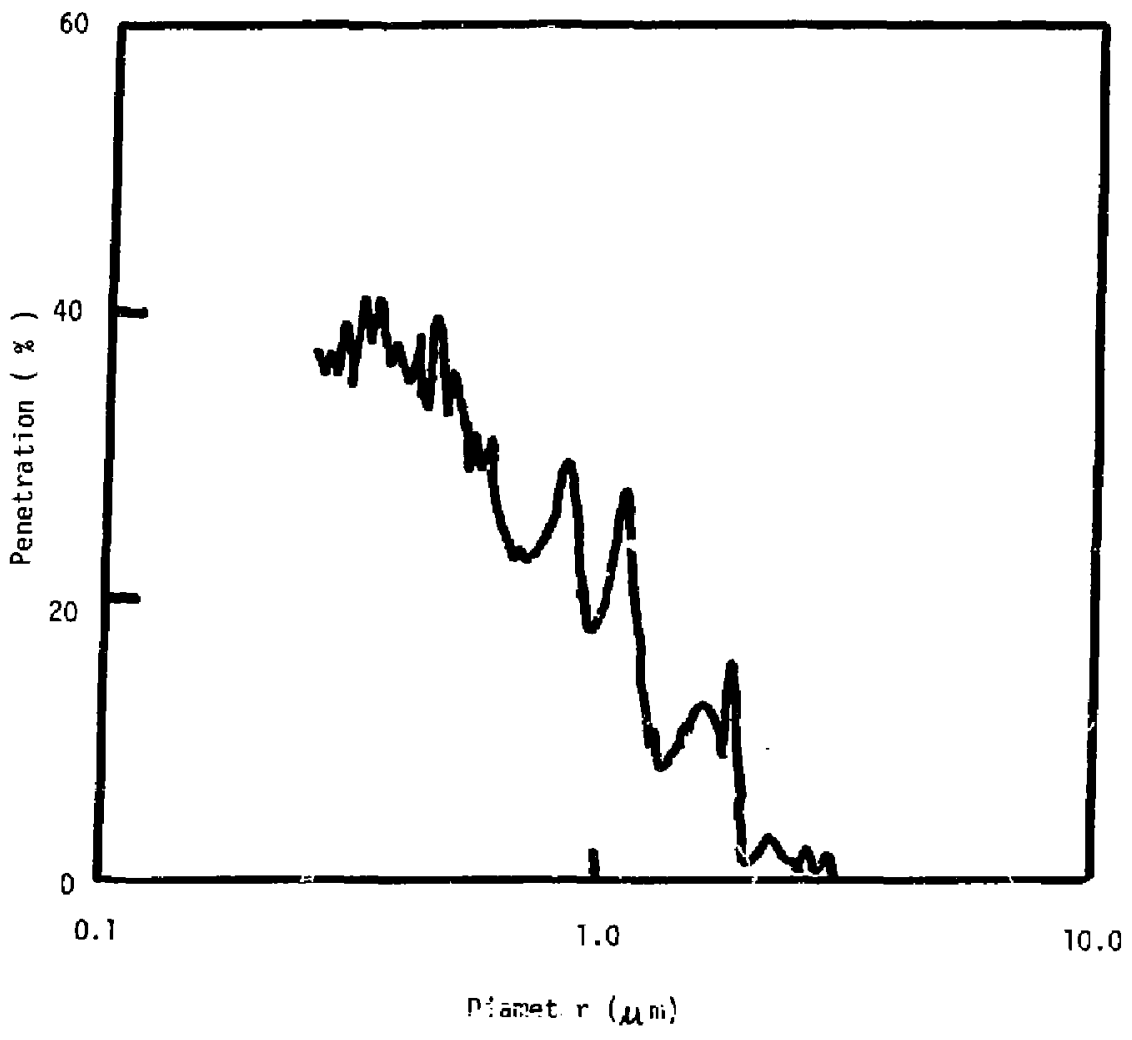

Fig. 18 filter penetration far the same aerosol shown in Fig. 16 after improving the electronics to reduce tne baseline shift at higier count rates. 
section of this report). The large-scale, filter-test apparatus was used for determining the performance of experimental filters under controlled laboratory conditions. All three test apparatus were equipped with heterodisperse aerosol generators and particle-size spectrometers fully interfaced to a $151-11 / 23$ computer to permit rapid filter-penetration measurements as a function af particle size.

We designed the large-scale, filter-test apparatus, shorn in Fig. 19, according to RSHRAE specifications intended for testing air conditioning and ventilation filters for buildings. In place of the recommended dust feeder, we used either Lask in nozzles for generating heterodisperse DES aerosol or Wright nebulizers for generating heterodisperse $\mathrm{NaCl}$ aerosols. We mounted sumple probes before and after the filter housing. Wa also placed a HEPA $f i l t e r$ at the duct entrance to remove atmospheric aerosols. This filter-test apparatus can measure filter penetration as a function of particle diameiar in approximately ten minutes. Larger filter units can also be installed in this filter-test apparasus since the ventilation duct is modular.

A schematic of the two small-scale iilter test apparatus is showa in Fig. 20. The major components of each system are an aerosol generator, a valving system, a test duct and filter assembly, diagnostic instrumentation, and a variable-speed blower. Typically, we atomize a 14 iacl solution to produce a helerodisperse aerosol having an aerodynamic mass median diameter of 0.8 um with a geometric standard deviatio: of 2 .

After being generated, the aerosol passes through a vaiving system where dilution air is introduced or part cf the aerosol is drawn away. Also, the a erosol may be assed inrough charge neutralizers of electrastatic precipitators if a more neutral aerosol is desired. The aerosol then enters an aluminum ouct, passes through the filter undergoing testing. and is exhausted by a variable-speed blower. Flow rates of 14 to $2,550 \mathrm{liters} / \mathrm{min}$ can be obtained in any test, corresponding to velocities at the filter face of 0.83 to $149 \mathrm{~cm} / \mathrm{s}$.

Figures 21 and 22 are photographs of the two smai?-scale, filter-test apparatus. The vertical apparatus in Fig. 22 uses a down-flow design to minimize gravitational settling in the test duct by larger sized particles. Both test apparatus wpre designed to permit rapid insertion of a filter median by separating the central test duct at the sealing flange. Probes mounted before and after the filter were used to bring the aerosol samples to the computer isstrumentation. 


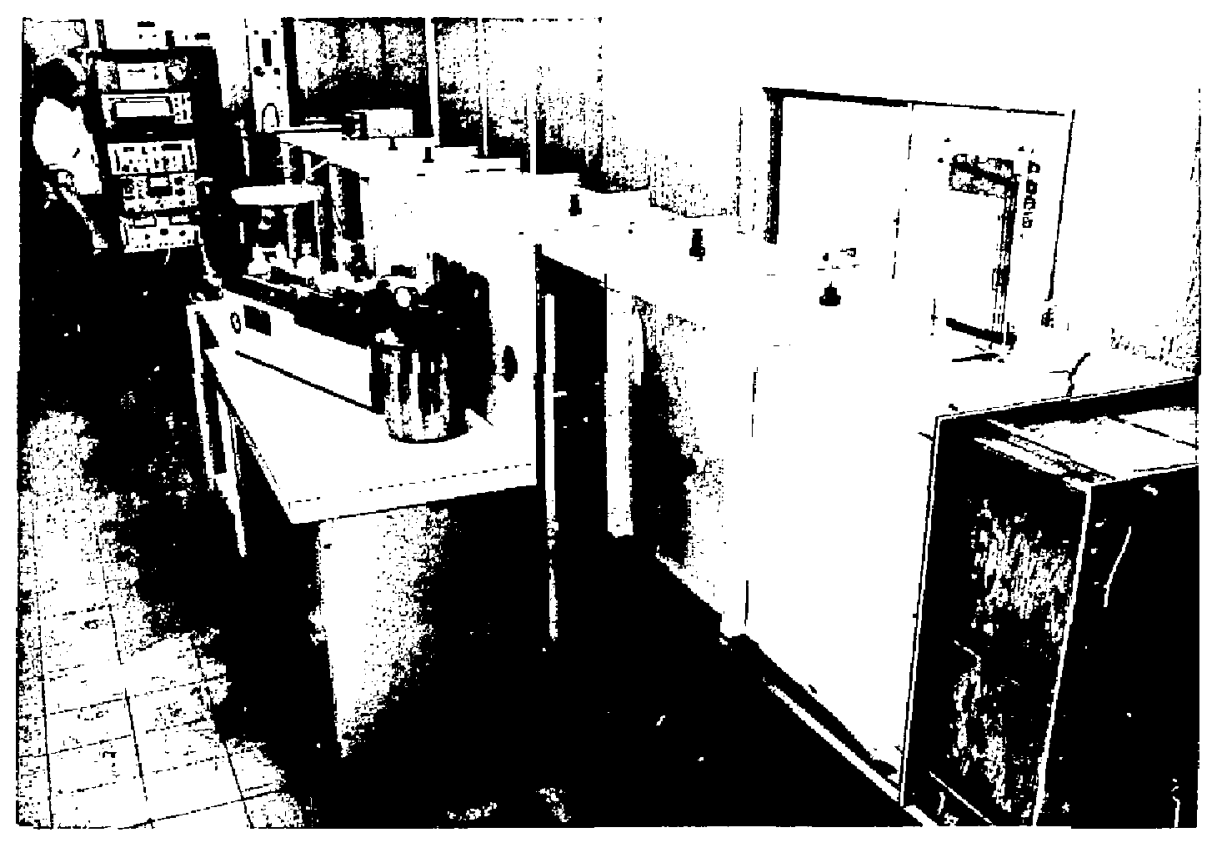

Fig. 19 Photograph of the large-scale filter test apparatus used to evaluate prototype filters up to $2,000 \mathrm{cfm}$. 


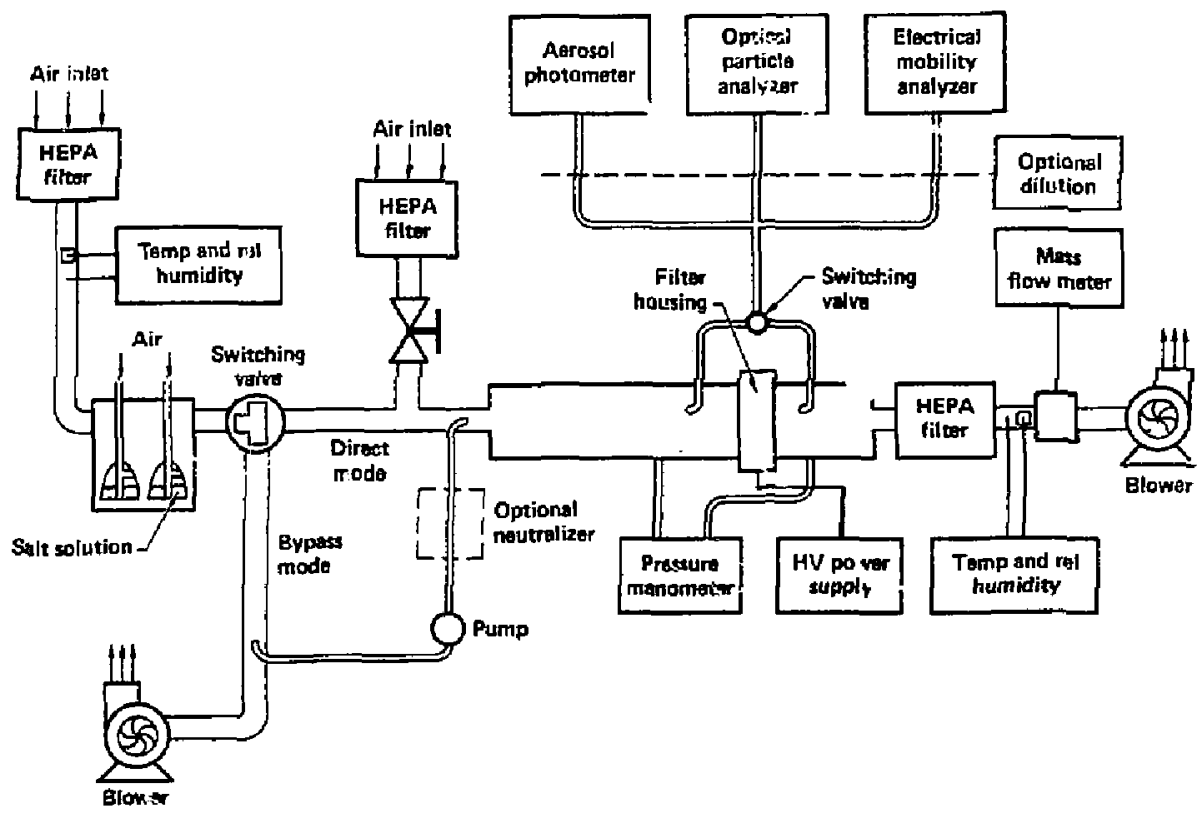

Fig. 20 Schematic of the smali-scale filter test apparatus. 


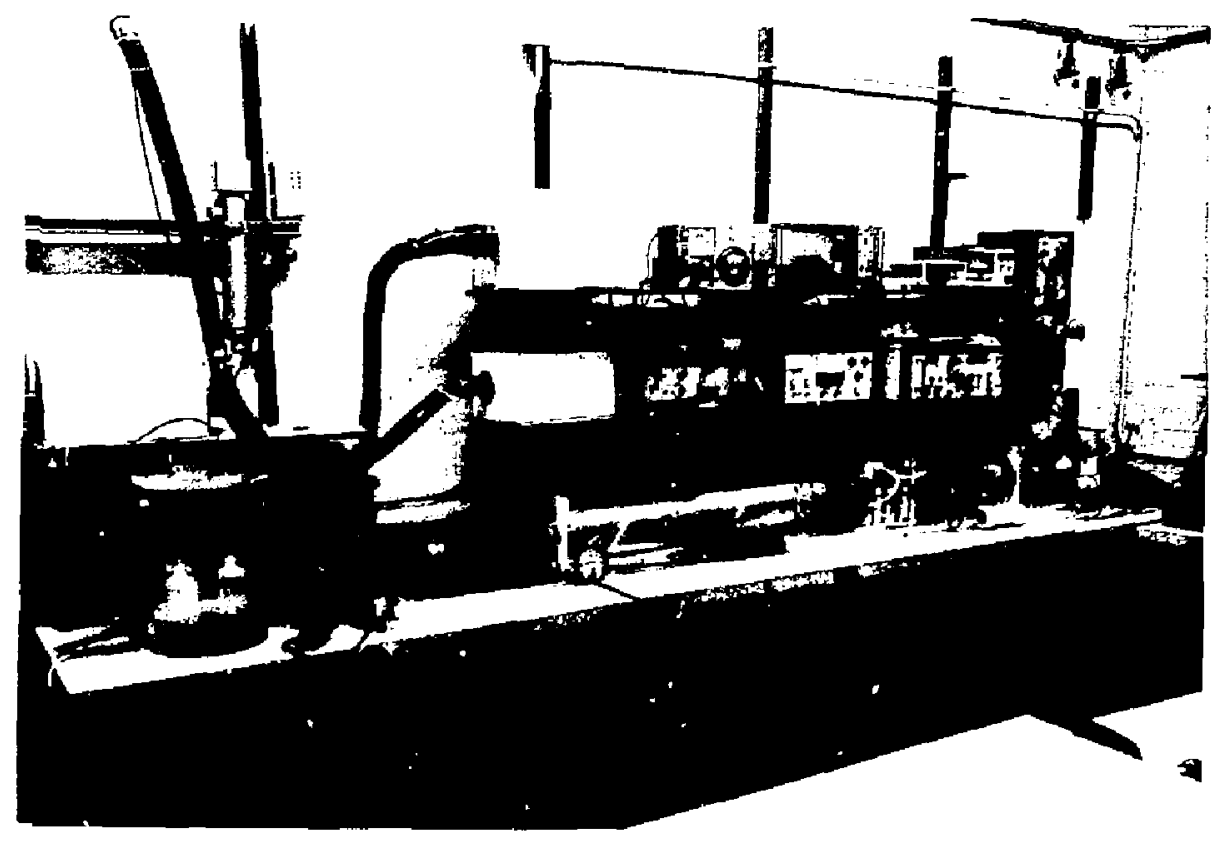

Fig. 21 Photograph of first small-scale filter test apparatus used for fundamental filtration studies. 


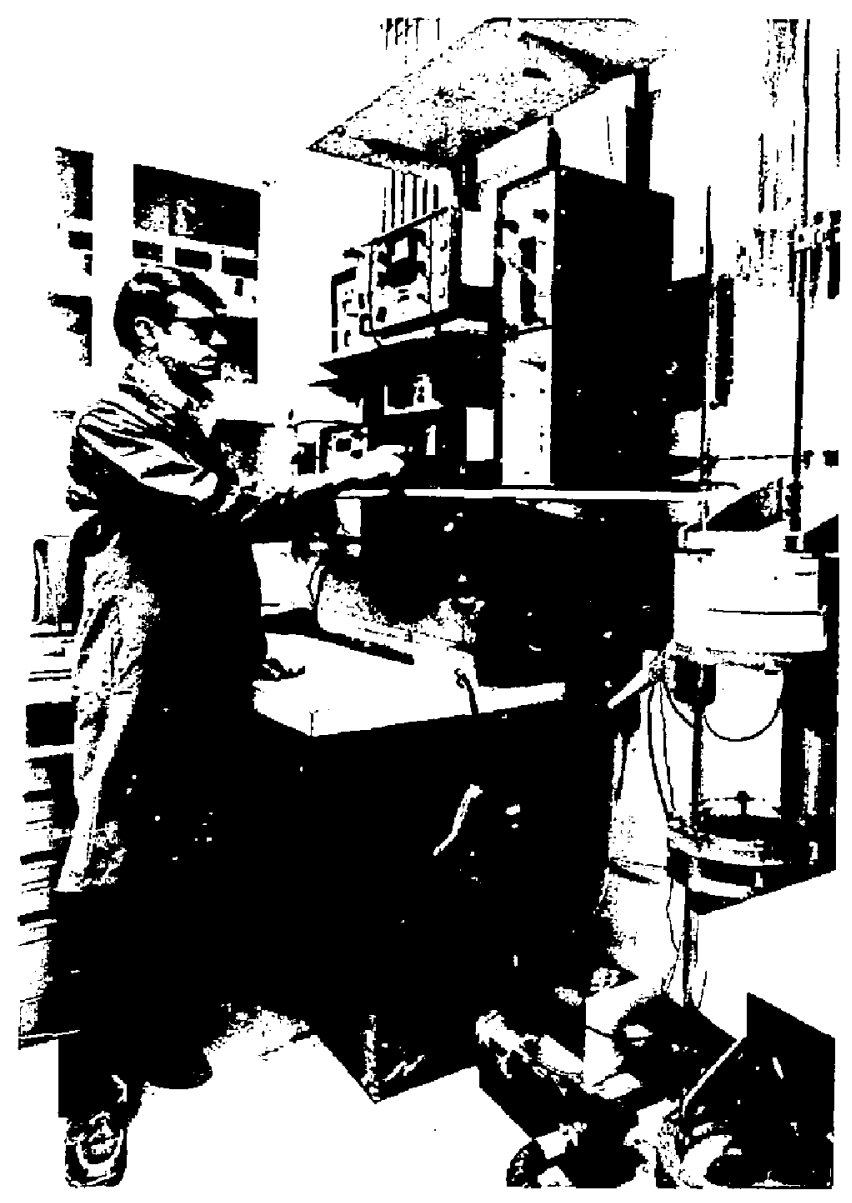

Fig. 22 Photograph of second small-scale filter test apparatus used for funadmental filtration studies. 


\section{FILTER PENETRAT ION TEST INSTRUMENTAT ION}

To measure filter efficiency, we used a Climet-208 particle analyzer, a Thermo Systems, Inc., Model-3030, electrica] aerosol analyzer (EAA), and a Phoenix Precision Instruments, Model JM-7000, aerosol photometer. The Climet partic'e analyzer measures particle size and concentration in the $0.3 \mu$ in to $10 \mathrm{um}$ diameter range by forward light-scattering from single particles. The EAA sizes aerosol particles according to their electric mobility in the $0.01 \mu \mathrm{m}$ to 0.5 um size range. Both of these instruments sample upstream and downstream of the filter undergoing testing so that we can calculate the efficiency as a function of particle size. The photometer produces an integrated 1 ight-scattering intensity from all aerosol particles and quickly indicates overall filter performance. In addition to particle size and concentration measurements, we can measure the totat volumetric flow rate through the filter with a Datametric, Model 770-9628, mas5-flori ueter and the pressure drop across the filter with a Barocel, Model 1174, electronic manometer. Temperature and relative humidity are also monitored.

AI 1 of these instruments are interfaced to an LSI-11,23 computer that has 128K core and uses the Digital Equipment Corporation (DEC) RSX-11M operating system. This system has multi-user and multi-task capabilitits, and it can accept the simultaneous operation of both air-flow systems. The LSI-11/23 is supported by many peripheral devices which include two Western Dynex XL 250-10 hard disks with a combined storage capacity of 20 megabytes anc a 500-byte. Thinker Toy TT80CK-PSC floppy disk. Other peripherals inciude two Tektronix 4025 graphic-display terminals with $32 \mathrm{~K}$ graphic and $32 \mathrm{~K}$ display memory, a Tektronix 4631 hard-copy unit, a Silent 700 keyboard terminai, and a Versatec Model I100AL line printer (Fig. 23).

Initially, we had only three instruments for aerosol masurements interfaced to the LSI 11/23: a Climet optical counter, an $Z A A$, and a 1 ight-scattering photometer. These three instruments were interfaced to the computer in various ways depending upon their type of output. The climet output, consisting of pulses which are proportional to particle size, is sent via a Nuclear Data PSA log Amplifier to a Davidson Model 1056C pulse-height analyzer. Processing the 109 of the scattered light pulses before pulse-height analys is is advantageous because only 128 char.nels are required to store the size distribution from $0.3 \mu \mathrm{m}$ to $10 \mu \mathrm{m}$ dianeter while still maintaining 


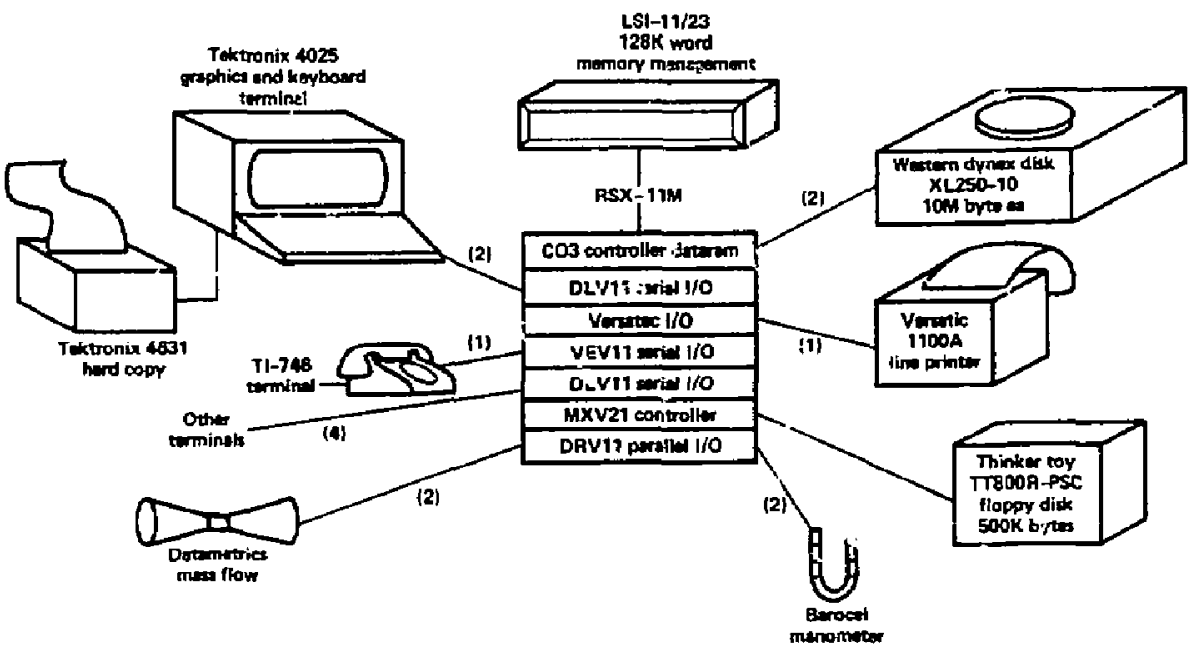

Fig. 23 Schematic showing the LSI-11/23 computer with the major peripheral equipment. 
adequate size resolution. The computer is able to contral the start of pulse accumulation, the stop of accumulation, and the start of data output through the use of a DRVIl parallel I/O card, Data out includes counts in each of 128 channels and is read by the computer via a C'-VII serial I/O card at a baud rate of 9600 . We later modified the Nuclear Data PSA to reduce the baseline shift that resulted in small shifts in particle size, seen in Figs. 13 and 16 and the erroneous fluctuations in filter penetration seen in Figs. 14 and 17.

The EAA data output is in the forth of an analog signal and a pulse indicating the appropriate time to take an electrometer current reading. The analog s ignal is read through an ADAC Corporation Modei-1012 sixteen-channel analog/digital converter. In addition, three control signals are provided: (1)

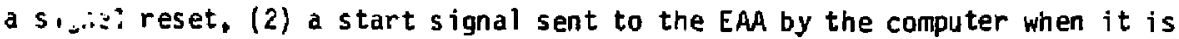
time to initialize the EAA cycle, and (3) a signal from the EAA to the computer incicating the time to read the analog signal. These control signals are handled through the ORVIl parallel 1/0 card.

The third a erosoi instrument, the aerosol photometer, has an analog signal for output. The signal is amplified and sent to the computer via the $A / D$ converter. Mo control signals are required for this device (Fig. 24).

We developed a FOR TRAN program code to allow the experimenter to accumulate, process, sare, and plot size-distribution and filter-penetration data. Besides rapid data analys is and plotting, other key features include reduction of the EAA data by Twoney methods and the combination of the EAA and Climet particle-size distributions in their region of overlap $(0.3-0.5 \mu \mathrm{m})$. Progran output includes prompts to the experimenter, status messages to the experimenter, hard-copy plots, disk-file data storage, and raw and reduced data 1 istings on the 1 ine printer.

An example of the graphic output from a typical measurenent is shown in Figs. 25 and 26 . Figure 25 shows the concentration of $\mathrm{NaCl}$ particles as a function of size for measurements of aerosols sampled before and after the filter. Note that the EAA measures particle concentration only at certain sizes set by the manufacturer, the results of which is the sequence of 1 ine segments snown in Fig. 25 . In contrast, the Climet output has 128 size increments that yield a continuous size distribution. Figure 25 aiso shows that the EAA concentration at $0.5 \mu \mathrm{m}$ diameter is significantly lower than the correspanding concentration measured by the $\mathrm{Climet}$. The problem is due to the EAA's inability to measure particles greater than $0.25 \mu \mathrm{m}$ in diameter. After 


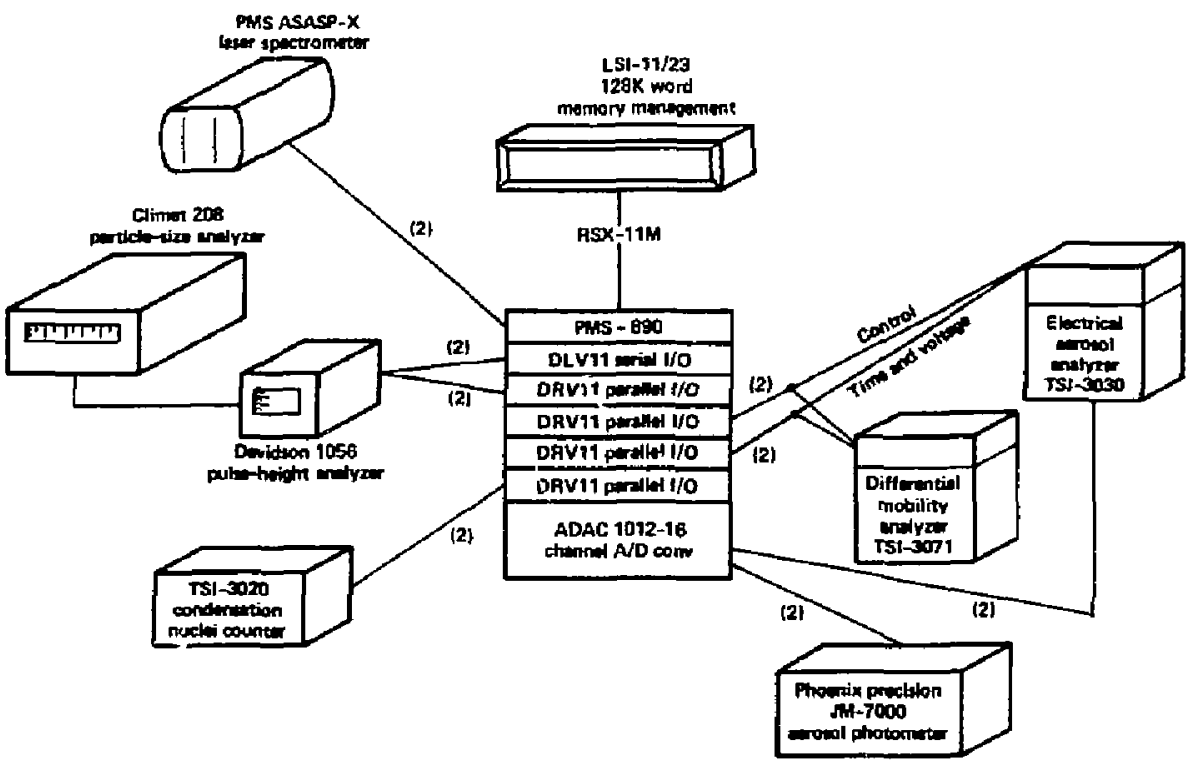

Fig. 24 Schematic showing the aerosol diagnostic instrumentation interfaced to the LSI-11/23 computer. 


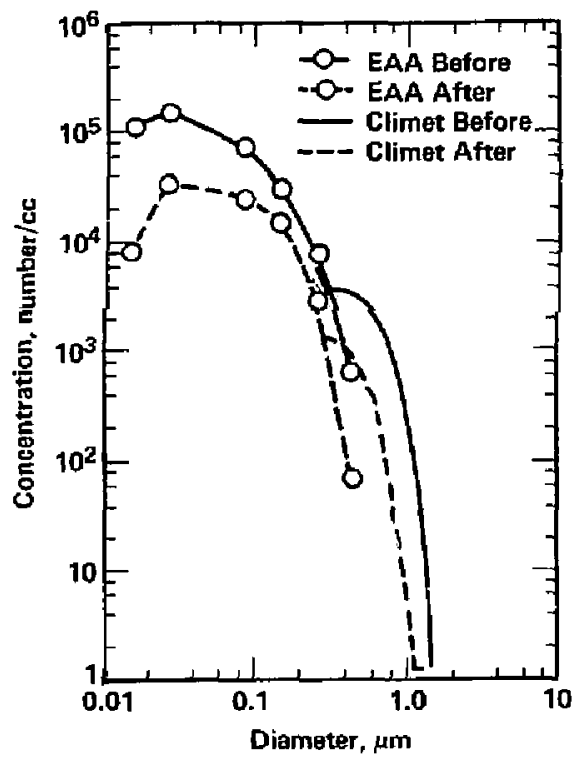

Fig. 25 Typical concentration of NaCl aerosols measured before and after a filter using the EAA and Climet analyzers. 


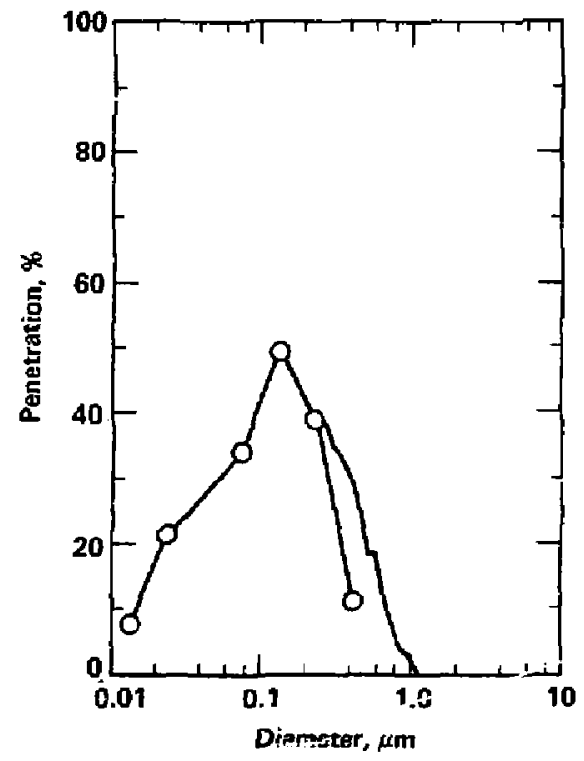

Fig. 26 Typical filter penetration calculated from concentration measurements in Fig. 27. 
the size distribution curves in Fig. 25 are gener:ied, the computer calculates the filter penetration at each particle size by copiputing the ratio of the concentration before and after the filter and gene'ates the graph shown in Fig. 26.

The graphic output can be improved by combining the two curves from the EAA and the Climet using a Spline fitting technique and disregarding the EAA data beyond $0.25 \mu \mathrm{m}$. Figures 27 and 28 show the size distribution curves and the resultant penetration curve we obtained $w i$ th this approach. These curves represent some of the best data generated with EAA and Climet instruments.

\section{Differential Mobility Analyzer and Laser Particle Counter}

The EAA and the Climet had proved satisfactory for most of our tests. However, many tests showed extremely erratic results. The strange results were not caused by improper test procedures since nearly identical results were obtained in repeat tests under carefuily control'ad conditions. We felt the er atic results were due to the EAA and the Climet. Because of the errors inherent in the EAA and the $\mathrm{Cl}$ imet, and the subsequent magnification of these errors, we concluded that the EAA was not well-suited for filter-penetration meas urements .

EAA Errors. Figures 29 and 30 show such an erratic test. The sizedistribution measurenents in Fig. 29 appear to be reasonable, except for the EAA data at the smaller particle sizes. The concentration of aerosols sampled before the filter reaches a maximum and then drops sharply while the coicentration of aerosols sampled after the filter continues to increase. The two curves eventually crass below $0.023 \mu \mathrm{m}$ suggesting a higher concentration after the filter than before. Computing the filter penetration from these curves produced the erratic results seen in Fig. 30 .

After examining possible causes for these results, we concluded that the EAA could not always measure particl? concentrations with sufficient accuracy for filter-penetration measurements, especially at low particle concentrations. Comparing the EAA measurements of aerosols sampled after the filter in Fig. 29 with the corresponding measurements in Figs. 25 and 27 suggested that the primary cause for the bad penetration results was an erroneously high concentration measurement in Fig. 29. An analys is of the EAA operating principle and data reduction showed that the EAA is prone to significant 


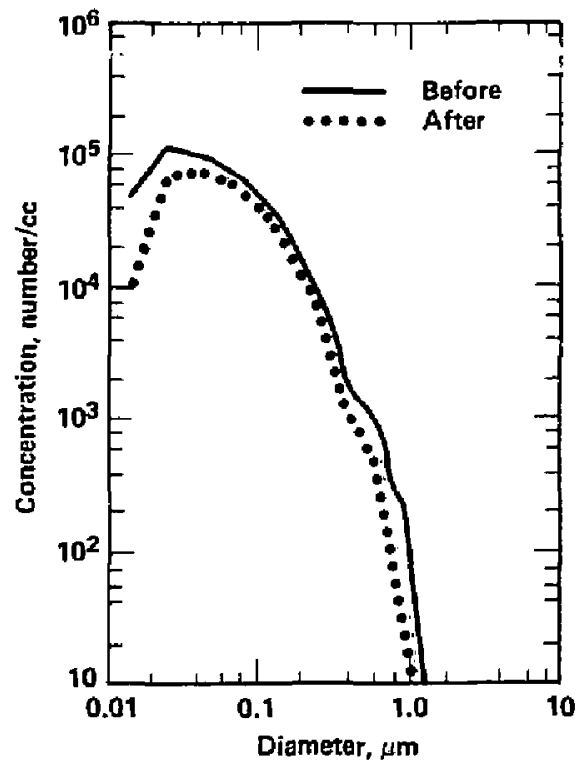

Fig. 27 Improved graphic output showing concentration of $\mathrm{NaCl}$ aerosols before and after a filter using the EAA and $C l$ imet analyzers. 


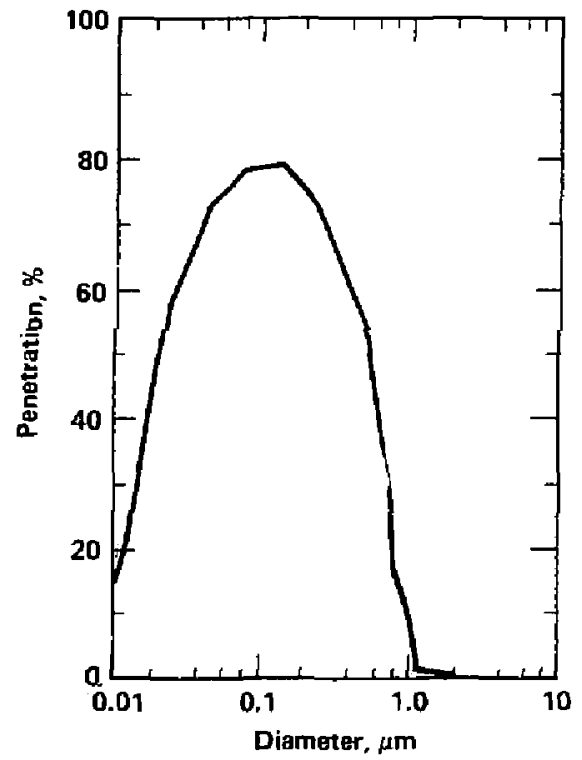

Fig. ¿8 Improvad graphic output showing filter penetration caiculated from the measurements in $\mathrm{Fig} .29$. 


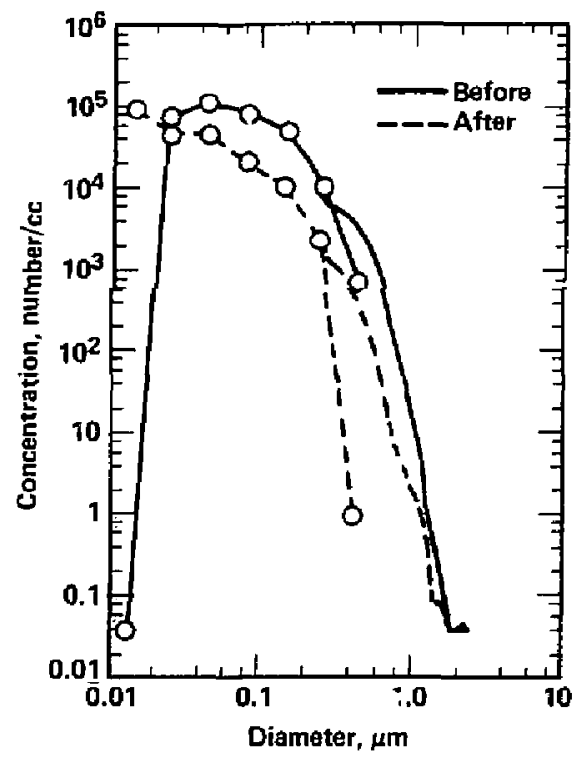

Fig. 29 Typical example of erratic concentration measurements made in a significant number of filter tests. 


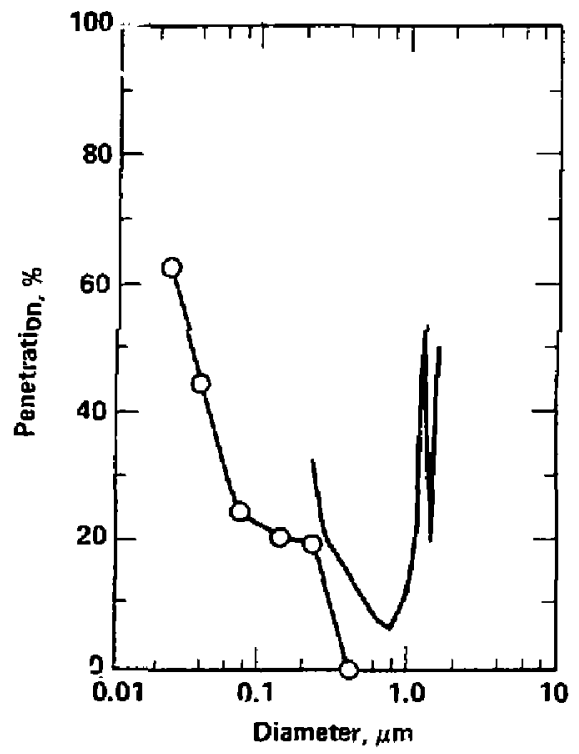

Fig. 30 Filter penetration calculated from the erratic concentration measurement: in Fig. 31 . 
errors in measuring the concentration of particles below 0.1 "Ri in diameter. We should point aut that these errors are significant only in filter-testing. For filter efficiency tes $s$, the concentration at each particle size is important and any error will be reflected directly in the calculated penetration.

The primary source of error in the EAA concentration measurements is due to the difficulty in extracting particle concentrations with in a differentia 1 size $r$ ange from the integrated measurements over a cumulative size range. Figure 31 illustrates the basic components of the EAA particle-size analyzer. A cumulative particle-size distribution is obtained by measuring the concentration of particles exiting the cylinder at each of a sequence of increasing valtage settings until all of the particles are trapped in the cylinder. The EAA detector is an electrometer which measures the current generated by the charged particies captured on a filter. Other detectors, such as condensation nuclei counter, can also be used in place of the electrometer. Positioning the detector at the exit of the mobility-separating cylinder allows us to determine cumulative size distributions.

Particle-size distributions can be derived fr.m cumulative measurements by first taking the difference between the consecutive rieasurements to get a differential measurement that is proportional to the particle concentration within a narrow particle-size range. Particle concentrations could be computed directly from these differential current measurements since the measured current is generated from the sum of the charges on the particles. If each of the particles had a single electron charge, then the number of particles at each size interval could be easily determined by multiplying the differential currents in a constant factor relating current to a number of charges.

Unfartunately, all of the particles do not have a single electron charga. In fact, the number of particles having a single charge decreases rapidly as the particle diameter decreases below $0.1 \mu \mathrm{m}$. Thus, the measurement $0:=$ an extremely small fraction of charged particles is used to determine the total number of particles, the vast majority being uncharged.

Any errors made in measurements are magnified by the multiplication of a large number that compensates for the decreased sen;itivity to smaller particles. If this error is not the same for aerosol measurements before and after the filter, tinen large errors will be made in filter penetration 

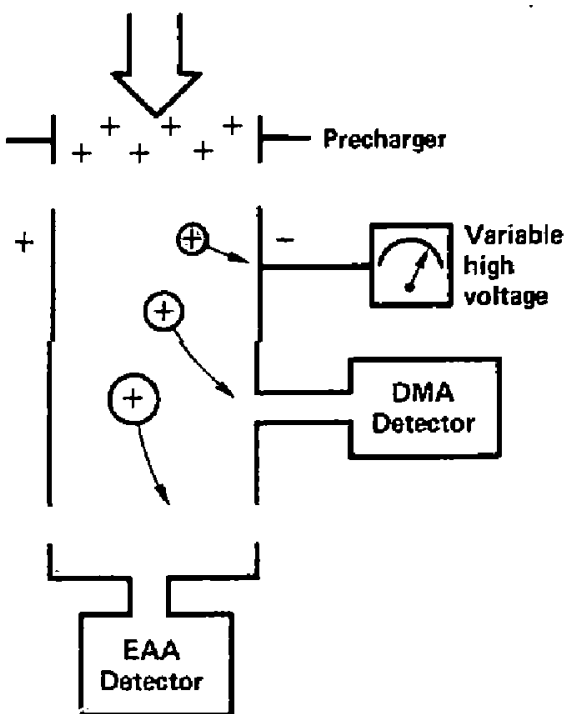

Fig. 31 Schematic showing the basic components of an electrical aerosol analyzer (when the EAA detector is used) and a differential mobility analyzer (when the DMA getector is used). 
measurements. 1t is weli-established that the general approach of deriving differential data from cumlative data will introduce significant errors.

Differential Mobility Analyzer (OMA). Fortunately, the CMA can obtain differential measurements of particle-size distributions directly. Figure 37 compares the essential features of the DMA with the EAA. Common ta both is a precharger to charge the particles and a column to separate the charged particles in an electric field produced by a nigh-voltage source. The major difference between the EAA and the OMA is the positioning of the particle detector. By placing the detector on the colum wali, differential size distributions are measured directly. In this position, only those particles having a proper size will be detected. Smaller particles will be deflected and trapped in the cyl inder before reaching the detector while larger particles pass by.

Al though an electrometer could also be used with the DMA, it is better to use a detecior that can measure the particle concentrations directly. This avoids the problem of decreasing sersitivity to the smaller particle sizes and the use of the large correction factor for sensitivity. An ideal detector for this application is the condensation nuclei counter (CNC), comnercially available from Thermo Systems, Inc. As in all condensation nuclei detectors, the TSI model is based on che growth of small particles in a chamber saturated with a liquid and the subsequent detection by optical means. In contrast to other commercial instruments, the TSI model has the advantage of both photometric detection at high-particle concentrations and single-particle counting at low concentrations. Using a DMA coupled to a CNC allows the direct measurement of particle size distributions without mathematical corrections.

Laser Particle Counter. We also replaced the Clinet optical counter with a LAS- $x$ iaser particie counter obtained from Particle Measuring Systems, Inc. This move was prompted by the inability of the electrical detectors (both EAA and $(M A)$ to accurately measure particle size distributions beyond $0.1 \mu \mathrm{m}$ in diameter. Despite the manufacturer's claims that the electrical detectors can measure particle-size distributions up to at least $0.5 \mathrm{um}$ in dianeter, if not more, we did not find that to be the case. As a result of several hundred tests with the FAA and dozens of tests with the CMA, we concluded that the data was questionable beyond $0.1 \mathrm{ym}$ diameter. This left a gap in our filterpenetration measurenents between 0.7 and $0.3 \mathrm{~km}$ diameter. Since the maximum filter penetration of most filters fall within this region, the gap represented a major deficiency in our filter-penetration measurements. 
Replacing the climet with the laser counter eliminated the gap in our Particle-size measurements. The combination of a laser particle counter and a OMA coupled to a CNC allowed filter-penecration measurements over the size $r$ ange from $0.01 \mu \mathrm{m}$ to $3.0 \mu \mathrm{m}$ withoul: interruptions. These additional instruments were also interfaced to the LSI-11/23. The computer software was modified to use either the EAA in the DMA coupled to a Cilc for the small particle sizes and he r? imet optical counter or the LAS-X laser particle counter for the lar ar particies.

In our prelimilary evaluation of the DMA-CNC-LAS- $X$ instrumentation in filter-penetration tests, we discovered that the penetration curves derived from the DMA-CNC measurements were occasionally erratic (Fig. 32). The prwaie was due to calibration differences in the CNC detector between the photometer mode and the particle-counting mode. In filter tests, the CNC would be in the photometer mode for aerosol measurements made before the filter and in the Particle-counting mode for aerosol measurements made after the filter. If the photometer and particle counter were not calibrated to give the same readings for the same aerosol concentration, we would ob:ain erroneous penetration measurements like those discontinuities in the penetration curve in Fig. 3 ?. Once the two detector modes of the CNC are properly calibrated, a well-behaved penetration curve is obtained. Figure 33 shows a typical filter-penetration measurement using the DMC-CNC-LAS-X instrumentation.

\section{Dilution System for HEPA Filters}

In order to develop an efficient filter test method for HEPA filters, it was necessary to dilute the high concentrations of aerosols before the HEPA filter in order to avoid the coincidence errors and maintenance problems with the laser optical counter (Fig. 34). The degree of dilution is determined by the ratio of the concentration of aerosols in the test duct to the maximum a)lnwed concentration for the particle-size spectrometer. If the derosol Concentration in the filter test duct is comparable to that in the standaro OOP Q-machines, then dilution ratios of approximately 1000 are required. A critical requirement of any aerosol dilution system is that it does not perturb the ariginal particle-size distribution. If this is not possible due to Particle losses in the dilution sytem, then the extent of the losses must be determined so that corrections can be made to the diluted measurements. 


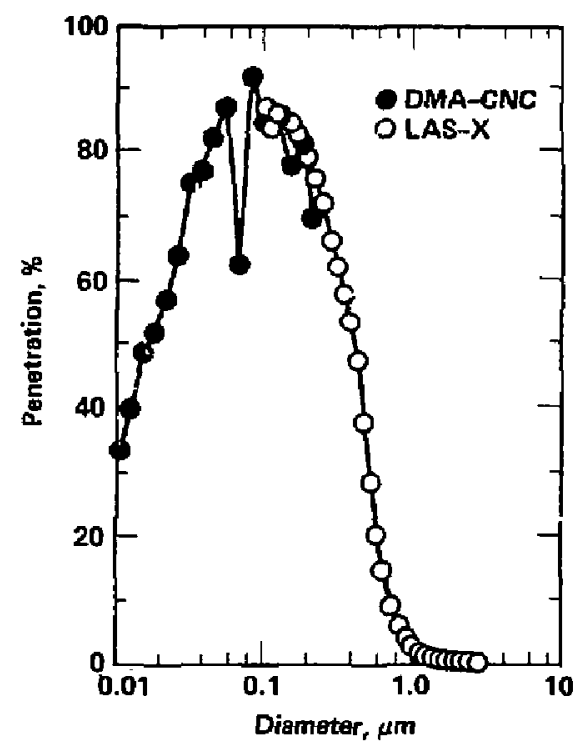

Fig. 32 Filter penetration as a function of particle size determined with the DMA-CNC-LAS- $X$ instrumentation showing the erratic results due to improper Calibration of the CNC. 


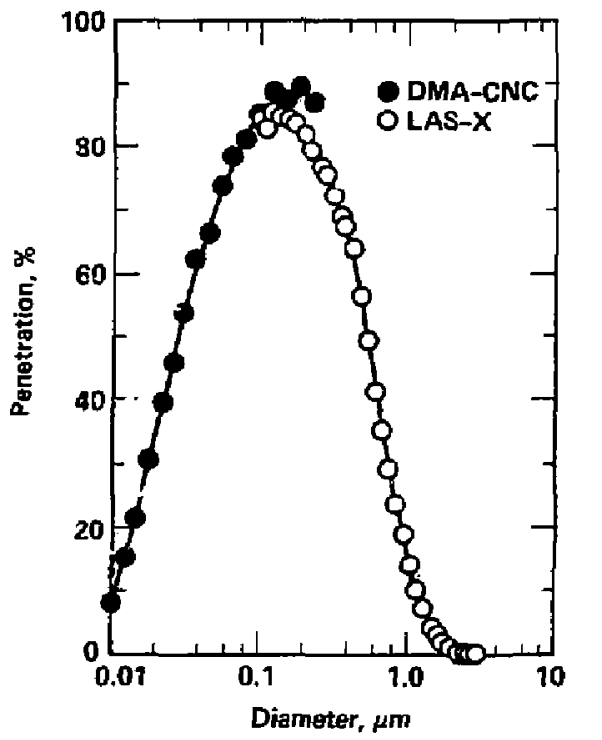

Fig. 33 Filter penetration as a function of particle size after the CNC waS properly calibrated. 


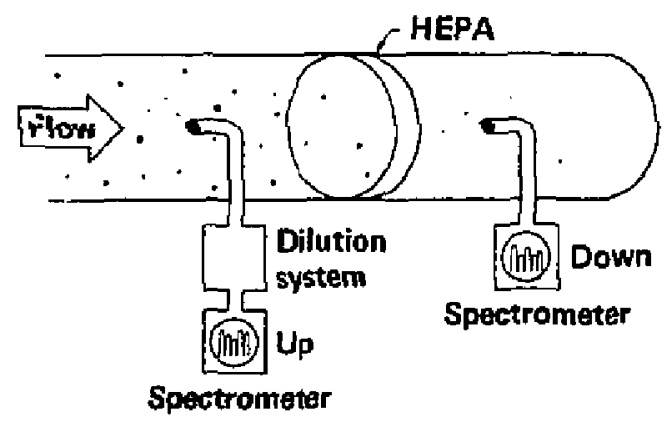

Fig. 34 Schematic of filter test method for measuring filter penetration of HEPA filters as a funticion of particle size by using a dilution system to reduce the upstream concentration of aerosols. 
We evaluated several dilution systems for their use in filter-penetration measurements. One that was the most successful, the $C l$ imet diluter, is shown ir Fig. 35. It was designed to be actached to the inlet of the $\mathrm{Climet}$ optical counter. In this diluter, $0.25 \mathrm{cfm}$ of concentrated aerosol is pulled through the inlet of the diluter and is exhausted at $A$. Filtered dilution air at $0.25 \mathrm{cfm}$ is pulled into $B$ and is exhaustd into the particle analyzer. A needle is used to transfer a si..all volume of the concentrated aerosol to the filtered stream where it is mixed to yield the diluted aerosol. The key operating parameter that controls the dilution ratio is the differential pressure between $A$ and $B$ which determines the flow through the needle. This differential pressure must be carefully monitored since the flow through the needle will be reversed if the vacuum at $A$ is less than at $b$.

We evaluated the Climet di?uter for particle loss using both PSL. spheres and heterodisperse aerosols. Table 3 shows the comparison of the dilution ratios obtained from a ratio of particle counts to the dilution ratios derived from flow measurements. The agreemen $i$ between dilution ratios obtained from particle counts and flow measurements is excellent.

The agreement on comparing the particle counts before and after dilution for heterodisperse $\mathrm{NaCl}$ aerosols is also excellent. Figure 36 shows the computed ailution ratio from these measurements as a function of particle 5 ize is neariy corstant at 100 over the measured particle size range from 0.1 to $3.0 \mu \mathrm{m}$ in dianeter. The dotted lines define the error limits of the measurements which increase at larger particle sizes due to the smaller number of particles. Having a constant dijution ratio over the measured particle size range indicates that few if any particles are lost in the dilution process.

One of the major reasons for this outstanoing performance is the use of laminar flows in the diluter, thereby eliminating particle loss due to turbulent deposition. The $\mathrm{Cl}$ inet diluter was designed for a dilution ratio of 100 and cannot be used for testing HEPA filters. However, by using two Climet viluters in series, we were able to obtain a nominal dilution ratio of 1500 . Figure 37 shows the resulting dilution ratio that was calculated from the ratio of particle counts before and after dilution for heterodisperse Dos aerosuls. Note that the oilution ratio has a parabola shape indicating particle loss for both small and large sizes. Fince these particle losses are constant for a given operating condition, corrections can be made to particle concentration measurements when using the diluters. 


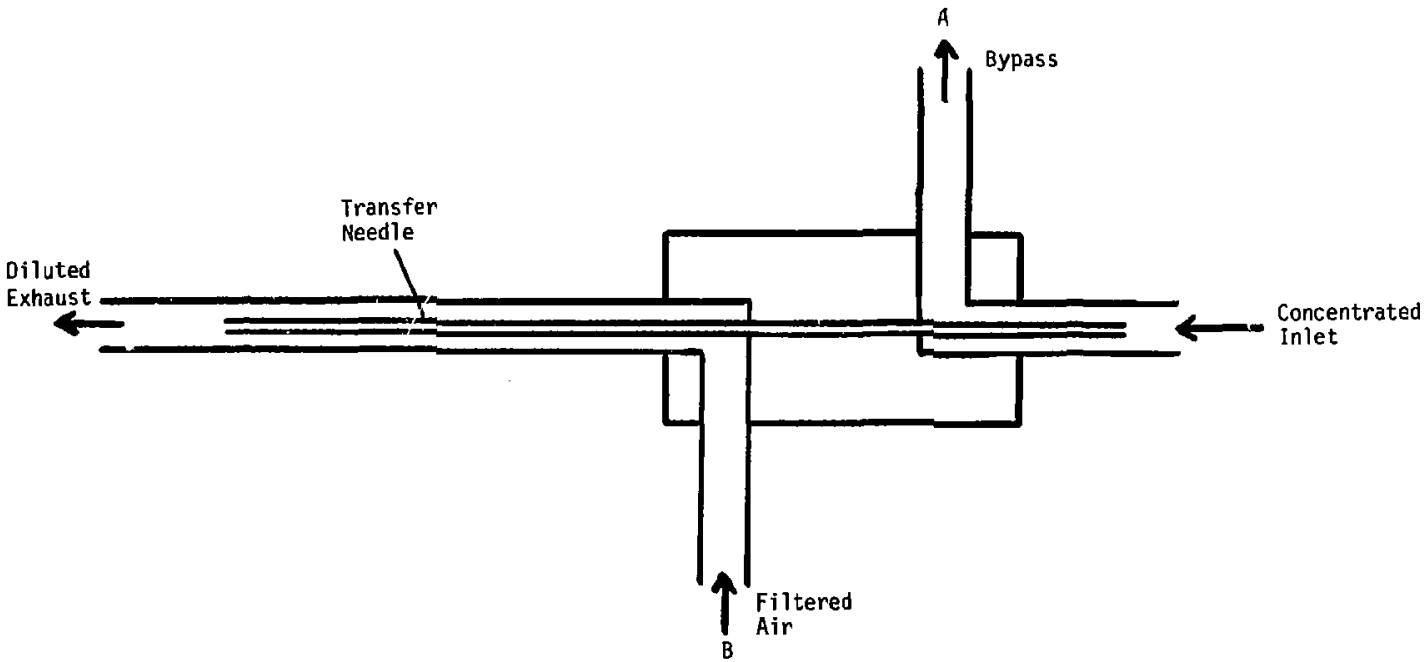

Fig. 35 Schematic of $\mathrm{Cl}$ imet diluter based on the principle of transferring a small volume of concentrated aerosols through a needle to a large volume of

filtered air. The difference in pressure between $A$ and $B$ determines the f:ow through the transfer needle. 
Table 3. Comparison of dilution ratios for $\mathrm{Cl}$ imet diluter from PSL

counts and flow measurements.

PSL Size Dilution Ratio
Count Ratio Flow Ratio

\begin{tabular}{lll}
\hline 2.02 & 37.6 & 37.8 \\
0.8 & 41.2 & 39.8 \\
0.5 & 56.7 & 55.6 \\
2.02 & 37.6 & 37.9 \\
\hline
\end{tabular}

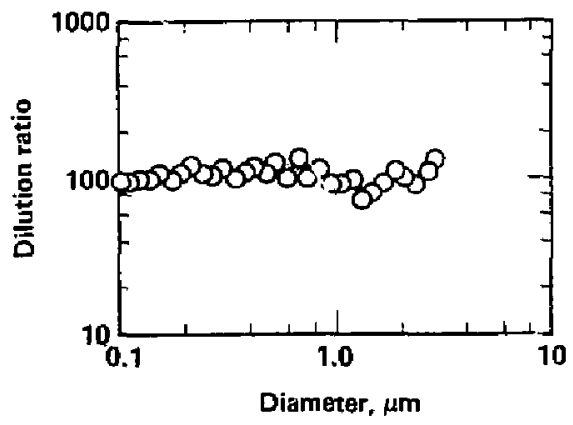

Fig. 36 Dilution -atio as a function of particle size for the climet diluter operated at a dilution ratio of 700 . The relatively constant dilution ratio over the particle side range indicates there is little particle loss. 


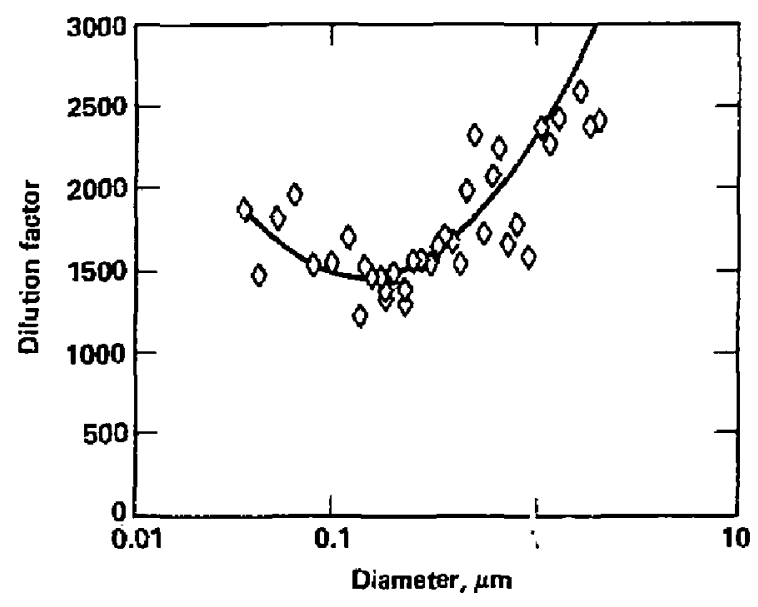

Fig. 37 Dilution ratio as a function of particle size for the climet diluters in series. Solid curve represents least squares fit. 
We used the two $\mathrm{Climet}$ diluters in series to measure the HEPA penetration curve shown in Fig. 8. The particle-size distriution Defore the HEPA filter was measured after ailution while the downstream size distribution was measured directly. Multiplying the diluted upstream concentration by the dilution ratio in Fig. 37 provided the corrected particle size distribution for determining the HEPA penetration curve in Fig. 8 . The HEPA penetration measurement was made in about ten minutes. The development of this dituter was the final obstacle to the completion of our computerized system.

\section{FILTER-EFFICIENCY MEASUREMENTS AS A FUNCTION OF PARTICLE SIZE AND CHARgE}

\section{INTRODUCT ION}

We disccvered during the course of che project, that in the investigation of the filtration process, it is important to measure filter penecration as a function of particle charge as well as size. In this way, the influence of electi ical capture mechanisms can be separated from conventional capture mechanisms. The electronic charge carried by a particle becomes especially important in measurements involving precharged aerosols, polarized aerosols, charged fibers, polarized fibers, or combinations of these. If penetration measurements are made as a function of size only, the capture information of particles carrying different charges is integrated to yield information of particles of the same size. This is analogous to the loss of size information where on ly a photometer is used for penetration measurements.

To better understand the importa.ce of the various electrical capture mechanisms, we developed new instrumentation to measure the concentration of aerosils as a function of both particle size and charge. One iistrument, developed at LLNL, fractionates the particles according to their electric mobility and measures the size distribution of each mobility fraction. A second instrument, still uncler development at the University of Arkansas, simultaneously i.easures the size and charge of individual particles by laser velocimetry techniques. Both methods have the capability of measuring the size and charge of heterodisperse aerosols. We shall discuss these two techniques and present examples of the dependence of penetration upon both particle size and charge. 
Size-charge analysis is accomplished by first fractionating the aerosol according to the electric mobility of the particle. A differential mobility analyzer (DMA), modified to differentiate between positively and negatively charged particles, is operated as a charge spectrometer to extract particles having equal mobilities, or charge-to-size ratios. Secondiy, the particle-size distribution (thiat is, the number of particles in a certain size range) for each extracted mobility is determined using an optical particle counter. By accumusating a size distribution for each mobility fraction, we can determine the aercsol number concentration as a function of size and charge. Because of the inordinate amount of data this process generates, we added a LSI 11/23 computer to control instruments and data, process and store data, and create three-dimensional graphics.

Figure 38 shows the instrumentation configuration, including our modifications. The aerosol is first sampled by a Thermo Systems, Iric. Model-3071 electrostatic classifier; it is a DMA-type, as opposed to the cumulative or first-order :upe, in tha $c$ it extracts particles in a specific electric mobility range determined by electric-field and a ir-flow rates. The aerosol is sampled directly into the DMA colum, bypassing the $85_{\mathrm{Kr}}$ bipolar charger so that the actual charge on the particles remains undisturbed. The instrument is commercially available with a negative high voltage applied to the central roo of the cylindrical column. At a given voltage or electric field, charged particles experience a farce tangential to their direction of flow due to the radially directed electric fielo. Negutively charged particles are repelled vutward to be deposited upon the outer cylinder. Positively charged particles are attracted to the center rod Positively charged particles in a certain mobility range pass through the center-rod aerosol exit, forming the charged-particle sample outlet, $q_{s}$.

Positively charged particles exiting this airstream are analyzed for size and concentration by an optical particle counter. As the voltage is surzessively increased, the size distributions corresponding to lower arid lower constant mobilities are measured until the entire mobility spectrurt of the positively charged particles is determined.

In order to also measure the mobility spectrum of particles carrying negative charges, we incorporated a positive high-voltage power supply and 


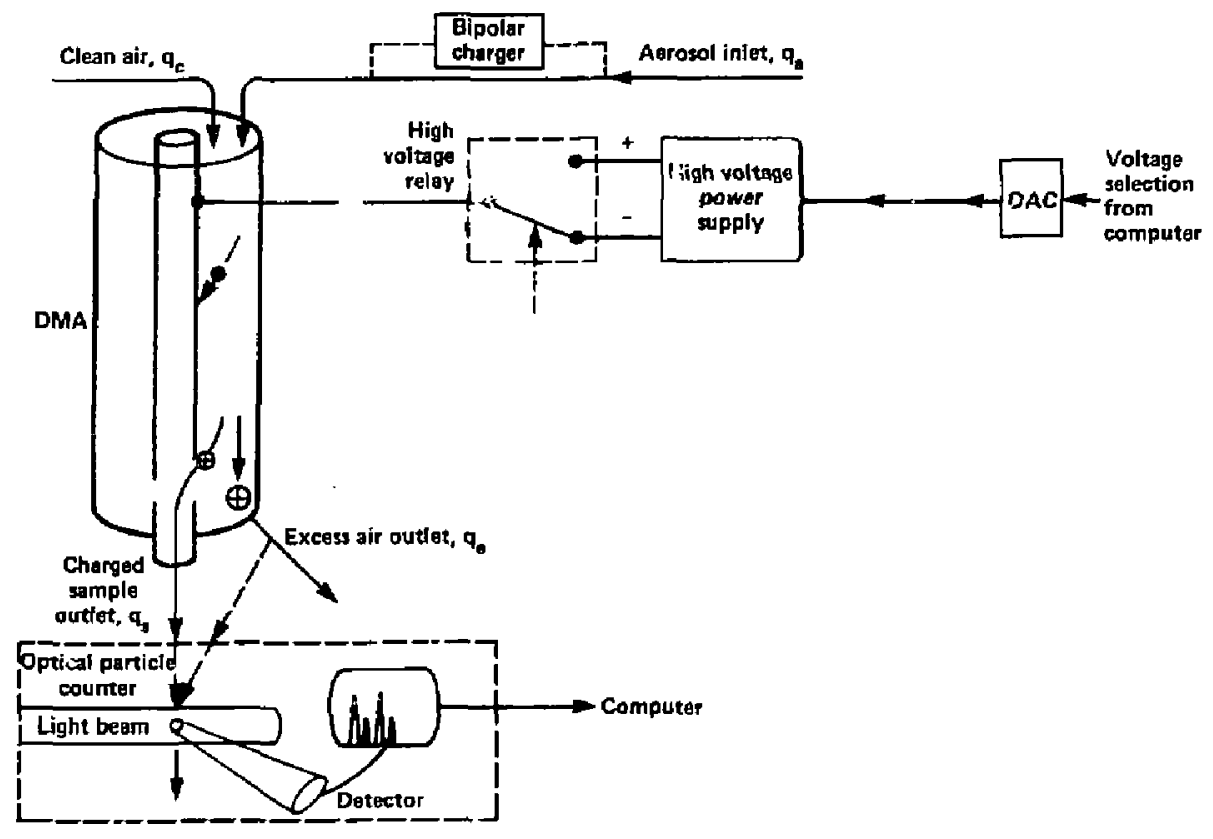

Fig. 38 Instrumentation configuratinn for siz:-charge analysis by mobility fractionation. 
high-voltage relay into the OMA (Fig. 38). When the positive high voltage is applied to the center rod, negatively charged particles, rather than positively charged ones, are collected through the mability-fractionated sample outlet. An inverter amplifier, added to the high voltage regulator, is also switched in when the positive high voltage is chosen.

Far a complete charge-o"stribution anaiysis. it is also necessary to determine the size distribution of particles without charge. For this, we removed the filter in :he DMA excess airstream, and changed the optical counte-'s sampling configuration so that a portion of the excess air was sampled. white in this mode, the greatest voltage is selected in an attempt to remove all charged particles from the excess air outlet. Depending upon the particle size and the air flows through the mobility spectroneter, particles exiting the excess airstream will be neutral or carry only a few charges. Thi,; sample at either the positively or negatively pre-selected $h$ igh voltage determines the "zero" mobility-size spectrum.

We used either a $C 1$ imet-208 particle analyzer or a Particle Measurenent Systems ASASX-P laser spectrometer to determine particle size. With the Climet, the particle-size spectrum, ranging from 0.3 to $10 \mu \mathrm{m}$ in diameter, $C$ an be stored in 128 channels. The laser spectrometer, having a size range of 0.1 to $3.0 \mu \mathrm{m}$ in diameter and 32 channels, has the advantage of detecting a lower particle size than the $C l$ imet. As many as 15 to 60 pre-selected modility intervals might be necessary for a complete charge-distribution measurement. Aad, because a 30- to 50-channel size distribution is accumulated for each mobility intervai, the inordinate amount of data to be processed necessitated the LSI-] 1/23. The experimental apparatus for the size-change measurement by mobility fractionation is shown in Fig. 39.

\section{Mathematical Cerivation of Particle Charge}

We witi now discuss the transformation of the particle mobility to particle charge. The mobility of particles exiting the center-rod sit for a selected voltage, $V_{j}$, for the $i$ th voltage step is given by Liu and Pui ${ }^{12}$ :

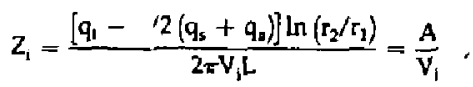




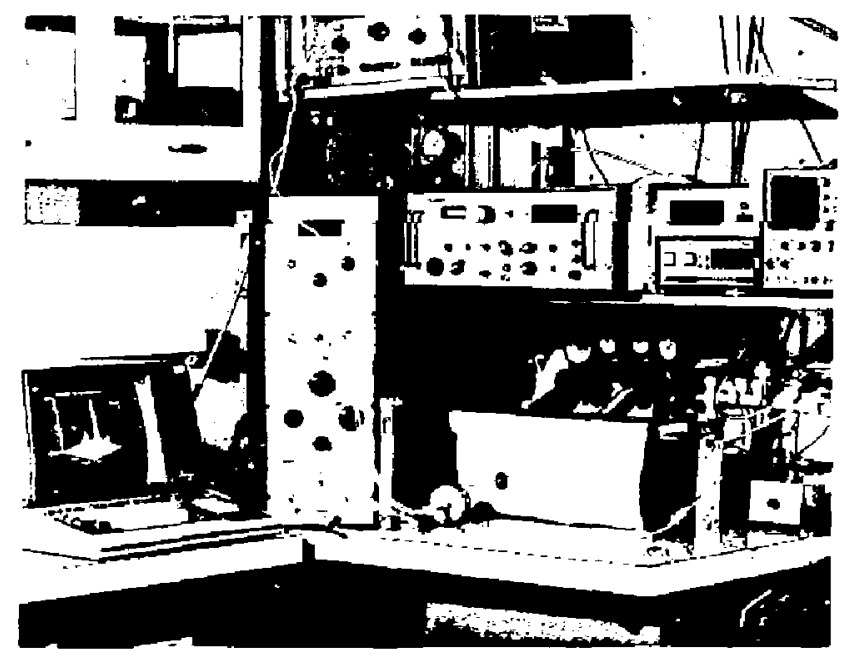

Fig. 39 Experimental apparatus for the sis 2-charge measurement by mobility fractionation. 
where $q_{t}$ is the total air flow $(\mathrm{cc} / \mathrm{sec}), q_{a}$ is the inlet aerosol flow $(\mathrm{cc} / \mathrm{sec}), q_{s}$ is the sample outlet aerosol flow through the slit (cc/sec), L is the distance between the aerosol inlet and the sample exit $(\mathrm{cm}), r_{1}$ is the inner column rodius $(\mathrm{cm})$, and $r_{2}$ is the outer column radius $(\mathrm{cm})$.

Because of the finite width of the inlet and outlet aerosol sample ports, a range of particle mobilities is actually sampled. This mobility range is--

$\Delta Z_{i}=\frac{\left(q_{s}+q_{a}\right) \ln \left(r_{2} / r_{1}\right)}{2 \pi V_{1} L}=\frac{B}{V_{1}}$

The mobility given by Eq. 8 is compared with the electric mobility of a s ingle, charged particle in order to calculate the number of charges carried by a particle exiting the sample outlet flow, $q_{s^{*}}$ The :ingly charged mobiitity is related to the particle size as follows:

$Z 1_{j}=\frac{4.8 \times 10^{-10} C_{i}}{(300)(3 \pi) \eta D_{1}}$

where $a_{j}$ is the particle diameter $(o n), n$ is the air viscosity (P), and $C_{j}$ is the Cunningham slip-correction factor. ${ }^{8}$ Thus, the counts accumulated for each particle size, $D_{j}$, sampied in the mability interval $Z_{i}$ $\Delta Z_{j} / 2$ to $Z_{j}+\Delta Z_{j} / 2$, is assigned the charge value

$Q_{\mathrm{ij}}=Z_{\mathrm{i}} / \mathbf{Z}_{\mathrm{j}}$

Since the single-charged electric mobility depends upon particle size, the upper and i ower isharge limits for a given voltage selection will vary according to size. Examples of charge bounds are illustrated in Fig. 40. When operated at a 15-1 iter/min total flow with a voltage range of 10 to $11400 \mathrm{~V}$, the DMA charge range is approximately 5 to $6000 \mathrm{~V}$ for $1 \mu \mathrm{m}$-diameter particles and only 1 to 250 charge units for $0.1 \mu$ m-diameter particles. A suitable $r$ ange must be chosen 50 the charge distribution lies within these bounds at each particle size of interest.

For later integration of particle counts over charge, it is des irable to have adjacent mobility or charge intervals. This condition can be met for all intervals by using Eqs. 8 and 9 and equating the lower bound of one mosility 


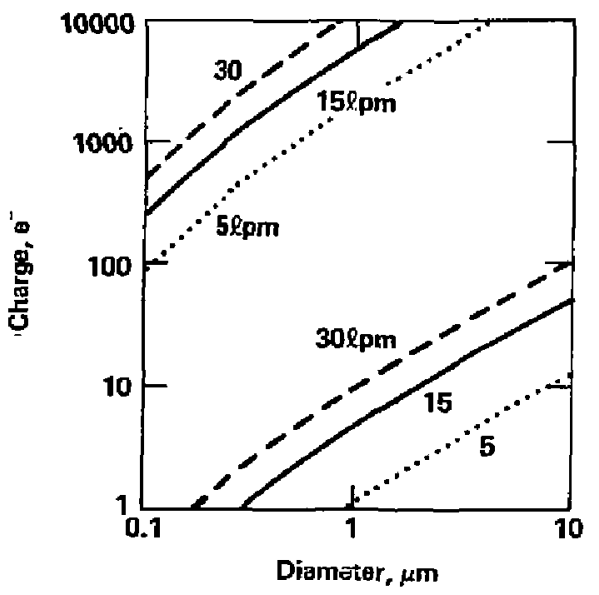

Fig. 40 Differential mobility analyzer charge limits at $10 \mathrm{~V}$ and $1100 \mathrm{~V}$ for $q_{t}=5,15$, and 30 liters $/ m i n$. 
$r$ ange to the upper bound of the next. The resulting voltage recurrance relation for adjacent intervais is--

$V_{i+1}=\frac{(A+B / 2) V_{i}}{(A-B / 2)}$

Correct detection of the number of uncharged particies presented us with a complication. Particles haying charges less than the lower charge limit shown in Fig. 40 passed through the spectroneter and exited $;$ ia the excess air. Such particies were then detected in the "zero" mobility range when the racess air was sampled, and assumed to be uncharged. The problem was alleviated for the 1 arger particles by decreasing the total air flow through the analyzer, thereby precipitating more of the low-mobility, or low-charged particles. Although the "zero" mobility size distrihution then becomes a more exact representation of the uncharged particle distributions, particles having charge between one and the lower charge $I$ imit are not sampled at all. An alternative is to interpolate particle counts between the charge rarge of zero to the lower charge cut-off.

We developed a FORIRAN progran, code that aljowed us to accumulate, process, save, and plot data for the size-charge distributions. rrogram output included prompts to the experimenter, status messages, hard-copy plots, oisk-file data storage, and $r a w$ and reduced data listings. The experimenter is prompted for changes in default values for the DMA flok: rates, optical-counter parameters, and sample time. The program proceeds to set a OMA voltage, wait for a certain equilibrium time period, and then accumulate a size spectrum with the specified optical particle counter. This process is repeated tiitil the entire $r$ ange of pre-selected voltages have been scanned. Equations $8-12$ are then applied to transform the mobility discributions to charge fistributions. We used this tecinnique to evaluate a composite filter using a heterodisperse $\mathrm{NaCl}$ aerosol. The filter, comprised of two layers of Johns-Manville AF-i8 media, was tested at a face velocity of $14 \mathrm{~cm} / \mathrm{s}$. We did not pre-charge the aerosol; charge on the particles occurred as a result of the atonization process during the aerosol generation. The concentration of particles as a furction of size and charge was measured bath upstrean and downstream of the filter. A three-dimensional surface representation of the resulting penetration with size and charge as independent variables is illustrated in 
Fig. 41. The size range of 0.1 to 2.8 ym diameter corresponds to the size range of the laser spectrometer. Grid lines comprising the penetration surface are at equal logarithmic spacing with respect to both particle size and charge. Only the penetration of particles carrying positive charge is included.

Viewing the surface contour from the charge axis, we see that penetration decreases with increasing charge. The spikes on the surface profile occurring in the high-charga regions are due to an insufficient accumulation of counts. In the lower charge region, the penetration has teen equated to zero where no data was accumulated because of the lower charge cut-off limitation of the DMA.

In Fig. 42, we show a collection of penetration curves. Each solid curve corresponds to a different particle charge. The solid curve represents the total penetration as a function of size if only particle size were measured. Each charge curie has the same general shape as the total penetration, having a maximum penetration with diffusion capture dominating at small sizes and impartion at the :arger sizes. However, the size at maximum penetration moves toward larger particle size as the charge increases. For particles carrying higher charge, larger particles are removed more efficiently.

Mobility-fractionation measurements have a wide charge $r$ ange of -1000 to $+1000 \mathrm{e}^{-}$, and a lower size limition of $0.1 \mathrm{um}$ in diameter. Flow settings of the chargc sfectrometer can be adjusted to abtain a charge resolution of $1 e^{-}$if desireo. For a complete size-charge distribution, however, spectrometer flow settings must be chosen to adequately cover the entire ch rige distribution of the aerosol to be measured. Higher particle concentrations than are norma iy possible with an optical counter can be sampled because the mobility-fractionation process precedes detection by the optical counter.

A major disadvantage of mobility fractionation is the long sampling time required ouring which the aerosol concentration and size-charge distribution nust $r$ =main stable. Depending upon the charge resolution desired and the optical-counter sampling period, one complete size-charge distribution measurement may require 30-90 minutes. Thus, the shortast penetration evaluation requires at least 60 minutes. A further disadvantage is that the lower charge cut -off is a function of size, and concentrations below this leve' must be interplated. Often, further interpolations are necessary because many threedimensional surface and contour graphics software packages require equal grid spacings. Investigations of particle losses with in the charge spectrometer, also as a function of size and charge, have yet to be assessed. Further 


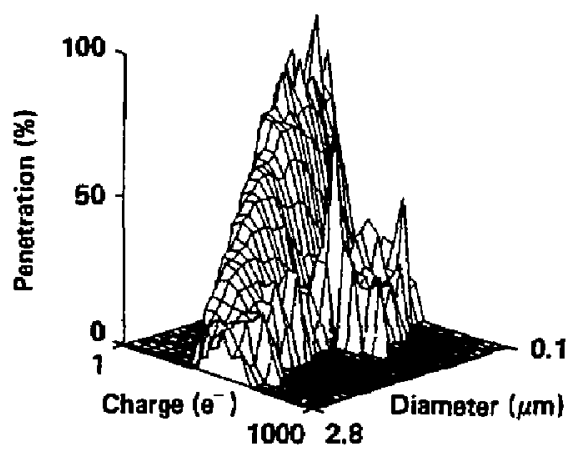

Fig. 41 Penetration surface representation for Manville AF-38 media as a function of particle size and charge.

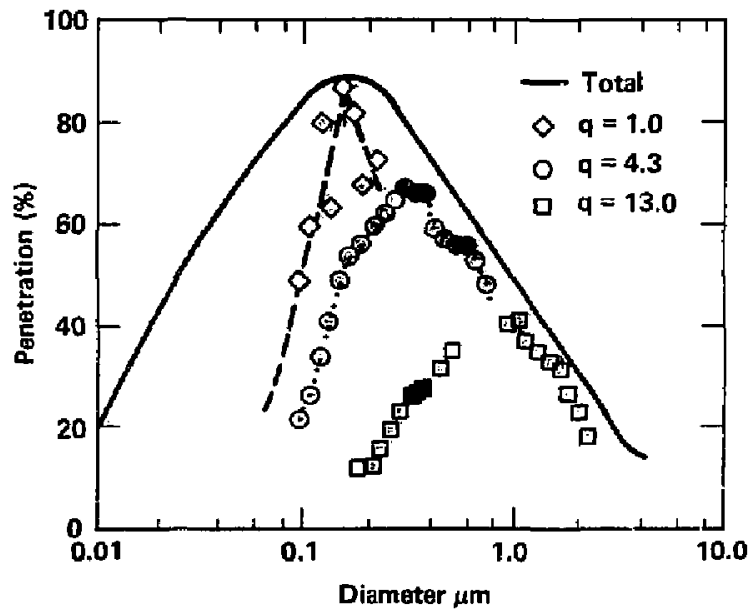

Fig. 42 Penetration as a function of size for cnarge levels ef $1.0,4.3$ and 13.0e-, Manville AF-18 medi:. 
details of the site-charge measurement by mobility fractionation are given by Biermann and Bergman. 13

\section{SIZE-CHARGE MEASUREMENT BY LASER DOPPLER VELOCIMETRY}

Laser Doppler velocimetry (LOV) can measure both the derodynamic size and charge of individual aerosol particles in real-time. The instrument, under development at the University of Arkansas, is called a Single Particle Aerodymanic kelaxation Time (SPART) analyzer. The SPART measures the relaxation $t$ ine of particles after subjecting them to an acoustic (SPART) ${ }^{14}$ or an oscillatory electric field (E-SPART). ${ }^{15}$ The electric field causes particle oscillations whose velocity is medsured by a frequeicy-based differential laser doppler velocimeter. With either the acoustic or elcctric iyeld, the inertia of the particle causes a phase lag with respect to the field frequency. This phase lag is related to the aerodymamic particle size. Operation of the $E$-SPART is not as sensitive to tenperature as the acoustic SPART.

In addition, by measuring the magnitude of oscillation, we can derive particle charge. Unfortunately, uncharged particles, unaffected by the electric field, pass undetected, and are neither counted or sized. A revised E-SPART, currently being developed, uses the acoustic field for excitation and a $D C$ electric field to permit neutral particles to be measured as well as charged particles. In this manner, size information for both charged and uncharge; articles is obtained from the phase lag. while the particle charge is ante ed by measurement of the orift yelacity in the canstant electrit field.

A block schematic of the electronic and optical components of the E-SPART is presented in Fig. 43. The analyzer uses a $15 \mathrm{mw}$ laser $(\lambda=488 \mu \mathrm{m})$. The Lov-sensing volume, approximately $8 \times 10^{-6} \mathrm{~cm}^{3}$, is formed by a 33-degree intersection of two laser beams.

Figure 44 itlustrates the sensing chamber of a modified E-SPAR; having an acoustic field excited $a 25 \mathrm{kHz}$ and a super mposed DC electric field of $2 \times 10^{5} \mathrm{~V} / \mathrm{cm}$. The acoustic driver is electrically grounded while the high voltage is applied on a spherical electrode. As the particle transits the sensitive volume, it experience: an oscillatory motion due to the acoustic field, and if charged, a drift velocity due to the electric f'eld. The particle motion is detected by the LoV. 


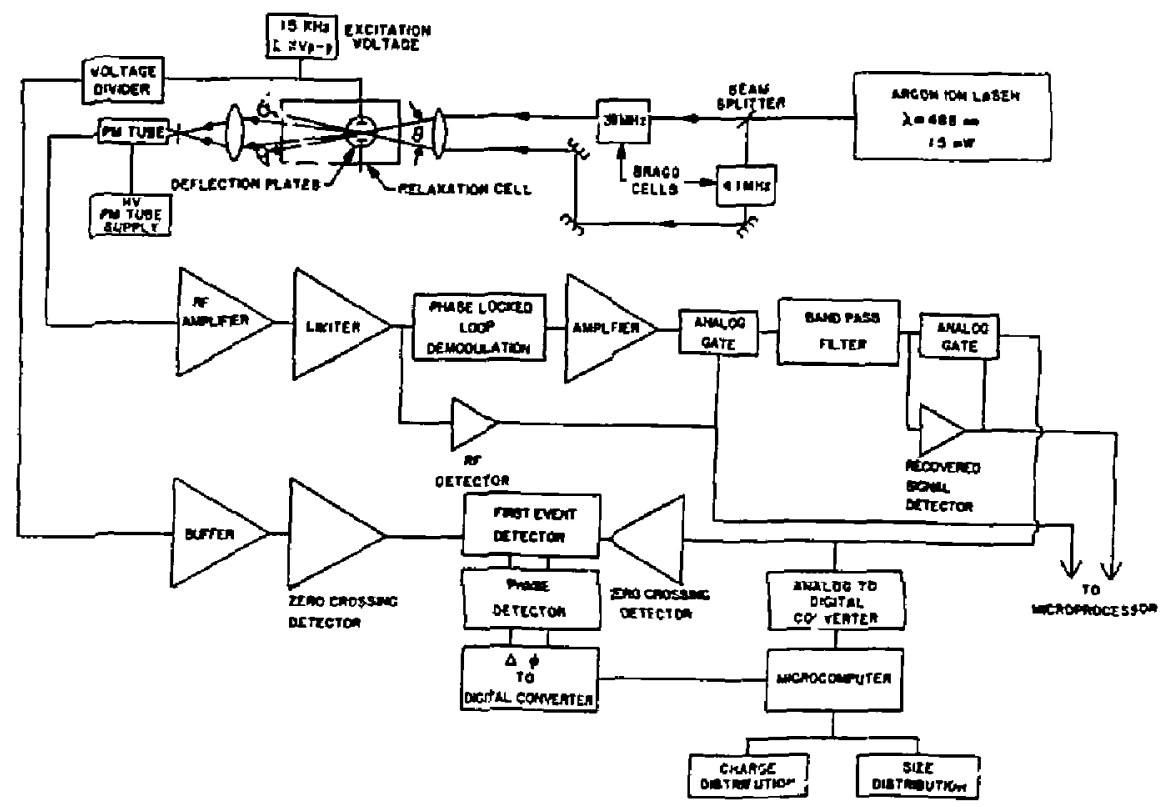

Fig. 43 Electronic and optics block didgram for the E-Spark size-charge analyzer.

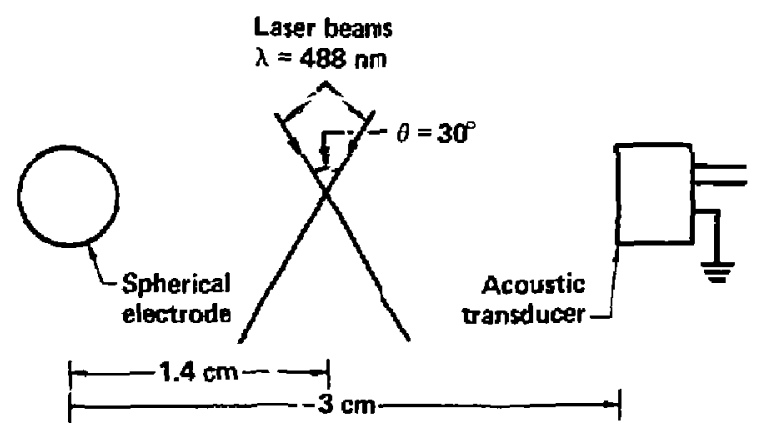

Fig. 44 Sensing chamber for the revised E-SPART, 
The equation for motion of a charged particle in a gas oriven by a uniform acoustic field is

$T_{p}\left(d v_{p} / d t\right)+v_{p}=U \sin (\omega t)$.

where $U$ is the ampititude of the acoustic field, $v_{p}$ is the particle velocity component parallel to the field, and $\omega$ is the angular frequency of the oriving field.

The relaxation time, ${ }^{T} p$, for the particle is given by

$r_{p}=d_{d}^{2} C_{d} / 18 \eta$

where $d_{a}$ is the particle aerodynamic diameter, $C_{a}$ is the Cunningham s i ip correction, and $n$ is the viscosity of the gas. The steady-state solution to the equation of motion, Eq. 13, is

${ }_{F}(t)+\left[U /\left(1+\omega^{2} T_{p}^{2}\right)^{1 / 2}\right] \sin (\omega t-\phi)$.

where the phase 1 lag, $\phi$, is

$\Phi=\tan ^{-1}\left(\omega T_{p}\right)$

Equations 14 and 15 can be solved for the aerodymamic diameter, yielding

$d_{A}=\left[\left(18_{n} \tan \phi / \omega C_{s}\right]^{1 / 2}\right.$

The terminal drift veiocity which a charged particle experiences due to $t$ he eiectric field is

$\mathrm{V}_{\mathrm{E}}=(\mathrm{neCE}) /(3 \pi \eta \mathrm{d})$

where $e$ is the unit of eiectronic charge, $n$ is the number of charges carriec by the particle, $E$ is the electric field strength in the sensitive volume, and 0 is the particle size. The resulting ooppler frequency shift caused by a particie having the velocity is 
$f_{E}=(n e E C K) /\left(6 \pi^{3} \eta d\right)$

where $K$ is the magnitude of the scattering vector of the LDV optical system. Th is Doppler shift, due to scattered radiation, is added to the bias frequency of the LDV, $f_{O}$, to yield a final carrier signal, $f_{S}$, of

$f_{s}=f_{0} \pm f_{E}+f_{d m} \sin \left(2 \pi f_{a} t\right)$

The result is an FM signal whose carrier frequency is $f_{0} \pm f_{E}$ and whose modulating frequency is $f_{a}$, the frequenc: $r$ of the acoustic excitation. Demodulation of this signal results in two outputs: (1) an AC signal which is processed to obtain the phase lag, and (2) a OC signal inc icative of the particle drift velocity in the electric field.

Currently, the modified version of the E-SPART is still being evaluated with regard to particle charge. However, filter-efficiency measurements have been made using monodisperse aerosols and the unmodified E-SPART analyzer. For the results shown in Fig. 45, two layers of AF-18 Mansville filter mesia were challenged with a $0.82 \mu \mathrm{m}$ diameter PSL aerosol. The filter efficiency is shown for both positive and negative charged particles, and also for tile filter under normal operation ( $0 \mathrm{kV}$ ) and electrically enhanced operation (10 kV).

As we have said, a major advantage of the E-SPART analyzer is tife real-time measurement of aerodynamic farticle size and charge of individual particles. The size range of the current instrument is $0.3-3.0 \mu \mathrm{m}$ in diameter. 8ut, it is expected that the lower lir it can be extended to $0.1 \mu \mathrm{m}$ by (1) increasing the laser intensity in the sensitive volume, (2) increasing the solid angle for scattered light detection, nd (3) incorporating a cooled photomaltiplier. The charge range for a l-um-diameter particle is $0- \pm 40$ e- using current electronics and a field strength of $2 \times 10^{5} \mathrm{~V} / \mathrm{cm}$.

Particles having higher charge levels are deflected out of the sensitive volume before being counted. It is estimated that this limit can be increased by * $120 \mathrm{e}-$ with further modifications of the electronics. Particles having higher charge than this would have to be measured by lowering the overall field strength. Since tha slectric mobility is actually being measured, these charge limits vary according to the particle size. Also, the aerodynamic diameter must be converted to a physical diameter before interference from the 


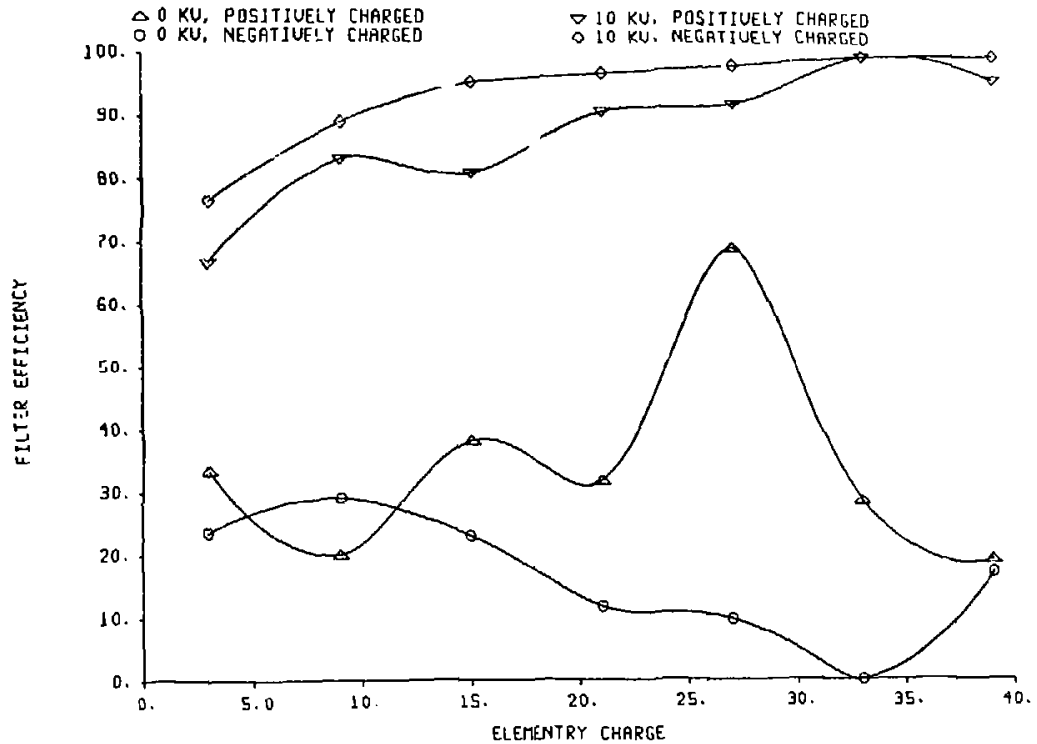

fig. 45 Filter efficiency for positively and negatively charged particles, with and without filier voltage, as measured by the E-spart analyzer. 
electronic charge of the mobility measurement. Although the E-SPART is a real-time instrument, signal-processing time limits actual counting to less than 100 particles/sec. As in the case of size-charge anatysis by mobility fractionation, an appreciable sample time is required for the accumulation of a complete distribution for a heterodisperse aerosol.

FUNDAMENTAL STUDIES OF ELECTRICALLY ENHANCED AIR FILTRATION

INTRODUCTION

A major portion of our electric-air-filter program was devoted to fundanental studies of air filitation, including both mecianical and electrical air filtration. These studies were nezessary in order to lay a foundation for designing experimental filters later on. Although electric air filters had been available commercially in the early $1950 \mathrm{~s}^{16}$, manufacturers had ceased producing them a few years later because of low demand and unreiliable performance, These electric a filters had been introduced into the market without an adequate understanding of their principles and their limitations. Recognizing these previous failures, we have avoided the temptation of designing and building electric filter units in ol- program without first establishing design criteria based on fundamental studies.

A large number of fundamental studies already have laid a good foundation for understanding electric air filtration. However, because air filtration involves a large number of variables and a variety of phenomena, many areas of filtration have been extensively studied while athers have not. Since the objective of our fundamertal studies was to develop a scientific basis for the designing of electric air filters, we used theories that were already established and concentrated on those areas that were poorly understood.

We looked at filtration theory as having four major areas of emphasis or. mechanical and electrical filtration of clean and clogged filters. Mechanical filtratior. in clean filters has been extensively studied and is reasonably well uiderstood. Electrical filtration of clean filters, thouyn, has not been studied t'voroughly and is not well understood. Filter clogging mezhanisms have been investigated even less: mecianical filter clogging is poorly understood while clogging of electric filters is not understood at 611 . 
The objective of our theoretical studies and small-scale experiments was to understand the mechanical and electrical filtration mechanisms for both clean and clagged filters in sufficient detail to optimize the electric air filter. The only reasonably understood filtration mechanisms are those for clean mechanical filters. Therefore, to reach our objective, we has to investigate and develop theories for the othe- filtration mechanisms discussed before. Although the fundamental studies reported here are applicable to a variety of filter media, including granular bed filters, we restricted our investigation to fibrous filters since they are the more widely used.

BACKGROUND OF PROJECT

We selected a design concept for the electric air fi\}ter baseo upon applying an electric field externally across a fibrous filter. This concept was selected over the other approaches for electrification of the filter media because it had the best potential for success in the nuclear industry. The two most important improvements we expected from this electric air filter were (1) improved efficiency and (2) lower pressure drop at comparable mass loadings. He will present evaluations of our externally charged air filter and also several varieties of permanently charged electric air filters. Experimental results relevant to the externally charged filter are compared to a simplified theory we developed that includes filter resistance, collertion efficiency, and transient behavior. The objective of our theoretical studies and small-scale experiments was to understand the mechanical and electrical fitration rlechanisms for both clean and clagged filters in sufficient detail to design experimental electric air fitters. We will also discuss a more rigorous theory, developed by investigacors at the University of Califorria at Davis, that inciudes both mechanical and electrical capture mechanisms.

Electrostatic filters have been investigated since 1930. Althougt thiy can have a number of different configurations, all are based on charging or poiarizing fibers in the filter in order to generate an electric force between the fibers and charged aerocol particles.

The first electrustatic filter was de veloped in 1930 by Hansen, who found that a powdered rerin mixed with wool fibers greatiy improved filter efficiency. 17 itie esin particles that coated the fibers were higilly cherged by the mixing proces. which, in effect, produced charged fiters. This si.jp]e 
electrostatic filter is still widely used today, especially in respirators. Unfortunately, resin filters are ruined when they are exposed to organic vapors which dissolve the resin or to irnizing radiation that neutralizes the resin's charge.

Recognizing the problem of charge dissipation in the resin filter, a num,er of investigators developed methods to continuously recharge the filter by generating charged aerosols upstream of the filter, Rossano a.. I Silverman in 1954 used an electric corona to charge the areosol particles before they entered the filter. ${ }^{18}$ These charged particles were initially trapped in the filter by attractive forces caused by the charged particles until the fibers acquired a high-charge density. Further particle-trapping occurred primarily from coulambic forces between charged fihers and charged particles. Reid and Browne have recently repeated these experiments and have shown high filter efficiencies. 19

Another method for continuously recharging the filter was proposed by Mazumder and Thamas in 1967. 20 Instead of charging the aerosols tc be filte-ed, they injected a charged mist into tine air stream in front of the filter. The charged mist was trapped by the filter fibers which effectively charged them. Oncoming aerosols were then collected by coulombic forces between the aerosols and the charged fibers

Elecirostatic filters have also been made by polarizing fitter fibers externally with an electric field. Although these electrostatic filters have been commercia?ly available since the early 1950s, they have not been widely used. In 1954, Silverman et al., evaluated several compercial electric air filters made of glass fiber mats sandwiched between a high-voltage electrode and a ground screen. 2 ?

Davies wrote an excellent review of electrostatic-filter-appication maiels available up to $1972 .^{1}$ The theoretical investigations showed that the increase in filter efficiency with an applied electric field resulted from the attraction between polarized fibers and charged aerosol parcicles or polarized ftbers and polarized aerosol particles. Rivers gives a good, elementary description of this attraction. 22

The most comprehensive theories were developed by zebe ${ }^{23}$ who ir.urporated the idea of attraction with Lamb's flow field around isoiated fibers. His equations represent the combined filtration mechanisms of interception and electrical forces. A11 recent investigations ${ }^{24-33}$ have 
used Zebel's model to interpret experiments. All of these studies were concerned primarily with the well-established increase in filter efficiency due an externally applied electric field. Although more rigorous theories on electric-field enhancement have been very recently developed by Nielsen and $\mathrm{Hi}_{1} \mathrm{1}^{34}$ and by $\mathrm{Pich}^{35}$, no one has then used to evaluate experimenta? results.

In recent years, studies or electrostatic filters have spread to international groups. Most of these studies were concerned with the parameters that affect filter efficiency due to the electric field. An important new development occurred in 1975 when Frederick ${ }^{3 !}$ reported that a filter also had a significantly lower pressure drop than the same filter without an applied electric field. He postulated that the particle deposits in the electrostatic filter have a more open structure than corresponding particle deposits in the absence of an electric field. Several other investigators have also observed 1 arge reductions in filter-pressure drop with an externaliy applidd electric field. $28,32,36,37$ such results indicated that an electrostatic filter hus a longer service life than a standard filter.

Electric air filters have also been developed that combine particle charging with an externaliy applied electric field. Thomas and Woodfin ${ }^{38}$ fillea the space between plates of an electrostatic precipitator with i glassfiber medium and observed a large efficiency increase compared to the precipitator or to the filter alone. In this configuration, applied high voltage generates an external electric field; it also generates ions in the same region by a corona discharge. Hybrjd electrostatic filiers can also be built by having separate regions for both pariicle-charging and fijtration. Inculet and Castle evaluated a two-stage electrostatic filter having a concentric geometry. ${ }^{39}$ particles charged by a corona dischar'je in a first stage are trapped in a second stage that consists of a filtar medium with a superimposed electric field.

Another kind of electrically enhanced filter is the per nanently charged filter. When we began our program in 1976, the only permanently charged media available was the Hansen filter. Since then, a new generation of permanerriy charged filter media, based on electrets, has been developed by van Turnhout. ${ }^{40,41}$ Electrets, the electrical counterparts of magnets, are formed by heating and cooling a polymer in an electric field. We have investigated several t.'pes of electrets and found that their charge can s dissipated when exposed to certain aerosols and organic vapors. 
Charging Techniques

We selected an ordinary fitrous filter in a constant, external electric field for our studies. Filters where incoming aerosols are precharged by corona discharge were not considered because of the excessive fire hazard. The basic components of the electric air filter described in this report are illustrated in Fig. 46. A fibrous filter medium is sandwiched between two perforated electrodes secarated by a spacer. We chose a wire screen with a $90 \%$ open area for the upstream electrode to minimize the masking of the filter, and we chose a perforated aluminum plate with a $40 \%$ open area for the downstrean electrode. The pressure drop across the two electrodes is typically $5 \mathrm{~Pa}$ at $66 \mathrm{~cm} / \mathrm{s}$. Polyethylene spacers are inserted to maintain the proper spacing for the applied electric field. By applying high voltage to the downstream electrode and grounding the upstream, an electric field generated across the filter medium polarizes the filter fibers. These polarized fibers are responsible for tine increased fitter efficiency.

An important property of this electric air filter is its very low power aquirements. The 200-mm diameter element uses less than $5 \mathrm{~mW}$ of electrical power when $10 \mathrm{kV}$ is applied across the electrodes. Although we used a large, variable OC power supply for laboratory studies, a small power supply is quite sufficient for adequate charging of the filter.

\section{Enhanced Filter Efficiency Experimental Results}

We sumarize here our fundamental-study findings that demonstrate one of the most important: aspects of electric air filters, namely the increastd collection of particles due to electrical interactions. Other experimental tests further describe the performance of the electric air filter; these tests include investigations of the effects of particle size, charge, fiber size and packing density, face velocity, conductivity, transient behavior, and relative humidity. For many of our results, we tried to separate the effacts of these different parameters onfilter performance in order to more easily compare them to theoretical relationships. We conducted these studies in our small-scale filter test facility. 


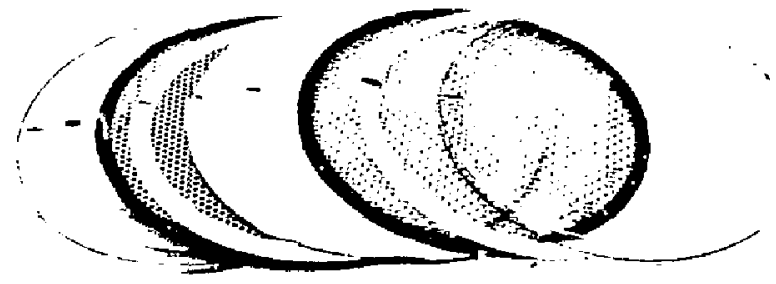

Fig. 46 Disassembled view of an electrir air filter showing the filter media and high-voltage electrodes. 
Mast of our swdies involved two layers of AF-4, AF - 11 or AF-18 media (Johns-Manville, Denver, Colorado) challenged with a NaCi aerosol (un less stated otherwise). The NaCl aerosol, nebulized frem a $1 \%$ NaCl water solution, had an aerodynamic diameter of $1.0 \mu \mathrm{m}$ and a geometric standard deviation of 2.0. Filter properties are list d in Table 4. We often used two layers of a filter media in testing to prevent significant chanriel effects. Fiber-number median diameters and geometric standard deviations, $\sigma_{g}$, were obtained by scanning electron nicroscopy. Fig. 47 shows micrographs for the three types of media.

Particle Size. The enhanced performance of the rilter media when piaced in an electr is field can be iliustraced readily by measuring filter eificiency Is a fur -tion of particle size. "igure 48 shows the efficiency of the AF-18 media at a face velocity of $66 \mathrm{~cm} / \mathrm{s}$. To cover the wide range of particie sizes, simultaneous measurements of the filter efficiency were made using botil a laser particie counter and an electrical mobility analyzer. Figure 56 aiso shows the efficiency of the same filter withnut an electric field and with an applied electric field of $10 \mathrm{kV} / \mathrm{cm}$. The electric field causes a dramatic effect, nearly eliminating the deep minimum in filter efficiency that accurs in conventional filters. This increase in filter afficiency occurs with no corresponding increase in pressure loss.

Although the elimination of the deep minimum has not been a recognized attribute of the electric a: - filter, this experimental result can be easily predicted. Since very large and very smali particles are captures effectively by a conventionai fibrous filtisr, the particies that are able to penetrate the filter must fali within these toundaries. It has also been well establishe $J$ that applying an electric fiel.J to a filter greatly increases the filter efficiency. He can conclude, combining these two facts, that the electric field reauces the particle penetration in the size region characterized by a mintmum fi?ter efficiency. Using this fiber media with this flow rate, the minimum occurs in the submicron range.

The efficiency data in Fig. 48 were determined for particles haying a known size distribution but an unknown specific charge distribution. It is highly probable the particles in each size range had a distribution of charges centered about zero, with the large particle sizes having a broader charge distribution. Aithough nur size-charge aerosol instruatentation for decoupling particle size and charge effects was not fuily developed at this time, by measuring the efficiency as a function of particle size, we were able to see 
Table 4. Properties of Manville AF-4, AF-11, and AF-18 filter media.

\begin{tabular}{|c|c|c|c|}
\hline $\begin{array}{l}\text { Media } \\
\text { Type }\end{array}$ & $\begin{array}{l}\text { Packing } \\
\text { Fraction }\end{array}$ & $\begin{array}{l}\text { Fiber } \\
\text { Diam ( m })\end{array}$ & $\begin{array}{l}\text { Pressure } \\
\text { Loss (Pa) }\end{array}$ \\
\hline$A F-4$ & 0.0028 & 0.9 & 110 \\
\hline$A F-11$ & 0.0038 & 2.6 & 21 \\
\hline AF -18 & 0.0086 & 3.9 & 13 \\
\hline
\end{tabular}




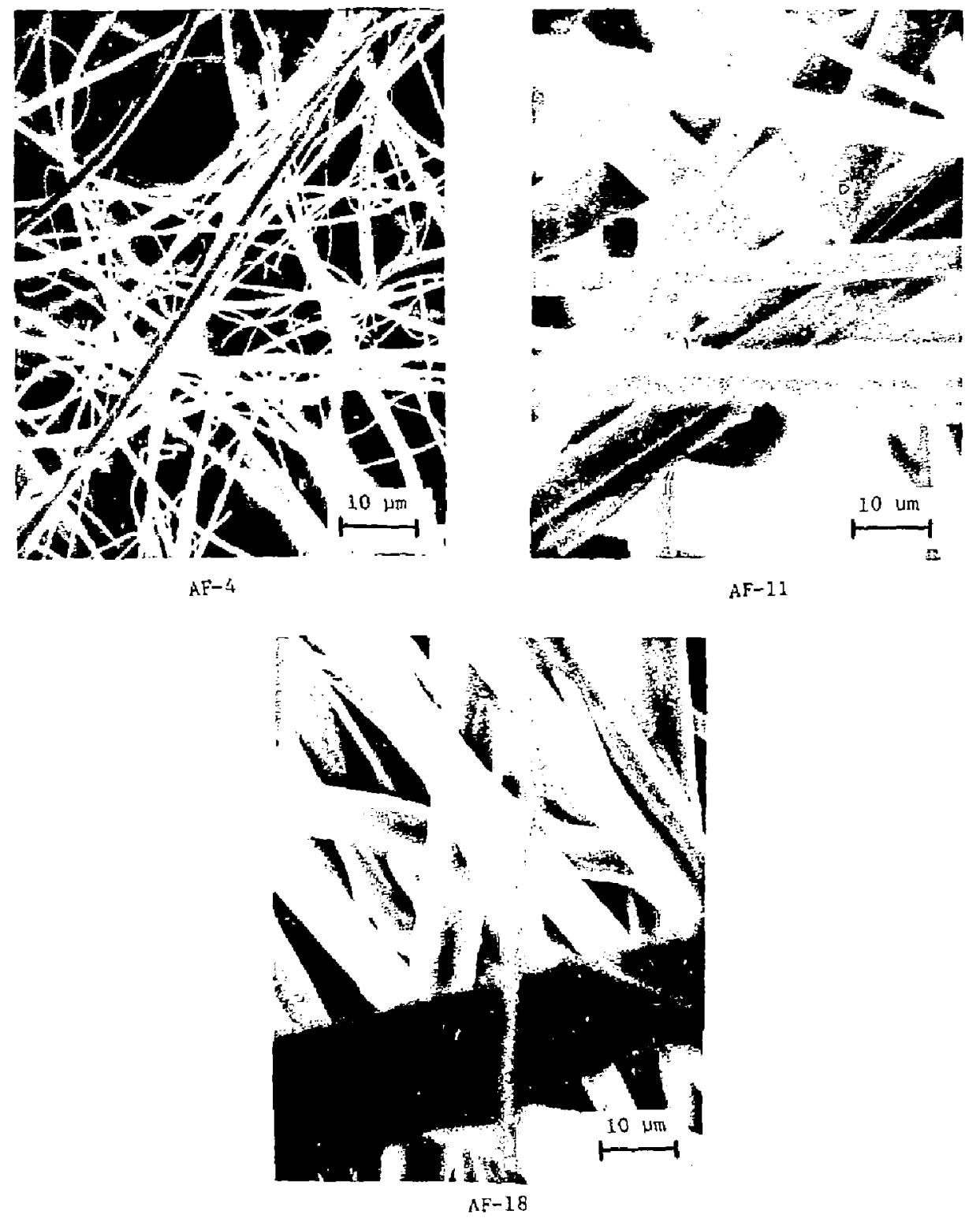

Fig. 47 Electron micrographs of AF-4, AF-11 and AF-18 filter media. 


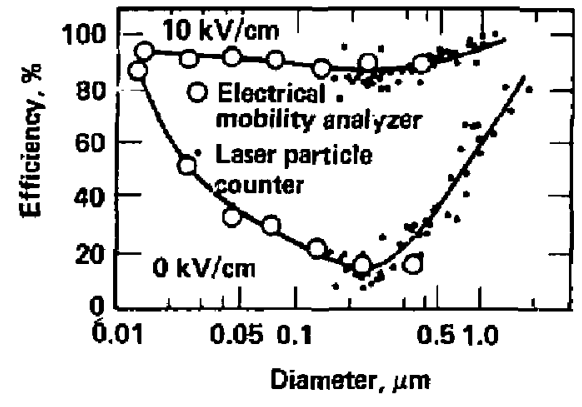

Fig. 48 Filter efficiency as a function of particle size with and without an e iectric field using electrically charged aerosols. 
changes in the filter efficiency with changes in the averge particle charge. Figure 49 shows the results of a filter efficiency test conducted under the same conditions used in $F i g .48$ except that the particle charge was reduced from 13 to 2.5 units using a $85_{K r}$ source.

A comparison of the curves in Figs. 48 and 49 shows that reducing the average particle charge from 13 to 2.5 units had no effect on the filter's effiziency without an electric field. However, the same reduction in particle charge shows a significant reduction in the efficioncy when $10 \mathrm{kV} / \mathrm{cm}$ was applied across the filter for particles ranging from 0.02 to $0.2 \mathrm{um}$ dianeter. Above 0.4 um in dianeter, the reduction in particle charge had no effect on filter efficiency.

If we assume particle charge was reduced proportionally for a? 1 sizes, then the efficiencies for the electric air filters in Figs. 48 and 49 have a simple, qualitative explanation. For particles above $0.4 \mathrm{\mu m}$ in diameter, the electric air filter is dominated by a polarized-particle, polarized-fiber mechanism and shows no dependence upon particle charge. Between 0.02 and $0.2 \mu \mathrm{m}$ in diameter, a charged-particle, polarized-fiber mechanism plays a major role in filter efficiency and ts strongly dependent upon particle charge. A1though this simple explanation accounts for our experimental results, intil particle-charge effects are decoupled from the particle-size effects, we must leave it as speculation only.

Face Velocity. In Fig. 50(a), filter efficiency determined by a flame photometer is illustrated as a function of the applied electric field. The same filter was tested at face velocities of $0.65,0.32$, and $0.76 \mathrm{~m} / \mathrm{s}$. Without an electric field, the filter efficiency increased as the flow velocity increased, while the opposite occured at higher electric-fjeld strengths.

without an electric field, filter efficiency is determined by only mechanical capture mechanisms. Since the flame photometer measures a quantity proportional to particle mass, the efficiency measurement is primarily a measurement of the inertial capture mechanism. Thus, at higher velocities, the particle inertia increases, thereby increasing the efficiency. When the electric field is applied, the primary factor controlling the efficiency increase is the residence time of the particles within the filter medium. The longer the particles remain within the filter medium, the greater time the electrical forces have to attract these particles to the fibers. 


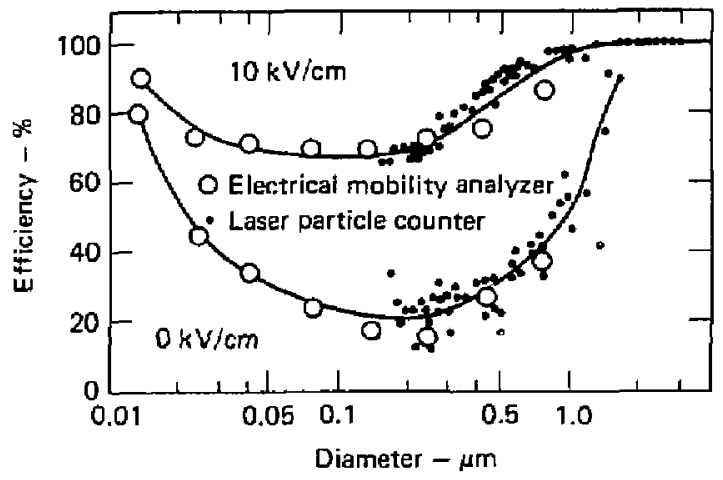

Fig. 49 Filter efficiency as a function of particle size with and without an electric field using neutralized aerosols. 


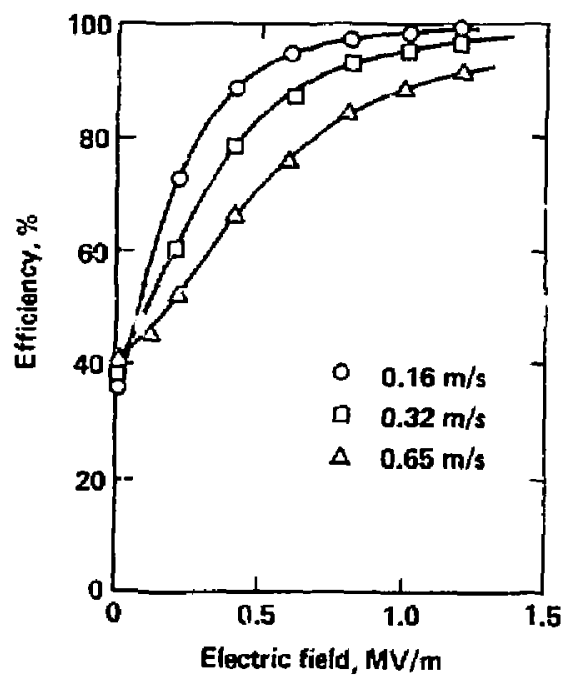

(a)

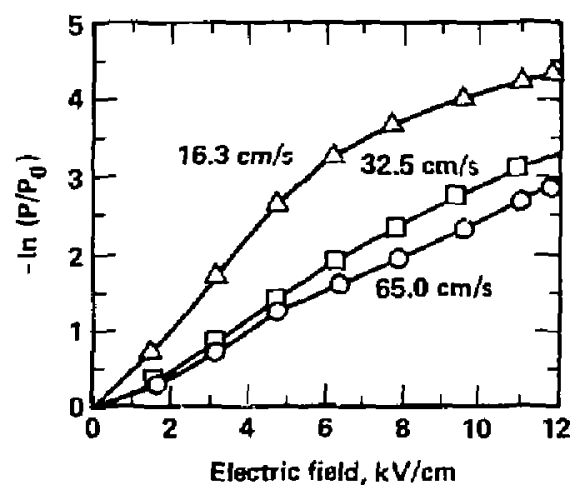

(b)

Fig. 50 (a) filter efficiency as a function of electric field for two layers of AF-18 media tested at different flow conditions. (b) Penetration ratio for
the data in (a). 
An alternative method of plotting the Fig. $50(a)$ data is presented in Fig. $50(b)$. Here, instead of platting filter efficiency, the logarithm of the penetration ratio, $P / P_{0}$, is graphed, where $P_{0}$ is the penetration occuring without an applied electric field. Larger values of $-1 n\left(P / P_{0}\right)$ indicate greater filter efficiency. This normalized plot usually results in more linear electric-field dependencies. As we will discuss in the next section regarding theory, this type of plat, in effect, cancels the mechanical capture mechanisms, leaving only the electrical mechanism.

Similar enhanced relationships are shown in Figs, 51 and 52. Higher packing density fraction means greater efficiency and smaller diameter of the fibers also mean greater efficiency. In Fig. 51, we illustrate the effects of placing one and two layers of AF-18 media between 1.27 cm-spaced screens at a face velocity of $65 \mathrm{~cm} / \mathrm{s}$. Compressing the two layers had the net effect of simply doubling the packing fraction and then doubling the slope penetration ratio vs electric field curve. To separate the effect of fiber size on the filter performance, we tested comparatively using $A F-4$ and $A F-1$ l media having fiber diameters of $0.89 \mu \mathrm{m}$ and $2.6 \mu \mathrm{m}$, respectively. The penetration ratios in Fig. 57 have been normalized to the packing fraction since the AF-4 has a higher volume of fibers than the AF-11 media.

Conductivity. The conductivity of the fiber is an important variable in electric air filtration since it determines the amount of charge that can be accumulated on the fibers and the associated parcicle load on the filter or the acid coats on the fiber surface. Both effects may lower filter efficiency. The effect of fiber conductivity on filter efficiency also depends upon the insulation of the high-voltage electrodes. Figure 53 shows the results from efficiency tests on a conducting filter using both insulated and noninsulated electrodes. Again, the logarithm of the penetration ratio is plotted against the applied electric field to linearize the resulting curve and eliminate the initial filter efficiency without an external field. We see that increasing the electric field improves the fiTter efficiency when noninsulated electrodes are used but has no effect when insulated electrodes are used.

For tests on a less conductive filter, both insulated and noninsulated electrodes showed an increase in filter efficiency when the applied electric field is increased (Fig. 54). However, filter efficiency was lower when an insulated electrode was used instead of a noninsulated electrode. Based on the results shown in Figs. 53 and 54 , we would have expected that, in the 


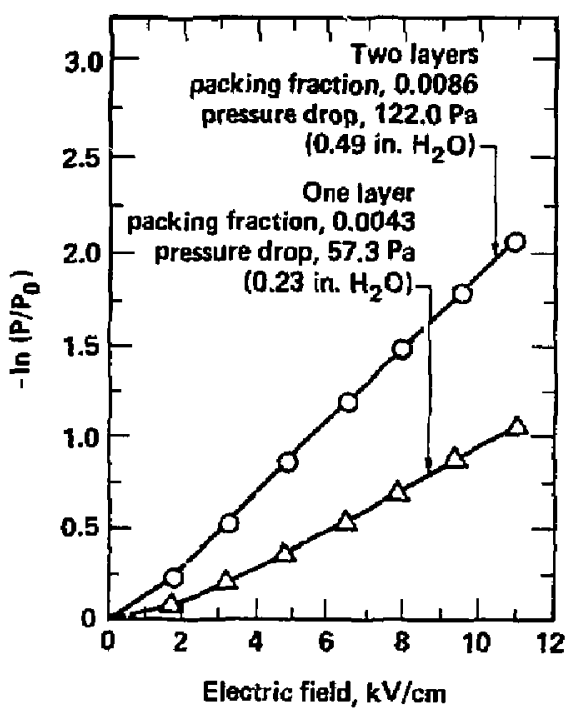

Fig. 51 Filter penetration as a function of the electric field for two filter packing fractions.

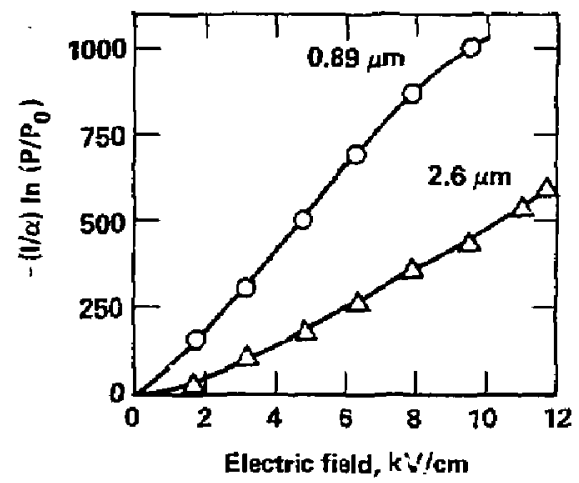

Fig. 52 Normalized filter penetration as a function of electric field for filters having two different fibe; diameters. 


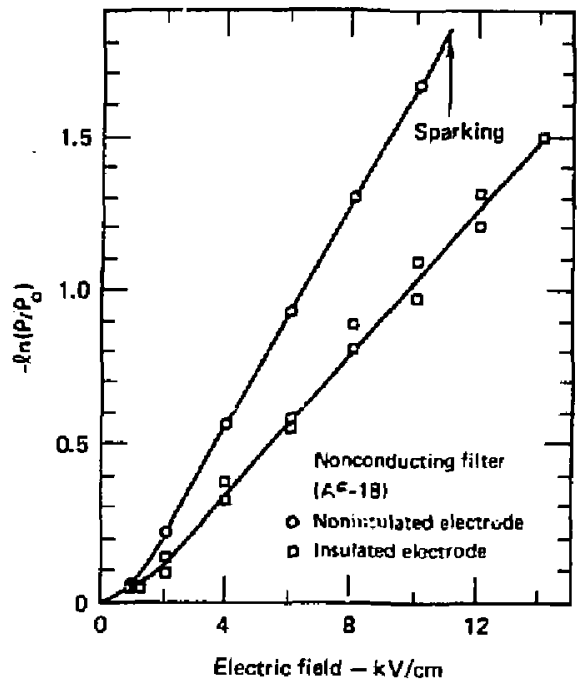

Fig. 53 Effect of Electrode insulation on the enhanced filtration for a conducting filter.

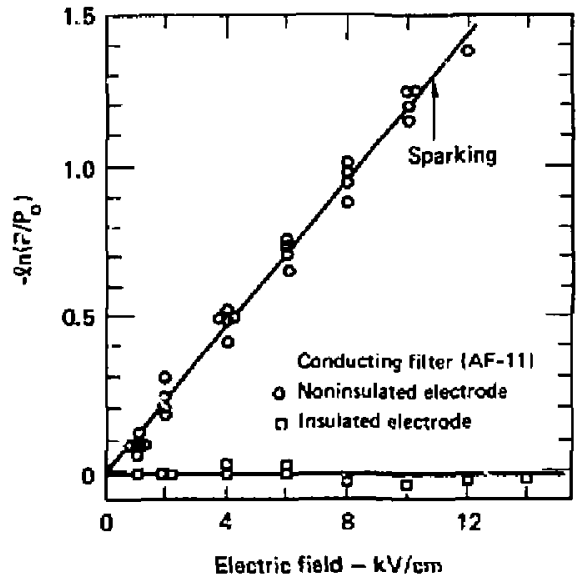

Fig. 54 Effect of electrode insulation on the enhanced filtration for a nonconducting fiTter. 
1 imit of zero conductivity, the filter efficiency would have been the same for either insulated or noninsulated electrodes. The conductivity of the fibers did not have to be very large for the filter to behave as seen in Fig. 53 . The resistivities of the conducting and nonconducting filters were $10^{8}$ and $10^{10} \mathrm{ohm} / \mathrm{cm}$, respectively. Figures 53 and 54 also show that sparking across the electrodes occurs at $11 \mathrm{kV} / \mathrm{cm}$ for the noninsulated electrodes while no sparking occurs for the insulated electrodes up to $14 \mathrm{kV} / \mathrm{cm}$.

The test results above can be explained in terms of a charged-fiber mechanism. Figure 55 shows a filter mat of fibers sandwiched between two electrodes that are connected to a high-voltage source. The high voltage produces an electric field, $E_{0}$, within the filter bed that polarizes individual fibers. Charged aerosol particles are then attracted to the polarized fibers, and, if the fibers have a low conductivity, accumulate a net charge on the fibers. However, since all materials have a finite conductivity, the charge on the fibers will migrate in the direction of the electric field and eventualy dissipate with time. The positive and negative charges migrate along the fibers to the electrode of opposite charge and build up an induced charge.

If the electrodes are not insulated, these induced charges are neutralized on contact with the electrodes. If the electrodes are insulated, the induced charge cannot be neutralized. These induced charges then generate an electric field that is opposed to the external field created by the high-voltage electrodes. The net effect is a reduction of the electric field within the filter medium. The extent of this field reduction depends or the magnitude of the induced charge. For the filter shown in Fig. 54, the low conductivity allows only a small charge to build up next to the insulated electrode. This small charge buildup generates a small opposing electric field that consequently reduces filter efficiency by only a small amount. is contrast, the conducting filter of Fig. 54 allaws a significant charge to build up next to the insulated electrodes. The magnitude of this induced charge is large enough to cause a net cancellation of the external electric field within the filter medium. In this case, an applied electric field does not increase the filter efficiency.

Transient Behavior. The efficiency measurements discussed so far were obtained under steady-state conditions. Experiments conducted under transient conditions (Figs. 56,57,58) demonstrate that the characteristics of the 


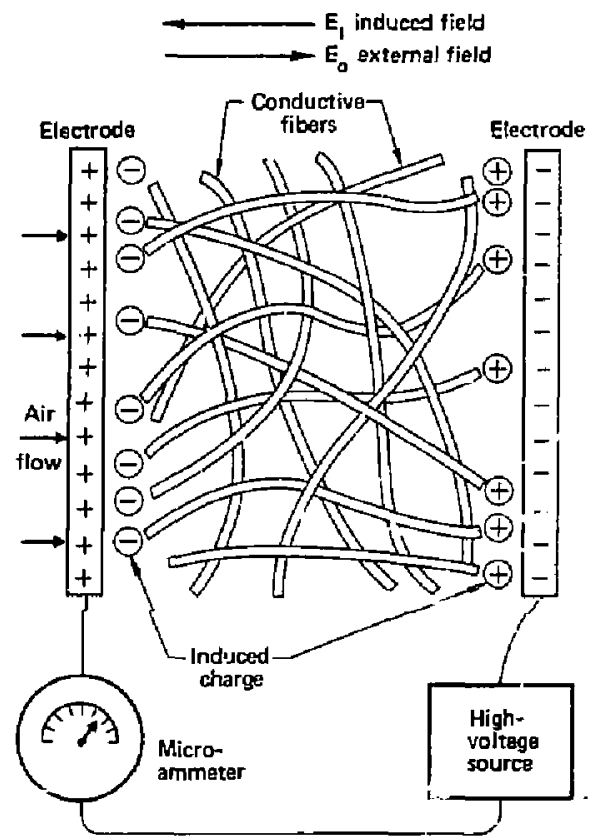

Fig. 55 Schematic illustrating the induced charge formation that caricels the applied electric field when a conducting filter is used wit: insulited

Q lectrodes.

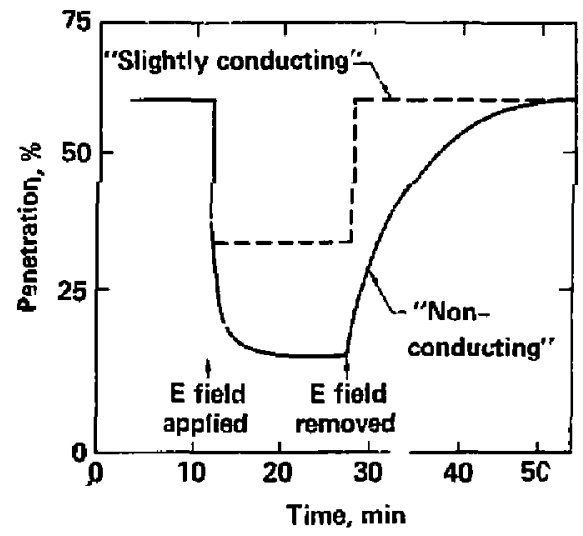

Fig. 56 Transient filter penetration with a "nonconducting" and a "s i ightly conducting" filter. The use of $A C$ voltage produced similar results as the slightly conducting media. 


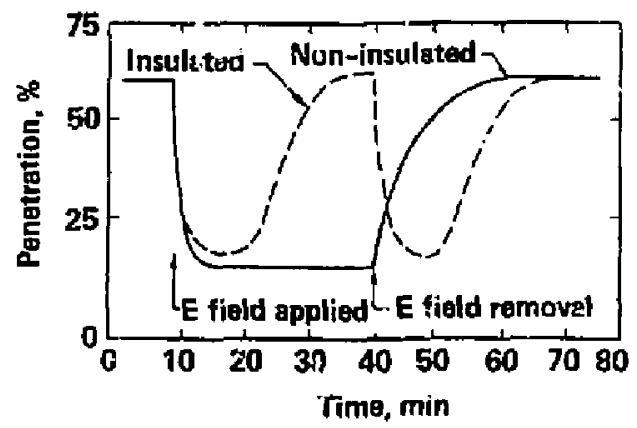

iio. 57 Transient filter penetration for "ncnconducting" filters using insulated and noninsulated electrodes.

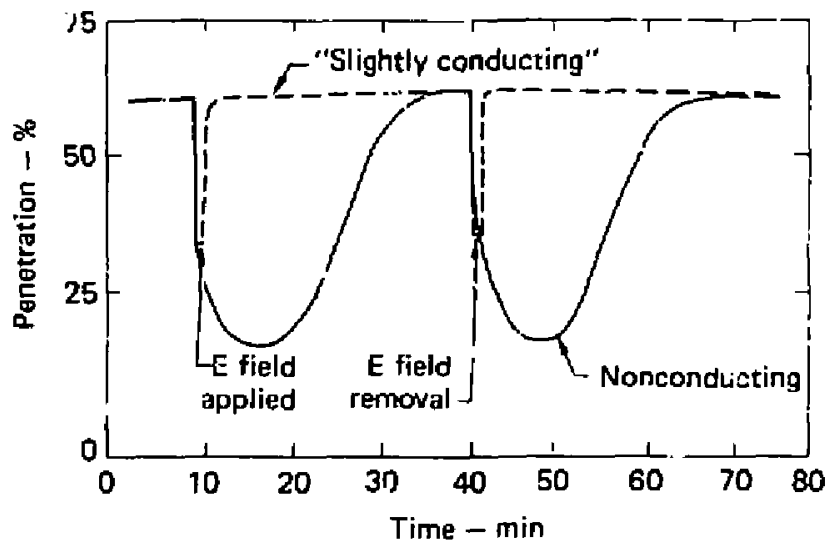

Fig. 58 Transient filter penetration for "nonconducting" and a "slightily condusting" filter using insulated electrodes. 
electrification process are much more complex than previously thought. 23,42 Transient penetration tests can provide additional information on the dynamic nature of the fiber charge. Figure 56 shows the results of two experiments superimposed on the same graph in which the transient aerosal penetration was measured as the electric field was turned on and off. The noise in the original traces was averaged out to yield the smooth lines. The filter designated as "slightly conducting" showed an instantaneous decrease and increase in the penetration when the electric field was turned on and off. In contrast, the "non-conductivity" filter showed a pronounced delay in the penetration when the electric fieid was turned on and off. This delay was much greater when the electric field was removed than when it was applied.

The major difference between the two filters was their conductivity: the "non-conducting" and "lightly conducting" filters had gross resistivities of $10^{10} \mathrm{ohm} / \mathrm{cm}$ and $10^{8} \mathrm{ohm} / \mathrm{cm}$, respectively. Further cases of transient behavior are shown in Figs. 57 and 58 . Figure 57 compares the penetration of a filter having "non-conductivity" fibers placed between either insulated or noninsulated electrodes. As previously des:ribed, the noninsulated-electrode case rapidly reached a steady-state penetration level after application of the electric field. However, for the insulated electrode case, the nonetration decreased to a minimum and then increased to the original penetration even with the field still being applied. In Fig. 58, we examined the effect of filter conductivity when insulated electrodes were used. The primary effect of the increased conductivity was a significant reduction in the transient behavior that occurred for the insulated electrode case. The "sightly conductive" filter has a muci higher minimum penetration than the "non-conducting" filter. The use of $A C$ voltage produced similar results as slightly conducting media.

Resuits of our transient penetration studies, including studies with $A C$ fields, are sumarized in Table 5 . Our transient experiments will be compared to theory in the next section, but, because important experimental data, such as particle size and charge and the fiber charge are not yet available, we have intentionally left che description of these these studies simple and qualitative. In any case, experimental results indicate that gooo electrical contact between the electrodes and the filter media is an important criteria fo:" the design of electric air filters.

Relative Humidity. It is we 11 established that water absorbed on an insulator will dramatically increase its surface conductivity. As might be 
Table 5. Theoretical model of conductivity filter experiments.

\begin{tabular}{|c|c|c|c|c|c|c|}
\hline \multirow{4}{*}{ Electrode } & \multirow{3}{*}{\multicolumn{3}{|c|}{ Experimental }} & \multicolumn{3}{|c|}{ Theoretical } \\
\hline & & & & \multirow{3}{*}{$\begin{array}{l}\text { Fiber } \\
\text { charge }\end{array}$} & \multirow{3}{*}{$\begin{array}{c}\text { Charge } \\
\text { accumulation } \\
\text { at electrode }\end{array}$} & \multirow{3}{*}{$\begin{array}{l}\text { Polarizod } \\
\text { fiber }\end{array}$} \\
\hline & & & & & & \\
\hline & E-field & Fiber & Efficiency & & & \\
\hline \multirow[t]{2}{*}{ Non-insulated } & $D C$ & Non-conduct ing & $\begin{array}{l}\text { Large enhance- } \\
\text { ment, Slow } \\
\text { transient }\end{array}$ & Large & No & Yes \\
\hline & $D C$ & $\begin{array}{l}\text { Slightly } \\
\text { conducting }\end{array}$ & $\begin{array}{l}\text { Moderate enhance- } \\
\text { ment, Fast } \\
\text { transient }\end{array}$ & Sma11 & No & Yes \\
\hline \multirow[t]{2}{*}{ Insulated } & $O C$ & Non-conducting & $\begin{array}{l}\text { No enhance- } \\
\text { ment, slow } \\
\text { transient }\end{array}$ & Mone & Yes & no \\
\hline & OC & $\begin{array}{l}\text { SIightly } \\
\text { conducting }\end{array}$ & $\begin{array}{l}\text { No enhance- } \\
\text { ment, Fast } \\
\text { transient }\end{array}$ & None & Yes & No \\
\hline Both & $A C$ & Both & $\begin{array}{l}\text { Moderate enhance- } \\
\text { ment, Fast } \\
\text { transient }\end{array}$ & None & No & Yes \\
\hline
\end{tabular}


expected, high humidities can lead to increased current flow through the filter and eventually short-circuit the electrodes. The impact of relative humidity on the filter efficiency and electrode is illustrated in Fig. 59. Since $\mathrm{NaCl}$ aerosols are hydroscopic, DOF nerosols were used for these tests with a light-scattering photometer on the detector. The filter efficiency with and without high voltage remains constant over all relative humidities tested; below $70 \%$ relative numidity, the electrode current remains less than $0.5 \mu \mathrm{A}$. Beyond $80 \%$ relative humidity, the current rapidly increases until there is a short-circuiting across the electrodes at $90 \%$ for $g$ lass (e.g., polypropylene absorbs about $0.02 \%$ water as compared to $0.3 \%$ for glass). We would expect that electric aiw filters made from plastic fibers can operate at a much higher relative humidity.

\section{SIMPLIFIED FILTER EFFICIENCY THEORIES}

The electric air filter is a complex, dynamic system involving both mechanical and electrical forces. For example, increasing the face velocity increases the filter efficiency for mechanical capture mechan sms, but decreases the filter efficiency for electrical mechanisms. Tr a air flow resistance or pressure drop across a filter is unaffected by the aditition of an electric field. This follows since air-flow resistance is dependent on physical obstruction, not electrical ones.

Since the efficiency of an electric air filter depends upon both mechanical and electrical mechanisms, both must be considered in a theory. We $\therefore$ veloped theoretical models for filter efficiency that are consistent with the experimental results presented in the previous section. The simplified theories discussed here explain both the enhanced efficiency and transient behavior of electric air filters. They are applicable to clean filters only; theoretical models applicable to mass-loading will be discussed later in the section Effects of Particle Loading on Filter Eff iciency.

\section{Steady-State Penetration Theory}

The most frequently used approach for investigating microscopic filtration mechanisms in fibrous filters is the concept of singie-fiber efficiency. This concept assumes that a single fiber is representative of all fibers in the 


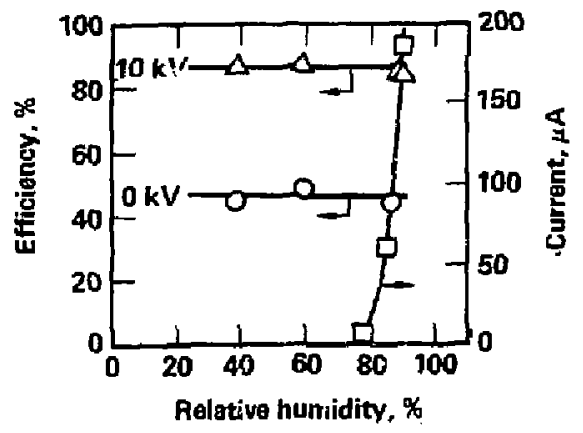

rig. 59 Filter efficiency and electrode current as a function of relative humidity for an electrofibrous filter. 
fijr $r$. Singie-fiber efficiency takes into account ali interactions between neighboring fibers and should be not confused with the efficiency of an isolated single fiber. The differential form for this filtration model is

$-\frac{\partial N}{\partial X}=\frac{2 \alpha N_{\eta_{T}}}{\pi R(1-\alpha)}$

where $N$ is concentration $\left(\mathrm{cm}^{-3}\right)$ of particles, a is the volume fraction of fibers, $X$ is the filter thickness $(\mathrm{cm}), \pi_{T}$ is the total single-fiber efficiency, and $R=$ fiber radius $(\mathrm{cm})$. Using this formula, we can derive the integrated expression that relates the observed filter penetration, $P$, to the single-fiber efficiency, $n$,

$P=e^{-\frac{2 a X}{(1-a) \cdot R^{m}}}$

Tile filter efficiency, $E$, is related to the penetration by

$E=1-\square$

The single-fiber efficiency, $\pi$, in Eq. 31 represents all of the mechanical and electrical mechanisms for particle capture superimposed on the air flow through the fiber matrix. The most rigorous derivation of $n_{T}$ combines the three mechanical capture mechanisms of diffusion, interception, and inertial impaction with the Kuwabara flow-cell model. ${ }^{43}$ Although the resulting equation has predicted many experimental trends, inuch additional work is still required before quantitative predictions cal, be made for real filters.

An important finding of these theoreticai investigations is that the efficiency of individual capture mechanisms cannot be added, d priori. together to yield the combined efficiency for all mechanisms. Theoretical studies show that the total mechanical efficiency equals the sum of the individual efficiencies plus an additional term involving both diffusion anc interception. It seems reasonable to expect additional mixing of terms when electrical mechanisms are added to the filtration model. Since the derivation of ${ }^{n} T$ for the combined electrical and mechanical mechanisms superimposed 
on the air-flow pattern is not yet available, we shall approximate it with the sum of the efficiencies for the mechanical, $n_{M}$, and individual electrical mechanisms, $n_{j}$ :

$$
\eta_{T}=\eta_{M}=\sum_{i} \eta_{i}
$$

Previous theoretical and experimental studies have identified two mechanisms responsible for the increased efficiency resulting from an applied electric field: the attraction between polarized particles and polarized fibers and the attraction between charged particles and polarized fibers. The external electric field polarizes both fibers and particles. We have ignored the additional capture mechanism due to attractiveforces between charged particles and neutral fibers because it is a minor contribution to the filter efficiency for naturally charged aerosols.

Zebe $1^{23}$ derived the foilowing two equations for single-fiber efficiency that represent the force between polarized particles and polarized fibers, ${ }_{n R P}$, and the force between charged particles and polarized fibers, ${ }_{\eta} R, \mathrm{P}_{\mathrm{q}}$. Equations 35 and 36 represent the electrical capture mechanisms for particles in the interception region:

$$
\eta_{R, p_{p}}=\left(\frac{\epsilon_{\mathrm{p}}-1}{\epsilon_{\mathrm{p}}+2}\right)\left(\frac{\epsilon_{\mathrm{F}}-1}{\epsilon_{\mathrm{F}}+1}\right) \frac{\tau^{2} \mathrm{E}^{2} \mathrm{C}}{\epsilon-\mu R v} \text { and }
$$

$\eta_{R . P_{q}}=\frac{\operatorname{neEC}}{6 \pi \mu v r}\left[\frac{1+\frac{\epsilon_{F}-1}{\epsilon_{F}+1}}{1+\frac{n e E C}{6 \pi \mu r v}}\right]$,

where $\varepsilon_{p}$ is the dielectric constant of the particle, $\varepsilon_{F}$ is the dielectric constant of the fiber, $r$ is the particle radius, $E$ is the effective field strength, $C$ is the Cunningham slip-ccrrection factor, $v$ is the air velocity inside the filter, $r i$ is the number of elementary charge units, $e$ is the elementary uırit of charge, and $\mu$ is the gas viscosity. 
Zebe $1^{44}$ also derived an equation for the single-fiber efficiency resulting from the diffusion of charged particles to a fiber polarized by an electric field:

$\pi_{D, P_{\mathrm{q}}}=\frac{\mathrm{gneEC}}{9 \mu \mathrm{rV}}$

The value of $g$ in Eq. 37 depends upon regions of attraction and repulsion on the fiber. Note the single.fiber efficincy resulting from the electrical force between charged particles and polarized fibers is very similar for both interception and diffusion mechanisms. Since particles in the diffusion region are very difficult to polarize because of their small size, an efficiency term analogous to Eq. 35 that involves forces between polarized particles and polarized fibers would not be important. One additional efficiency term, ${ }_{I}, P_{p}$, has been identified for the theoretical electrostatic filter.

Al though no one has yet determined this terin, it is applicable in the region of inertial impaction and will most likely be dominated by the attraction between polarized particles ano polarized fibers.

Adding together all of the single-fiber efficiencies due to mechanical and electrical mechanisns, as shown in Eq. 34, the total single-fiber efficiency is

$\eta_{T}=\eta_{M}=\eta_{R, P_{P}}+\eta_{R, P_{q}}+\eta_{D, P_{q}}+\eta_{1, P_{q}}$

For comparison to experimental measurements, we have dropped the inertial term and substituted Eq. 32 into the expression for penetration. Oividing the resulting penetration equation by the initial penetration without an electric field, $P_{0}$, eliminates the mechanical efficiency terms and yields a penetration ratio that is dependent on the applied electric field. The logarithm of this penetration ratio is

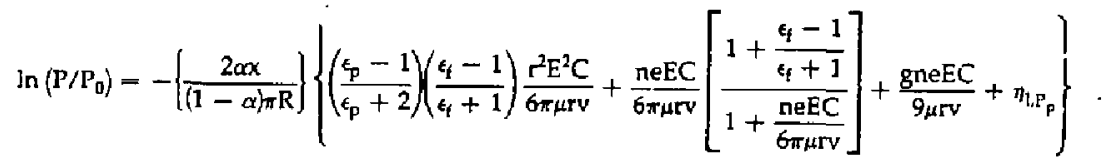


We calculated theoretical curves using Eq. 39 without the last two singlefiber efficiency terms. The definition of terms and vaiues used in these calculations are listed in Table 6 . The results in Figs. 60-64 show the dependence of the penetration $r a t i o,-\ln \left(P / P_{0}\right)$, or the applied electric strength for a variety of parameters affecting filter perfomance. These dependencies include face velocity, packing dersity, fibar size, particle size, and particle charge, which we have already discussed. The greater the slope of any curve, the more dependent fitter performance is on the variable being compared. We wlll next compare these dependencles with the experimental results described previuusly.

Figure 60 illustrates the penetration ratio for three common face velocities. Note that lawer face velocities yield higher filter efficiencies. These calculations can be compared to our experimental findings shown in Fig. 65. Here, the lines are nearly linear except for the curve at $153 \mathrm{~cm} / \mathrm{s}$ at higher $\mathrm{E}$ fields, indicating that for the $\mathrm{NaCl}$ aerosol, the primary electrical capture mechanism is Coulombic. [Eq. 36], rather than the polarization term in Eq. 35. According tc Eq. 36, the polarization term would make the data increase as $E^{2}$, which clearly does not occur.

The fitter performance calculated for four different packing fractions is shown in Fig. 61. Not surprisingly, the behavior of this parameter is entirely consistent with experiments shown in Fig. 51, that is, doubling the packing fraction doubles the log ratio of filter penetration. Of course, for a given flow, the increased packing fraction will also increase the pressure loss across the filter.

Filter performance at various fiher diameters is illustrated in Fig. 62 for a constant packing fraction. Performance greatiy increases as fiber size is reduced. This can be compared to the experimental results shown in Fig. 52, where the penetration ratio has been normalized to remove the effects of different packing densities.

The dependence of filter penetration upon particle diameter is more complex. The family of theoretical zurves drawn in Fig. 63 depicts the ratios : or different particle sizes having the same charge, $5 \mathrm{e}^{-}$. At any fixed electric field, the filter efficiency first decreases to a minimul, and then increases as the particle size increases. The trends are best understood by referring to Eq. 39 in which the polarization term varies as the square of 
Tabie 6. Parameters used for the theoretical calculations.

\begin{tabular}{ll} 
Media & Johns-Manvilie 1.27-cm Microlite (AF-18) \\
Layers & 2 \\
Packing fraction, $\alpha$ & 0.086 (fi)ter weight $10.8 \mathrm{~g}$ ) \\
Thickness, $\mathrm{x}$ & $1.27 \mathrm{~cm}$ \\
Fiber radius, $\mathrm{R}$ & $4.5 \times 10^{-4} \mathrm{~cm}$ \\
Face velocity, $v$ & $65 \mathrm{~cm} / \mathrm{s}$ \\
Particle dielectric constant, $\varepsilon_{\mathrm{p}}$ & 6.12 \\
Fiber dielectric constant, $\varepsilon_{\mathrm{f}}$ & 3.87 \\
Particle charge, $\mathrm{n}$ & $5 \mathrm{units}$ \\
Particie diameter, courit median, $2 \mathrm{r}$ & $0.29 \times 10^{-4} \mathrm{~cm}$ \\
Electric field, E & $0 \mathrm{to} 12 \mathrm{kV} \mathrm{cm}$ \\
Cunningham slip correction, $\mathrm{C}$ & 1.66 \\
Air viscosity, $\mu$ & $1.8 \times 10^{-10} \mathrm{Pas}$ \\
Electronic charge, e & $4.8 \times 10^{-10} \mathrm{esu}$ \\
\hline
\end{tabular}




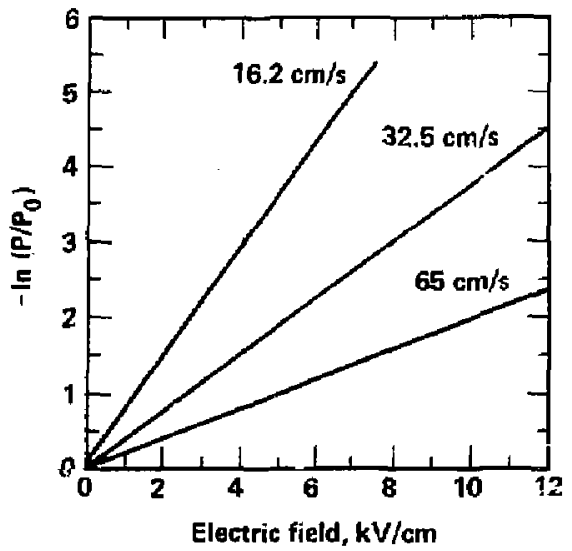

Fig. 60 Theoretical filter penetration as a function of electric field for three face velocities.

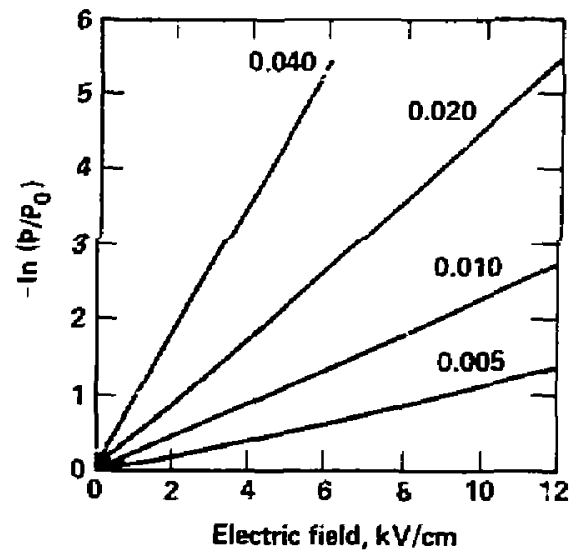

Fig. 61 Theoretical filter penetration as a function of electric field at various filter packing fractions. 


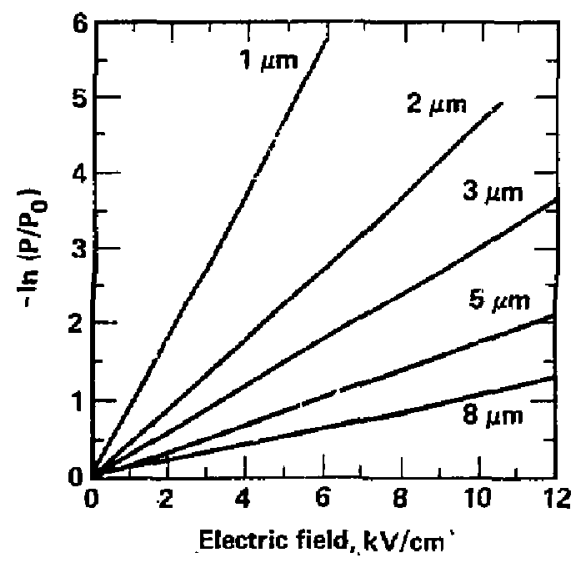

Fig. 62. Theoretical filter penetration as a function of electric field for fibers of varying diameters.

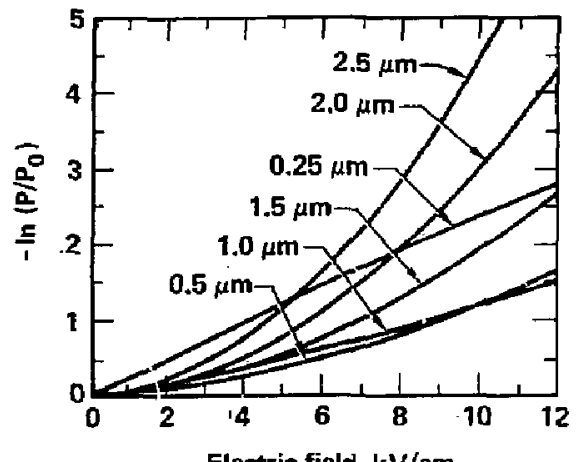

Electric field, $\mathrm{kV} / \mathrm{cm}$

Fig. 63 Theoretical filter penetration as a function of electric fiiter field for various size particles. 


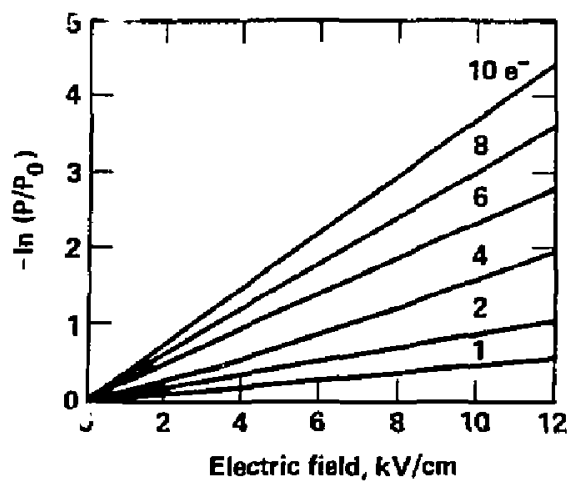

Fig. 64 Theoretical filter penetration as a function of electrical field for particles of varying charge.

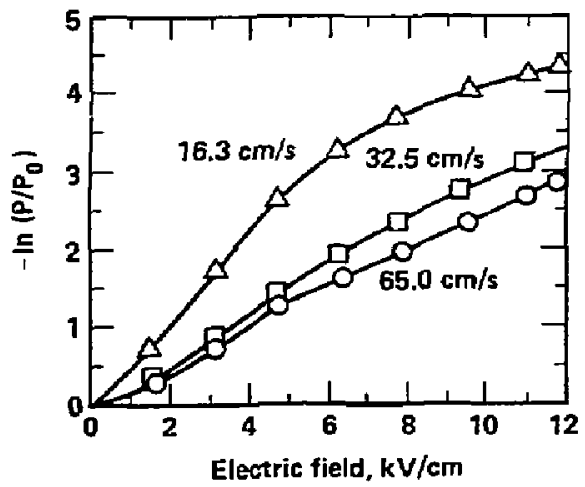

Fig. 65 Penetration ratio for various electric fieids at three face velocities. 
ciameter, whereas the Coulombic term varies in the first approximation as the inverse diameter. Thus, smalier particles interact. pirmarily in a Coulambic manner, while larger ones show polarization behavior.

The effect of particle charge on the penetration ratio as predicted by this made; is illustrated in Fig. 64. As the particle charge increases, the filter-collection efficiency also increases. Calculations were made for 0.29-um diameter jarticles. The dependence of the pcretration on size and charge has not yet been compared to experfmental resuics because the size-charge instrumentation is still being developed.

In general, our comparisons between Zebel's theory and our experimental observations indicate qualitative agreenent:. The parameter packing density, fiber size, face velocity, and particle size foilow the trends predicted from the theoretical relationship. This implied model assumes steady-state conditions and the aspects of mechanical capture mechanisins having been avoided by using penetration ratios. The dependence on particle charge and electric field need further investigation on particle charge measurements are routinely operable. Furthermore, for absolute comparisons a more rigorou theoreticai treatment is required.

\section{Transient Penetration}

All of the previous experimental and therretical investigations have assumed that an applied electric field would quickly ircrease the filter efficiency with little or no transient effects. This assumption was supported by a general concensus among investigators that the fundamental mechanisms embodied in Eq. 39 sufficiently described the increased filter efficiency of the electrostatic filter. According to Eq. 39, changes in the applied electric field would result in instantaneous cilanges in the filter efficiency. However, measurements in Figs. 64-66 indic :e the presence of transients that are not predicted by the steady-state theory. Based on these measurements, we concluded that an additional capture mechanism must be added to the theory. The basis for this additional mechanism is 11 lustrated in Fig. 66 .

The larger and smaller circles in Fig. 66 represent the cross sections of the fibers and the charged particles, respectively. The salid lines are the electrical lines of force, whtle the dashed iines are the particle trajectories. Although only the attraction for charged particles is included, 


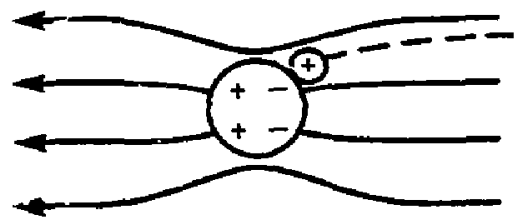

Polarized fiber - cherged particle

(fiber polarized by electric field)

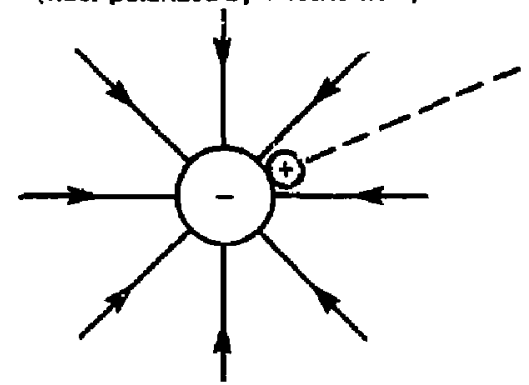

Charged fiber - charged particle

(fiber charged from the deposition

of charged particles)

Fig. 66 E ctrical capture mechanisms responsible for the increased filter efficiency. 
the electrical capture mechanisms are equally val id for polarized particles. The particles can be polarized by either the fiber charge or by the external electric field.

When an external electric field is first applied to the filter, the only capture mechanism is due to the forces between a polarized fiber and a polarized or charged particle. The electric field instantly polarizes the fiber, which then attracts both charged and polarized particles. Zebel had derived Eqs. 35 and 36 based on this capture mechanism. However, the charged particles that deposit on the fiber gradually build up a fiber charge, and, therefore, introduce the second mechanism. This second mechanism is based on the force between charged fibers and charged or polarized particles.

The fiber with $q$ charge per unit length can subsequently capture charged or neutrat particles. The charged particles are attracted by Coulombic forces, while the neutral particles are attracted by induction forces. For charged particles having a charge, ne, the single-fiber efficiency is 45

$$
\eta_{\mathrm{R}, Q_{\mathbf{p}}}+\frac{\mathrm{neQC}}{3 \mu \mathrm{r} \mathrm{R}_{\mathrm{V}}}
$$

The corresponding single-fiber efficiency for neutral particles is

$$
\eta_{R Q_{p}}=\frac{2}{3}\left(\frac{\epsilon_{\mathrm{p}}-1}{\epsilon_{\mathrm{p}}+2}\right)\left(\frac{\mathrm{Q}^{2} \mathrm{r}^{2} \mathrm{C}}{\mathrm{R}^{3} \mu \mathrm{v}}\right)
$$

We should note that the induction force on the neutral particles in Eq. 41 is produced by the fiber charge, $Q$. As in the case of the steady-state theory, additional mechanisms are also required ior the electrical capture mechanisms in the diffusion and inertial impaction regions of particle size. Zebel has derived the following expression for the combined mechanisms of diffusion and Coulombic attraction between charged particles and charged fibers ${ }^{44}$ :

$$
\eta_{D, Q_{q}}=\frac{g m e Q C}{g \mu r R V}
$$


Although the single-fiber efficiency for charged fibers and polarized particles in the inertial region, ${ }^{I}, Q^{p}$, has not yet been derived, it is expected to resemble Eq. 31 , for the interception region.

The neutral particle can also be polarized by the external electric field; in which case, the single-fiber efficiency is proportional to $Q E$ instead of $Q^{2}$. A similar cross term occurs when the fiber has a charge and is also polarized by the external electric field. In this case, a neutral particle can be polarized either by the fiber charge or by the electric field. Equation 25 represents the case in which the particle is polarized by the external field. If the particle is polarized by the fiber charge, then tire single-fiber efficiency term describing the attraction between polarized particles and polarized fibers is proportional to $Q E$. Therefore, once the fiber becomes charged in the presence of an external electric field, two additional single-fiber efficiency terms involving $Q E$ are added to the combined fiber-collection efficiency. Including these terms and replacing the fixed variables in all of the single-fiber efficiencies with constants, the resulting equation describes the differential decrease in the number of particles, dN, across a filter element of differential thickness, $d x$, which is given by

$-\frac{d N}{d X}=\frac{2 \alpha N}{\pi R(1-\alpha)}\left[ग_{M}+C_{1} n e E+C_{2} E^{2}+C_{3} n e Q+C_{4} Q^{2}+C_{5} Q E\right]$

We have assumed that inertial-impaction terms have dependencies similar to those of the interception region. The fiber charge $Q$ is given by

$\frac{d Q}{d t}=-\frac{\gamma Q}{t}-n e v \frac{d N}{d x}$

Ea. 34 is the sum of a dissipation term, $-r Q / E$, and an accumulation term, $-n e V d N / d x$. Equations 33 and 34 represent a pair of coupled differential equations.

Although extensive numerical calculations are required to compute trarisient and steady-state penetrations, we can qualitatively explain experimental trends using lq. 34. The transient test results shown in 
Fig. 56 indicate that increased conductivity removes large transients in filter penetration that occur for nonconducting filters when the electric field is applied and removed. The explanation for the conductivity effect is based on Eq. 34. For a nonconducting filter, small $r$, a significant charge can build up on the fiber before the charge-dissipation term, $-Y Q / E$, reaches equilibrium with the charge-accumulation term, -neVdN/dx. However, the charge-dissipation term for the conducting fiber, large $\gamma$, is so large that equilibrium between charge dissipation and accumulation is established with a negligible charge on the fibers. Since tine conducting fiber cannot develop a charge, the penetration will instantiy increase to the initial penetration when the electric field is turned off.

In contrast, the nonconducting filter has a significant fiber charge which bleeds off very slowly when the electric field is turned off. As a rough appreximation, the accumulation term in Eq. 34 can be ignored and the resuiting equation integrated to yield

$\mathrm{Q}=\mathrm{Q}_{\mathrm{ss}} \mathrm{e}^{\frac{-\gamma_{\mathrm{t}}}{\mathrm{t}}}$

where $Q_{s s}$ is the steady-state charge before the electric field is removed.

In a more rigorous analysis, the accumulation term in Eq. 34 does not reduce to zero because it is a function of the fiber charge. This resuits in a longer time for the charge to bleed off. Although the polarized fiber mechanism disappears imediately when the electric fieid is removed, the charged-fiber mechanism persists until all of the charge bleeds off.

Figure 67 sumrarizes the contribution of the two theoretical mechanisms to the transient filter penetrations seen in our experiments. Figure 67 clearly shows that the experimental transients are due to the charged-fiber mechanism. We grouped all of the differert mechanisms previous ly discussed that invoive charged fibers and charged or polarized particles as charged-iber mechanisms. Similarly, all of the previously discussed mechanisms invalving polarized fibers have also been grouped together. Although a symmetrical transient is indicated for both fiber-charging and discharging, asymetrical transients are predicted when the charged-fibe- and polarized-fiber mechanisms are considered together. Figure 67 also illustrates the large contribution of 


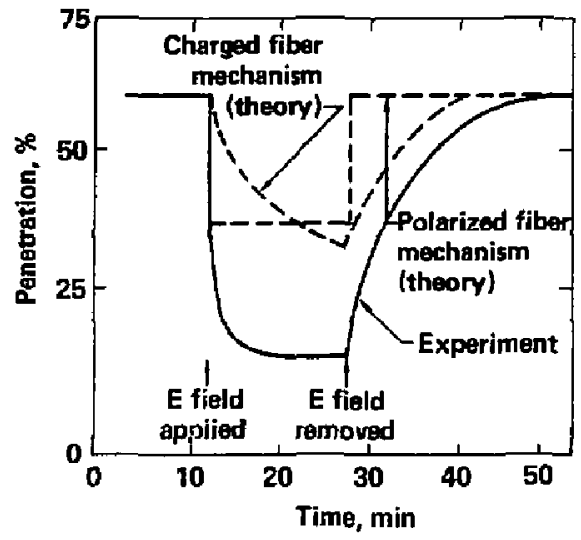

Fig. 67 Contributions of polarized fiber and charge fiber mechanisms to the transient filter penetration. 
both mechanisms to the tota? decrease in filter penetration. Understanding the nature of the charged fiber mechanism is very important in practica] applications because of its large contribution to the filter efficiency.

Dur transient penetration studies have shown that mechanisms responsib]e for the increased efficiency when an electric field is applied across a fibrous filter are more complicated than previously believed. These studies have been qualitative because of insufficient data to establish a quantitative relationship between theory and experiment. Because important experimental data, such as particle size and charge and fiber charge, are nat yet available, the theoretical treatment of the electrical capture mechanisms has been intentionally kept very simple. Nevertheless, our transient penetration can predict the dynamic nature of the accumulation and dissipation of charge on fibers.

\section{RIGOROUS THEORY}

In conjunction with the Mathematics Department of the University of California at Davis, we developed a rigorous filtration theory to predict the enhanced efficiency of electric air filters. In this theoretica? effort, we developed a computer code to determine the filter efficiency, accounting for particle-capture mechanisms of diffusion, interception, and inertial impaction. The forces acting on particles include those resulting from the air-flow field, as modeled by Kuwabara, ${ }^{43}$ and those resulting from all combinations of charged and polarized particles and fibers.

To determine filter efficiencies, the single-fiber efficiency is determined for the general case encompassing all external forces acting on the particle. The single-fiber efficiency is defined as

$\eta=\phi / 2 \mathrm{Rn}_{0} \mathrm{~V}_{0}$

where $R$ is the fiber radius, $n_{0}$ is the incoming particle concentration. $V_{0}$ is the the mean particle velocity at the flow cell boundary, and is the particle deposition rate on the fiber. 
The particle deposition rate is obtained by solving the particle trajectory equation for external forces given by fuchs ${ }^{46}$ :

$m \frac{d \bar{w}}{d t}=-6 \pi \mu r(\bar{w}-\bar{u})+\bar{F}$

where $m$ is the particle mass, $r$ is the particle radius, $w$ is the particle velocity, $u$ is the airstream velocity, and $F$ represents the external forces. The term, $-6 \pi \mu r(w-u)$, is the drag acting on the particle and $F$ are the farces of particle charge, fiber charge, and particle and fiber polarizations due 0 the electric field. No analytical solution for this equation is known in the general case. Therefore, most investigators find it necessary to simulate many numerical particle trajectories via integration of Eq. 37 and accumulate the ones which intercept the fiber. 30,46 particle-deposition rate and single-fiber efficiency are then computed. Methods have been developed in our theory to overcome much of the computational inefficiency in the above "shuoting method."

In the case of the interception/diffusion region for particle collection, we developed a numerical method that requires the solution of only a single, initial-value problem as opposed to the shooting method ${ }^{47}$. Computed singlei iber efficiencies are in excelient agreement with the shooting trajectory method and Zebel's theory ${ }^{23}$ in the diffusion-capture regime for strong electric fields. Furthermore, the new numerical method is $20-50$ times faster than shooting methods, depending upon the method used for trajectory integrations.

In the case of the inertial-efficiency region, we used a perturbation approach 43 to develop a numerical procedure for finding an acceptable approximation to the inertial efficiency that does not use a shooting methodalogy. The perturbation approach is based on a method developed by Stechkina et al. and used in the particle range of maximum penetration. 49 For particles of radius $1 \mathrm{um}$ or less, the observed error using the perturbation technique is approximtely $7 \%$ or less compared to the shooting technique. The average computational time is only $1 / 20$ of that required for the shooting method. 
In this section, we direct most of our attention to air fundamental air studies on filters having permanently charged fibers. Permanently charged electric air filters are typically made from high-resistance polymers with permanent electrostatic charges placed on or near the polymer surface. As in the case of the externally charged electric air fiiters, permenently charged surfaces increase the efficiency of the media by providing electrica? capture mechanisms in addition to the mechanical capture mechanisms of conventional filters.

\section{Background}

Air filters having pemanently charged fibers are an effective means for removing submicron aerosols. The permanently charged surfaces on the fibers add electrostatic collection mechanisms to the mechanical capture mechanisms and increase the performance of the filter without a corresponding increase in pressure orop. Prior to this investigation of permanently charged filters, our study of electric air fiters dealt exclusviely with electrifying conventional glass-fiber filters in an electric field. Previous investigators had shown that permanently charged filters have exceptionaliy high efficiencies, while maintaining a low pressure drop. $17,40,41$ There is also experimental evidence to suggest that filtration of certain aerosols, or exposure of the filters to certain chemicals and environmental conditions, can partially neutralize the fiber charge and cause a significant reduction in filter performance. $17, i, 50$

Our primary incentive for investigating permanently charged filters is iliustrated in Fig. 68. Here we compare efficiency as a function of particle diameter for a standard fibrous filter and for the same filter externally electrified. Figure 68 presents the efficiencies of two permanently charged filters ( $N$. V. Verto Co., Rotterdam, Netherlands), one having a lower pressure drop and one having a higher pressure drop than the standard glass-fibrous filter. The permanently charged filters have comparable efficiencies in the submicron size range as that of the electrified filter generated by high-voltage electrodes. The permanently charged filters achieve the high filter efficiency without the equipment required to electrify a standard 


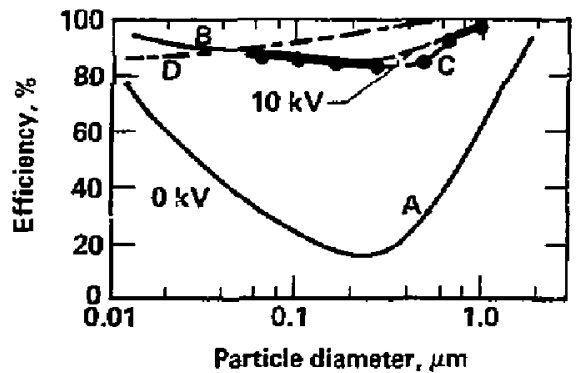

Fig. 69 Efficiency of uncharged, externally charged and permanen'ily charged filters using Nacl aerosols at a face veiocity of $66 \mathrm{~cm} / \mathrm{s}$.
(A) Manville AF-18, $\Delta \mathrm{P}-0.6$ in. $\mathrm{H}_{2} \mathrm{C}$
(B) Manvi?le $A_{i}=18, \Delta P-0.6$ in. $\mathrm{H}_{2} \mathrm{O}$
(C) Filtrete, $200 \mathrm{~g} / \mathrm{m}^{2}, \Delta P-0.6$ in. $\mathrm{H}_{2} \mathrm{O}$
(D) Filtrete, $300 \mathrm{~g} / \mathrm{m}^{2}, \Delta P-0.6 \mathrm{in}$. $\mathrm{H}_{20}$ 
fi]ter namely, a high-voltage power supply, electrodes, and cables.

We evaluated three prominent types of comercially available, pemanently charged filters. Each of the filters is manufactured using a different method for imposing a permanent charge on the fibers. In one method, permanentiy charged filters are generated from electret sheets. ${ }^{40,41}$ A polymer sheet is initially passed across a corona discharge which imparts positive and negative charges on opposite sides of the polymer sheet. In a subsequent step, the sheet is split into small filaments which are formed into a filter mat using conventional technology for non-woven media. Figure 69 shows a series of scanning electron micrographs (increasing in magnification) typical of the permanently charged filter formed from electret fibers (Filtrete).

a second class of permanently charged filters is generated by a technique in which high voltage is applied to a polymer melt during a spinning process

of fiber formation. ${ }^{50}$ This filter is commercially available from the Carl Freudenberg Company, Germany, Micrographs of the Freudenberg filter are shown in Fig. 70. This filter consists primarily of relatively smocth fibers arranged in a random fashion to form a filter mat. Their surface is not as rough as seen in the filtrete media.

The third class of permanently charged filters is manufactured by coating the fiber surface with charged resin particles. This media, commonly called a Hanson filter, is comitercially available from BIS Minerals Limited, London, England as Lapofilters. Electron micrographs of this class of permanently charged filters are shown in Fig. 71. The fibers are heavily coated with resin particles which support all of the charge on the filter media.

\section{Experimental Results}

Figure 72 shows a typical result of the efficiency and pressure drop obtained with the $200 \mathrm{~g} / \mathrm{m}^{2}$ Filtrete media in which two classes of aerosols were loaded onto the filter. We used $\mathrm{NaCl}$ aerosols to simulate a solid-particle loading, and dioctyi sebacate (DOS) aerosols to simulate liquid particie loading. The filter loading with $\mathrm{NaCl}$ aerosol showed an initial decrease in efficiency followed by a rapid increase. We have attributed the initial decrease to the neutralization of the fiber charge by the charged aerosol particles depositing on the filter. The subsequent increase in efficiency is due to the mechanicai capture of new particles by the previousiy 


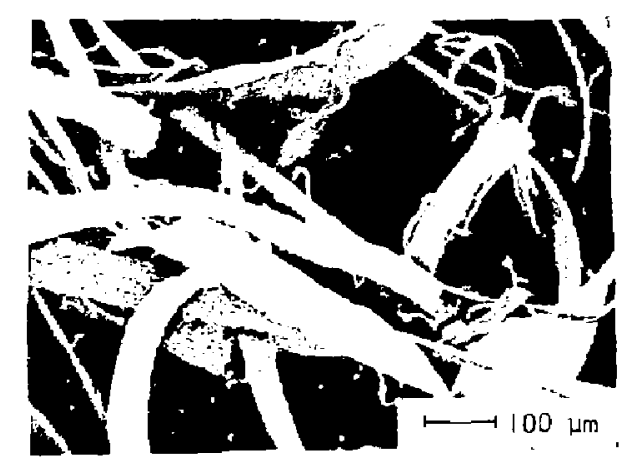

A

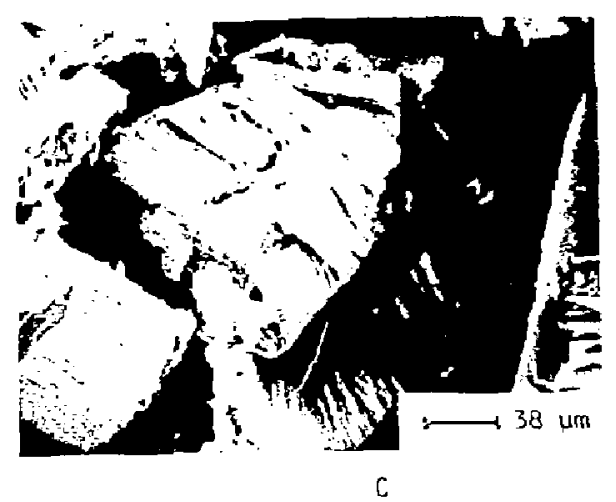

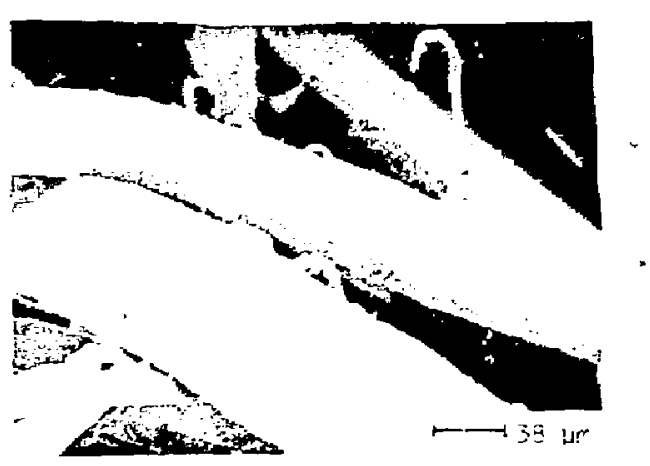

B

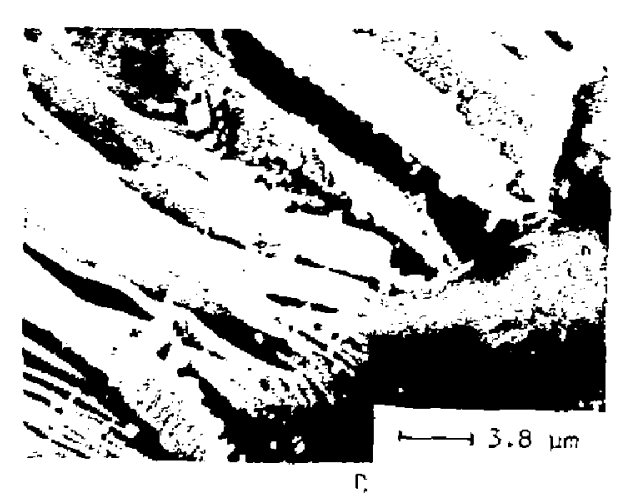

Fig. 69 Electron micrographs of fiitrete filter media. (c) and (d) have collected salt particles on the surface. 

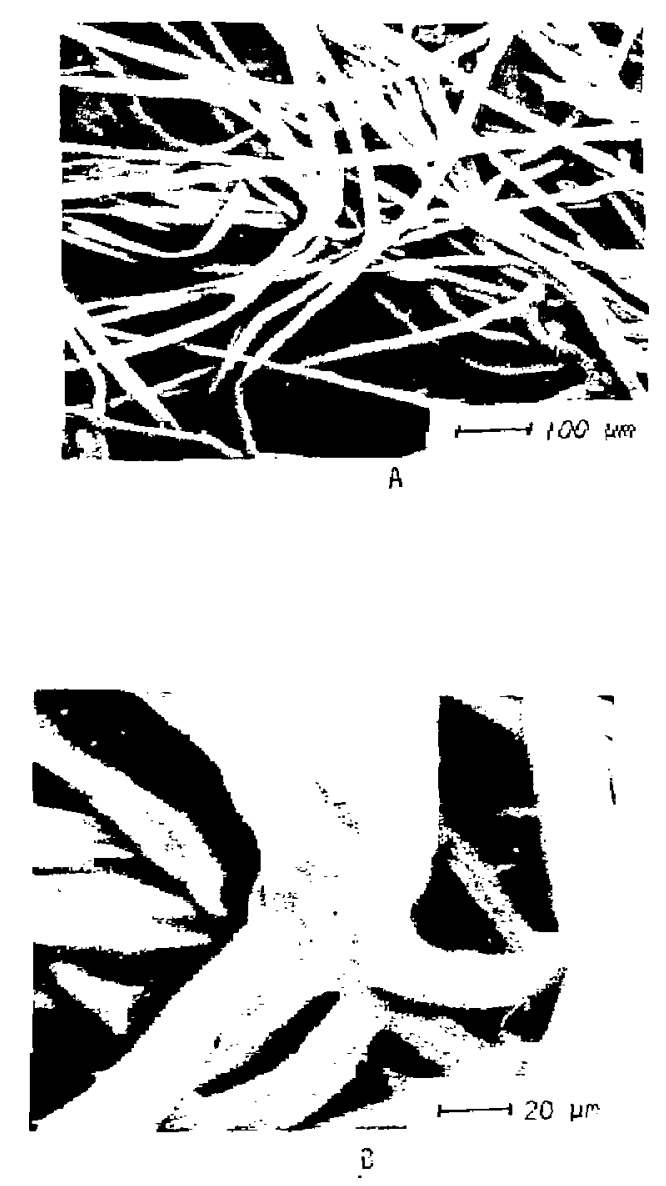

Fig. 70 Electron micrographs of Freudenberg media. 

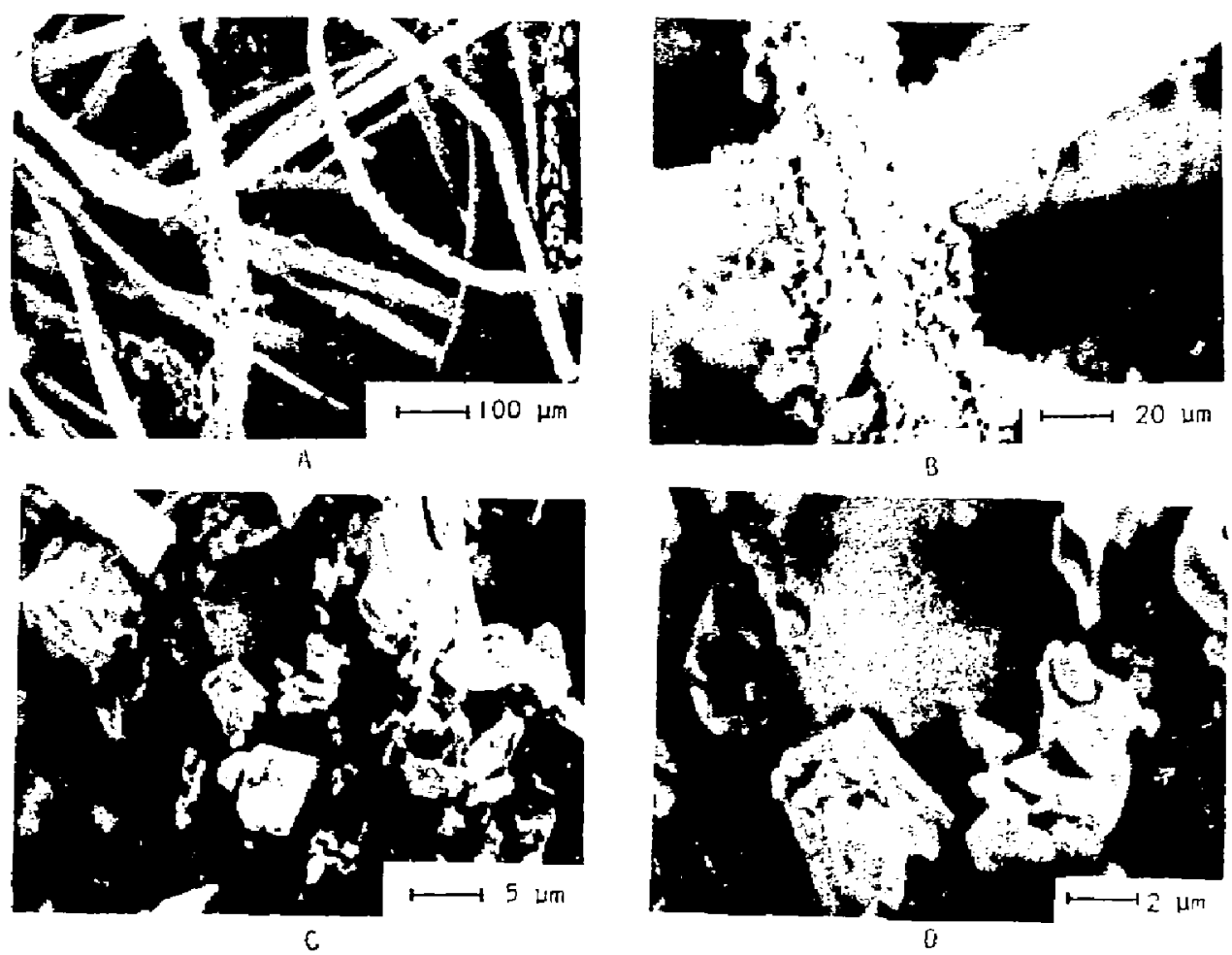

Fig. 71 Electron micrographs of Capofilter media. 


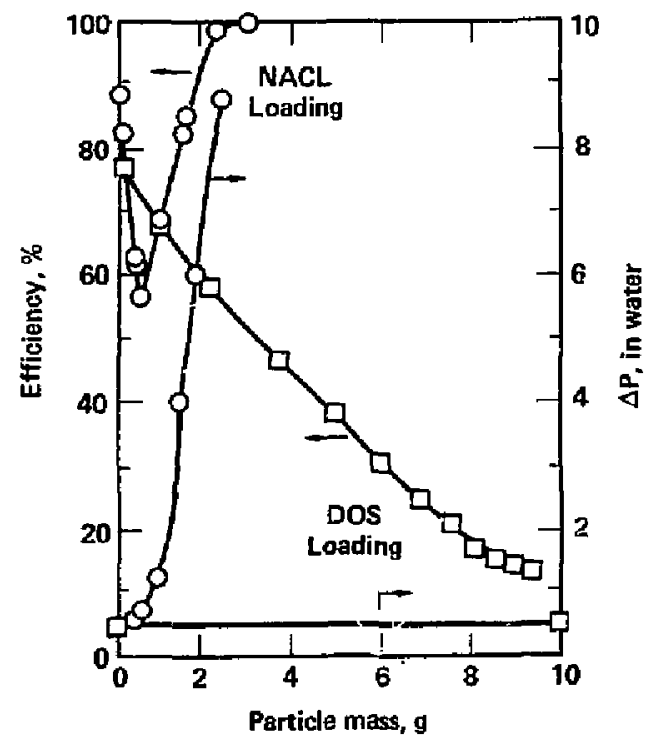

Fig. 72 Filtrete $200 \mathrm{~g} / \mathrm{m}^{2}$ performance with aerosol loading, face velocity $=64 \mathrm{~cm} / \mathrm{s}$. 
deposited particles. Note that the point at which the efficiency increases correspond: : losely with a rapid rise in pressure drop. This behavior would be typical for any solid aerosois, ramely the conversion from a capture mechanism that is primarily electrical to that of mechanical capture. The mechanical capture in this case is due primarily to the previously deposited solid aerosols rather than due to the fibrous media.

In the case of DOS-aerosol loading, we see a continual decrease in efficiency and relatively no change in pressure drop as the aerosol is loaded on the filter. The decrease in filter efficiency is due to the neutralization of fiber charge as the charged DoS aerosols deposit on the fibers. In contrast to NaCl loading, there is no minimum and subsequent increase in efficiency as the filter becomes loaded with DOS aerosols. This occurs because the liquid DOS aerosols do nat form a particle deposit that significantly increases the mechanical efficiency of the filter.

\section{Aerosol Petetration as a Function of Particle Diameter}

In our evaluation, we also measured aerosol penetration as a function of particle diameter at each stage of filter loading for NaCl and DOS aerosols. The penetration as a function of particle diameter for $\mathrm{NaCl}$ aerosols is shown in Fig. 73 where the penetration curve for an initially clean filter is compared with three additionai penetration curves corresponding to increasing deposits of $\mathrm{NaCl}$ on the filter media. The penetration at all particles sizes initially increases with loading up to 0.5 grams and then decreases with further aerosol deposition. Also, the point of maximum penetration has shifted to smaller particle diameters with increasing particle loading. The particle -ize of maximum penetration has shifted to smaller sizes with increasing particle loading because the dominant collection mechanism has shifted from electrical capture to mechanical capture by the particle deposits that develop on the fibers.

Increasing loadings of DOS aerosols and aerosol penetration is shown in Fig. 74. The penetration is at zero particle-mass loading, and at DOS loadings of $0,1.6$, and 3.3 grams on the filter. Figure 74 shows ar: overall increase in penetration at all particle sizes and an apparent shift in the particle diameter, with the maximum penetration occurring with larger particle diameters. The diameter of maximum penetration shifts to larger particle 


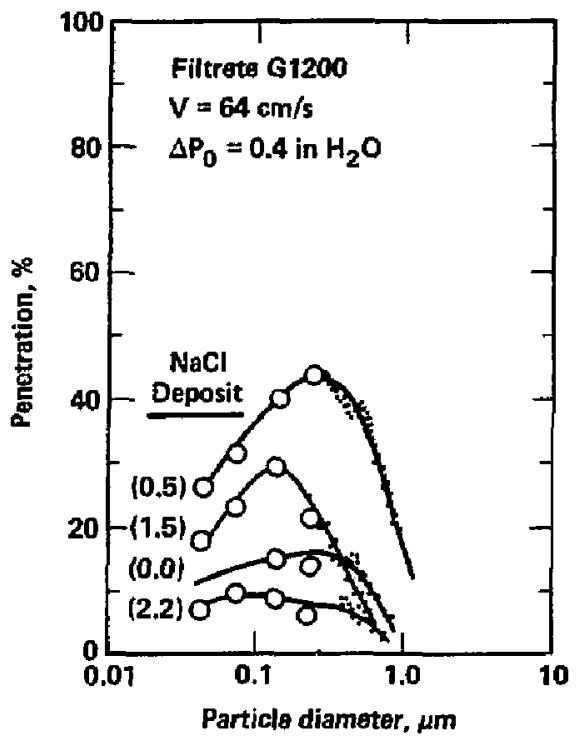

Fig. 73 Aerosol penetration at increasing mass loading grams per filter unit for $\mathrm{NaCl}$ aerosols, Filtrete $200 \mathrm{~g} / \mathrm{m}^{2}$, face velocity $=64 \mathrm{~cm} / \mathrm{s}$.

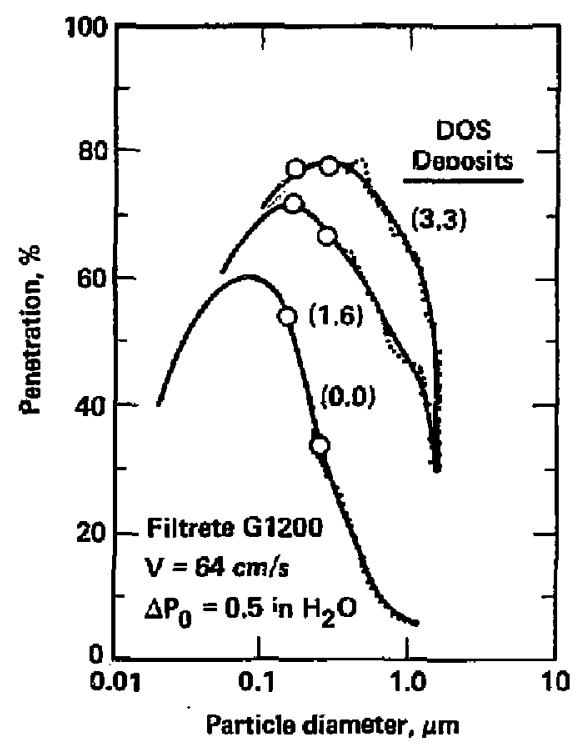

Fig. 74 Aerosol penetration at increasing mass loading grams per filter unit. for DOS aerosols, Filtrete $200 \mathrm{~g} / \mathrm{m}^{2}$, face velocity $=64 \mathrm{~cm} / \mathrm{s}$. 
diameters because of the loss of electrical attractive forces in the permanelt ly charged filters. We had previously concluded that larger particles are collected by polarization attraction while the smaller particles are collected by Coulombic attraction. For permanently charged filters, the larger particles are polarized by the electric field generated by the charged filters. Thus, for neutral aerosols or aerosols having a low charge, an electrified filter will show a higher efficiency for the larger aerosols than for the smaller aerosols. This results in a filter efficiency curve with the minimum falling at the small particle-size range. When the electrified filter is neutralized, the polarization attraction is lost and the minimum efficiency shifts to the larger particle size. In our experiments with externaliy applied electric fields, the diameter of maximum penetration shifted from smaller sizes to larger sizes when the electric air filter was neutralized. 42,51 We believe the same phenomena is responsible for the shift in peak penetration to larger particle sizes when the permanently charged filter is neutralized.

\section{Technioues for Decreasing Filter Determination}

Permanently charged filters are subject to deterioration due to actual usage in collecting both solid and fiquid aerosols, and by various environmental conditions, such as water sjlutions with trace contaminants of ions and a wecting agent, as well as exposure to organic solvents. Th is filter deterioration places severe restrictions on the applications of permanently charged filters and is the primary factor limiting its widespread use. A promising solution to this problem appears to be the application of a protectite coating around the charged fibers in an effort to prevent the charge neutralization. Several types of coatings were tested fncluding a vapor-deposited parylene, silicone polymer by dissolution, cellulose acetate butyrate by a spray technique. 52 The water immersion technique was selected as being representative of mild charge deterioriation. Although the vapor-coated parylene indicated some degree of charge loss prevention, only the front few layers of fibers within the media were coated. Other coating techniques usually resulted with fiber discharge during the application process. 
Permanently charged $f i$ ters have much higher efficiencies than uncharged $f j$ ters but they suffer from a loss of electrical enhancement oue to the filter discharging. Three mechanisms which contribute to the lass of fiber charge are (a) direct neutralization of fiber charge by charged aerosols or ions, (b) chemical reaction of organic solvents with the charges on the polymer surface which are presumably free radicals or ions, and $(c)$ concellation of the net fiber charge by the close proxinity of opposite charges. The problem of filter discharging is inherent to permanently charged filters as presently designed and will limit the widespread use of these filters. Since the filter discharging problem is tute to charged aerosols or reactive chemicals, field applications will have to avoid these agents. Thus, permanently charged filters will be ideally suited for filtering neutral or low-charged aerosois that occur in filtering atmospheric aerosols in building ventilation systems.

Filter Exposure to Water. We found that permanently charged fitters may lose some of their efficiency due to exposure to water. We measured the efficiency of the permaneitly charged filters before and after immersion in water solutians. Filters were immersed in the solutions for approximately 30-40 minutes, rinsed three times and allowed to dry. This relatively drastic test was conducted to simulate the extreme case of a saturated fiiter that coulf arise under high-humidity conditions.

We conducted a number of experiments to measure the effect of water exposure on filter efficiency. Figure 75 shows the efficiency indicated by aerosol photometer measurements before and after rinsing filters with water solutions for a number of different solutions beginning with pure water, water and acid, water and sait, water and a surfactant, and finally water and $\mathrm{NaCl}$ and a surfactant. We see, for the case of pure water, a slight deterioration of the efficlency on immersing the filter in water. We attribute this deterioration to the neutralization of charge readily accessible on the surface of the filter media. Immersing the filter in a water-nitric acid solution shows similar deterioration. We obtained dentical results with a solution of water and $\mathrm{NaCl}$.

He added the acid and $\mathrm{NaCl}$ in an effort to see if the ions would cause additional deterioration of the charge. In fact, no further deterioriation occurred due to the ions. Since these water solutions were unable to wet the 


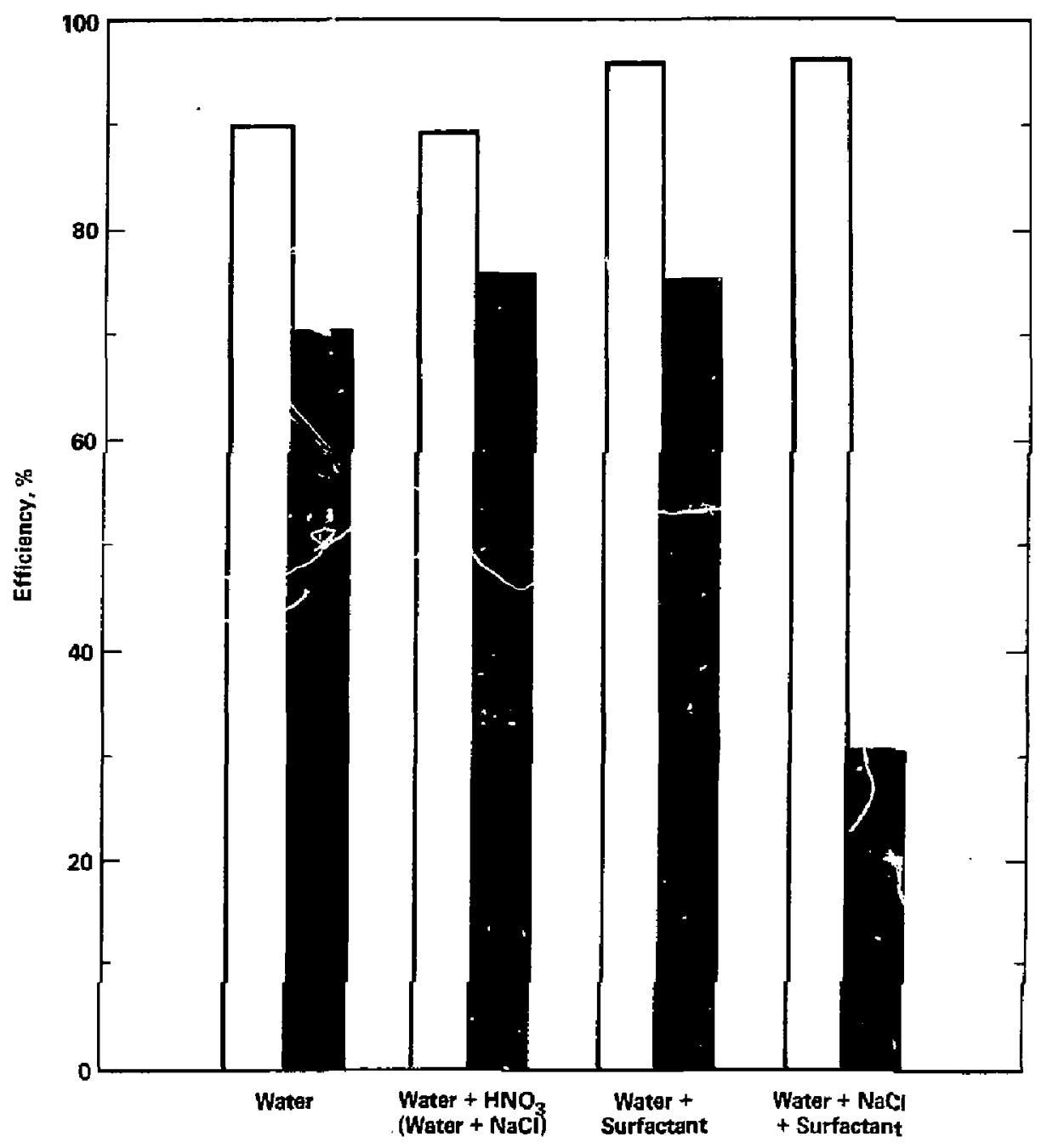

Fig. 75 Efficiency of Filtrete $200 \mathrm{~g} / \mathrm{m}^{2}$ before and after immersing in water solutions, $\mathrm{NaCl}$ aerosols, face velocity $=64 \mathrm{~cm} / \mathrm{s}$. 
fiber surface, we surmised that the solution was unable to mate physical contact with the filter surface. To test this hypothesis, we immersed the filter in a water solution having a surfactant. The results were similar to the imersion in pure water alone. In this case, we had modest deterioration in the filter efficiency after immersion. Again, we attribute this deterioriation to the fiber surface charge being readily neutralized on the filter surface. When we added $\mathrm{NaCl}$ to the water and surfactant solution, we observed a dramatic decrease in filter efficiency. Thus, the additional ions from the $\mathrm{NaCl}$ will indeed neutralize the filter charge if a surfactant is used to wet the fiber surface.

Organic-5olvent Exposure. Another adverse environment that we examined was exposure to organic solverts. This is an important consideration for applications of the permanently charged filter in respirators desizned to remove toxic particulates and gaseous matter. In Fig. 76 , we show the efficiency of the permanently charged filters before and after rinsing them in a number of arganic solvents. Again, this test is rather severe, but it is une intended to simulate extreme environments to which the filter could be exposed.

When the permanently charged filter is irmersed in alkane solvents (hexane, heptane and iso-octane), we see c relatively mild deterioration in efficiency. This is very comparable to chat seen when immersing in pure water. In contrast to the water solutions, all nf the organic solvents completely wet the filter surface. When we imerse the filter in a cyclohexane solvent, we see a greater decrease in efficiency. After immersion in a benzene or toluene solvent and a MEK/acetone solution, the filter efficiency has dropped to the leval of complete neutralization. A factor that would explain increasing deterioration with the solvents is the increasing chemical reactivity of the solvents as we go from hexane to cyclohexane to benzene, toluene and MEK/acetone. Therefore, we suspect that these solvents are able to react chemically with the surface charge (presumably present on the surface as free radicals or ions;.

since the exposure of the filter to liquid organic solvents is unlikely to occur in field applications, we examined the effect of filter exposure to organic vapors. We placed a sheet of Filtrete media, $100 \mathrm{~g} / \mathrm{m}^{2}$, in a sealed container saturated with MEK/acetone vapor for 24 hours. Filter-efficiency measurements were made on the filter before and after exposure to the organic 


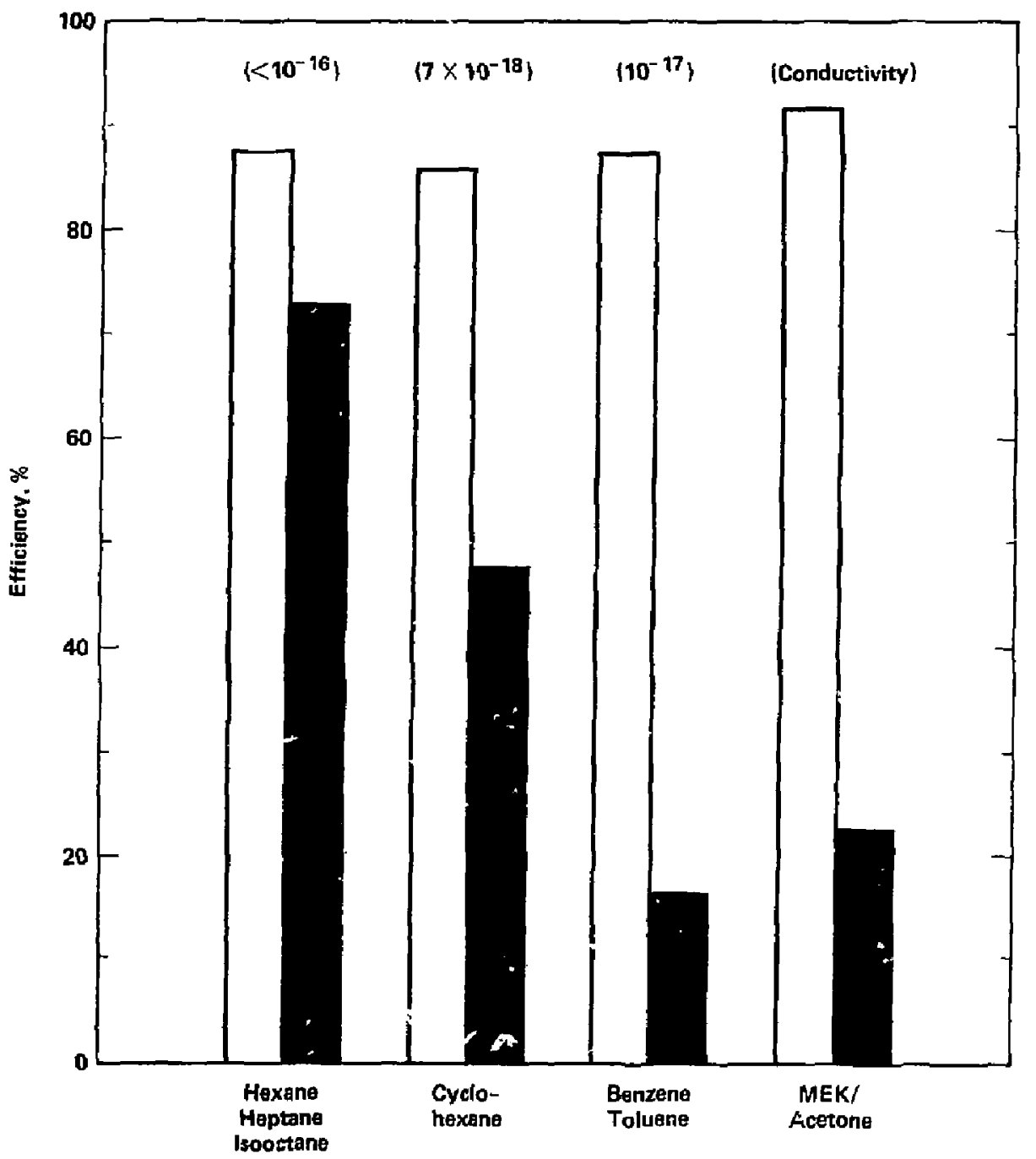

Fig. 76 Efficiency of Filtrete $200 \mathrm{~g} / \mathrm{m}^{2}$ after exposure to organic chemicals, NaCl aerosols, face velocity $=64 \mathrm{~cm} / \mathrm{s}$. 
vapors using NaCl aerosols and the light-scattering photoneter. Ho found the efficiency at a face velocity of $64 \mathrm{~cm} / \mathrm{s}$ oropped fram $71 \%$ to $49 \%$. Although the decrease in filter efficiency is less with organic vapors than with organic 1iquids, our tests demonstrate that exposure to organics is a major problem with this filter.

Anti-static spray. We have also sprayed the permanently charged filters with an anti-static agent obtained from Sprayway, Inc., Addison, Illinois. We did this in an effort to determine what fraction of the total efficiency is due to electrostatic attraction alone. Assuming that the anti-static agent is $100 \%$ effective in fleutralizing all charge on the filter, these tests would al low us to assess the degree to which the electrification is responsible for the total filter efficiency.

As shown in Fig. 77, the electrification is responsible for nearly all of the efficiency. In this case, the efficiency measured with a light-scattering photometer decreased fram $90 \%$ before spraying to $10 \%$ after spraying with the anti-static agent: Simiiar experiments with the other types of permanenisy charged filter are reported elsewhere. 52

NaCl-Aerosol Lodding on Filtrete. Figure 78 shows the comparison test results on loading the standard and neutralized Filtrete media sith $\mathrm{NaCl}$ aerosols. As seen before, the transition from a predominantly electrical collection to predominantly meshanical collection produces the minimum in the efficiency during the $\mathrm{NaCl}$ lodding for the standard Filtrete. In contrast, the neutralized Filtrete shoss a systeinatic increase in efficiency and pressure drop as $\mathrm{NaCl}$ particies load the filter. Note that the efficiency of the discharged filter approaches that of the charged filter at higher particle loadings. This is what would be expertas since the collection mechanism for both filters wi? 1 be dominaced at high-particle loadings by mecinanical capture due to the priticle Jeposits. The efficiency of the initially cirarged filter is still slightly higher than that of the initially discharged filter, which suggests the initially charged filter was not completely neutralized by the $\mathrm{NaCl}$ aerosols. Within the loading region where elecirical attraction is still significant (below 1.0 grams of NaCl), tha charged filter has a measumeable loading entancenent over the discharnaj filter. We should also hentisil tiat overcharged aerosols will demonstrate increased particle loading bec: . the charged filter will not be neutralized as rapidly. 


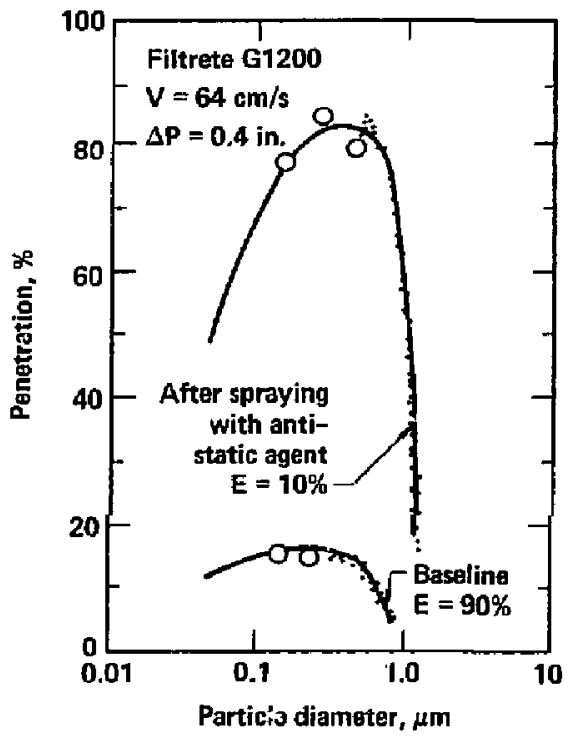

Fig. 77 Aerosol penetration of Filtrete $200 \mathrm{~g} / \mathrm{m}^{2}$ before and after antistatic spraying, $\mathrm{NaCl}, U=64 \mathrm{~cm} / \mathrm{s}$.

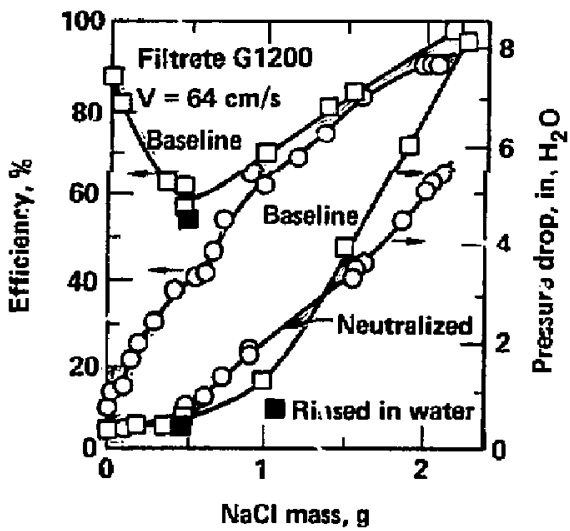

$\mathrm{Fig} .78, \mathrm{Fi}$ ter efficiency and pressure drop of rharge and discharged filtrete $200 \mathrm{~g} / \mathrm{m}^{2}$ during loading with $\mathrm{NaCl}$ aerosols, $\mathrm{V}=64 \mathrm{~cm} / \mathrm{s}$. 
After the initially charged filters had been completely loaded with $\mathrm{NaCl}$ aerosols, we added on an experiment in which we rinsed the filters in water in an effort to remove the salt deposits. We were able to remove all of the salt deposits by this method except for $61.5 \mathrm{~g} \mathrm{NaCl}$. Measuring the efficiency and pressure drop after this rinsing revaled that ve had effectively reached the same values that were originally obtained at that particle mass loading. The data, shown in Fig. 78 as solid squares, illustrate that once the dominant mechanism for particle capture of solid aerosols is controlled by the mechanical mechanism, we no longer have a continued deterioration of the Filter charge. Beginning at the point of minimum efficiency, the filter will ito longer lose additional fiber charge because the newly collected particles will be trapped by previously deposited partictes, and will be unable to neutralize the fiber charge.

\section{EFFECT OF PARTICLE LOADING ON FILTER EFFICIENCY}

We have addressed previously efficiency enhancement of electric air filters. Here, we review the aspects of another enhancement--that of lower filter resistance at comparable particle-mass loadings. We know that for solid particles, filter loading, or elogging, increases the collection efficiency of a filter at the expense of increasing the pressure drop. Despite the fact that most filters are operating at various levels of particle loading, the clogging process is difficult to understand because the formation of particle deposits is highly nonlinear, depending upon previous deposits and all the aspects describing clean filters affecting filtration. We developed f llter-loading models based on a phenomenological approach that avoids the determination of the detailed capture mechanisms and flow streamines in a loaded filter. These models wi!l be developed after our loading studies are presented.

\section{Experimental Results}

For the filter-loading experiments described here, we again used the AF-series glass-fiber media available from Mansville. We used the solid, $\mathrm{NaCl}$ aerosol for test aerosols in all rases. 
The effect of particle $:$ ading on the collection efficiency and pressure drop of two layers of AF-18 media is shown in Fig. 79. No electric field was applied. The two sets of curves correspond to two face velocities, 12 and $66 \mathrm{~cm} / \mathrm{s}$. This data was obtained using the flame photometer with a reduced particle concentration. Both filters experienced a large increase in collection efficiency due to mass loading. The more efficient filter, at $66 \mathrm{~cm} / \mathrm{s}$, is the first to $\mathrm{clog}$, a: seen by tie sharp increase on pressure drop at 2-gms loadings.

The cther relationship betwoen particle size and filter clogging is shown in Fig. 80 where the filter efficiency is plotted as a function of particle size at increasing stages of clogging. The parameter values adjacent to each of the efficiency curves represent the mass \& Nac? trapped on the filter. The curve with zero particle mass represents the efficiency curve of a clean filter. At increasing levels of clogging, the efficiency increases for alt particle sizes and the point of minimu-filter efficiency shifts to shaller particle sizes.

It is interesting to compare the results with a clean fijter having a smaller fiber diameter. In Fig. 81 , we show three graphs of collection efficiency plotted as a function of particle size. The two solid curves were taken from Fig. 80, one representing a clean AF-18 filter (median fiber diameter of $3.5 \mathrm{um}$ ) and the other representing the same filter after being clogged with $5.7 \mathrm{~g}$ of $\mathrm{NaCl}$ aerosols. The number median diameter of the aerosols was $0.28 \mu \mathrm{m}$. The dashed curve represents the efficiency of a clean A.f -4 filter which has a median fiber diameter of 0.73 um. Note that this filter has nearly the same efficiency as the filter with a large fiber size clogged with small-diameter particles. The particle deposits during the collection process have effectively transformed the large-diameter fibers into smal1-diameter tibers. As we will see later, this supports our theoretica? assumptions that particle deposits can be treated as newly formed fibers.

Eiectrified Fibrous Filter. The data presented in Fiy. 82 illustrate the effects of loading on an electrified fibrous filter. The two sets of curves are the pressure loss and collection efficiency for the filtar with no tlectric field and with a fie?d of $10 \mathrm{kV} / \mathrm{cm}$. The difference in the initial pressure drops of the two filters is not due to the applied electric field, sut rather to the variations in filter construction. The two primary effects of electrification, enhanced filter efficiency and service life, can be readily seen. 


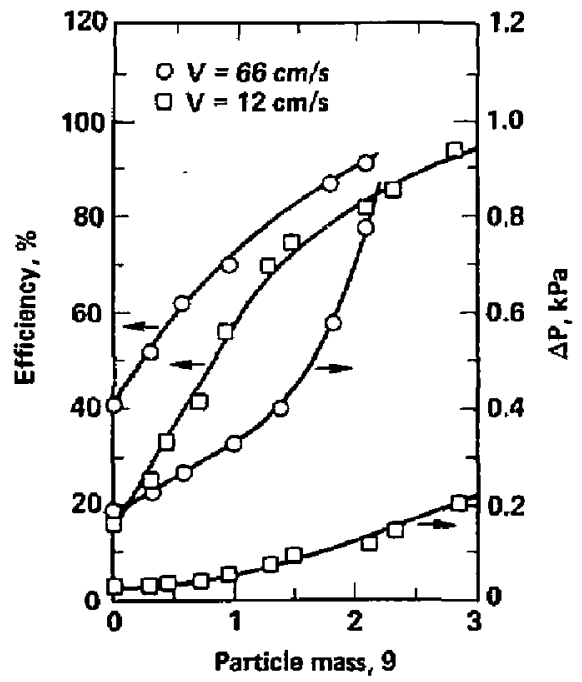

Fig. 79 Filter efficiency and pressure drop across AF-18 fi\}ters during filter clogging at different face velocities.

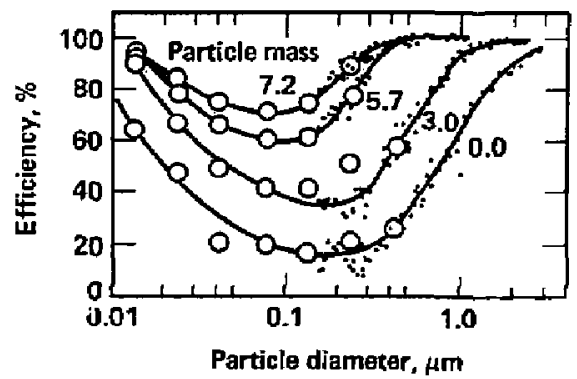

Fig. 80 Filter efficiency as a function of particle size at increasing stages of clogging. 


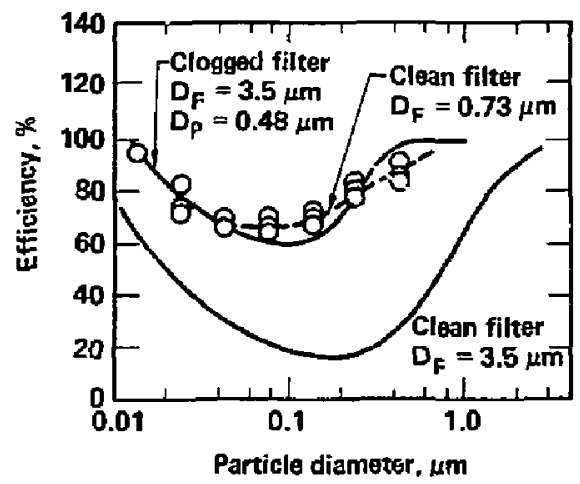

Fig. 81 Comparison of filter efficiencies as a function of particle size for a clean AF-4 filter with a clean and clogged AF-18 filter.

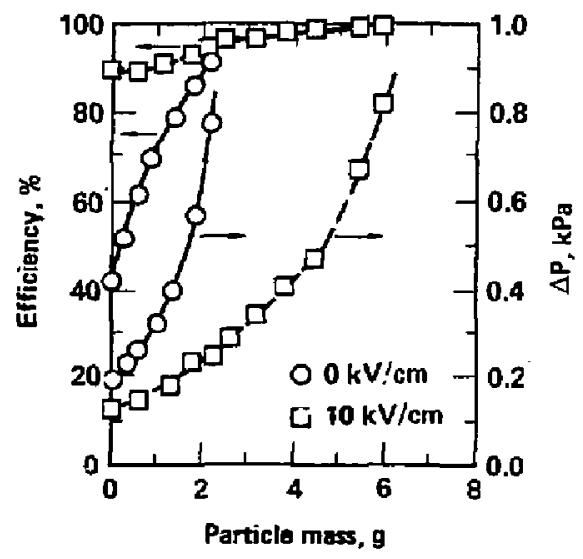

Fig. B2 Experimental measurements of filter efficiency and pressure drop for two similar filters operating with and without an electric field during filter clogging. 
The service life or a filter is generally defined in terms of the time or particle-loading required to reach a certain pressure drop. If the service life is defined in terms of the particle mass required to reach $0.8 \mathrm{kPa}$, then the electric field has increased the filter serwice life from $2.2 \mathrm{~g}$ to $6.0 \mathrm{~g}$. Tive electric field has also increased the average service life efficiency for the same filters from $70 \%$ to $95 \%$. For clean filters (zero particle mass), the efficiency increases from $40 \%$ to $90 \%$ when $10 \mathrm{kV} / \mathrm{cm}$ is applied. According to our results, filter clogging increases the efficiency for the filter without an electric field much faster than for the filter with an electric field. One may get the wrong impression that effect of the electric fiold is diminished as the filter becomes clogged. This is not correct since the ratio of penetration with and without the electric field, $P / P_{0}$, is constant as the particle mass increases. The pressure drop corresponding to the two penetration values musi be constant when caiculating the penetration ratio.

\section{Morphology of Particle Deposits}

The marphology of the particle deposits in clogged filters is the most important factor cortrolling the increase in filter efficiency and pressure drop. Knowing how the deposits initially form and change at increasing particle loadings and under different conditions is important for understanding the experimental abservations of clogged filters. Payatakes has characterized filter-clogging as a sequential process in which the morphology of particle deposits goes through four major stages 53 :

(1) Particles deposit directly an the filter fibers; deposit morphology is characterized by isolated particles randomly covering the fiber surface.

(2) Particles deposit preferentially onto previously deposited particles as well as directly onto the fiber surface. Deposit morphology is characterized by extensive surface coverage of particle dendrites.

(3) Particles deposit almost exclusively on particle dendrites. Deposit morphology is characterized by particle dendrites intermeshing with their neighbors, forming a coat of particles nonuniform thickness around each fiber.

(4) Particles deposit exclusively on the intermeshing particie dendrites. Deposit morphology is characterized by particle deposits bridging the gap between neighboring fibers. 
Stages 1-3 have been observed in laboratory experiments with monodisperse polystyrene and methylene blue spheres deposited on individual

fibers. $(54,55,56)$ payatakes recognized that there is an extensive over lap between the various stages, not only with individual fibers, but also through to the depth of a fibrous filter. ${ }^{53}$ In many cases, fibers deep within the filter are at Stage 1 while the fibers close to the front surface are at Stage 4.

We took a series of electron micrugraphs of the particle deposits at increasing levels of filter-clogging to determine the morphology of the depousits with heterodisperse aerosols and real filters. Previous studies were conducted with monodisperse spheres deposited on a single fiber. Although these more basic experimentr are easier to interpret, we wanted to establish the morphology of the deposits under real conditions. He feit that the non-ideal struccure of real filters may either alter the deposit morpholagy or introduce additional variables to the deposit morphology observed in single-fiber studies. The purpose of these $c$ icron nicrographs was to qualitatively describe the morphology of particle deposits for use in constructing realistic madels of filter-r.logging.

Figure 83 shows a series of electron micrographs of ilacl deposits at. increasing level: of filter-clogging on the front surface of AF-4 filters. First stage of filter-clogging in which captured particles form a randon covering over the fiber surface is seen in (A). At increasing particle loading, the deposit then covers the fiber surface more or less uniforinly as shown in $(B)$. Once the fiber surface is sufficiently covered, incoming particles are captured on the previous deposits to form particle dendrites (C) and (D). As more particles deposit on the filter, the particle dendrites on each fiber intermesh and finally bridge the gap between neighboring fibers. These trends are seen in (E) and (F). In general, the morphology of the particle defosits at various stages of filter clogging shown in these micrographs agree with previous studies on single fibers. ${ }^{54-56}$

Our studies show an extensive range of particle-deposit morphologies coexisting within the same filter. Figure 83 (B) and (C) are electron micrographs of different regions on the same filter. The deposits in (B) are in Stage 1 of filter-clogging, while the deposits in (C) are in Stage 2.

Particle deposits at increasing magnification of another megion within the same filter are shown in Fig. 84. These electron micrographs indicate that the particle deposits are in Stages 3 and 4 of filter-clogging. At the outer 

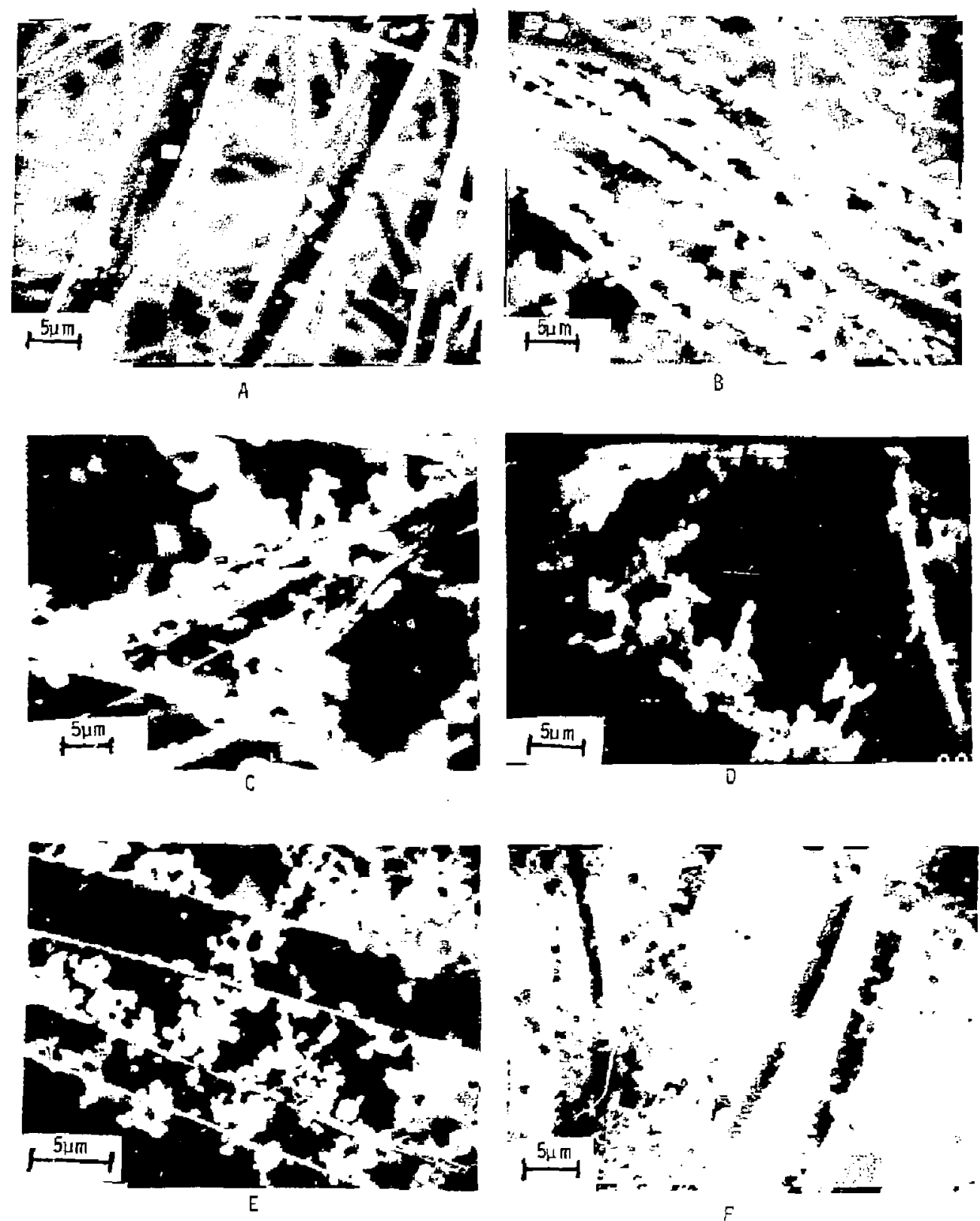

Fig. 83 Electron micrographs of particle deposits showing different stages of filter clogging on AF-4 filters. 


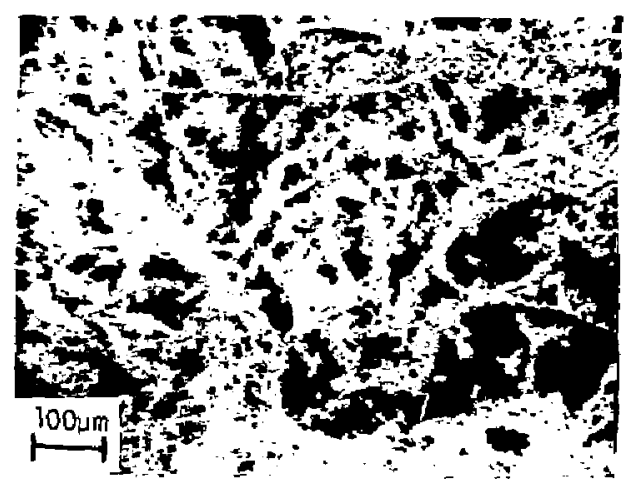

A.

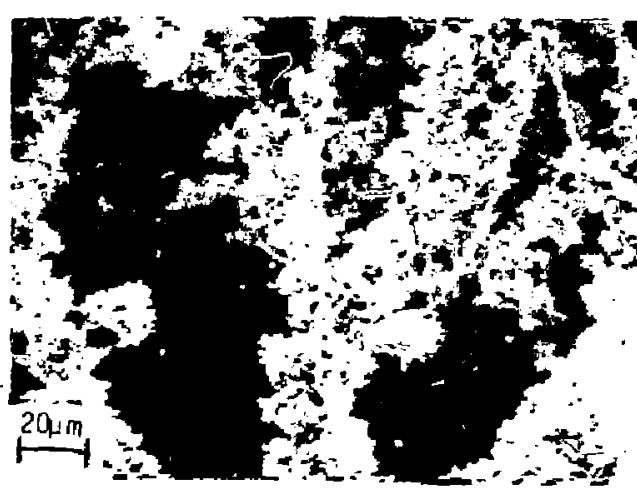

8

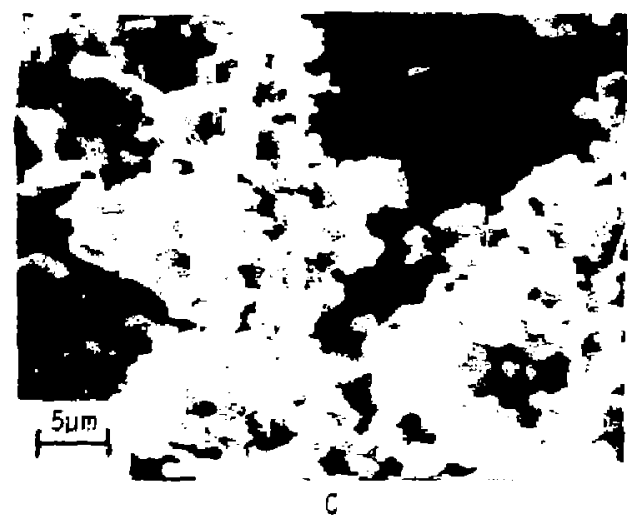

Fig. 84 Electron micrographs of particle deposit's at increasing magnifications for a clogged AF-4 filter. 
edges of (A), the particle deposits have extensively bridged between neighboring fibers to the point where individual fibers are hardly visible. However, in the central region of the micrograph, the particle deposits farm a more or less uniform coating over individual fibers. Increasing the magnification within this region shows a very densely packed deposit that differs considerably from the more open structure seen in $(E)$. We believe that the advanced stage of filter-clogging in (A) (C) is due to the increased flow resulting from a less restrictive region of the filter. Davies has described such preferential clogging in terms of plugging up holes in low-quality filters.

The formation of a uniform coating around individual fibers or the bridging between neighboring fibers is determined by the presence or absence of a nearby fiber. The deposits in Fig. 83(E) had formed a more or less uniform coating around each fiber before bridging the gap between the two fibers. A final illustration of the variation in deposit morphology within a given fiter is shown in Fig. 85. Although most of the deposits are in Stages 2 or 3 of filter-clogging, regions of Stage 1 and 4 particle deposits can be found in regions of the filter.

Dne of the primary reasons for our interest in the morphology of particle deposits was to understand the mechanism responsible for the lower pressuredrop increase that occurs during filter-clogging when an electric field is applied across the filter. To investigate this phenomenon, we made a series of electron micrographs of filters that had been clogged either with or without a superimposed electric field (Fig. 86). Figure o6 (A)-(C) represents increasing magnifications of the particle deposits on the surface of an AF-18 filter with no electric field applied during filter-clogging. The comparable case with an applied electric field is shown in $(D)-\langle F)$. In these tests, we used $A F-18$ filters, which have a median fiber diameter of $3.5 \mu \mathrm{m}$ and $\sigma_{g}=1.7$.

A comparison of Fig. 86 (A)-(C) with those in $(O)-(F)$ show that the primary effect of the electric field is to increase the particle deposition on the front surface of the filter. In $(A)-(C)$ with no electric field, a heavy deposit of particles is formed below the top layer of fibers. The fibers on the surface have only a very light particle deposit that is characterized by filters in Stages 1 and 2 of filter cloggin. With an electric field (O)-(F), the major particle deposits occur on the surface fibers of the filter. Since the packing density of the fibers is considerably smaller on the surface than 


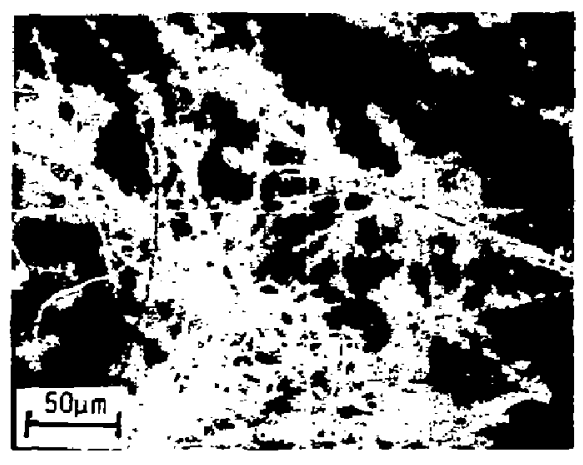

A

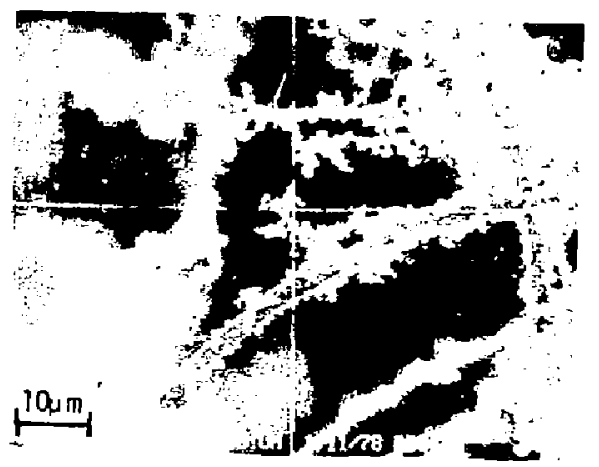

B

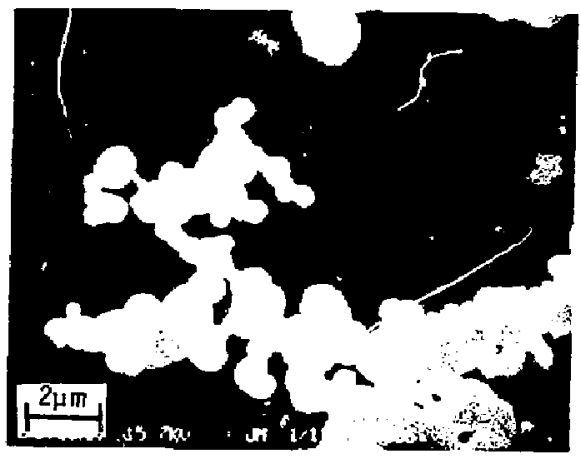

C

Fig. 85 Electron micrograph of a clogged AF-4 filter illustrating the bridging of particles across neighboring $f$ ibers. 

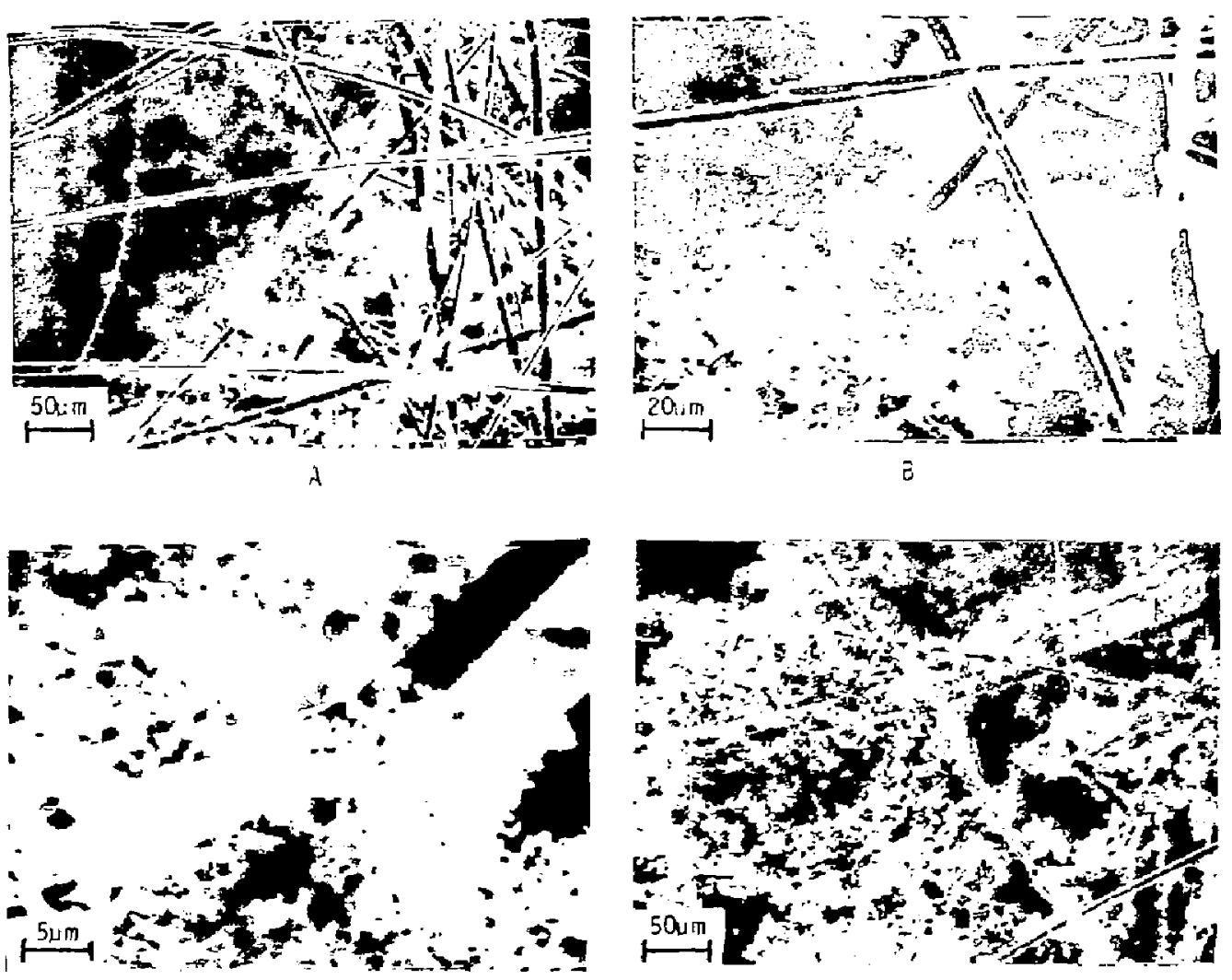

$c$

0
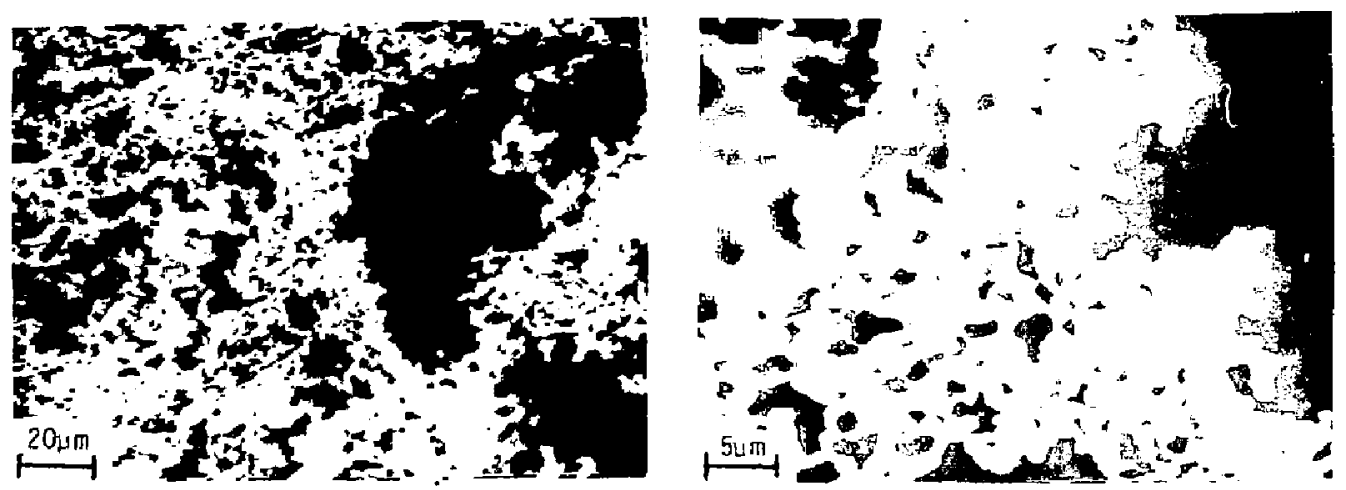

E

Fig. 86 Electron micrographs of particle deposits at increasing magnifications for clogged AF-18 filters operated $(A-C)$ with no electric field ano $(D-F)$ with an electric field. 
within the filter interior, particle deposits on surface fibers will be less restrictive than those formed within the filter interior. This less mestrictive depos. " In the filter surface is partially responsible for the decreased pressure drop that occurs in filter-clogging with di applied electric field. These observations have been previously reported by lamb et a). 57

In addition to the above differences, our fundamental studies revealeci that the morphology of the particle deposit formed in the presence of an electric field appeared to form a more uniform and densely packed deposit than the sase without an electric fieid. 58 This observation applies only for the early stages of filter-clogging. At the higher siages of filter-clogging. there appeared to be little difference in the deposit norphologies with or without an electric field.

An important aspect of the particle deposit morphology that we are $u^{r}$ able to examine is the deposit on the downstream side of the filter fibers. Particles cannot deposit on the downstrean side of the fibers with only mechanical forces. With electrical forces, there can se a significant deposit. These findings have bean substantiated in other theoretical 58,59 and experimenta $1^{60,61}$ studies. Understanding the particle deposition on the downstream side of the fibers is important because of the dramatic increase in the avalable filtering area and the increased service fife that results from particle-loading on both sides of the fiber surface.

We can propose two explanations for the decreased pressure drop at the same particle-mass loading that is observed for filters operating with an electric field: (i) a macroscopic rhange where the electric field shifts the particle deposits from the interior, more dense region of the filter to the more open region on the surface, and (2) a microscopic change in the deposit around individual fibers. In the early stages of filter-clogging, the electric field causes a uniform and der'.e deposit over the fiber surface than that which occurs without an electric field. However, at the later stages of filter-closging, the morphology of the deposits do not appear to differ significantly with or without an electric field. The electric field, therefore, has the net effect of extending the duration of the early stages of filter clogging. 
Our simplified theoretical models predict the behavior of filter-clogging with and without an applied electric field. The models are based on the geometry of particle deposits and avoid the problem of detaiing the capture mechanisms of particles in a loaded filter.

As we have seen from the scanning electron micrographs in Figs. 83-86, co*lected particles form dendrites that are highly irregular branched and unbranched chains of particles protruding from the fiber surface. Deposits have a complex geometry that varies considerably over the fi?ter fibers and during the clogging process. Becauss of these complexities, we developed and constructed idealized deposit geometries (Fig. 87).

We show three different geometries of particle deposits, each of wiich with a different function for the cross-section area. Each of these geometries yieids a different set of rquations for pressure trop and efficiency. The dendrite model is characterized by a series of particle chains, i.e., denorites, extending from the fiber surface. The increasing fiber model represents a geometry where the particles are more or less closely Dacked around the fiber and have the effect of increasing the fiber radius. (We would expect that the initial particle deposits on a clean fiber, with or without an electric field, could be approximated by this model.) The increasing fiber model and the dendrite model represent parts of a more realistic model, the combined model.

The combined mode] can oe viewed as a combination of dendrite and increasing fiber models, the proportion of the two models changing with different stages of filter-clogging. In the beginning (Stage 1), particie deposits on a clean fiber can be characterized in terms of the increasing fiber model. Once a sufficient number of particles has deposited, particle dendrites form (Stage 2) and the deposit begins to resemble the dendrite model. At increasing levels of clogging, neighboring dendrites intermesh (Stage 3 ) and the deposit once again resembles the increasing fiber model. These models break down at the final stage of filter-clogging, where particle deposits bridge the gap between neighboring fibers, and form an internal sake (Stage i).

We will give mathematical descriptions of the two models. A mathematical description of the last stage of clogging is (combined model) not as important 

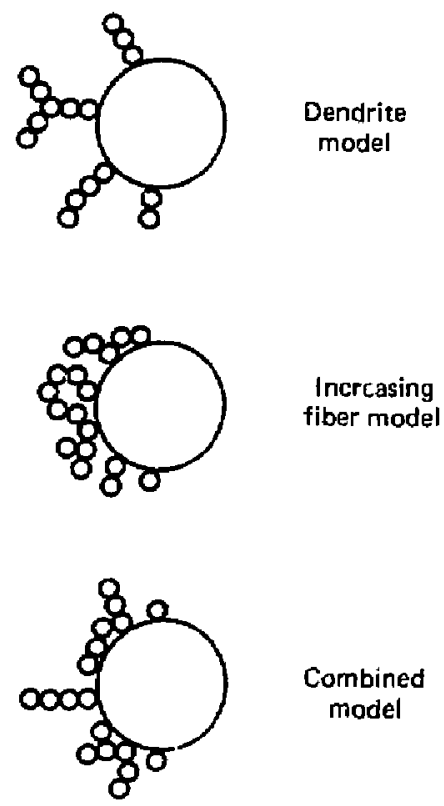

Air flow

Fig. 87 Schematic of the particle deposits used in the three filter clogging models. 
as for the earlier stages because a filter is usally thrown away or cleaned when the last atage is reashed.

The Dendrite Model. We assume that the formation of particle dendrites can be appruximated as newly formed fibers. The assumed equivalence of particle dendrites with filter fibers may appear to be questionable because they are connected to the fibers and form "hairy" fibers rather than discrete fibers. Moreover, the air-flow field around a de: rite is perturbed by the flow field around the fiber to which the dendrite is attaclied. Theoretical filter models used to predict filter efficiency and resistance in clean filters are equally questionable on the same grounds. Examination of comercially available fibrous filters shows that the fibers are not well-ordered, as assumed in the theory, but are spaced nonuniformly and are often clumped together and interwoven in a complex maze. The error in using flow-cell models to predict the flow around filter fibers and around particie dendrites should not differ significantly.

The derivations rely on two parameters that are analogous to the filter fibers: the single-fiber efficiency and the fiber radius of the dendrite. An. important aspect of the derivation is the compounding effect of particle depcsit on filter efficiency, and, hence, on the rate of dendrite growth. We begin trie: -rivation of the filter penetration by considuring the fraction of particles lost, - $\mathrm{dN} / \mathrm{H}$, because of collisions with fibers and particle dendrites in a differential filter element of thickness, $d x$ :

$-\frac{d N}{N}=\frac{v}{V A}\left(d a_{F}+d a_{p}\right)$

where $d a_{F}$ and $d a_{p}$ represent the effective differential areas or the fibers and particle dendrites, respectively. Substituting expressions for $\mathrm{da}_{F}$ and dap, yields

$-\frac{\mathrm{dN}}{\mathrm{N}}=\frac{\mathrm{V}}{\mathrm{VA}}\left(\frac{\eta_{\mathrm{F}} 2 \mathrm{~A} \alpha}{\pi^{\mathrm{R}}} \mathrm{dx}+\eta_{\mathrm{P}} \pi \mathrm{r}^{2} \mathrm{dM}\right) \frac{\mathrm{V}:}{x}$ 
where $n_{F}$ and $n_{p}$ represent the single fiber efficiencies for the fibers and dendrites, respectively, and $d M$ represents tha cumulative number of particles in the differential filter element. After integrating Eq. 39 over the filter thi-kness, we have

$P=P_{0} \exp -\left(\frac{\pi V t r^{2} N_{0} \eta_{p}}{2}\right)$

where $P_{0}$ and $P$ are the penetration at time 0 and $t$, respectively, and $N_{0}$ is the concentration of particles entering the filter. Since most experimental data shows filter penetration as a function of particle mass, we can derive the corresponding theoretical equation by using Eq. 40 in a particle-mass balance. Since rate of particle-mass accumulation in the filter equals the rate of particle mass entering the filter multiplied by the filter efficiency, we can derive this expression:

$m=\left(\frac{8 \rho \mathrm{rA}}{3 \eta_{\mathrm{p}}}\right)\left[-\ln \left(\mathrm{P} / \mathrm{P}_{0}\right)+\mathrm{P}-\mathrm{P}_{0}\right]$

where $m$ is the particle mass in the filier. With Eqs. 40 and 41 , the increase in filter penetration due to dendrite growth to be calculated as a function of either time or particle mass, respectively.

Theoretical equations can also be derived for explaining the increase in filter resistance due to particle dendrites. Since we coisider dendrites to act like filter fibers, we can use the same equations to calculate the resistance of the original fibers as we could for the resistance of the particle dendrites. For a clean filter, the resistance or pressure drop is determined by

$\Delta P=\mu V r_{F} L_{F}$

where $\Delta P$ is the pressure drop, $\mu$ is the fluid viscosity, $V$ is the face velocity, $F_{F}$ is the dimensionless drag over the fiber, and $L_{F}$ is tine total length of the fiber. If particle dendrites are added to the system, the total 
pressure drop is

$\left.د P=\mu V_{(}^{\prime} F_{F} L_{F}+F_{p} L_{p}\right)$

where $F_{p}$ and $L_{p}$ are the corresponding dendrite variables of dimensionless drag and total length, respectively. The dimensionless drag, $F_{p}$, is a function of the volume fraction, $\alpha$, of the fibers within the filter. M. important consideration in the pressure-drop derivation is the valuf of a used to calculate the dimensionless drag in Eq. 43.

We included an additional pressure drop of the interference effect in our model to avoid the assumption that pressure drop due to the fibers and dendrites are independent. We increased the fiber and dendrite volume fractions by factors $L / L_{F}$ and $L / L_{p}{ }^{*} L$ is the total length of both fibers and dendrites. The dimensionless drag is then computed with the increased volume fraction and represents the drag from a hypothetical filter containing either all fibers or all dendrites. These drags are given by

$F_{F}=F_{F}\left(\frac{\alpha_{F} L}{L_{F}}\right)$ and

$F_{p}=F_{p}\left(\frac{\alpha_{p} L}{L_{p}}\right)$

The total length of the fibers or the dendrites in these hypothetical fitters equal the combined leingth of the fibers plus dendrites in the originat filter. We have assumed implicitily that the air flow around each fiber or dendrite is independent of the size of the neighboring fibers or dendrites.

The pressure drop across the clogged filter can be determined once we have selected the appropriate functions for the dimensionless drag. Any of severat flow models can la used. We chose the Davies empirical equation ${ }^{62}$ because of its simplicity. For very loose fibrous filters, Davies' equation for the dimensionless drag is

$F_{i}=16 \pi \alpha_{i}^{1 / 2}$ 
where the $i$ subscript represents the fiber or dendrites. Using the definitions for $L_{x}$ and $L_{p}$ and substituting Eqs. 44-46 into Eq. 43, the following expression for pressure loss can be derived:

$\Delta P=\Delta P_{0}\left(1+\frac{R \alpha_{\mathrm{p}}}{T \alpha_{F}}\right)\left(1+\frac{R^{2} \alpha_{\mathrm{P}}}{r^{2} \alpha_{F}}\right)^{1 / 2}$

where $\Delta P_{0}$ is the pressure drop across the clean filter.

Although Eq. 47 was derived for a filter consisting of fibers and particle dendrites, it can also be used for a filter having two discrete fiber sizes. We have generalized the above derivation for a distribution of fiber sizes and reported the results in. 39

To test the accuracy of our theoretical equaticns, we compared experimental test results at different flow rates and different particle sizes to theoretical predictions. Figure 79 showed the efficiency and pressure drop plotted as a function of the particle mass trapped in the filter. The primary difference between the two experiments is the air-flow velocity used to load the filters. We replotted the two sets of data using terms from Eqs. 41 and 47 to eliminate the velocity dependence. If $\Psi$ represents the term $-\ln \left(P / P_{0}\right)+P-P_{0}$ in $E q .41$, then penetration data plotted as $\$$ vs $m$ should fall on the same curve for any filter media and experimental conditions, provided aerosol properties remain constant. Figure 88 shows the two efficiency curves from Fig. 79 replotted as $Y$ vs $m$. We multiplied the $q$ values of the two experiments with a factor $N$ to account for changes due to the velocity in the particie-dendrite collection efficiency, " $p^{\text {. The }}$ excellent agreement between the nomaiized $\$$ values seen in Fig. 88 indicates the accuracy of Eq. 41 for predicting filter-penetration values at different mass-loadings.

He made a simiar analysis of the pressure drop for the two experiments shown in Fig. 79 with Eq. 47. Since the pressure drop is linearly dependent on the velocity, the ratio $\Delta P / V$ or $\Delta P / \Delta P_{0}$ would then be independent of velocity. We platted the data as $\left(\Delta P-\Delta P_{0}\right) / V$ so the resulting curve would pass through zero at zero particle mass-loading. The resulting dat $\bar{i}$ show good agreement between the two experiments (Fig. 89). An even better fit could be obtained by nomalizing both sets of data to the same value of filter 


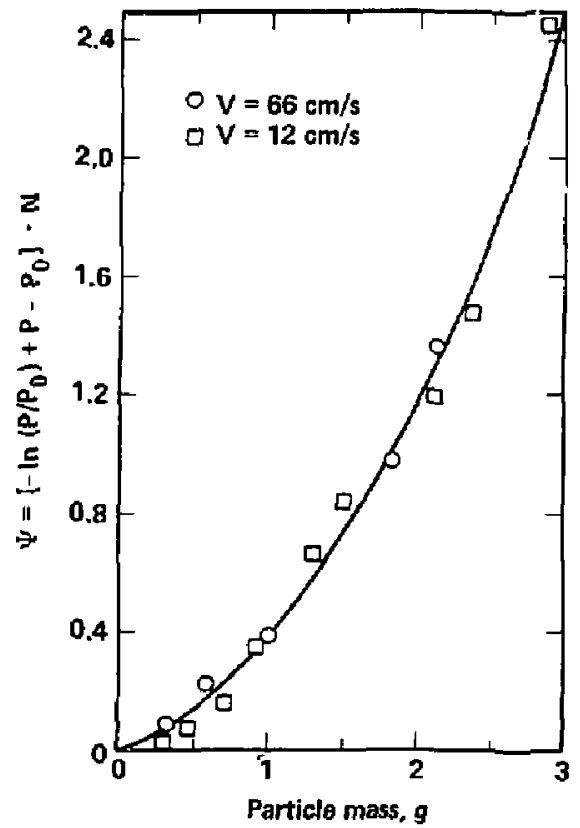

Fig. 88 Normalized penetration $\Psi$ as a function of particle mass loading for filter elogging tests at diffurent flow velocities. 


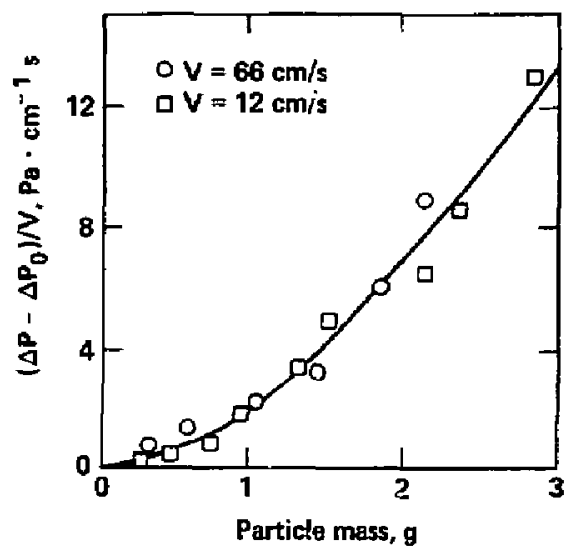

Fig. 89 Pressure drop increase per unit flow velocity as a function of particle-loading at different flow velocities. 
thicknass $(x)$ and fiber volume fraction $\left(a_{F}\right)$; however, the normalization is complicated by the compression of the filter at higher flaw rates. This compression increases the value of $\Delta P / V$ by decreasing the filter thickness and increasing the fiber-volume fraction. Note, the velocity does not appear in the terms attributed to the particle dendrites in $\mathrm{Eq} .47$. This implies that the dendrite structure is independent of the air velocity. Billings 53 found the dendrite structure did not change significantly in experiments conducted at varying air flows.

The Increasing Fiber Model. The increasing fiber model assumes that particles form a deposit that increase the fiber size withaut changing the fiber's shape. This requirement can be significantly relaxed orly and produce a minor change in the model equations. The primary requirement of th : model is that the particles form a relatively close-packed deposit on the iber surface. We have already described how the required close-packed structure is produced by the electrical filtration forces.

The relationship between the increasing fiber size and the amount of particle deposit can ve determined for the deposit geometry illustrated in Fig. 87. If we assume the particle deposit has a uni.orm thickness on the front half of the fiber and each particle occupi: a cubic cell of volume $8 r^{3}$ (47.6\% open space), then we obtain the following expression is obtained for the increase in fiber radius, R:

$\mathbf{R}=\mathbf{R}_{0}\left(1+\frac{12 \alpha_{p}}{\pi \alpha_{F}}\right)^{1 / 2}$

where $A_{0}$ is time original fiber radius, and $\alpha_{, j}$ and $\alpha_{F}$ are the volume fractions of the particles and fibers. The open spaces in the particle deposit are not inciuiod in $\alpha_{p}$.

Equation 49 represents the increase in fiber size from $R_{0}$ to $R$ due to particle accumulation on oniy one half of the fiber. The effective fiber-volume fraction ${ }^{E}$, can be derived in terms of the increased fiber size, $R$ :

$$
\alpha_{\mathrm{E}}=\frac{\mathrm{R}^{2} \alpha_{\mathrm{F}}}{\mathrm{R}_{\mathrm{F}}^{2}}
$$


Note that $a_{E}$ is not the sum of ${ }^{a} F$ and $a_{p}$.

The derivation of the penetration equation for the increasing fiber model begins the fractional decrease in particle concentration across a differential filter elemen:

$-\frac{\mathrm{dN}}{\mathrm{N}}=\frac{\mathrm{v}}{\mathrm{VA}} \mathrm{da} F$

where da $F$ is the effective differential area of thic fibers.

Substituting for the effective differential area we have

$-\frac{d N}{N}=\frac{v}{V A}\left(\frac{2 A \alpha_{F} \eta_{F} R d x}{\pi R_{j}^{2}}\right)$

We can obtain the solution of the penetration equation by substituting the increasing fiber radius, given by

$\Delta P=\Delta P_{0}\left(1+\frac{R \alpha_{F}}{r \alpha_{F}}\right)$

into Eq. $5 ?$ and integrating over the filter thickness, $X$. Unf tunately, these steps are complicated by the single-fiber efficiency, $n_{F}$, which is also a function of $R$.

In a previous report, ${ }^{42}$ we showed that the single-fiber efficiency could be grouped according to terms inversely dependent on the fiber size, $n$, and terms independent on fiber size, $\pi_{i}$, so that

$$
\begin{aligned}
& \eta_{1}=\eta_{M}=\eta_{R, P_{P}}+\eta_{1, P_{p}}+\eta_{D, Q_{q}} \text { and } \\
& \eta_{2}=\eta_{R, P_{q}}+\eta_{D, P_{q}} .
\end{aligned}
$$

Substituting these single-fiber efficiencies into Eq. 57 , integrating over the filter thickness, and simplifing the resulting penetration for the increasing fiber mode creates this expression: 
$P=P_{0} \exp -\left[\frac{16 r^{3} V t N_{0} \eta_{2}}{\left(1-\alpha_{F}\right) \pi R_{0}}\right]$

where the penetration for the initially clean filter is

$P=P_{0} \exp -\left[\frac{16 r^{3} V t N_{0} \eta_{2}}{\left(1-\alpha_{F}\right) \pi R_{0}}\right]$

The corresponding theoretical relationship for particle mass

$m=\left(\frac{\pi \rho R_{0} A}{12 \eta_{z}}\right)\left[-\ln \left(P / P_{0}\right)+P-P_{0}\right]$

Next, we derive the pressure drof equation for the increasing-fiber model. As in the dencirite model, we start with Eq. $42 \mathrm{fc}$. the pressure drop across a clean filter. When particles are closely deposited around the fiber, the total pressure drop is given by

$د P=\mu \mathrm{VFL}_{\mathrm{F}}$

where $f$ represents the dimensionless drag for the fiber and particle deposit. Since no additional fibers are added, as in the dendrite case, the fiber length, $L_{F}$, remains constant. Although several different forms of the dimensionless drag can be used in Eq. 58, we use Davies' Eq. 46, where a represents the effective fiber-volume fraction, " $E$ given by Eq. 49. Substituting Eqs. 44, 48, 49, into Eq. 58 yields

$$
\mathcal{S}=\frac{16 \mu \mathrm{VA \times} \alpha_{\mathrm{F}}{ }^{3 / 2}}{\mathrm{R}_{\mathrm{p}}^{2}}\left(1+\frac{12 \alpha_{\mathrm{p}}}{\pi \alpha_{\mathrm{F}}}\right)^{1 / 2} \text {. }
$$

or, in terms of the initial pressure drop, $\Delta P_{0}$

$$
\Delta P=\Delta P_{0}\left(1+\frac{12 \alpha_{F}}{\pi \alpha_{F}}\right)^{1 / 2}
$$


Although we have not quantitatively verified the accuracy of our theorsical expressions of penetration and resistance for the increasing-fiber model, we can compare it with the dendrite model. According to Fig. 79 , the relative penetration for the electrostatic filter decreases much slower than the penetration for the standard filter. We can corroborate this experimental trend using Eqs. 40 and 56, which represent the theoretical penetration equations for the dendrite and increasing fiber models. Taking the time derivative of the relative penetration, we have

$$
\frac{\left[\left(\frac{1}{P}\right)\left(\frac{d P}{d t}\right)\right]_{\text {den }}}{\left[\left(\frac{1}{P}\right)\left(\frac{d P}{d t}\right)\right]_{\text {inc fib }}}=\frac{\pi^{2}}{32}\left(\frac{R_{0}}{\mathrm{r}}\right)\left(\frac{\eta_{P}}{\eta_{2}}\right) \text {. }
$$

where $n_{p}$ represents all of the mechanical and electrical capture mechanisms for the particle dendrites. Since $n_{2}$ only represents a small portion of the total single-fiber efficiency, the ratio $n_{p} / n_{2}$ is very large. The ratio $R_{0} / r$ is also large. Therefore, the relative penetration changes much faster for the dendril model than for the increasing fiber model.

Comparing Eq. 59 to Eq. 47 shows that the increasing fiber model has a lower pressure drop at the same particle volume fraction than the dendrite model. In addition, the increasing fiber model has no dependence on the fibe and particle radius. The effect of different particle sizes on the pressure drop does not occur for the increasing fiber model.

We made a quantitative comparison of the dendrite and increasing fiber madels with the experimental pressure drop data shown in Fig. 70. The results are shown in Fig. 90, where we have plotted the pressue drop as a function of particle mass trapped on the fibers for similar filters with and without an electric field. As predicted by theory, the filter having the appiied electric field closely follows the pres:ure drop predicted by the increasing fiber model at low particle mass. At tigh mass loadings, the electrostatic filter deviates sharply from the increasing-fioer model and tends to follow the rate predicted for the dendrite model. Particle capture at the higher loadings is increasingly dominated by the particle deposits and less by the original fibers. At the higher particle loadings, the deposits are not constrained to follow the shape of the original fibers, and, consequently, incisase their dendritic character. 


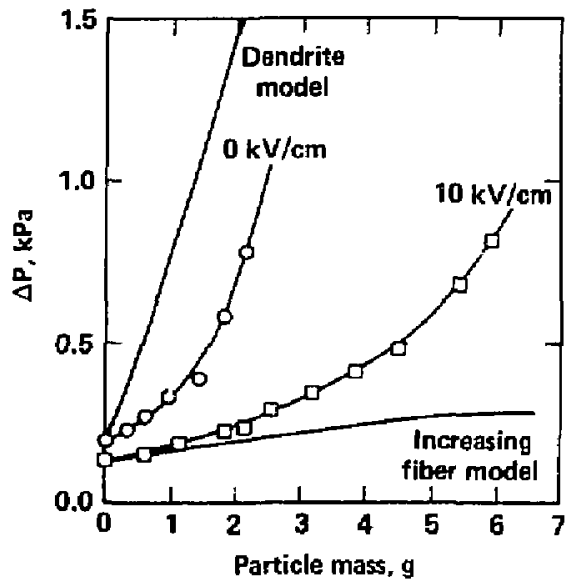

Fiy. 90 Comparison of theoretical and experimental pressure drops for filters operating with and without an electric field during filter clogging. 
A similar trend is seen for the fibers with the applied electric field. At very law particle laadings, the pressure drap increases at a rate predicted by the increasing fiber model. This trend is expected since there are no dendrites formed at very small particle-loadings. However, after a smail amount of deposit has formed, the pressure drop rapidly increases and follows the trend predicted by the dendrite madel. Comparing the two cas as with and without an applied electric field shows the primary effect of the electric field is to delay the formation of deridrites. The two experimental curves alsa show the dendrite and increasing-fiber madels are limiting cases of the more generalized model shown in Fig. 97 as the combined model. The increasing fiber model approximates the combined model at low particle loadings for the electrostatic filter and at much lower particle laadings for the standard filter. The dendrite model is the limiting case of the combined model at high particle loadings for both filters with and without an electric field. Much higher particle loadings are required for the electrastatic filter before the pressure drop increase follows the dendrice model.

DEVELOPMENT AND EVALUATION OF EXPERIMENTAL ELECTRIC AIR FILTERS

INTRODUCTION

Our extensive theoretical studies and laboratory experiments described in the previous major section were used to design criteria for estabishing parameter values of experimental electric air filters. We tased our experimental filters on the polarization method because we felt this method would be the most acceptable to the nuclear industry. The corona-charging method increases the fire hazard due to sparking. Of course, if later analyses show there is no fire risk, then the addition of a corona precharger wili significantiy improve the performance of the electric air filter. Criteria for prototypes charged only by the polarization method will differ slightly fram the criteria for experimental filters charged by the corona method, either by itself or in combination with the polarization method. The corona-charging method affects two design parameters, the high-voltage power suppiy and the high-voltage electrodes. The high-voltage power supply would require a greater output current while the :igh-voltage electrodes wou?d need to be redesigned to optimize the combined corona and polarization electrodes. 
Our design criteria for experimental electric air filters using the polarization method are summarized in Table 7 . These criteria were used for all of our filters. We shall discuss these criteria in some detail.

\section{Filter Size Limitation}

Filter size is limited by the its application and its maximum initial airflow resistance. For ventilation ducts, the $610 \times 610 \times 305 \mathrm{~mm}$ HEPA filter is generally chosen so that existing housings may be used. In other applications, no firm restriction exiscs on size although a minimum size is preferred by the industry. The limitations on air-flow resistance closely follow size restrictions in filter applications where the ventilation construction and the blawer capacity limit the total load on the ventilation system. Most nuciear facilities require that the initial pressure drop across a new filter be less than one inch of water $(250 \mathrm{~Pa})$. This pressure drop is controlled primarily by the resistance of the filter medium and the face velocity. The nuclear industry, as a whole, requires all filters to meet existing size restrictions and the pressure drop limitation.

\section{Filter-Face velocity}

After the physical dimensions, the most important design parameter is the filter-face velocity, which shouid bave a minimum yalue. A lower face velocity decreases filter resistance and increases filter efficiency and dust-holding capacity. A lower face velocity also produces a higher filter efficiency because the electrical forces have a jonger time to attract particles to the fiiter fibers. The most common technique for decreasing the face velocity is to increase the filtering area by pleating the filtering element, the number of pleats being governed by the thickness of the filtering element and the ease of replacing the filter medium.

\section{Filter Medium}

The criterion for a maximum resistance of $250 \mathrm{~Pa}$ eliminated the fabric media typically used in bag-house filters. These filters generally have 
Table 7. Design criteria for electric air filters uzing the polarization charging method.

Physical dimensions

Pressure drop

Face velocity

Fjiter medium

High-voltage electrodes

Electrodes and medium integrated or separated

High-voltage power supply

Efficiency

Dust-holding capacity

cost

Waste volume

Filter or medium replacement

Electrical safety
Limited by application, minimum value

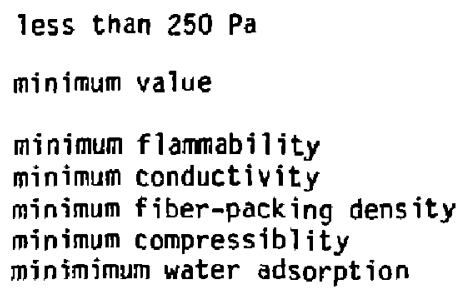

maximum open area

good structural support for filter medium good electrical contact with medium optimum electric-field orientation good insulation from ground high electrical resistance

\section{filter-change frequency}

maximum OC vottage automat ic shut down during over Toad output current below $100 \mathrm{uA}$

maximum value

maximum value

minimum value

minimum value

minimum effort

minimum hazard 
resistances that grecily exceed our criterion. We selected the deep-bed fibrous media for our electric air filters. The medium has to be nonflammable because of potential fires in the ventilation system. It needs a minimum conductil $y$ to prevent short-circuiting of the high-voltage electrodes. A medium with lower conductivity also increases the filter-collection efficiency que to the charged-fiber mechanism. Filters with high conductivity cannot maintain a large fiber charge because the charge bleeds away. Unfortunately, real materiais that are nonconductive are generally flammable. For example, glass fibers are nonilamsiable, but are moderately conductive, and plastic fibers have very low conductivities, but are flammable.

We established a minimum fiber-packing density to increase the filter-dustholding capacity. Lamb et al. have shown that electric air filtars with a low fiber-packing density have a significantly lower pressure drop during filter-clogging than filters with a high fiber-packing density. 36 Unfortunately, this lower pressure drop is obtained at the expense of decreased filter efficiency. This undesirable effect can be minimized by desighing a filter medium with a graduated fiber-packing density. Lamb et al, have shown that the fiber-packing density within most filters varies greetly; tho outer surface has a much lower density chan the incerior portion. In the absence of an electric field, particles penetrate to a region within the filter where the packing density is higher, thererure forming a heavy deposit within the fi]ter volume.

However, with an applied eleciric field, particles are captured by fibers on the front face of the filter, where more deposits can be accommodated becduse the fiber-packing density is verg low. The electric field increases fiber efficiency, which in turn is responsible for enhanced particle collection on the front face of the filter. Particle deposits on the front face of the filter form a more open structure than the deposits within the filter volume. Another property of the filter medium that is closely related to the fiberpacking density is the compressibility of the filter mat. Since compressing the filter mat increases the fiber-packing density and consequently the pressure drop, the filter media needs to have a minimum compressibility. The AF-18 medium is only moderately comp essed as compared to the AF-4 medium. There is an additional compression due to particle deposits in clogged filters. Although this additional compression is insignificant for the AF-18 medium, it is very large for the AF-4 medium. The increased ccmpresson for the AF-4 
medium is due to the smaller diameter fibers that are easier to bend. The AF- 4 filter has a median fiber diameter of 0.73 um while the AF-18

filter has a median fiber diameter of $3.5 \mathrm{um}$.

The last filter-medium requirement is minimum water adsorption. It is a well-established phenomenon that water adsorbed on an insulator dramatically increases surface conductivity. For example, the surface conductivity of soda lime glass increases from $10^{-12}$ to $10^{-7}$ mhos as the relative humidity increases from $40 \%$ to $90 x$. This increased conductivity leads to an increased current flow and eventually short circuits across the high-voltage electrodes.

We chose a glass fibrous filter for our electric air filters, based on the fiiter medium requirement discussed so far. It is nonflamable and has reasonable values for the conductivity, fiber-packing density, compressibility, and water adsorption. Moreover, these filters are inexpensive and available in large quantities. The most frequently used medium in our experimental filters was the AF-18 filter from Johns-Manville. We have already illustrated the typical performance data for this madium in terms of increased efficiency in the section on fundamental studies.

\section{High-Voltaye Electrodes}

Once we selected the proper filter medium, we determined the optimum design for the high-voltage electrodes. These electrodes require a maximum open area to minimize air resistance and to prevent masking of the filter medium, he found that the frant electrode can hide a major fraction of the filter surface, thereby accelerating the clogging process. To prevent the masking problem on the front electrode, we selected a wire-screen electrode that had over $90 \%$ of its area open. Moreover, since the front electrodes does not support the filter medium, it does not need a rigid construction. The major criterion for the rear electrode is to support the filter medium. The rear electr ode can have a relatively small open area since it cannot conceal the filter medium and adds very little resistance. He selected a perforated metal sheet with a $40 \%$ open area for our rear electrode.

One of the most important criteria for the design of the high-voltage electrodes is good electrical contact with the filter medium. If the filter mesilum does not have good electrical contact with the electrodes, the electric air filter will have the same performance as a filter with no applied voltage. 
This phenomenon occurs because the fiber charge migrates across the filter bed and it accumlulates next to the electrodes if they, sre not in contact with the medium. The accumulated charge creates an electric field within the filter medium that cancels the field produced by the high-voltage electrodes. However, if the electrades are in contact with the medium, the fiber charge that migl ates to the electrodes is neutralized and therefore cannot reduce the electric field generated by the electrodes. One of the primary causes for losing electrode contact with the medium is the compression due to air flow.

The criterion for designing the high-voltage electrode for optimum electric field orientation is not well-established. All of our studies were conducted with the electric field either parallel or anti-parallel to the air flow. No change in the filter efficiency was observed with either orientation. We chose to apply high voltage to the rear electrode in our experimental filters to minimize the possibility of deposits creating a leak path or even a shortcircuit between the high voitage and the grounded filter housing. Grounding the front electrode also provided an additional safety feature against accidentai short-circuits and electric shock hazard.

Lamb et al. performed a series of experiments on the effect of electricfield orientation on filter efficiency. ${ }^{36}$ They concladed that the electric field perpendicular to the air flow produced a much higher filter efficiency than the fieid parallel or anti-parallel to the air flow. However, in their tests, the electrodes did not contact the filter medium with the parallel and anti-parallel configuration, thcreby invalidating tise conclusions. The optimum field arientation for the maximum filter efficiency still must be determined.

The c, iterion for having electrodes with a high electrical resistance was estab.ished to minimize sparking across the electrodes. Sparking across the electrodes is undesirable because of the potential fire hazard and the decreased yoltage across the electrodes which decreases the filter efficiency. Thompson and Woodfin have shown that the use of high resistance electrodes significantly reduces the potential for sparking across the electrodes. 38 High-resistance electrodes also reduce the prodability for accidental shortcircuits and Electric shock hazards. We should point out that high-resistance electrodes do not reduce the electric field as compared to highly conductive electrodes at the same applied voltage. However, high-resistance electrodes must have a sufficient conductivity to pass the currents due to the particle charges. 
Filter Media with Integrated or Separated Electrodes

Another design parameter we considered for electric air filters was having the elecirodes be an integral part of the fijter media or be separate from the media, depending on whether the complete filter unit or only the filter medium was discarded when the medium became clogged. The frequency of filter changes in the intended application determines this. Figure 91 illustrates how the frequency of filter changes affects the total filtration cost and hence the selection of a filter design having electrodes integrated or separate from the filter media. The dasign having the electrodes integrated with the filter media has a Jow fixed cost due to the high-voltage power supply but a high operating cost since the entire filter with electrodes has to be replaced. The design having the electrodes separate from the filter media has a high fixed cost due to the increased hardware of the electrodes and housing but a low operating cost since on $1 y$ the inexpensive filter medium is replaced.

The same high-voltage power supply can be used in both filter designs. Figure 91 shows that the filter design having the electrodes integrated with the filter medium will result in lower filtration costs than the design having electrodes separate from the media in those applications where filters are changed less frequently. The opposite is true in applications where filters are changed frequently. Since the frequency of filter changes is directly dependent upon particulate concentrations, the selection of the design type can be determined from an assessment of the particulate leveis. An important assumption in this design selection is that the filter medium is not cleaned after it is clogged.

High-Voltage Power Supply

Tne final component of the electric air filter is the high-voltage power supply. Our studies have shown that much higher filter efficiencies can be obtained using $D C$ rather than $A C$ voltage. The decreased filter efficiency with AC voltage is due to the elimination of the charged-fiber mechanism that results from the perlodic reversal of the electric field. The power supply must be capable of delivering the maximum DC electric field that can be applied across the filter medium without shorting. The electric field equals the applied voltage divided by the distance between the electrodes. For the same 


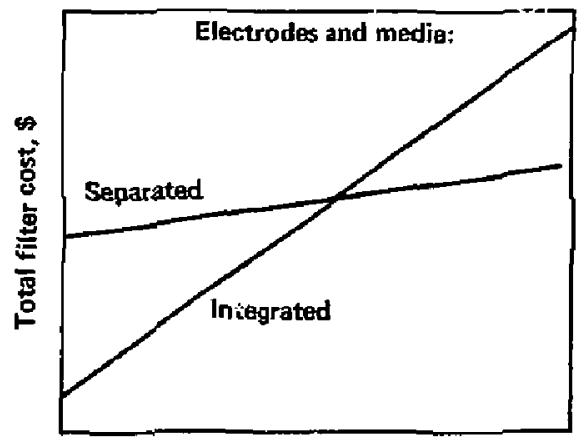

Fiitei changes/year

Fig. 91 Total filtration cost as a function of filter changes for electric filters having electrodes integrated with or separate from the filter media. 
electric field, the maximum voltage will be ?ow for electrodes ciosely spaced and high for electrodes spaced far apart. A cumpromise must be reached between the greater insulation required at higher voltages and the increased tolerance required for closely spaced electrodes. We selected $10 \mathrm{~mm}$ as the optimum electrode separation we could maintain at reasonable tolerances. At this separation, sparking occurs across the electrodes at 14-15 kV OC with the AF- 18 medium.

Although electric air filters require high voltage to generate a sufficiently high electric field, they do not require much current. In fact, most or the experimental tilters develaped in this program only require a few microamps of current. Because of the low current requirements, electric air filters can be made electrically safe without the use of complex interlocks or automatic shut-down circuits for overload conditions. The elimination of these componerits significantly reduces the complexity of the electric-filter power supply. Figure 92 (a) shows a typical pawer supply we used for our experimental filters. We designed the high-voltage power supply to coavert 110 volt $A C$ input to $10 \mathrm{kV} \mathrm{OC} \mathrm{output;} \mathrm{Fig} \mathrm{92(b)} \mathrm{shows} \mathrm{a} \mathrm{top} \mathrm{view} \mathrm{of} \mathrm{the} \mathrm{power} \mathrm{supply} \mathrm{with}$ the cover removed. The major component in this unit is a solid-state power supply obta:ined from Spellman. Since the power supply cannot exceed $70 \mathrm{uA}$ output currunt at $10 \mathrm{kV}$, the unit poses no hazard from electric shock. One can touch the high-voltage output and not feel the electrical discharge. He added high resistors to the output of the SPEl lman power supply to limit the current in case of an accidental overload. Although the miniature powe: supply is not capable of exceeding the $70 \mu \mathrm{A}$ output, the current limiters provide an additional safety factor. This power supply has separate push buttons to energize the unit and to supply high-yoltage output. The high-voltage switch also resets the high-valtage output if the power supply is overloaded; e.g., if the load on the power supply exceeds $70 \mu \mathrm{A}$. Indicator lights on the front paricl show if the high voltage is on or off.

The remaining des gn parameters in Table 7 describe perfomance and operating characteristics of the electric air filter. The characteristics werc fixed by the criteria we established for the physical dimensions of face velocity, filter medium, high-voltage electrodes, and the power sugply. These physical parameters determined the following parameters for the experimental filter: efficiency, dust-holding capacity, cost, and waste volume. The success of the eiectric air filter in meeting its objective to reduce the filtration cost and 

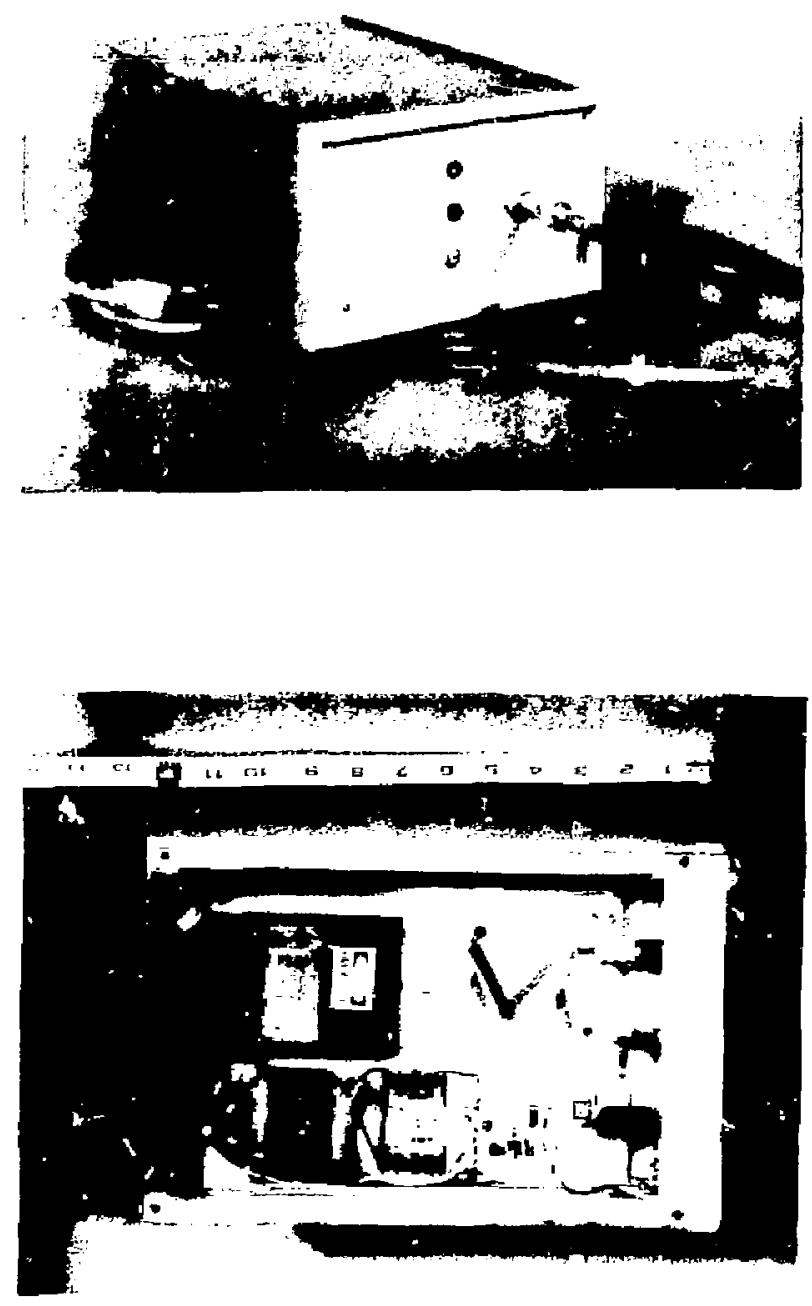

Fig. 92 High-voltage power supply for the electric air filter: (a) exterior $v$ iew and $(0)$ interior view with Spellman unit in lower right-hand corner. 
volume of radioactive waste depends uron these filter parameters. Although the addition of an electric air filter will generally extend the service life of a HEPA filter, it does not guarantee an overall reduction in the filter cost or the volume of radioactive waste collected. The added cost and waste collected due to the electric air filter may offset the savings made in the HEPA filter.

The design parameters we have discussed so far all dealt with the performance of the electric air filter. Although designing the filter to meet these performance criteria was a necessary condition, it was not sufficient to ensure widespread use in nuclear-ventilation systems. In addition to good performance, an electric air filter must be easy to operate and to be maintained by industry personnel (iable 7 ). Additionally, the high voltages empToyed in the use of the filter cannot create hazards for persannet.

\section{COST/BENEFIT ANALYSIS OF ELECTRIC AIR FILTERS}

Cost is an important design parameter in all of our electric air filter designs. Although we did not conduct a detailed cost/benefit analys is for each of the experimental filters described in this report, we recognized that the cost/benefit of a new filtration system must be significantly better than that of existing systems. Since the existing fijtration system in the nuclear ingustry consists of one or more HEPA filters, the cost/benefit of a new filtration system should be compared with HEPA filters. This comparison can be made in one or two ways depending upon the purpose of the new filtration system. If a new electric air filter replaces an existing HEPA filter, then a direct comparison can be made between the nes filter and the HEPA filter. If the new filter will act as a prefilter to the HEPA filter, then the comparison can be between the total cost of the prefilter-HEPA filtration system and the cost of a HEPA filter alone.

We developed a frmalized approach to determine the cost of a given fiitration system. Equation 62 determines the cost/benefit of replacing a HEPA filter with an electric air filter. On an annual basis, the totai filtration $\cos t, c_{T}$, for a given filter is

$C_{T}=N C+E / L$,

where $k$ is $t$.. number of filters used per year, $C$ is the operating cost of each 
filter, $E$ is the capital-equipment cost of the filter and support housing, and $L$ is the service life of the equipment.

For the case where an electric air filter is added as a prefilter to the HEPA filter, the total cost is

$C_{T}=N_{F} C_{F}+E_{F} / L_{F}+N_{H} C_{H}+E_{H} / L_{H}$,

where the subscript $F$ refers to the new "prefilter" and $H$ to the HEPA filter.

Equation 63 clearly points out that prefilters must sufficiently reduce the number of HEPA-filter changes to olarcone the increased cost due to the prefilters. The nunber of prefilters used per year may be determined from the

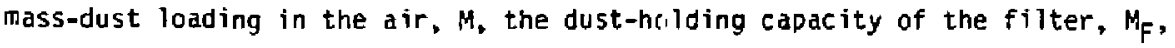
and the filter penetration, P:

$N_{F}=\frac{M\left(1-P_{F}\right)}{M_{F}}$

In a similar fashion the number of HEPA filters used per year is

$N_{H E}=\frac{M P_{F}\left(1-P_{H}\right)}{M_{H}}$

Since the penetration, $P_{H}$, for a HEPA filter is so small compared to 1 , Eq. 65 reduces to

$N_{H F}=\frac{M_{F}}{M_{H}}$

Substituting Eqs. 64 and 66 into Eq. 63 yields

$C_{T}=\frac{M\left(1-P_{F}\right)}{M_{F}} C_{F}+\frac{E_{F}}{L_{F}}+\frac{M P_{F}}{M_{H F}} C_{H}+\frac{E_{H}}{L_{H}}$.

Equation 68 includes capital-equipment cost, prefilter cleaning, recovery of material, and the effect of particle size. The operating cost of tri. prefilter then becomes

$C_{\Gamma}=C_{P F}+C_{L F}+\left(C_{C F}-C_{M} f M_{F}\right) n$

where $C_{P F}$ is the purchase cost of the filter; $C_{L F}$ is the labor cost to 
tesi. install, remove and dispose of the filter; $C_{C F}$ is the cost for cleaning the filter inplace or external to the ventilation system; $C_{M}$ is the cost per gram of material recovered from the filter; $f$ is the fraction of material removed from the filter; and $n$ is the number of cleaning cycles for the life of the filter.

The analogous equation for the operating cost for a HEPA filter is

$C_{H}=C_{P H}+C_{L H}$

For a cleanable filter, the dust-holding capacity is $M_{F} n$. Using these new values of dust-holding capacity and filter cost in Eq. 67 yields

$$
C_{T}=\frac{M\left(1-P_{F}\right)}{M_{F} n}\left[C_{P F}+C_{L F}+\left(C_{C F}-C_{M} f M_{F}\right) \pi\right]+\frac{E_{F}}{L_{F}}+\frac{M P_{F}}{M_{H F}}\left(C_{P H}+C_{E H}\right)+\frac{E_{H}}{L_{H}}
$$

Equation 70 represents the filtration cost in which we have allowed the filter to be cleaned and the trapped material to be recovered. It does not contain the important effect of particle size on the penetration, $P_{F}$, or the dust-holding capacity for the prefilter; $M_{F}$, or the HEPA filter, $M_{H^{*}}$.

The effect of particle size on the dust-holding -apacity, $M_{F}$ and $M_{H}$, is derived from our model of filter-clogging described earlier in this report. We have shown the equation for the pressure drop at low particle loadings (8q. 52).

The following relationship between particle volune fraction and particle mass, $M$, is

$$
M=a_{p} A z \rho_{p}
$$

where $A$ is the filter area, $z$ is the filter thickness, and $p$ is the density of the particles.

Substituting Eq. 7 into Eq. 52, we have

$$
\mathrm{M}_{\mathrm{F}}=\frac{\mathrm{r} \alpha_{\mathrm{F}} \mathrm{Ar} \cdot \rho_{\mathrm{P}}}{\mathrm{R}}\left(\frac{\Delta \mathrm{P}}{\Delta \mathrm{P}_{0}}-1\right)
$$


Equation 72 represents the pirticle-loading capacity for a filter. If the criteria for either replacing or cleaning the prefiter is $\Delta P=2 \Delta P_{0}$,

then $\mathrm{Eq} .71$ reduces to

$M_{F}=\frac{r \alpha_{F} A z \rho_{P}}{R}=K_{F} r$

where $K_{F}$ is a constant for a given filter.

Equation 73 shows that the particle-loading capacity, $M_{F}$, is directly proportional to the particle size; i.e., the smaller the farticle size, the lower the particle-holding capactiy.

This average size is a weighted air-resistance average 30 and is given by

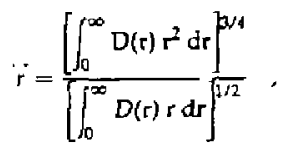

where $D(r)$ is the number-size distribution of particles trapped on the filter.

The distribution of particles trapped on the HEPA filter is given by the product ir $(r) P(r)$. Thus, $O(r)$ is equal to $N(r) P(r)$ for the HEPA filter. The distribution of particles trapped on the prefilter is $N(r)[1-P(r)]$ Thus, $D(r)$ is equal to $N(r)[(1-P(r)]$ for the prefilter. This term is grated over all particle sizes and aquals the mass of particles trapped on prefilter:

$\overline{\mathrm{M}} \overline{\left(1-\mathrm{P}_{\mathrm{F}}\right)}=\int_{0}^{\infty}\left(\frac{4}{3} \pi r^{3}\right) \rho_{\mathrm{P}} N(\mathrm{r})[1-\mathrm{P}(\mathrm{r})] \mathrm{dr}$

Equation 75 represents the difference in the size distribution of $p$ is before the filter, $N(r)$, and after the filter, $N(r) P(r)$. The mass particies trapped on the filter is obtained by multiplying this difference by no volume and density of each particle. A similar equation can be abtained foi ine mass of particles trapped on the HEPA filter: 
$\overline{M_{F}^{-}}=\int_{0}^{\infty}\left(\frac{4}{3} \pi r^{3}\right) \rho_{P} N(r) P(r) d r$

In general, the average particle size trapped on the HEPA filter is much smaller than the average particle size trapped on the prefilter. Because of the difference in average particle size between par cles trapped on the prefilter and the HEPA filter, different values of $r$ must aiso be used. This trenc caused some rosearchers to speculate that the use of a prefilter may accelerate the clogging of HEPA filters. This speculation, although not supported of experiments, is rased on ine fact that smaliar size particles result in a lower dust-holding capacity.

The effect of particle size on the cost equation for a prefilter-HEPA filter system is obtained by substituting Eqs. 73-76 into Eq. 70 to yield

$C_{T}=\frac{\overline{M\left(1-\bar{P}_{F}\right)}}{K_{F} \bar{T}_{F} n}\left[C_{P F}+C_{L F}+\left(C_{C F}-C_{M} K_{F} \bar{T}_{F} f\right)\right]+\frac{E_{F}}{L_{F}}+\frac{\overline{M_{F}}}{K_{H} \bar{r}_{H}}\left[C_{P H}+C_{L H}\right]+\frac{E_{H}}{L_{H}}$

The comparable cost equation for a single filter is

$C_{T}=\frac{\overline{M\left(1-P_{F}\right)}}{K_{F} \bar{r}_{F} n}\left[C_{P F}+C_{L F}+\left(C_{C F}-C_{M} K_{F} \bar{r}_{F} \hat{f}_{1} \bar{n}^{1}+\frac{E_{F}}{L_{F}}\right.\right.$

If the single filter is a HEPA filter, then $n=1, r_{C F}=0$, and $f=0$.

Filter cleaning has a major effect on the total cost of filtration. If the prefitite: $:$ not cleaned after it becomes clogged, then $n=1, C_{C F}=0$, $f=0$, and Eq. 77 reduces to cost Eq. 78 . In that case, the cost of replacing the prefilter pecomes the major variable affecting the overall cost. For example, if replacing the preiilter requires a major effort, such $\omega^{2}=$ bag-in and-out operation in a glove box or veitilation duct, then the labor costs, $C_{L F}$ will be high and the use of a prefilter will not be cost-effective. This condition has been a major factor preventing the use of pre-filters in the nuclear industry. However, if replacing the prefilter requires only a minor effort as a result of efficient prefilter and housing designs, then a prefilter 
can be very cost-effective, even with filter cleaning. This will be illustrated in the next section on applications of electric prefilters in glove boxes. In these applications, the prefilter is changed within one minute inside the glove box without interrupting the ventilation system or requiring a bag-in or sag-out operation.

Cleaning the prefilter can greatly reduce the cost if the cleaning costs, $c_{C F}$, are not high. Here again, the manner in which the prefilter is cleaned will control the overall cost-effectiveness uf the prefiltration system. If the prefilter has to be removed from the ventilation system and c:eaned in a remote location, then the cleaning costs will be very high. If the prefilter can be cleaned in sjtu or with only minor wark inside the enclosure containing the prefilter, then the cleaning costs will be low. We illustrate this practice in our glove box prefilters, where filter removal, cleaning, and reclacement is done entirely within the glove box.

cleaning the prefilter effectively increases the dust-holding capacit:y of prefilters by $n$ times and allows $i$. cased flexibility in the filter design. For example, a prefilter that cannot be cleaned must have a high dust-holding $\left.c a_{1}\right) a c i t y$. Unfortunately, most filter media with a high dust-holding caracity also have a high penetration, $P_{F}$, which leads to rapid clogging of the HEPA filter. However, by using a filter media with low penetration that $c$ an be cleaned, we can have both low penetration and high dust-holding capacity. Bag filters used in removing fly ash from coal-burning power plants are an excellent illustration of such a prefilter. Although the dust-holding capacity of a typical bag filter is low, the cumulative dust-holding capacity over thousands of cleaning cycles is axtremely high.

The recovery of radioactive material is another benefit of filter cleaning. The cost of recovered material like $P_{U}$ may significantly offset the cost of the fiitration system and even make the total filtration costs negative or permit profitabie operation. Even if the recovered material has no value, the value attributed to the material may be significant on the basis of material accountability, an important consideration in the nuclear industry.

\section{Service Life of Prefilter-HEPA Filter}

The extension of HEPA service life (HL) with the use of prefilters can be computed fron the ratio of the number of HEPA filters used without a prifilter, 
$\left(N_{H}\right)$, to the number of HEPA filters used with a prefilter, $\left(N_{H P}\right)$. Tris ratio is obtained from Eqs. 66 and 73:

$$
H L=\frac{N_{H}}{N_{H F}}=\frac{r_{A}}{r_{B}} \frac{1}{P_{F}}
$$

where $r_{A}$ is the average particle size after passing through a prefilter, $r_{B}$ is the average particle size before passing through a prefilter, and $P_{F}$ is the penetration of the prefilter. Equation 79 shows that the extension in HEPR iffe is primarily determined by the penetration of the prefilter. The ratio of the two particle sizes accounts for the linear variation in particle-loading capacity with particle size.

\section{Filter Waste}

Another major design parameter that nust be minimized is the volume of filter waste that is collected from beth electric air filters, or prefilters, and HEPA filters. . is is of special concern in the nuclear industry where discarded filters add to the inventory of radioactive waste. A formalized approach can be developed to determine the volume of waste generated by filters in a similar fashion to the approach used for determining filter cost. The volume equation for a single filter that is comparable to the cost Eq. 78 is

$V_{T}=\frac{\overline{M\left(1-P_{F}\right)}}{K_{F} \vec{I}_{F} n}\left(V_{F} g\right)$

where $v_{T}$ is tire total filter waste volume, $v_{F}$ is the original filter volume, and $g$ is the size reduction factor (other variables have beer defined). The equation for the total volume of filter waste for a prefilter-HEFw. filter systeris is

$V_{T}=\frac{\overline{M\left(1-P_{F}\right)}}{K_{F} \bar{T}_{F} \pi}\left(V_{F} g\right)+\frac{\overline{M P_{F}}}{K_{H} \bar{T}_{H}}\left(V_{H} h\right)$

where " $H$ is the volume of a HEPA filter and $h$ is size reduction factor for the yepA filter. 
From Eq. 81 , we see that the volume of total filter waste cen be reduced by increasing the loading capacity of both prefilter anu HEPA filter, $K_{F} r_{F}$ and $K_{H} r_{H}$, decreasing the penetration of the prefilter, $P_{F}$, increasing the number of cleaning cycles, $n$, and compacting the prefilter and HEPA filter for size reduction, $g$ and $h$. Cleaning, the prefilter can have a dramatic effect on the volume of filter waste, especially for high -efficiency prefilters. If the prefilter has a high number of cleaning cycles and a low penetration, then large reductions in filter volume can be achieved even if the prefilter has a low dust-holding capacity.

\section{Accidental Radiation Exposure}

Another major consideration in the cost/benefit study of air filcration systems in the nuclear industry is radiation exposure due to contaminated filter and accidental releases of radioactivity sen contaminated HEPA filters are replaced or cleaned. HEPA filters that are removed from glove boxes or ventilation ducts are carefully sealed in plastic bags to prevent radioactive dust from escaping into the atmosphere. However, despite numerous precautions, a significant number of radioactive spills in the nuclear industry occur during filter changes. The problem with these spills is not only the expensive cleanup of the contaminated area, but also the increased probability of raciation exposure to personnel by inhalation or ingestion.

Another source of radiation exposure and accidental releases of radioactivity occurs when clianging the HEPA filters in the large plenum chabers. In this case, workers enter the contaminated chamber wearing full protective suits with external air supplies. Changing HEPA filters under these conditions significantly increases the probability of radiation exposure during accidental spills. By decreasing the frequency of HEPA-filter changes thiough the use of prefilters, the risk of accidental spills can be reduced significantly.

A number of radionuclides like americium that are filtered out by HEPA filters have a high gamna emission. Because of the difficulty in shielding out this - liation, HEPA filters are generally replaced after a smuli quantity of gamm emitters is trapped on the filter. Arother reason for changing HEP? fil. before they reach their dust-holding capacity is liniting high-level radioaclive waste. If radioactive particles which have a high specific activity, 1 ike ${ }^{238} \mathrm{Pu}$, are loaded onto a HEPA filter, the HePA filter mLy be 
classified as high-level waste when discarded. Because of the significantly greater expense and ifficulty in isposing of high-level waste, HEPA filters are generally removec before accumulating that much radioactive materiat.

Since the maximum mass, $r_{R}$, of the radidactive material that yields acceptable radiation levels and waste criteria is much lower than the filter dust-holding capacity, $k_{H} r_{H^{*}}$ the HEPA filter has to be roplaced at a much higher frequency. The total cost and volume of waste may be computed by replacing $K_{H^{\prime}} r_{H}$ in Eqs. 77 and 81 with $M_{R}$. It is clear from the resulting equations that a prefilter would only be effective if it had an extremely low penetration value. Thus, for applications where radiation criteria are used for changing HEPA filters, a high-efficiency prefilter must be used which can be repeatedly cleaned or inexpensively removed. If the prefijter cannot be cleaned or easily removed, the advantage of a prefilter is lost since the radiation problem is transierred fron the HEPA filter to the prefilter.

Additional reductions in radiation exposure can also be expected because the probability of a spill occuring during the replacement of a prefilter is mucil less than during the replacement of a HEPA filter. Prefilters are generally much lighter and easier to handle than HEPA filters and are easier to bag-out of a ventilation system. Trefilters are also easier to replace than HEPA filters because the support and sealing structure for a prefilter is generally less complicated for a prefilter than for a MEPA filter.

\section{ELECTRIC AIR FILTERS FOR GLOVE BOXES}

\section{INTRODUCTION}

One of lire most attractive applications of an electric air filter in the nuclear industry is as a prefilter inside a glave box. We felt that the design of our experimental glove-box prefilters had to Jeet two requirements in order to be successful: (1) simple operation for replacing or cleaning filter media by untrained operators and (2) prefilters must be mounted inside the gln: $c$ oox to contain all of the radioactive material within the box. Proper design would allow the operator of the glove box to change or clean the fllter media in less than one minute without additional help. Also, this operation would not interrupt the wark going on inside the glove box. Moreover, by maintaining sma!l supply of clean fiiters inside the glove box, a number of fijter changes 
can be made withnut bag-in or bag-out operations.

To date, we have built and evaluated two experimental electric air filters for use as prefilters inside glove boxes. We designed both of these units so that each can be mounted on either a ceiling or a wall. The primary difference between the two designs is the mechanism that provides access to the filter riedium. The first design has a lever arm to release the ground electrode; the second uses a hinged door. The exposed electrode for safety reasons in both designs is the ground electrode. We also examined the safety features of the glove-box prefilters and incrporated the following features to reduce the possible high-voltage hazard: strong irsulation on all high-voltage lines, inter lock mechanisms on fitter-access doors, automatic power shut-off when the current exceeds a given level, and a flast', 19 yellow warning light. We liacer removed the interlock mechanism and flashing yellaw light when the electrical hazard was eliminated by using a current-limited mower supply.

\section{GLOVE-BOX PREFILTER USING A LEVER ARM}

\section{Description}

Figure 93 shows our first experimental electric air filter installed inside a glove box. This experimental unit consists of a polyethylene main body supported near the ceiling of the glove box, a high-valtage electrode mounted within the main body, a grounded electrode connected to a Tever arm on the main body, and a handle that opens and closes the filter assembly for changing filters. The electrodes, made from perforated aluminum $p l$ ates, have $a 40 \%$ open area. These electrodes were coated with 25 um-thick parylene to reduce the possibility of electric sparks. We had plared an insulation coating on these electrodes before we realized the negative effect it had on filter performance. Since we did not observe this detrimental e:fect in this prefilter, the electrodes apparently were not completely insulated. Independent tests on other parylene-coated electrodes indicate that the insulation coating had been partiatly removed, presumahly by mechanical abrasion.

We used two layers of $6.4 \mathrm{~mm}$ thick AF-18 obtained from Johns-Manuille for the filter medium (th) tab allows easy replacement). The prefilter was electrified with a Spellman miniature OC power supply. Model UM15-1500-0, that had a negative polarity and was rated up to $15 \mathrm{kV}$ at 75 .. 


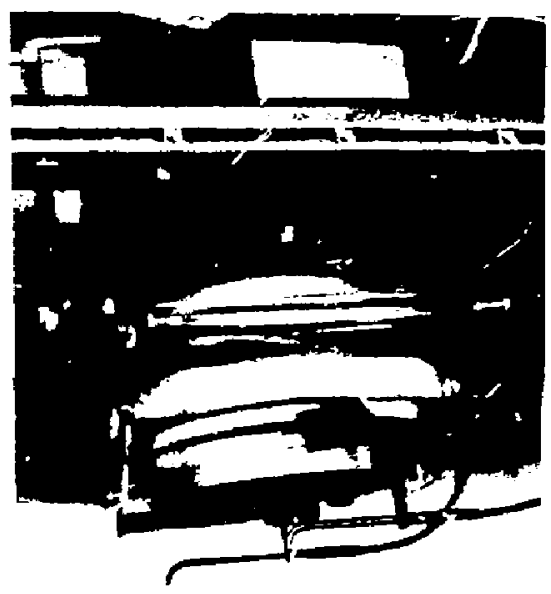

fig. 93 Experimental electric air filter of the lever-arm design installed in a glove box where it acts as a prefilter to the HEPA filter. 
Figure 94(a) shows an operator pulling the handle that releases the lower grounded electrode. Simplicity in changing the filter medium was our primary interest. A cam mechanism on the lever arm secures the ground electrode in its locked position. By pushing up on the handle, the ground electrode is lowered, thus exposing the filter medium. The operator can then remove the filter medium, as shown in Fig. 94(b).

It became apparent soon after we began our evalution that filter changes were more difficult than we had anticipated. The air flow through the filter medium tended to keep it pressed firmly against the upper electrode. To pull the filter medium free, we had to use tweezers to lift the medium. We al tried taping a short tab on the filter as shown in Fig. 94, but this tended to tear the medium after several pulls. We eventually used tweezers to pull the medium and this only added a few seconds to the changing operation. In other installations, "he use of a damper to reduce the air flaw during filter changes would eliminate this problem.

He also found that the cam mechanism did nct provide sufficient compression to seal the filter medium, and we had to insert a wedge between the handle and the ceiling of the glove box. This problem can be eliminated in other chesigns with a cam that provides greater compression.

Another problem with the lever-arm design was the ground electrode. We had used a perforated aluminum plate that had a $40 \%$ open area for our ground electiode. When this electrode was pressed firmly against the filter medium, $i t$ acted as a template for particle deposits. With oniy $40 \%$ of the filter surface available for particle veposits, the pressure drop would inciease much faster than if the entive surface had been available. However, the template effect due to the electrode was not important for most of our laboratory evaluations because we had used a thinner f:lter medium that did not make contact $\mathrm{w}^{\prime} \mathrm{i}$ "i the ground electrode. The additional separation made the entire filter surface available for particle deposits. Unfortunately, the loss of electrode contact may have decreased the performance of the electric air prefilter.

\section{Laboratory Evaluation}

Before we installed the experimental filter inside an active glove box, we evaluated its performance inside a controlled glove hox in which we generated 

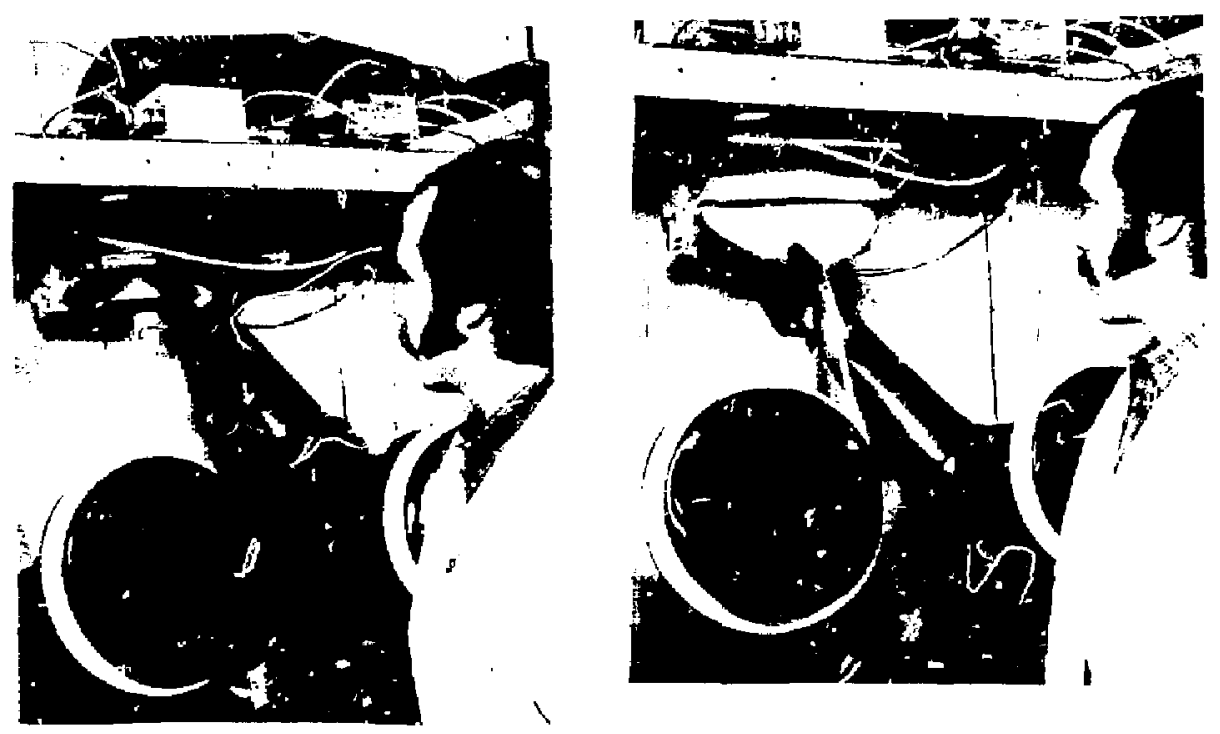

Fig. 94 (a) Operator pulling down on handle to change filter medium from the filter medium, ars (b) Operator remcving filter medium from the filter holder. 
$\mathrm{NaCl}$ aerosols by nebutizing a $1 \% \mathrm{NaCl}$ salution to yield a heterodisperse- $\mathrm{NaCl}$ distribution with a mass median aerodynamic diameter (MMAD) of $i .0 \mu \mathrm{m}$ and a geometric standard deviation, $\sigma_{g}$ of 2.0. Laboratory filter efficiencies were determined with a flame photometer obtained from Dynatech-Frontier, Inc., Albuquerque, New Mexico. This instrument photometrically measures the yellow Na 1 ight emitted when $\mathrm{NaCl}$ aerosols pass through a propane burner. (Filter efficiencies in the field evaluation are determined by using two in-line filter holders to sample the aerosols before and after the electric air filter simultaneously; this avoids the problem of large fluctuations in aerosol concentration.)

Since we wanted to make a larye number of efficiency measurements without the complications of bag-in and bag-out operations, we built a special sampling chamber to house the filter holders for sampling before and after the electric air filter. The sample filter holders are changed by access through two 1 atched doors.

To ensure that we obtained accurate filter-efficiency measurements with our sampling system shown we simultaneausly measured filters with the flame photometer and the in-line filters. These test measurements were made in our controlled glove box using $\mathrm{NaCl}$ aerosols. We determined he efficiencies with the in-line filters of atomic absorption analysis for Na. Figure 95 shows a series of efficiency measurements we made using the two techniques at applied voltages of $0,5,10$ and $15 \mathrm{kV}$. The average percent deviation between the two measurement techniques for all of the measurements, disregarding sign, was 7.6\%. Although this deviation is significant, we felt that the agreement of the intline filters with the flame photometer was close enough to be used for field evaluations.

\section{Field Evaluation in LLNL 's Uranium Powder Milling Box}

We installed the experimental electric air filter inside a glove box at LLNL where chunks of uranium and beryllium oxide ( $\mathrm{UO}_{2}$ and $\left.\mathrm{BeO}\right)$ are ground into a fine powder using a ball mill grinder. This glove box was one of several boxes used to manufacture reactor fuel rods. Since filtered room air was used to vent the box, the relative humidity inside the box was relatively low, thereby providing an ideal envi. ament for our evaluation. The electric air filter was sounted on a ceiling exhaust port; a plastic tube was mounted on 


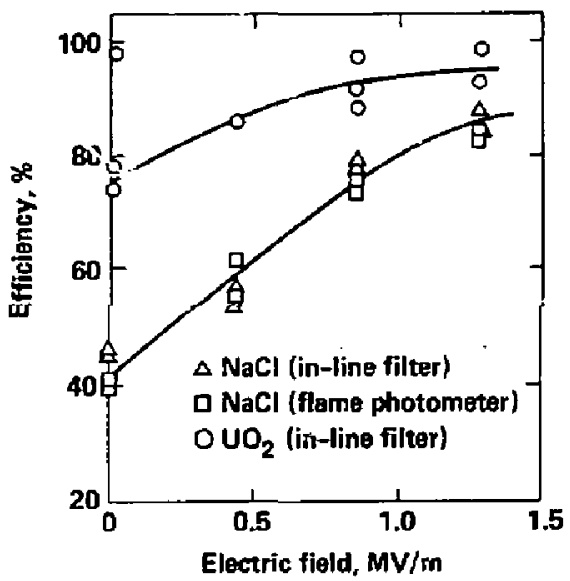

Fig. 95 Efficiency of pratatype filter using lever arm design as a function of applied electric field. 
the lower electrode to provide a representative sample of $U$ aerosuls before the filter (this tube prevented erronedus filter-efficiency measurements). A butterfiy valve located just outside the box allowed us to control the exhaustair flow from about $28.3 \mathrm{l} / \mathrm{s}$ to $4.7 \mathrm{l} / \mathrm{s}$. We maintained a flow of $23.6 \mathrm{l} / \mathrm{s}$ through the filter at all times by periodically adjusting the butterfly valve; this flow corresponded to the rated flow through typical HEPA filters used to filter glove-box exhaus:. An electric balance, accurate to $0.1 \mathrm{~g}$, was inside the glove box and allowed us to periodically weigh the filter.

We concucted our initial filter evaiuation in the milling box using two layers of AF-18 media. We selected this media because comparable tests with $\mathrm{NaCl}$ showed significant electrical enhancement and still allowed sufficient aerosols to pass through the filter for accurate efficiency measurements. At a filter face velocity of $65 \mathrm{~cm} / \mathrm{s}$, the filter efficiency for $\mathrm{NaCl}$ aerosols increased from $55 \%$ to $85 \%$ as we applied $10 \mathrm{kV}$ to the high-voltage electrodes.

The results of our first evaluation are summarized in Table 8 . Our evaluation consisted of periodically weighing the filter medium and measuring the filter pressure drop and efficiency. By taking filter samples of the aerosols before and after the electric air filter and by measuring the activity on a proportional counter, we determined filter efficiency for $\mathrm{UD}_{2}$ aerosols. Beryllium analysis on the same filter samples gave us identical results.

Perhaps the most surprising result in this first test was the unexpected high efficiency. The explanation for the high filter efficiency was the large size of the aerosols in the box. The AF-18 medium is considered to be poor filter medium and is used primarily in coarse filtration. We measured the size distribution of the radioactive aerosols with an Andersen impactor and found the activity median aerodynamic diameter (AMAD) was $5.4 \mu \mathrm{m}$, with $x=2.0$. Comparable measurements on $\mathrm{NaCl}$ aerosois showed a MMAD of $1.0 \mathrm{\mu m}$. Figure 96 shows the size distribution of the $\mathrm{UO}_{2}$ and $\mathrm{NaCi}$ aerosols with the complative percent of mass or activity plotted as a function of the aerodynamic diameier (the $\mathrm{NaCl}$ distribution was determined from mass measurements, while the $\mathrm{UO}_{2}$ distribution was determined from activity measurements).

We decided to use only one layer of AF-18, instead of two, in order to increase the accuracy of the filter-efficiency measurements in subsequent filter tests. Accurate efficiency measurements were not possible because of the unexpected high efficiency of even the mechanical filter for the uranium aerosols. Background radiation prevented accurate radiation counts on the 
Table 8. Performance of electric air filter in $U 0_{2} / 3 e 0$ glove box (two layers of AF $-18, V=0.65 \mathrm{~m} / \mathrm{s}$ ).

\begin{tabular}{cccc}
\hline $\begin{array}{c}\text { Particle Mass } \\
(\mathrm{g})\end{array}$ & \multicolumn{2}{c}{ \% Efficiencies } & \multicolumn{1}{c}{$\Delta \mathrm{P}$} \\
\cline { 2 - 3 } & $0 \mathrm{kV}$ & $10 \mathrm{kV}$ & \\
\hline 0.0 & 92.9 & 97.2 & 0.144 \\
4.3 & 97.5 &.--- & 0.197 \\
8.4 & 99.0 & 99.7 & $0.498^{\mathrm{a}}$ \\
$2.5^{\mathrm{b}}$ & $\cdots$ & $\cdots-.-$ & $0.197^{\mathrm{b}}$ \\
\hline
\end{tabular}

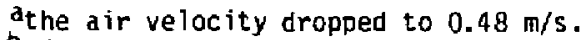

bafter shaking off 5.9 grams.

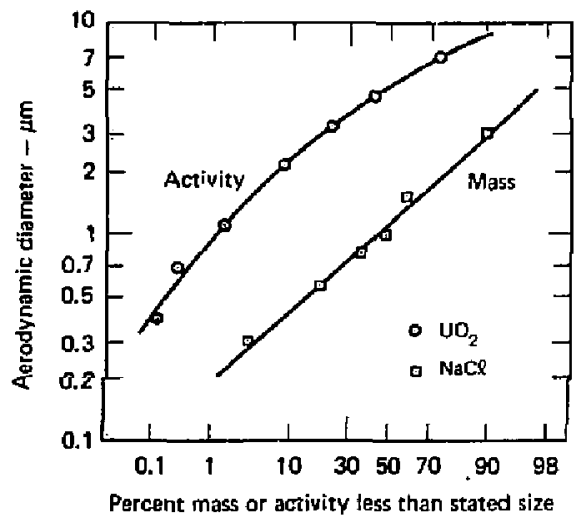

Fig. 96 Particle-size distribution for $1 \mathrm{NO}_{2}$ and $\mathrm{NaCl}$ aerosols for first evaluation of LLNL glove-box electric air filter. 
downstrean filter sample whenever the electric air filter had efficiencies in excess of $99.7 \%$.

At the time, we did not recognize the full consequence of using the thinner medium. The thinner medium did not have direct contact with both electrodes. This lead to a time-dependent deterioration of the electric-field enhancement; after several hours, the enhancement reduced ta zero. We did not see this change here because our measurements were relatively short. Because of the deterioration of the electric-field enhancement with time, the electric air filter would not show a significant increase in service life as compared to a conventional fibrous filter. We decreased the air velocity through the filter from $65 \mathrm{~cm} / \mathrm{s}$ to $52 \mathrm{~cm} / \mathrm{s}$ to allow additional flow control during inigher particle lcadings.

The second evaluation of the electric air filter under the new experimental conditiañs is summarized in Table 9. Table 9 shows three sets of efficiency and pressure-drop measurements in which 0 and $10 \mathrm{kV}$ was applied to the electrodes during filter-clogging. Each data set was generated with a singTe Af-18 filter in the manner previously described. The data set with no applied voltage represents the baseline test for comparison with the electric air filter. Note that we were unable to complete the second loading test with $10 \mathrm{kV}$ and had to terminate the test after accumulating only $1.1 \mathrm{~g}$ on the filter because the fuei-rod program was terminated during our evaluation.

Data Analysis. A review of the data in Table $g$ indicates considerable variation in the efficiency for the three data tests. The extent of this variation is readily seen by comparing the efficiencies of the tests having no particle mass. Ideally, all three data sets should have identical efficiencies at the different applied voltages. The most glaring discrepancy is the unusually high efficiency for Test 2 at $0 \mathrm{kV}$. We believe the most likely explanation for this high efficiency is a leak in the downstream filter holder. Al though we recognized the potential for leaks, we did not systematically check for leaks in this field evaluation. Except for this single high-efficiency value, the variation in the remaining data was probably caused by variations in particle size. We believe that the size of the aerosols produced in the glove box changes with the different operations performed.

We plotted some of the data from Table 9 in Figs. 95 and 97 to illustrate the two primary characteristics of electric air filter: the higher efficiency ifig. 95) and the fncreased service life (Fig. 97). As we have seen, Fig, 95 
Table 9. Performance of electric prefilter in $\mathrm{UO}_{2} / \mathrm{BeO}$ glove box (one layer of $\mathrm{AF}-\mathrm{lB}, \mathrm{V}=0.52 \mathrm{~m} / \mathrm{s}$ ).

\begin{tabular}{|c|c|c|c|c|c|}
\hline $\begin{array}{c}\text { Particle mass } \\
\text { (a) }\end{array}$ & $0 \mathrm{kV}$ & $5 \mathrm{kV}$ & $\begin{array}{c}\text { Efficienc } \\
10 \mathrm{kV}\end{array}$ & $15 \mathrm{kV}$ & $\stackrel{\Delta P}{(k P A)}$ \\
\hline Test 1 & \multicolumn{5}{|c|}{$0 \mathrm{kV}$ Applied During Particle Luading } \\
\hline 0.0 & 78.0 & --- & 97.9 & 99.1 & $0.052-0.060$ \\
\hline 2.3 & 98.4 & $99.9^{\mathrm{a}}$ & $>99.5^{b}$ & $>99.9^{b}$ & 0.070 \\
\hline $0.6^{c}$ & --- & --- & ---- & -.-- & $0.062^{\mathrm{C}}$ \\
\hline 5.6 & $99.8^{\mathrm{a}}$ & $99.8^{\mathrm{a}}$ & $99.7^{\mathrm{a}}$ & $99.8^{\mathrm{a}}$ & 0.393 \\
\hline $2.0^{\mathrm{C}}$ & --- & $-\ldots$ & --- & --- & $0.082^{c}$ \\
\hline Test 2 & \multicolumn{5}{|c|}{$10 \mathrm{kV}$ Applied During Particle Loading } \\
\hline 0.0 & 97.9 & 86.0 & 91.2 & 92.9 & 0.065 \\
\hline 2.1 & $=--$ & $-\cdots$ & $-\cdots$ & --- & 0.097 \\
\hline 3.2 & 98.5 & $\ldots$ & 39.5 & $99.9^{a}$ & 0.112 \\
\hline 6.8 & 99.2 & 99.5 & $99.7^{\mathrm{a}}$ & $\ldots$ & $0.378-0.406$ \\
\hline Test $3^{d}$ & \multicolumn{5}{|c|}{$10 \mathrm{kV}$ Applied During Particle Loading } \\
\hline 0.0 & 73.7 & --- & 99.6 & --- & \\
\hline \multicolumn{6}{|l|}{0.062} \\
\hline 1.1 & 80.2 & $\cdots$ & 99.6 & --- & \\
\hline 0.070 & & & & & \\
\hline
\end{tabular}

a. Radioactive counts on the downstream sample were lower than background,

b. Background radiation prevented accurate efficiency measurements above $99.7 \%$.

c. After shaking off the particle deposits.

d. Test prematurely terminated because all work inside the glove box had stopped. 


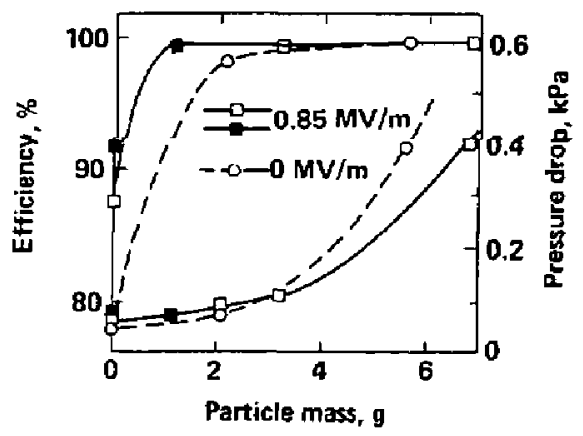

Fig. $97 \mathrm{Filter}$ efficiency and pressure drop for the experimental glove-box filter for three loading tests. 
shows a plot of the filter efficiency as a function of the applied electric field for clean filters. Since the distance between the electrodes was $0.00118 \mathrm{~m}$, we divided the applied voltage by this value to get the correct field strength. In addition to the $\mathrm{UO}_{2}$ data, we also included the $\mathrm{NaCl}$ data from our laboratory tests for comparison. Both data sets were generated with the same experimental fitter using a single 1 ayer of AF-18 medium.

Although the effect of the electric field appears to be significantly greater for the smaller $\mathrm{NaCl}$ aerosols than for the larger $\mathrm{UO}_{2}$ aerosols, this is not what occurs. The electric air filter reduces the penetration of both $\mathrm{NaCl}$ and $\mathrm{UO}_{2}$ aerosols by about $70 \%$. He should also point out that the $U$ data was obtained at $52 \mathrm{~cm} / \mathrm{s}$, while the $\mathrm{NaCl}$ data was obtained at $65 \mathrm{~cm} / \mathrm{s}$. However, other laboratory tests in which we studied the effects of air velocity between $65 \mathrm{~cm} / \mathrm{s}$ and $32 \mathrm{~cm} / \mathrm{s}$ nave shown the small decrase in air velocity for the $U$ aerosols is responsible for a slightly higher efficiency for the $\mathrm{UO}_{2}$ aerosals when high voltage is applied. The major contribution to the higher filter efficiency with $\mathrm{UO}_{2}$ aerosols is the ir larger size as compared to the $\mathrm{NaCl}$ aerosols. This fact is readily seen when comparing the filter efficiencies in Fig. 95 at $0 \mathrm{kV}$.

In addition to the increased efficiency, increased service life results when an electric field is applied across a fibrous filter. Figure 97 shows that the filter efficiency increases with particle mass and with an applied electric field. Although the pressure drop at higher particie mass loadings is slightly lower for the filter with an electric field than without, we expected the difference to be much greater. We believe the small value resulted from the lack of electrical contact of the filter medium with both electrodes. We have previously mentioned how this could lead to a gradual dissipation of the electric effects. Another possible calise for the lack of a more dramatic difference in filter service life is the inconsistent use of high voltage in both filters to generate the data in Table 9 . Since none of the tests in Table 9 were conducted exclusively with or exclusively without high voltage, the service life of the filters in these tests fell between the two extremes, thus reducing the differences in service life due to the electrification. Figure 97 also shows that after $3 \mathrm{~g}$ of particles were collected by the filter, the efficiency for both thic standard and e'ectric air ijiter were nearly 100\%. The evaluation also showed that $70 \%$ of the radiaactive dust could be removed and the filter ned iuni placed back into service. 
Although our field evaluation has to stop after only three months because work had been terminated in the box, sufficient data was obtained to perform a cost/benefit analysis. The filter perfomed very well except for the use of a wedge to improve the compression seal and the small open area of the frant electrode as previousiy noted. The filter media was removed from the holder and replaced many times with little difficulty and presented no problem with the high-voltage system. We have already noted that future designs should provide a filter seal with greater compression and a means for reducing the pull of the media against the rear electrode for easier removal. Possible solutions for decreasing the pull are either reducing the exhaust flow by a throttle valve or providing a bypass for the exhaust air during media replacement. The electrical system had also functioned well during the evaluation without the occurrence of a single spark or overlaod. Had either of these events occurred, the high voltage power supply would have shut down.

We performed our cost/benefit analysis of the experimental filter using the field evaiuation data in Fig. 97. Unfortunately, we were unable to measure the HEPA-l ife extension directly because we did not have an accessible HEPA filter downstream of the electric prefiltar. To compensate for this lack of baseline data, we used data reported by Adley and wisehart ${ }^{6.3}$ that correspanded closely to the experimental conditions of our field evaluation.

Adley and Wisehart had found that the standard 1000-cfm HEPA filter had a dust-holding capacity of $2000 \mathrm{~g}$ for $5.5 \mu \mathrm{m}$-diameter particles and $300 \mathrm{~g}$ for $0.5 \mu$ rn-diameter particles. The mass median diameter of the $\mathrm{UO}_{2} / 8 \mathrm{eO}$ particies in our evaluation was $5.4 \mu \mathrm{m}$. Although we did not measure the particle-size distribution after the air filter, a mass median diameter of $0.5 \mu \mathrm{m}$ is very roasonable. By scaling down the capacity of the 1000-cfm HEPA to air-gquivalent $50 \mathrm{cfm}$ HEPA, the dust-holding capacity is $100 \mathrm{~g}$ for $5.5 \mathrm{\mu m}$ particles and $15 \mathrm{~g}$ for $0.5 \mathrm{~mm}$ particles.

We determined the remaining parameters needed for the cost/benefit analysis from the performance date in Fig. 97 where the mechanical prefilter had an average lifetime efficiency of $96 \%$ and a dust-holding capacity of $5.6 \mathrm{~g}$. The corresponding average efficiency and dust holding capacity for the electric air filter is $99 \%$ and $6.8 \mathrm{~g}$ respectively. The prefilter cost approximately $2 d$ and had a volume of 0.211 . During the field evaluations, we 
also found that $67 \%$ of the particles could be removed from the prefilter by mild mechanical shaking and reusing the filter. The last variable used in our cost/benefit analysis was the factor describing the reduction in volume by compressing the filter. This factor was 0.16 for the prefilte: medium and 0.45 for the HEPA filter. We have listed all of the variables used in our cost/benefit study in Table 10.

We analyzed the filtration cost for the prefilter-HEPA filter system and for the HEPA filter alone using cost/benefit equations previously discussed. In both cases, we neglected the labor cost and also neglested the cost of clealing the prefilter and the value of the material recovered. Since the labor cost for replacing the HEPA filter greatly exceeds the cost for prefilter cleaning and media replacement, the onission of these costs does not affect the final results that show that using a prefilter is very cost effective. Including the labor costs in the total cost analysis would, in fact, make the use of prefilters even more cost effective. We also neglected the cost for the prefilter holder and power supply and the HEPA filter holder. These capital equipment costs have not been estimated since there are no commercially available electric air filters to establish a cost basis.

The total cost for the HEPA filter is $\$ 27.00$ since we arbitrarily selected a total annual dust load of $100 \mathrm{~g}$ of $5.5 \mu \mathrm{m}$ diameter particles which corresponds to the life of one HEPA. The cost for the prefilter-HEPA filter was calculated for both the mechanical prefilter and the electric prefilter for the case with no prefilter cleaning $(n=0)$ and with ten prefilter cleanings $(n=10)$ before the prefilter was discarded. We made a similar analysis of the waste volume.

The results of these cost and volume calculations are tabulated in Table 11; Table 11 also shows the amount of material recovered and the extension in HEPA 1 ife.

The cost of the filtration system jecreases very rapidly from $\$ 27.00$ to $\$ 7.54$ and finally to $\$ 2.09$ when using a mechanical prefilter and electric prefilter, respectively. Note that cleaning the prefilter has very little effect on the cost of the system due to the insignificant cost of the prefiiter media. in contrast to the cast, the volume of fiiter waste decreases more slowily from 6.29 to 5.281 and 3.481 , as the mechanical prefilter and electric prefilter are added, respectively. Either cleaning or compressing the prefilter results in a significant reduction in the volume of waste. However, 
Table 10. Variabies used in the cost/benefit analysis of electric prefilters in $\mathrm{VO}_{2}$ glove box.

\begin{tabular}{|c|c|c|c|c|}
\hline \multirow[b]{2}{*}{ Variable } & \multicolumn{2}{|c|}{ Prefiltera } & \multirow{2}{*}{$\begin{array}{c}\text { HEPA } \\
\text { (after pref } i \text { ?ter) }\end{array}$} & \multirow{2}{*}{$\begin{array}{l}\text { HEPAt } \\
\text { (alone) }\end{array}$} \\
\hline & Mechanical & Electrical & & \\
\hline$c_{\mathrm{PF}}, \mathrm{c}_{\mathrm{PH}}$ & $\$ 0.02$ & $\$ 0.02$ & $\$ 27.00$ & $\$ \div \% .00$ \\
\hline$V_{F}=V_{H}$ & 0.211 & 3.271 & 6.291 & 6.291 \\
\hline$P_{F}, P_{H}$ & $\begin{array}{l}0.04 \\
100 \mathrm{~g}\end{array}$ & $\begin{array}{l}0.01 \\
100 \mathrm{~g}\end{array}$ & $\begin{array}{l}0.00 \\
100 \mathrm{~g}\end{array}$ & $\begin{array}{l}0.00 \\
100 \mathrm{~g}\end{array}$ \\
\hline$M P_{F}$ & $\cdots$ & -- & $4 \mathrm{~g}, 1 \mathrm{~g}^{\mathrm{c}}$ & $100 \mathrm{~g}$ \\
\hline$M\left(1-P_{F}\right)=M\left(1-P_{H}\right)$ & $96 \mathrm{~g}$ & $99 \mathrm{~g}$ & -- & $\cdots$ \\
\hline$r_{F}, r_{H}$ & $2.25 \mu \mathrm{m}$ & $2.25 \mu \mathrm{m}$ & $0.25 \mu \mathrm{m}$ & $2.25 \mu \mathrm{m}$ \\
\hline$k_{F} r_{F}, k_{H}, r_{H}$ & $5.6 \mathrm{~g}$ & $6.8 \mathrm{~g}$ & $15 \mathrm{~g}$ & $100 \mathrm{~g}$ \\
\hline$f$ & 0.67 & 0.67 & 0 & 0 \\
\hline 9 & 0.16 & 0.16 & $C .45^{d}$ & $0.45^{0}$ \\
\hline
\end{tabular}

a variable subscript is $F$.

b variable subscript is $H$.

c dust load on the HEPA is $4 \mathrm{~g}$ using the mechanical prefilter and $1 \mathrm{~g}$ with the electrical prefilter.

if the wooder. frames are burned, then the volume reduction fracting is 0.11. 
Table 11. Calculated annual performance of glove-box filtration systems based on experiments in $\mathrm{UO}_{2}$ powder milling box.

\begin{tabular}{|c|c|c|c|c|}
\hline \multirow[b]{2}{*}{$\begin{array}{c}\text { Filtration } \\
\text { System }\end{array}$} & \multicolumn{4}{|c|}{ Performance Criteria } \\
\hline & Cost & $\begin{array}{l}\text { Voiume of waste } \\
\text { (with compression) }\end{array}$ & $\begin{array}{l}\text { Material } \\
\text { recovered } \\
(100 \mathrm{~g} \text { input })\end{array}$ & $\begin{array}{l}\text { HEPA } \\
\text { Life }\end{array}$ \\
\hline HEPA & $\$ 27.00$ & $\begin{array}{ll}6.29 & 1 \\
2.83 & 1)\end{array}$ & و & $1 x$ \\
\hline $\begin{array}{l}\text { MEPA plus } \\
\text { Mecharical Pre- } \\
\text { Filter }(n=1)\end{array}$ & $\$ 7.54$ & $\begin{array}{ll}5.28 & 1 \\
(1.34 & 1)\end{array}$ & 09 & $2.8 x$ \\
\hline $\begin{array}{l}\text { HEPA p Tus } \\
\text { Mechanical } \\
\text { Prefiltar }(n=10)\end{array}$ & $\$ 7.23$ & $\begin{array}{ll}2.04 & 1 \\
(0.82 & 1)\end{array}$ & $6 ? \mathrm{a}$ & $2.8 \mathrm{x}$ \\
\hline $\begin{array}{l}\text { HEPA plus } \\
\text { Electric } \\
\text { Prefilter }(n=1)\end{array}$ & $\$ 2.09$ & $\begin{array}{c}3.48 \quad 1 \\
(0.501)\end{array}$ & $0 \mathrm{~g}$ & $11.1 x$ \\
\hline $\begin{array}{l}\text { HEPA plus } \\
\text { Elact "ic } \\
\text { Prefilter }(n=10)\end{array}$ & $\$ 1.83$ & $\left.\begin{array}{ll}0.73 & 1 \\
(0.24 & 1\end{array}\right)$ & $69 \mathrm{~g}$ & $11.1 x$ \\
\hline
\end{tabular}


compressing the filter after cleaning reduces the waste volume by only a small amount. Table 11 also shows that the only recovery of material occurs when the prefilter is cleaned. The amount of material recovered is about the same for both the mechanical and the electric prefiltration. In these calculations, we assumed the HEPA filter cannot be cleaned. The last performance criterion listed in Table 11 is the HEPA filter life. Note that HEPA life is inversely proportional to the cost of the filtration system and increases to 2.8 and 11.) times the original HEPA 1 ife as a mechanical prefilter and an electric prefilter are added.

It is important to recognize that the large difference in filter cost and HEPA 1 ife between the filtration systems using a mechanical prefilter and the electric air filter is primarily due to the difference in efficiencies shown in Fig. 97. Note that the transient period where the efficiency rapidly increases as particle mass accumulates is the dominant factor controling the average efficiency, and the HEPA 7 ife. We have already noted that the lack of electrode contact and the mixing of high voltage and zero voltage conditions reduced the difference in performance between the mechanical and electrical prefiiter. We would expect much greater extension in HEPA service life for a prefilter that was electrified all of the time. In reviewing the results of the $\mathrm{UO}_{2}$ glove-box evaluation, we must also remember that the MMAD was $5.4 \mu \mathrm{m}$. This size aerosol is easily removed by a large number of methods. The significant savings in cost and extension of the HEPA service life for the mechanical prefilter is primari?y the result of this large particle size. If ihe $U$ aerosols were significantly smaller, then the mechanical prefilter would have a much smaller savings and only a modest extension in HEPA life. The performance of the electric air filter acting as a prefilter would have its most dramatic effect below $1.0 \mu \mathrm{m}$ when all other methods become less efficient.

GLOVE-BOX PREFILTER WITH A HINGED DOOR

\section{Description}

The second experimental filter we designed is shown in Fig. 98. Although Fig. 98 shows the unit attached to the end-plate of the glove box, it could also be mounted on the ceiling as in the other filter design. The hinged-door 


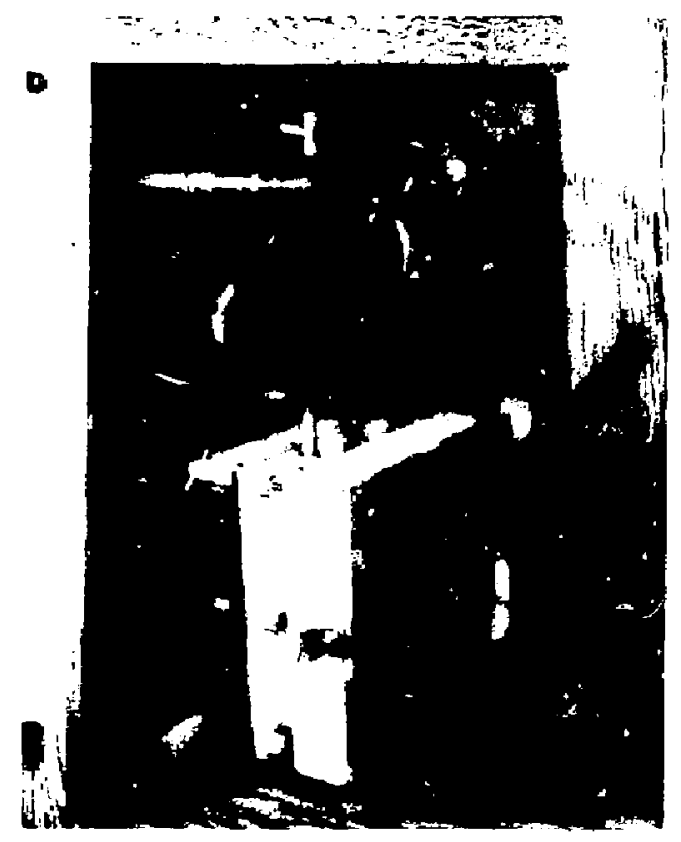

Fig. 98 Electric air filter with hinged door mounted on end plate of glove tox. 
unit is much larger and more complicated than a production unit because it has built-in probes to sample aerosals before and after the filter. We added a plastic shroud around the inlet of the filter to obtain isokinetic samples. We had observed in our last evaluation, that, without a shroud, we would get erroneousily low inlet aerosol concentrations due to bypass around the sample probe. If aerosol samples ere not required, the thickness of the electric filter could be reduced to less than $50 \mathrm{~mm}$.

The important design features of this hinged-door electric prefilter are the two latches that provide easy access to the filter medium and HEPA filter. The main body of the experimental unit is polyethylene which provides good insulation and strength. A metal reinforcement was required on the front plate to prevent distortion when the Tatch was closed. The filter medium used in this evatuation was the same as in our previous evaluation.

In designing this experimentai unit, we avoided the problems encountered with the previous design. Because of our previous experience with the upstream electrode masking off much of the filter surface, we used a comercially available wire screen with $80 \%$ open area. The downstream $h$ igh voltage electrode was a hand-strung grid with wires spaced every $10 \mathrm{~mm}$. We had initially used insulated wires for the rear high-voltage electrade in order to minimize the possibility of sparking. Sirce we i:scovered the negative effects of the insulated electrodes, we replaced the insulated electrode screen with non-insulated wires. The problem of inadequate filter compression encountered with our previous glove-box filter did not occur with this design because of $t^{t}:=$ increased compression of the hinged door.

The power supply used with this experimental filter was a modified Hipotronics AC-DC HIPOT tester, Model HO115, with a negative output polarity. We used this power supply described in the section on fundamental studies and found it to be ideally suited for wse in our experimental filters. The unit has a variable 0-15 kV UC output, a current meter and automatic shut-down during an overload condition when the current exreeded a preselected level.

To ensure safe operation during the field evaluation, we added a number of safety features were added to the electric filter and power supply. Two redundant magnetic reed switches were imbedded in each of the two doors for shutting down the high voltage whenever the filter door was opened. Heavy-duty cable was used for all of the exposed high-voltage lines to ensure that the insulation would not fail during the long field evaluation. A second current 
overload circuit was added to the power supply to ensure that the power would be shut off when the load exceeded 40 uA. As an additional safety precaution, a $10 \mathrm{M} \Omega$ currert- 1 imiting resistor was placed in saries between the power supply and the electric filter. This 1 imited the available energy in a potential spark whi'e providing sufficient current to trip the overload circuit.

\section{Laboratory Evaluation}

We tested the filters with heterodisperse $\mathrm{NaCl}$ aerosols and filter efficiencies were determined by sampling before and after the filter. We compared them using the flame photometer as a detector and the in-line fitters housed in the sampling chamber. We then conducted filter-efficiency measurements over a range of experimental conditians intended to encompass the actual conditions of the field evaluation. The test for one layer of AF-18 medium at flow velocities of $0.65 \mathrm{~m} / \mathrm{s}, 0.32 \mathrm{~m} / \mathrm{s}$, and $0.16 \mathrm{~ms}$ showed a large increise in filter efficiency that resulted then the flow velocity was decreased (Fig. 99).

\section{Field Evaluation in fu-Dust Vacuum Box at Racky Flats}

After the laboratory tests at LLNL, the electric air filter and end plate were shipped to the Rocky Flats Plant in Boulder, Colorado and installed in a dust-vacuum glove box. This glove box encloses the filter portion of a vacuum-cleaning system used to remove dust from a group of other glove boxes that extract Pu in molten salt operations. The vacuum-cleaning system consists of suction hoses inside the other glove boxes, connecting plumbing that leads to four cartridge filters and a vacuum turbine. Dust vacuumed from the other glove boxes is split into two parallel paths, each of which have two cartridge filters in series. The only time dust is generated inside the filter glove box occurs when the cartridge filters are cleaned or replaced. The atmosphere of the glove box is essentially pure nitroger, very dry, and is kept at room temperaisure.

Figure 100 shows a side view of the glove box with the vacuum turbine extending from the end plate to the right. The glove box is approximately a 3-ft cube. One of the four cartridge filters can be seen through the side 


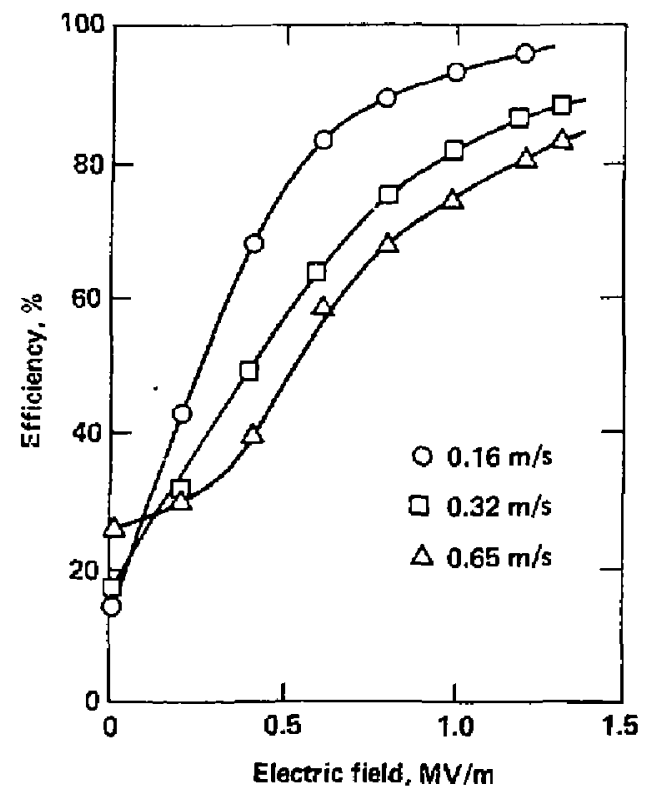

Fig. 99 Filter efficiency as a function of electric field for one layer of $A F-i 8$ medium tested at different flow velecities.

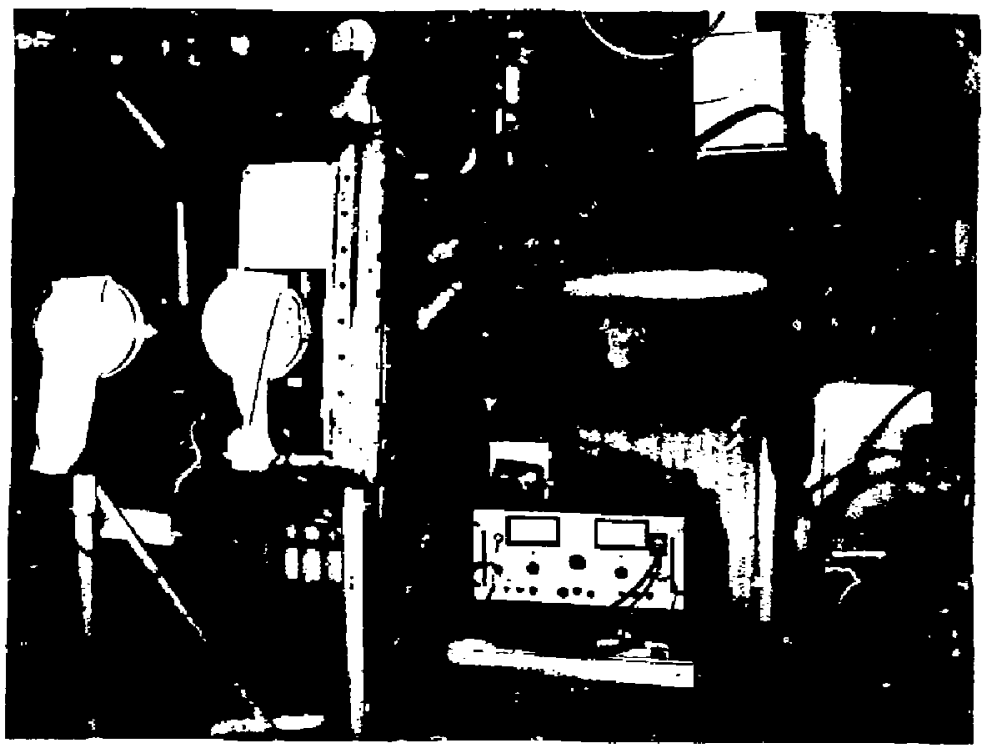

Fig. 100 Plutonium dust vacuum box housing the electric prefilter. 
window. Figure 100 also shows a portion of the electric air filter between the two glove ports, the sampling chamber mounted on top of the glove box and the high-voltage power supply. Exhaust from the glove box passes through the electric air filter and HEPA filter, and exits through the exhaust pipe. A butterfiy valve controls the exhaust flow, which can vary between 0 and $6.611 / \mathrm{s}$. The exhaust flow, which is measured with a Hastings velocity meter, is controlled by the exhaust vacuum source and th 2 total resistance due to the electric air filter, HEPA filter, butterfly vaive, and inlet restrictions to the box.

Since the maximum exhaust vacuum is normally $249 \mathrm{~Pa}$, the exhaust flow decreases as the filter resistance increases due to filter-clogging. When the toial resistance equals the 249-Pa-source vacuum, then the exhaust flow rate draps to zero. However, as the flow decreases to nearly zer , the glove-box vacuum remains nearly constazt. He discovered during our evcluation that when the filter was plugged and the exhaust flow was reduced to neirly rero, the glove box was still able to maintain a one-inch negative F-essure. This surprising behavior was due to complex external plumbing that restricts the inlet flow to maintain a constant glove-box vacuam. We should point out that such a system can lead to a potentially serious problem. For those glove boxes that have a variable inlet restriction, a negative vacuum is insufficient to enșure adequate protection during leaks and accidental breaks. In Jdition to the vacuum criterion, an exhaust flow criterion must be established to ensure adequate capacity.

We intended the evaluation of our electric air filter in the dust vacuum box to be more comprehensive than our previous evaluation in the uranium-powder milling box. The most important change in this evaluation was the addition of a standard $8^{\prime \prime} \times 8^{\prime \prime} \times 6^{n}$ HEPA filter as an integral part of the filtration system. This change enabled us to make direct measurements of the extension in the HEPA service life due to the electric air filter.

We evaluated the system by measuring the particle concentration before and arter the electric air filter and then measuring the weight of particle deposits on the electric air fiiter and HEPA filtor. We placed a balance inside the glove box for these weighinrs. The pressure drop across the HEPA and air filter was measured with a magnehelic and the exhaust flow was measured with a Hastings velocity meter before and after each filter weighing. 
We conducted the efficiency measurements by generating radiodctive dust inside the glove box through cleaning of the cartridge filters. These cartridges were readily cleaned by striking them firmly on the floor of the glove box to knock off particle deposits. After installing the clean cartridges and sweeping the dust into a canister, there was a sufficient amount of airborne dust to begin the efficiency measurenents. We initially made efficiency measurements at 0,5 , and $10 \mathrm{kV}$, but later changed to only one measurement at $10 \mathrm{kV}$ to simplify the procedure. Table 12 shows the results of these efficiency measurements for our electric air filter.

As in our previous evaluations in the $\mathrm{UO}_{2}$ glove box, we also weighed the filter to obtain the particle mass and measured the pressure drop. Note that we had to calculate the efficiency at $10 \mathrm{kV}$ for zero particle mass because we had inadvertentiy left the filter medium out of the sample holder. Table 12 also shows the efficiency of the electric prefilter after accumulating $5.6 \mathrm{~g}$ of particles. Because Pu has a high specific activity, we were able to accurately measure very high filter efficiencies.

Buring these measurements, we had not adjusted the butterfly valve to maintain a constant exhaust flow since the box had a constant negative pressure of one inch water. We did not anticipate the large decrease in flow that occurs when the filter becomes loaded with particle deposits. We assumed that the standard practice of maintaining a negative pressure inside the box would assume an adequate exhaust flow. Although the Hastings velocity meter had indicated a decreased flow, we disregarded the measurement because the readings were in serious error. The flow velocity at 0 g particle loading was determined to be $8 \mathrm{~cm} / \mathrm{s}$ based an the measured HEPA filter pressure drop of $12 \mathrm{~Pa}$ and the known relationship between flow velacity and filter pressure drop. The flow velocity at $5.6 \mathrm{~g}$ particle loading was estimated at $1 \mathrm{~cm} / \mathrm{s}$.

Results. A comparison of the filter efficiencies for the Pu salt aerosols in Table 12 with our previous tests on $\mathrm{NaCl}$ aerosols shows that the $\mathrm{Pu}$ aerosols have significantly higher efficiencies, especially at applied low voltages. The higher efficiencies for the Pu salt aerosols are due to their larg'r size. We measured the particle-size distribution of the Pu salt aerosols using the same Andersen impactor we used previously in the $\mathrm{UO}_{2}$ glove box. Thes: measurements showed the AMAO and geometric standard deviation were $3.5 \mu \mathrm{m}$ and 2.0, respectively. In comparisan, the $\mathrm{NaCl}$ aerosol had a iMAO 1.0 and a geometric standard deviation of 2.0. The higher efficiency of the larger Pu 
Table 12. Hinged-0oor electric air filter in a $P u$ glove box (two layers of AF-18, $V=$ variable ${ }^{a}, 10 \mathrm{kV}$ during particle loading).

\begin{tabular}{|c|c|c|c|c|}
\hline $\begin{array}{c}\text { Particle Mass } \\
\text { (g) }\end{array}$ & \multicolumn{3}{|c|}{ \% Efficiencies } & $\begin{array}{l}\Delta P \\
(k P A)\end{array}$ \\
\hline $\begin{array}{r}3 \\
5.6\end{array}$ & 92.6 & $\begin{array}{l}97.5 \\
---.\end{array}$ & $\begin{array}{l}99.2^{b} \\
99.97\end{array}$ & $\begin{array}{l}0.037 \\
0.199\end{array}$ \\
\hline
\end{tabular}

a $V=8 \mathrm{~cm} / \mathrm{s}(5 \mathrm{cfm})$ at $0 \mathrm{~g}$ loading, $V \sim 1 \mathrm{~cm} / \mathrm{s}(0.5 \mathrm{cfm})$ at $5.5 \mathrm{~g}$ loading b Calculated based on 0 and $5 \mathrm{kV}$ data. 
salt aerosols can be explained by the filter efficiency as a function of particle size. After first converting the Anderson impactor measurements to actual diameter measurements with a laser particle counter, we see that the $\mathrm{NaCl}$ aerosols have a mass median diameter of $0.68 \mu \mathrm{m}$ and an efficiency of 40\%. The corresponding mass median diameter for the Pu salts is $1.43 \mathrm{~mm}$, assuming a density of 6 , and an efficiency of $80 \%$. Although the measured efficiency at $0 \mathrm{kV}$ was higher $(92.6 \%$ instead of $80 \%)$, it is clear that the larger particle size of the $P_{t}$ salt aerosols accounts for the higher efficirncy as compared to $\mathrm{NaCl}$ aerosols.

In addition to the higher efficiency at $0 \mathrm{kV}$ as compared to the $\mathrm{NaCl}$ aerosols, the Pu salt aerosols also show a lower enhancement of the efficiency than what is predicted due to the applied voltage. Ccher tests show that the filter efficiency at $8 \mathrm{~cm} / \mathrm{s}(2.4 \mathrm{l} / \mathrm{s})$ only increases from $92.6 \%$ to $97.5 \%$ as $5 \mathrm{kV}$ is applied. In contrast, the filter efficiency fồ $\mathrm{NaCl}$ aerosols at $16 \mathrm{~cm} / \mathrm{s}$ increases from $34 x$ to $93 \%$ as $5 \mathrm{kV}$ is applied. The percent reducticn in filter penetration is $67 \%$ for the Pu salt aerosols and $89 \%$ for the idaci aerosols. However, based on our theoretical analys is of the effect of particle size and the applied electric field, the larger Pu salt aerosols should have a greater reduction in penetration than the smaller $\mathrm{NaCl}$ aerosols. The decreased flow velocity used in filtering out $\mathrm{Pu}$ salt aerosols should result in even higher efficiencies.

A possible explanation for the lower increase in filter efficiency than expected from laboratory experiments and theory is a high particle charge that has already converted the mechanical filter to an electrical filter. We have shown that the accumulation of charged particles on the fibers will significantly increase the filter efficiency. If the particles have a high enough charge, they will be attracted to the fiber even without an applied electric field. This hypothesis is supported by the higher efficiency at $0 \mathrm{kV}$ (92.6\%) than predicted from our laboratory studies (80\%).

Our first evaluation was terminated after obtaining the results shown in Table 12 because the pressure drop across the prefilter had secreased from 0.20 to $0.05 \mathrm{kPa}$ after weighing the filter. Although the cake of particle deposits on the filter surface did not appear to be disturbed during the weighing, we believed we had ruined the test and began a new evaluation with a fresh filter. We later found that such drastic changes in the pressure drop occur quite frequently. Small vibrations would create small cracks in the particle cake 
and restilt in large decreases in the filter pressure drop.

We had intended to obtain sufficient data for generating curves of filcer efficiency and pressure drop as a function of particle mass loading for the electric and mechanical filters as we had done in the LLNL glove box. A seriolis problem we encountered was the lack of accurate flow measurements. Since the efficiency and pressure drop across any filter is a function of the face velocity of the gas being filtered, it is important to measure the exhaust flow rate. Unfortunately, during the first year of the evaluation, the Hastings velocity meter was mounted too close to the value controlling the exhaust fiow and consequently did not accurately measure the flow. The valve was manualiy adjusted to maintain a negative pressure inside the glove box. The lack of reliable flow medsurements created special problems.

At the beginning of a new evaluation, the pressure drop across the air filter increased rapidly from 0.025 to $0.249 \mathrm{kPa}$ and remained at this level for nearly four months. During this time, we neither cleaned the filter medium nor removed it from the filter housing for weighing. We had assumed that an adequate flow was still passing through the filter because the glove box vacuum was within the normal limits. Although the Hastings velocity meter had indicated a very low exhaust flow, we had discourited these measurements because independent measurements showed the meter was inaccurate. We believed an equilibrium condition had been created with dust flaking off the filter surface as new dust was collected. The observation of dust accurnulation at the base of the filter gave additional weight to this hypothesis. In an effort to verify this, we made a series of flow measurements using calibrated orifices that showed our hypothesis ras wrong. The exhaust flow had been nearly reduced to zero.

Once we realized that the clogged filter was choking off the exhaust flow, we changed the procedure by routinely cleaning the filter every two weeks. This procedure was used to evaluate the electric air filter over an eightmonth period. Figure 101 shows the relative flow and the pressure drop across the filter taken during a portion of this evaluation. Note that whenever the pressure drop decraases, the flow increases. The three Cs indicate the point at which the filter was cleaned. After each filter cleaning, the pressure drop was very low and then gradually increased to its limiting value within four working days. In a similar fashion, the relative flow was high after the filter cleaning and gradually decreases as the filter resistance increased. 


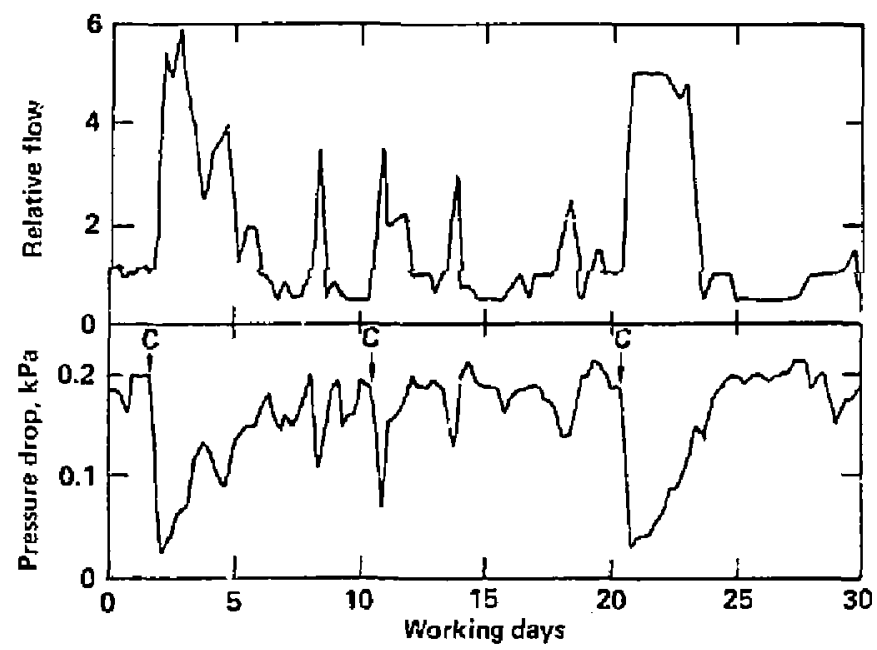

Fig. 10] Pressure drop and flow rate for electric air filter operated at variable flow rates with regular clearings. 
Figure 101 also shows a filter cleaning that had a very short-term effect on the pressure drop and flow. Presumably, the filter was improperly cleaned. Note that there are also a number of spikes in the pressure drop and flow curves that suggest changes in the particle cake structure. These spikes probably occur when some deposit flakes off the filter surface.

The above procedure provided us with sufficient information on the extension of HEPA service iife. In addition, the procedure was very simple and representative of a typical filter maintenance program. Unfortunately, the large fluctuations in the exhaust flow velocity made the theoretical interpretation of the test results very difficult. Since the exhaust flow has such a dramatic effect on the filter efficiency, we decided to change the procedure and mainusin a constant flow throughout the evaluation. We were able to maintain a constant flow of $2.41 / \mathrm{s}(5 \mathrm{cfm})$ by either closing or opening the valve when the electric air filter had either a low or high pressure drof. However, before this procedure was used, we installed a new velocity probe sufficiently far downstream from the butterfly valve to prevent interference. After installation, we callbrated the velocity probe with orifice plates.

Figure 102 shows the resulting flow and pressure drop across the fitter using the new procedure. In order to maintain a constant flow as the resistance of the prefilter increased, the operator gradually opened the valve. Whenever the flow dropped belou $2.41 / \mathrm{s}$ with the butterfly valve wide spen, the filter medium was removed from the filter holder and the particle deposits shaken off. After cleaning, the medium was placed back into service and the butterfly valve closed to maintain the constant flow of $2.41 / 5$. Figure 102 shows the relatively constant flow and the large oscillations in the pressure drop that were obtained using this procedure. The high prassure drop ard low flow during the first seven days were part of the previous evaluation. As before, the Cs represent filter cleanings. Figure 102 alss shows a break in the curves after seven days that represents the installation of a new filter medium. Although the old medium was still in good condition after 12 months of service, we wanted to begin the new evaluation procedure with a fresh filter.

Ouring the evaluation, we experienced leaks in the Gelman Model-110? filter hoiders that prevented us from measuring filter efficiencies. This became a major problem. We had used the $25-\mathrm{mm}$ plastic filter holders for 


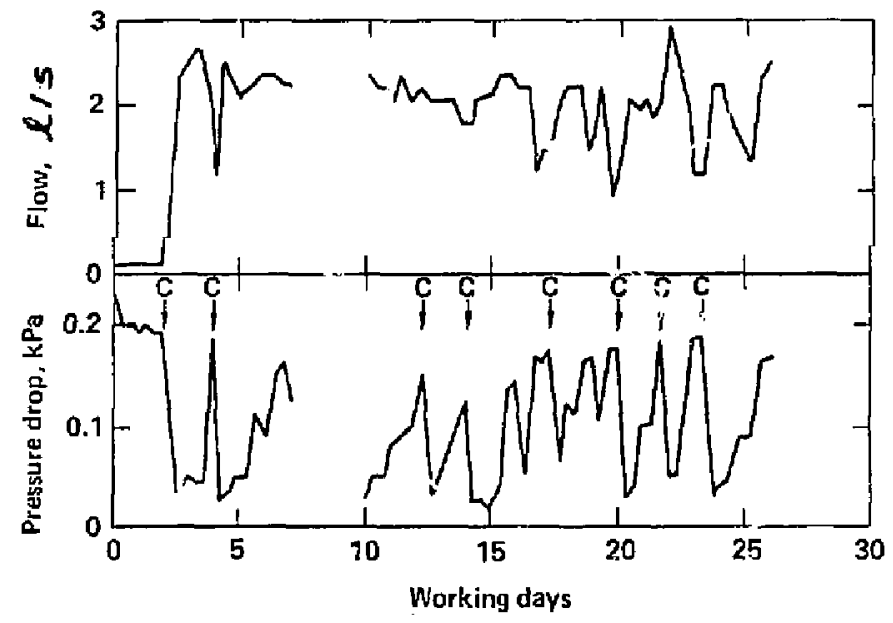

Fig. 102 Pressure drop and flow rate for electric air filter operated at constant flow with irreguiar cleanings. 
sampling aerosol cancentration before and after the electric air filter because flastic filter holders were more econumical than stainless steel or aluminum filter holders since, after each use, the filtei holder had to be decontaminated or discarded. We discovered that the Gelman filter holders leaked. As part of an established procedure, we had routinely chcaked fo. leaks prior to installing the plastic filter holders inside the sampling chamber. During these tests, we found that the majority of our filter holders leaked significantly. On a number of occasions, we spent an entire day searching, without success, for leak-free filter holders from a supply of over 100. Using Teílon tape on all of the threaded portions of the tilter holder appeared to help at times, but the holders still leaked when installed.

We tested in-line plast: filter holders from five other manufacturers and faund that they all leaked. Since we would not take filter samples of the Pu salt aerosols using leaky filter holders, we could not obtain sufficient prefilter efficiency measurements to make a quancitative comparison filter performance with and without electrification for the Pu sait aersols.

The final proolem encountered during the field evaluation was an intermittent failure of the safety interlock system that shut off the high voltage when the hinged doors were not pronnrly closed. The reed switches imbedded in the filter frame were sensitive to the postiun of the magnets nounted in the hinged doors. After constant use over many mo iths, the reed switches and inagnets were not properly aligned and would discornect the high voltage. We had solved this prob'ew in the final stages of the evaluation wher. re repiaced the high voltage power supply with a much smaller unit. Since the new power supply was not capable of delivering a potentially hazardous current, the interlock system was no longer required. The electric air filter functioned perfectly after the new power supply was installed.

Recovery of Radioactive Material. One of the important aspects of our evaluation was the recovery of radioactive materiat. Every time we cleaned the filter to increase its service life, we also recovered radioactive material. We weighed the filter before and after cleaning and determined the amount of material recovered. The average of a large number of cleanings showed that about $80 \%$ of the particle deposit within the filter nedium recovered. However, the deposit that remained after the medium was cleaned was relativeiy constant during the evaluation. Once the medium was in service for a short time, nearly $100 \%$ of the new deposit was recovered. Based an our measurements, we estimated 
that we recovered $98 \%$ of the totil dust that would normally escape to the exhaust.

HEPA Service Life. The most important finding of our field evaluation was the large extension in the HEPA service life. The electric air filter and a fresh HEPA filter were installed in the glove box at the beginning of the ewaluation in Janauary, 1979. The electric air filter was operated continuously under high voltage until the unit was removed in March, 1980. During this 14-month evaluation, we recovered over $98 \%$ of the Pu salts and saw r.o measurable increase in the 12-Pa pressure drop across the HEPA filtor. Prior to installing the electric air filter, the HEPA iilter had to be changed every month due to plugging.

From the beginning of our evaluation ir January, 1979 to September, 1983, we have used two HEPA filters in the glove box. The first HEPA filter was protected by our electric air filter for 14 months and showed no increase in pressure drop from its initial value of $12 \mathrm{~Pa}$. The second HEPA filter was protected by mechanical prefilters: a Waterweb filter for the first 18 months and then our non-electric prefilter fo the next 14 months. During these 32 manths, the pressure drop across the second HEPA filter had increased from $12 \mathrm{~Pa}$ to $50 \mathrm{~Pa}$. The mechanical prefilter was then converted to electrical operation in December, 1982 and has continued to protect the HEPA filter during the last 10 months. During this last period, the pressure drop across the HEPA fijter has increased from $50 \mathrm{~Pa}$ to $100 \mathrm{~Pa}$. It is clear that the HEPA filter is nearly plugged after 42 months of service. Since the HEPA filter would normally plug within one month, the prefilters have extended the HEPA life by greater than 42 times.

Because of this very significant extension in HEPA Iife described above, our electric air filter has become an integrai part of the Rocky Flats' production facility. Our prefitter not only saves on HEPA filter replacement, but also allows nearly $100 \%$ recovery of the Pu dust.

\section{Cost/Benefit Analysis}

We analyzed the cost/benefit of the hinged door-filter using data obtained during the field evalation in the Pu-dust vacuum box. Unfortunately, because of the problem with leaking filter holders, we were not able to measure filter efficiency as a function of particle mass loading for the electric and 
mechanical prefilter for the Pu salt aerosols as we had done for the $\mathrm{UO}_{2}$ aerosols. This data would have been used to determine the average filter efficiency during one loading cycle, an important variable in the cost/benefit analysis. In spite of this lack of data, the average filter efficiency could be determined from the efficiency medsurements in Table 12 and the trend in fi]ter efficiency during particle loading shown earlier. From these data, we determined that the average efficiency for the electric air filter over a $12-9$ loading cycle was $99.9 \%$, and $99.5 \%$ for the mechanical prefilter. Therefore, the values of the filter penetration $\left(P_{F}\right)$ to be used in the cost/benefit analysis are 0.001 for the electric air filter and 0.005 for the mechanical prefijter.

The values listed in Table 13 were used to compute the cost, volume of filter waste, material recovered and the HEPA life for various filtration systems. As before, we neglected the labor cost and the cost for cleaning the prefilter and the value of the material recovered.

Our results are shown in Table 14 for the five filtration systems: HEPA filter, HEPA filter-mechanical prefilter syten with and without prefilter cleaning, and the HEPA filter-electric prefilter system with and without prefilter cleaning.

The general trends seen in Table 14 are the same as previously seen for the $\mathrm{UO}_{2}$ evaluation. However, because of the higher efficiency of the electric air filters or prefilters, the results were more dramatic. The total annual filtration cost decreased very rapidly from $\$ 297$ to $\$ 7.90$, and finatiy to $\$ 2.51$ when we used a mechanical and electric prefilter. As previausly noted, cleaning the prefilter had very little effect on the cost of the system due to the insignificant cost of the prefilter media. In contrast to the cost, the volume of filter waste decreased more slowly from 69.2 liters to 14.7 1iters and 13.4 11ters as the mechanical and electric prefilters are added. Either cleaning or compressing the electric air filter significantly reduced the volume of fj]ter waste. Compressing the filter after cleaning reduced the waste volume by only a small amount. Table 14 also shows that the only recovery of material occured when the prefilter was cleaned and was about the same for both mechanfcal and electric prefilters. In these calculations, we assumed that the HEPA filter cannot be cleaned.

The last criterion listed in Table 14 is the HEPA filter life. Note that the HEPA life 15 inversely proportional to the cost of the filtration system 
Table 13. Values of variables used in cost/benefit analysis of electric air filters in Pu glove box with ninged-door design.

\begin{tabular}{|c|c|c|c|c|}
\hline \multirow[b]{2}{*}{ Variable } & \multicolumn{2}{|c|}{ Prefilter a } & \multirow{2}{*}{$\begin{array}{l}\text { HEPA } \\
\text { after air filter }\end{array}$} & \multirow{2}{*}{$\begin{array}{l}\text { HEPA } \\
\text { alone }\end{array}$} \\
\hline & Mechanical & Electrical & & \\
\hline$C_{P F}, C_{P H}$ & $\$ 0.02$ & 60.02 & $\$ 27.00$ & $\$ 27.00$ \\
\hline$V_{F}, V_{H}$ & 0.211 & 0.211 & 6.291 & 6.291 \\
\hline$P_{F}, P_{H}$ & $\begin{array}{l}0.005 \\
750 \mathrm{~g}\end{array}$ & $\begin{array}{l}0.001 \\
750 \mathrm{~g}\end{array}$ & $\begin{array}{l}0.00 \\
750 \mathrm{~g}\end{array}$ & $\begin{array}{l}0.00 \\
750 \mathrm{~g}\end{array}$ \\
\hline$\frac{M}{M P_{F}}$ & -- & --- & $3.7 \mathrm{~g}, 0.7 \mathrm{~g}^{\mathrm{c}}$ & $750 \mathrm{~g}$ \\
\hline$M\left(1-P_{F}\right), M\left(1-P_{H}\right)$ & $746.3 \mathrm{~g}$ & $749.3 \mathrm{~g}$ & $\cdots$ & -- \\
\hline$r_{F}, r_{H}$ & $1.75 \mu \mathrm{m}$ & $1.75 \mu \mathrm{m}$ & $0.25 \mu \mathrm{m}$ & $1.75 \mu \mathrm{m}$ \\
\hline$K_{F} r_{F}, K_{H}, r_{H}$ & $12 \mathrm{~g}$ & $12 \mathrm{~g}$ & $15 \mathrm{~g}$ & $69 \mathrm{~g}$ \\
\hline f & 0.98 & 0.98 & 0 & 0 \\
\hline g & 0.16 & 0.16 & $0.45^{d}$ & $0.45^{d}$ \\
\hline
\end{tabular}

a variable subscript is $F$.

b variable subscript is $H$.

$c$ dust load on the HEPA is 3.7 g using the mechanical prefilter and 0.7 ? with the electrical prefilter.

if the wooden frames are burned then the volume reduction fracticn is 0.11 .

Table 14. Calculated annual performance of the hinged-door glove-box filtration systems based in Pu-dust vacuum box.

\begin{tabular}{|c|c|c|c|c|}
\hline \multirow[b]{2}{*}{$\begin{array}{l}\text { Filtration } \\
\text { system }\end{array}$} & \multirow[b]{2}{*}{$\begin{array}{l}\text { Fijter } \\
\text { cost }\end{array}$} & \multicolumn{3}{|c|}{ Performance Criteria } \\
\hline & & $\begin{array}{l}\text { Volume of waste } \\
\text { (with compression) }\end{array}$ & $\begin{array}{l}\text { Material } \\
\text { recovered } \\
750 \mathrm{~g} \text { input }\end{array}$ & $\begin{array}{l}\text { HEPA } 1 \text { ife } \\
\text { (11/year) }\end{array}$ \\
\hline HEPA & $\$ 297.00$ & 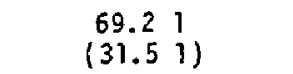 & $0 \mathrm{~g}$ & $1 x$ \\
\hline $\begin{array}{l}\text { HEPA plus mechanical } \\
\text { prefilter }(n=1)\end{array}$ & 97.90 & 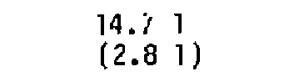 & $0 \mathrm{~g}$ & $28.6 x$ \\
\hline $\begin{array}{l}\text { HEPA plus mechanical } \\
\text { prefilter }(n=62)\end{array}$ & 96.68 & $\begin{array}{l}1.761 \\
(0.731)\end{array}$ & $735 \mathrm{~g}$ & $28.6 \times$ \\
\hline $\begin{array}{l}\text { HEPA plus electric } \\
\text { prefilter }(n=1)\end{array}$ & $\$ 2.51$ & $\begin{array}{l}13.41 \\
(2.231)\end{array}$ & $0 \mathrm{~g}$ & $142.9 \times$ \\
\hline $\begin{array}{l}\text { HEPA plus electric } \\
\text { prefilter }(i=62)\end{array}$ & $\$ 1.28$ & $\begin{array}{c}0.501 \\
(0.261)\end{array}$ & و 735 & $142.9 \times$ \\
\hline
\end{tabular}


and increases to 28.6 and 142.9 times the original HEPA life as a mechanical and an electric air filter are added, respectively. Table 14 illustrates well the major conclusion of our cost/benefit study that a high-efficiency prefilter which can be cleaned many times will result in the most cost-effective system.

We verified the validity of the calculated performance shown in Table 14 in a 56-month evaluation. During the first 14 moiths, an electric prefilter was used to protect the HEPA filter. Since the HEPA filter had shown no increase in pressure drop, the extension of HEPA life due to the electric prefilter is much greater than the actual 14 months. This test also showed that $98 \%$ of the collected dust could be recovered by shaking the filter media. A total of three prefilter media were used in this evaluation, with one medium 1 asting for 12 months. The other two did not have to be replaced, but were changed to coincide with changes in the test procedure.

(start correx here)

The results of the annual performance of the prefilter systems are shown in Table 15. Please note that the data for the HEPA plus electric prefilter were determined after terminating the evaluation at 14 months. However, since the same pressure drop across the HEPA filter remained constant at $12.5 \mathrm{~Pa}$, the values for the filter cost, volume of waste, and HEPA life in Table 15 would approach the values shown in Table 14 if the evaluations were continued unt il the HEPA filter clogged.

Table 15 also shows the performance value of a prefilter-HEPA filter system evaluated over 42 months. Although the evaluation was a mixed evaluation using a mechanical prefilter during the first 32 months and an electric prefilter during the last 10 months, it showed that extremely large extensions in HEPA life are achieved even with mechanical prefilters. Since prefilters are generally not used in the exhaust of most glove boxes, our finding shows that even mechanical prefilters will significantly extend the life of HEPA filters. Unfortunately insufficient data was obtained to show the major differences in HEPA life predicted in Table 14 for mechanical and electric prefitiers. 
Table 15. Measured anilual performance of giove-box filtration systems in Pu-dust vacuum bax.

\begin{tabular}{|c|c|c|c|c|}
\hline \multirow[b]{2}{*}{$\begin{array}{c}\text { Filtration } \\
\text { system } \\
\end{array}$} & \multirow[b]{2}{*}{$\begin{array}{l}\text { Filter } \\
\text { cost }\end{array}$} & \multicolumn{3}{|c|}{ Annual performance criteria } \\
\hline & & volume of waste & $\begin{array}{l}\text { Material } \\
\text { recovered } \\
\text { (750 g input) }\end{array}$ & $\begin{array}{l}\text { HEPA Tife } \\
\text { (12/year) }\end{array}$ \\
\hline HEPA (12) & $\$ 324.00$ & 75.51 & 0 & $3 x$ \\
\hline $\begin{array}{l}\text { HEPA plus } \\
\text { electric } \\
\text { prefilter } \\
\text { (0.86 HEPA } \\
3 \text { prefilters) }\end{array}$ & $\$ 23.28^{a}$ & $6.01^{a}$ & $735^{\mathrm{b}}$ & $14 x^{a}$ \\
\hline $\begin{array}{l}\text { HEPA plus } \\
\text { various } \\
\text { prefilters } \\
\text { (0.29 HEPA } \\
7 \text { prefilters) }\end{array}$ & $\$ 7.97^{\mathrm{C}}$ & $3.3 \mathrm{j}^{\mathrm{C}}$ & $735^{6}$ & $42 \times$ \\
\hline
\end{tabular}

\footnotetext{
a Assuming HEPA filter plugged, but pressure drop never increased.

b Calculated from actual material recovered.

c Waterweb prefilter not included.
} 


\section{ELECTRIC AIR FILTERS FOR VENTILATION SYSTEMS}

\section{INTROOUCTION}

We designed two experimental electric air filters for use in nuclear ventilation systems that act as prefilters for the HEPA filters in such systems. Both units have the overall dimensions of a standard HEPA filter in which the filter media is pleated to increase the filter surface area. These "prefilters" significantly extend the life of the HEPA filters located downstream and reduce the overall cost and radioactive waste of the prefilter-HEPA fiiter system.

Since the cost of filter replacement is a major fraction of the total filtration cost in a nuclear facility, the use of a prefilter, whether conventional on electrified, is cost-effective only when the replacement or cleaning cost for the prefilter is much lower than simply replacing the HEFA filter. A critical factor controlling replacement or cleaning cost is the filter housing. The housing in which the prefilter is installed must have provisions for easily replacing or cleaning the prefilter. Filter housings for prefilters historically have had the same basic design as used for HEPA filters, therefore, replacing a prefilter in these housings required about the same effort as replacing a HEPA filter. It is then not surprising that prefilters have not been widely used in nuclear ventilation systems.

We recognized the importance of a properly designed prefilter housing and designed our electric air filters and filter housing as an integrated prefiltration system requiring minimal maintenance. Figure 103 shows one of our experimental prefilters mounted inside a ventflation housing designed for minimizing prefitration maintenance. To increas? the flexibility of our evaluation program, we bujit two separate filter housings into a single unit and joined the inlet and exhaust openings into a common duct. This ventilation system permited us considerable flexibility in our field evaluations. We simultaneously evaluated two prefilters or a prefilter-HEPA filter under the same conditions and diverted the air flow from one chamber to the other. The primary features of the ventilation housing are the glove ports and entry-exit ports which permited prefilter replacement or cleaning without disruption of the ventilation flow. We have essentially built a glove box around the prefilter to allow in situ maintenance. 


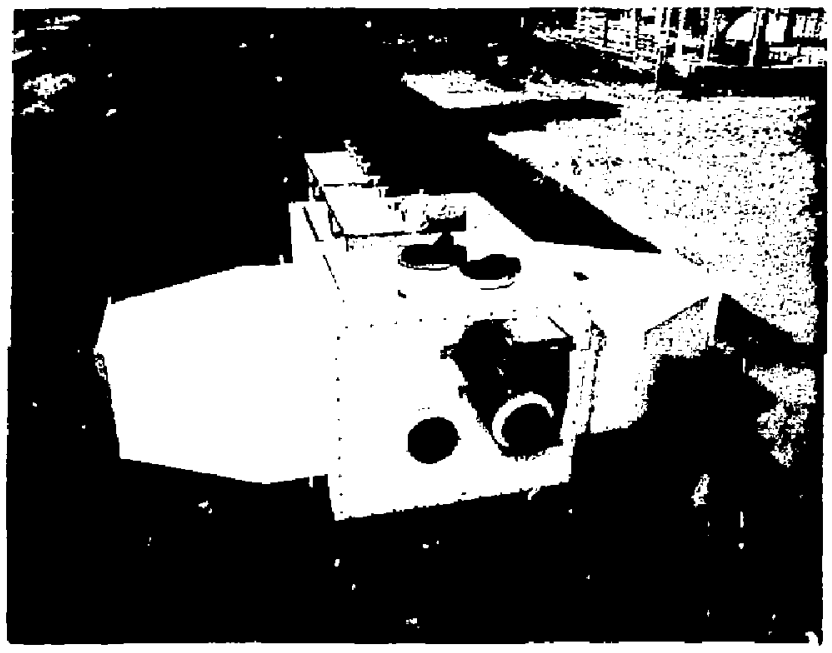

Fig. 103 Low-maintenance ventilation housing for electric air filters - HEPA filter penetration system. 
Design

One version of our prefilter has the prefilter as a permanent component of the ventilation system. It has a rigid, stainless-steel frame and durable high voltage and ground electrodes. This prefilter design, shown in Fig. 104, uses an inexpensive filter medium inserted into the filter unit whicl, is removed when it becomes plugged. The filter medium is sandwiched between the high-voltage and ground electrodes pleated to increase the filter surface area. The front ground electrodes can be pulled out of the filter unit to provide access to the filter medium.

Our earlier design was an electrified version of a commercfally available filter which had a replaceable filter medium mounted between a rigid supporting frame and a removable front screen. This proved to be clumsy because of the bulk of the unit. We made the filter more accessible by attaching the front electrode to a pivot connection on slides mounted on the frame. This allowed the front electrode to be pulled out of the filter housing and rotated for eificient media replacement. (Replacing filter media is a serious prablem only in radioactive environments.)

\section{Laboratory Evaluation}

We evaluated ihis electric prefilter in our large-scale filter test facility as a function of applied voltage at $0.47 \mathrm{~m}^{3} / \mathrm{s}$ (1000 cfm) using $\mathrm{NaCl}$ aerosols.

Figure 105 shows the efficiency using three different grades of filter media. The filter media differ primarily in the fiber diameter which decreased in sequence for the AF-18, AF-4 and AF-3 media. It is well-established that filter media with smalier fiber diameters have higher efficiencies and pressure drops than medla with larger fiber diameters. ${ }^{(80)}$ This accounts for the increase in pressure drop and zero-voltage efficiency for the sequence AF-18, AF-4 and AF-3. As $10 \mathrm{kV}$ is applied to the high-valtage electrode, filter efficiency increases from $38.1 \%$ to $90.1 \%$ for the AF-18 medium, from $85.0 \%$ to $97.7 \%$ for the Ar .4 medium and from $95.4 \%$ to $97.9 \%$ for the AF-3 medium. The corresponding pressure drops for the $A F-18$, 


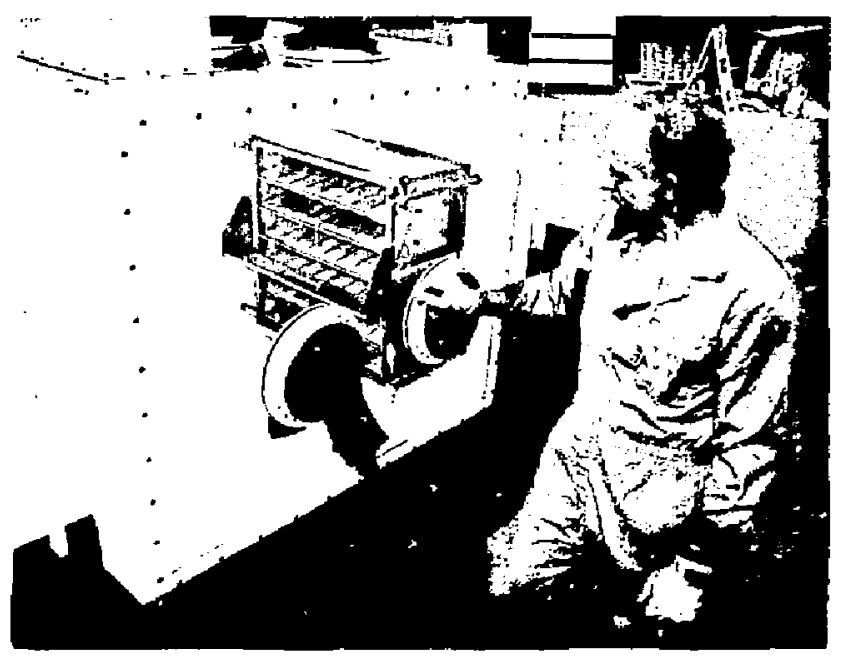

rig. 104 Electric ventilation filter designed as permanent component of the ventilation duct using permanent electrodes and replaceable filter media.

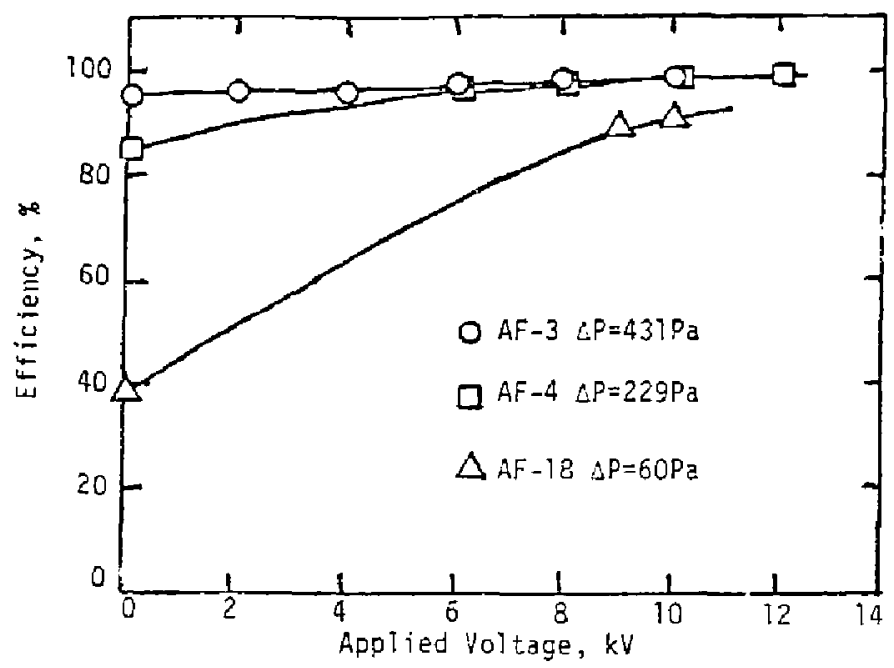

Fig. 105 Filter efficiency of the electric ventilation filter design having permanent electrodes as a function of applied voltage for different filter media. 
$\mathrm{AF}-4$ and AF-3 media are $60 \mathrm{~Pa}, 229 / \mathrm{Pa}$, and $431 \mathrm{~Pa}$, respectively. Based on our maximum resistance criterion of $250 \mathrm{~Pa}$, the $\mathrm{AF}-3$ medium could not be used in our experimental filter.

\section{Cost/Benefit Analysis}

The electric filter design using permanent electrodes was intended for high-particulate applications where the frequency of filter changes would be high. The total filtration cost is the sum of the fixed capital cost plus the operating cost. In applications of high dust loadings, the operating cost of replacing the filter is high and a design that minimizes this cost is preferred. The cotal filtration cost can be determined from the filter used as a prefilter to a riPA filter and from the filter used alone. In both rases, a high purchase cost for the filter, $C_{P F}$, increases the operating cost. For our filter design, the purchase cost is low for the relatively inexpensive filter media used in the electric filter.

We also recognize that the labor cost for replacing the filter, $C_{L F}$, is equally important to the purchase cost. A fitter design that has a low purchase cost but a high labor cost for replacing the filter will result in a high operating cost. One of the most frequent errors made in designing a prefiltration system in the nuclear industry is neglect of the fabor costs involyed in replacing the prefilters. Prefilters are generally housed in a fixture sinilar to that used for HEPA filters which results in comparable labor costs for replacing both the HEPA filter and the prefilter. If a prefilter has to be replaced by a bag-in or bag-out operation from standard glove boxes, or from enclosed cells (or, if an operator has to enter a contaminated cell wearing a protective suit and independent air supply), then the prefilter design will generally not be cost-effective. For this reason, it is not advisable to use prefilters as the first stage of a multi-stage HEPA plenum chamber.

We reduced the labor cost for replacing the electric filters by designing a special housing for the experimenal filters as shown in Fig. 103. Glove ports allow the filter or filter media to be replaced while inside the housing. Fresh or used filter media can be conveniently added to or removed from the housing through an entry-exit port. The strategy for minimizing the labor costs in replacing the filter media in our experimental ventilation 
filters was taken from our successful experience in the glove box prefilters described in the previous section. The najor difference here is that ventilation filters are larger and require more space to replace than the glove-box prefilters. The filter housing shown in Fig. 103 also has two compartments in parallel to allow filter changes without interrupting the ventilation system. A diverter valve can direct the exhaust flow through either one or both of the compartments as needed. While one filter is being changed, the air is directed to the ather compartment to make filter changing easier and to avoid contaminating the downstream ducting.

The reduction in the labor cost for replacing the filter or filter media results in an increased capital cost due to the additional housing. A careful analysis of the total filtration cost must therefore include not only the operating cost due to the media cost, $C_{P F}$, and the labor cost, $D_{L F}$, but also the capital costs of the filter and the filter housing. Without such an analysis, it is possible that a high operating cost is merely exchanged for a high capital cost. The critical factor in this analys is is the miass dust loading, $M$, in the air to be filtered. If the dust loading is sufficiently high, then the decreased operating cost will more than compensate for the increased capital cost.

Two more important factors in the ventilation filter housing are cleaning and reusing the filter media. As we have previously shown, cleaning the filter results in a large reduction in the operating cost for a filte- having a high purchase cost, but anly a modest reduction in the operating cost for a low-cost filter. Since the filter media cost in the permanent electrode design is low, cleaning the media does not result in large cost savings. But, as previously noted, cleaning the media yields large reductions in the volume of filter waste.

If the maintenance of a ventilation fiiter involves cleaning the filter $n$ times before discarding, then the operating costs are reduced by $n$ times. This is only true if the cleaning cost, $C_{C F}$, is negligibie, as was the case for our glove-box prefilters. We minimized the cleaning cost for the ventilation filter by having the filter mounted in a special housing equipped with glove ports. The net reduction in the operating cost due to filter cleaning will tend to favor filter designs having low capital costs and high operating costs. In order for the filter design with the filter media separated from permanent electrodes to be competitive with alternative 
designs, the capital equipment cost of the electrodes and frame has to be minimized. This will be a difficult task for our filter design because of the requirements to maintain close tolerances in the spacing between the high-voltage and ground electrodes, and the need for an efficient mechanism to remove the front electrode from the filter housing.

DISPOSABLE FILTER WITH ELECTRODES INTEGRATED WITH THE MEOIA

Design

Electric ventilation filters can be designed as a disposable 1 iteris in Which the electrades are integrated with the filter media. Although the operational cost for such a design will be more than the cost for the previous design in which the filter media can separate from the electrodes, the total unit cost will be lower due to a substantially lower capital cost. The total annual filtration cost, however, depends oupon the number of filter changes which will benefit the disposable design at low dust loadings and the permanent electrode design at high dust loadings. An integrated design also has the operational advantage of decreased handing of the filter unit. The electric air filter having integrated electrodes and filter media is handled once during installation and once during removal. The electric filter design having permanent electrodes has increased handling by removal and replacement of ti'le front electrode each time the filter media is replaced. After a number of media replacements, it is possible that the electrodes may become distorted and will no longer fit into the filter housing, thereby requiring additional maintenance. Moving mechanical components, like the slide support in the permanent electrode design, are especially prone to failure in a disty environment.

We developed a disposable electric ventilation filter having the electrodes integrated with the filter media to take advantage of decreased handing and lower purchase cost. In order to meet our design criteria of low pressure drop and low air-flow velocities, the filter media and electrodes have a pleated configuration. Our initial design had two continuous, pleated electrodes with the Af -19 medium sandwiched between. Three insulated spacer bars (one in the center and one at both ends) provided the necessary structural support to maintain the shape of the front electrode. Without this support, 
the electrode behaved like aspring and popped out of the housing. Three additional insulated spacer bars were used in a similar fashion to maintain the shape of the rear electrode. Pushing in the spacer bars pressed the electrodes together and made good contact with the filter medium. A 10-mm separation was maintained between the front and rear electrodes by three strips of rubber that were attached to the rear electrode.

The major difficulty in this design was maintaining the proper distaice between the two electrodes to prevent sparking. Both of the eiectrodes distorted and buckled as the unit was assembled. Although we tried a number of different schemes to maintain the proper electrode spacing, we were only able to apply $6 \mathrm{kV}$ before sparking occurred. This voltage was insufficient for significantlv improving the filter efficiency and so we abandoned this desigri concept.

Our second design of a disposable electric ventilation filter avoided the problems of electrode sparking due to a non-uniform spacing between the electrodes. Figures 106 and 107 show the inlet and exit sides of the disposable electric prefilter. The high-voltage and ground electrodes are glued on the pleated filter medium and the filter medium glued to the square sealing frame. The electrodes were cut from a flexible stainless-steel screen. To prevent short circuiting between the high-voitage and ground electrodes at the sewn edges of the filter media, we used rectangular screen segnents rather than a continuous strip. The screen segments were cut to maintain a $3.5-i$ inch. border around the perimeter of each pleat. The ground electrode segments shown in Fig. 107 were electrically connected to the sealing frame by means of a flexible cable. Figure 106 shows an electrode bus that connects the highvaltage electrodes to a common lead. Note that high voltage is applied to the interior electrodes to prevent short circuiting with the ventilation duct in case of accidental misuse or improper installation.

Since the electrodes and sealing frame are permanently glued to the filter medium, when the filter medium is plugged, the entire electric filter must be replaced, hence its designation as a disposable prefilter. We mounted the disposable prefilter on a transparent plastic frame to allow visual inspection of the installed prefilter. In field applications, the sealing frame of the disposable filter would be mounted flush against a flange on the ventilation duet. 


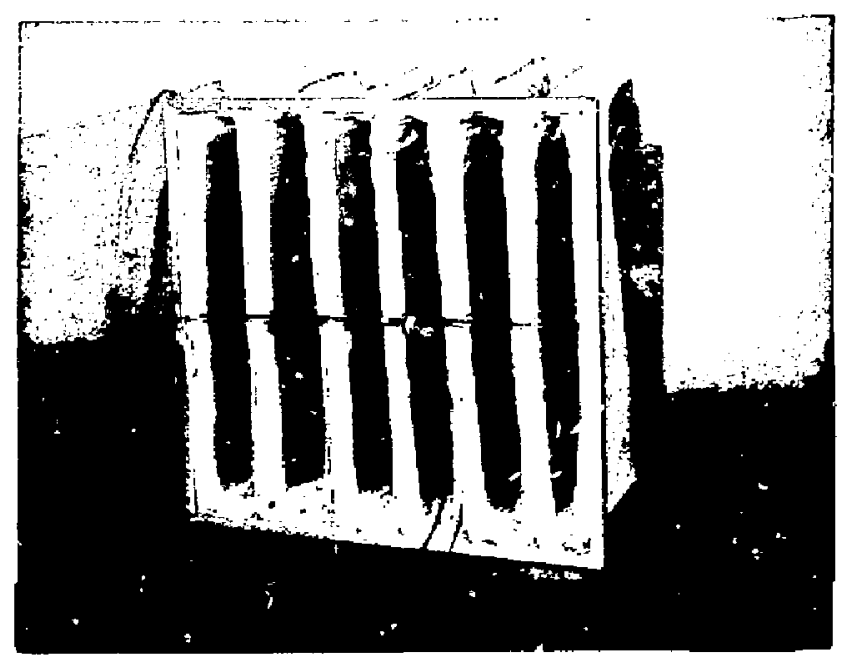

Fig. i06 Irlet side of risnosable electric ventilation filter having electrodes integrated with the fiiter media.

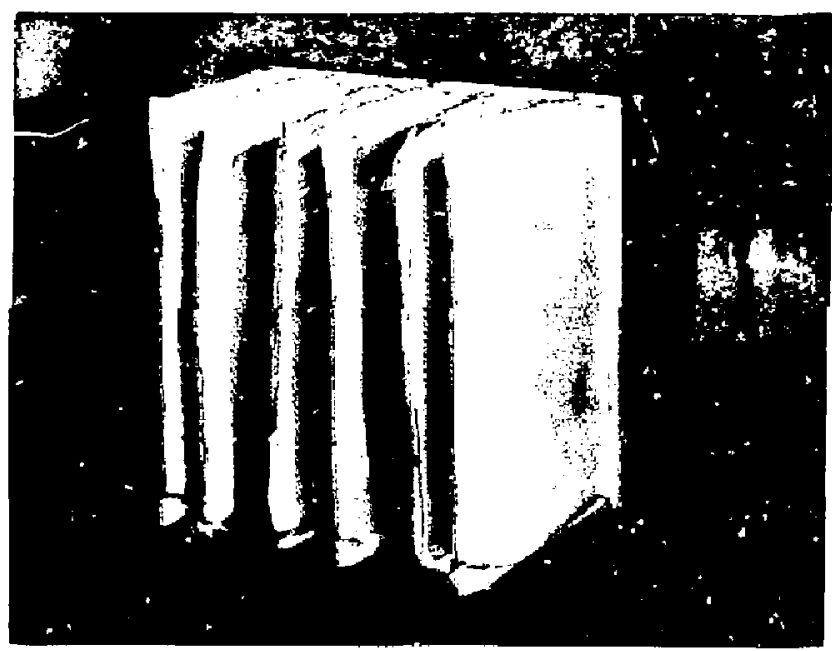

Fig. 107 Exit side of disposable electric ventilation filter having electrojes integrated with the filter media. 
The replacement of the disposable filter is easier in some respects and more difficult in others when compared to replacing the filter medium in the permanent fitter model. Mounting the disposable filter on : : sealing flange of a ventilation duct is much easier than replacing the filter medium in the permanent fijter model. However, since the disposable filter has a rigid frame and integral electrodes, the disposable fiiter is more bulky and hence more difficult to pass through the entry-exit ports. So, bagging out the disposable filter is still much easier than bagging out a HEPA filter since the disposable filter is light-weight and the pleated media can be folded to reduce the filter size.

\section{Laboratory Evaluation}

We conducted a laboratory evaluation of the disposable prefilter design in our large-scale filter test facility. Figure 108 shows the filter efficiency of the disposable prefilter design as a function of applied voltage at two air flow rates. The filter efficiencies were determined using $\mathrm{NaCl}$ aerosols generated with a Wright nebulizer and measured with a light-scattering photometer. The $\mathrm{NaCl}$ aerosols have an AMMD of $0.8 \mu \mathrm{m} \mathrm{with} \mathrm{a} \mathrm{g}=2.0$.

Figure 108 shows that the filter efficiency increases significantly with increasing voltage at both flaw rates, although the lower rate shows the greater increase.

The effect of air flow on filter efficiency and pressure drop for the disposable filter is shown in Fig. 109 which shows that higher efficiencies and Tower pressure drops are ottained at lower air flows. The two efficiency curves represent the filter efficiency with $0 \mathrm{kV}$ and $8 \mathrm{kV}$ applied to the filter electrodes. The efficiency of the electric air filter is much higher at the lower air flows because of the increased residence time that permits the electrical forces to attract particles.

Field Evaluation of Disposable Electric Air Filter in LLML's Uranium Burn Box

We selected the LLNL uranium burn box for our field evaluation because it had one of the highest rates of radioactive particulate emissions at LLNL and would therefore provide field data within a relatively short time. The uranium burn box was designed to oxidize machine turnings and chips of depleted 


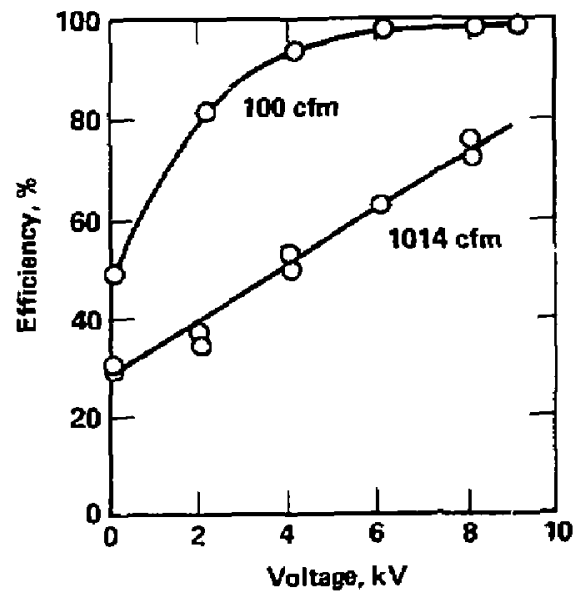

Fig. 108 Efficiency of disposable electric filter as a function of applied valtage at 100 and $1014 \mathrm{cfm}$.

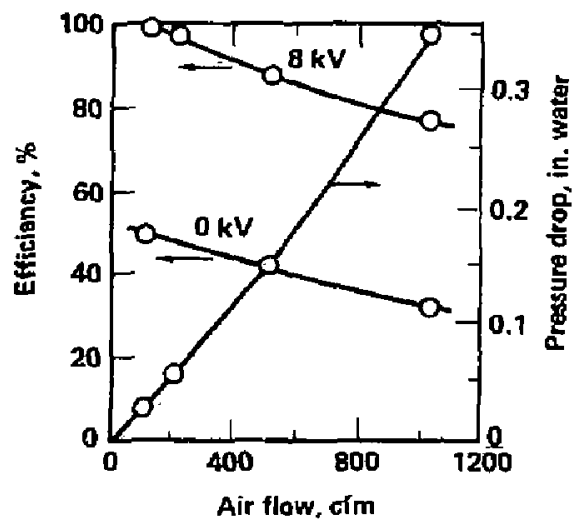

Fig. 109 Filter efficiency and pressure drop as a function of air fiow for the disposable electric prefilter. 
uranium metal into a stable form for shipment and burial. If the uranium turnings are not stabilized, they ignite and burn when exposed to oxygen. Uranium turnings are brought to the facility in barrels filled with water to prevent premature oxidation.

A barrel containing the depleted uranium metal is loaded into the left side af the burn box. The barrel is then mounted on a pivot and the lid removed. After the access door is closed, the box is purged with an argon atmosphere. The contents of the barrel are then poured into a tray located beneath the barrel that sieves out the turnings from the water/oil mixture. Since the barrels often contain siudge, the turnings are usually washed with water. The tray of uranium turnings is then moved into the center chamber of the burn box where they are ignited with an electric arc and burned $t ;$ adding oxygen. After the oxidation is complete and the tray is cooled down, the tray is transferred to a chamber where the ash is vacuumed into disposal barrels.

Each chamber has a separate exhaust and HEPA filter that connect to a common exhaust line. The exhaust from the two end chambers passes through a 500-cfm HEPA filter mounted on top of the respective chambers. A heat exchanger inside the center chamber cools the exhaust before passing through two 135-cfm HEPA filters. The exhaust from this chamber is saturated with water vapor and has an extremely high particulate loading. These severe conditions normalily cause the two $135-\mathrm{cfm}$ HEPA filters to plug after approximately six burns (three days of operation) and shut down the operation until the filters were changed. An examination of the HEPA filters showed they had a neavy particle deposit and were saturated with water.

We designed a system to remove the water vapor that added hot dilution air to the exhaust from the burn box. The dilution air increased the contaminated air to be filtered from $50 \mathrm{cfm}$ to I50-200 cfm depending on the flow restrictions.

He built and installed a special ventilation system shown in Fig. 110 for evaluating our prefilter. The ventilation system consisted of two HEPA filter housings obtained from MSA and appropriate transition aucting. A sufficient number of viewing ports were added to the transition ducts to permit visual inspection of the prefilter and HEPA filter. We also added pressure gauges to measure the pressure drop across the prefilter and HEPA filter, a hot-wire probe to measure the tot.al exhaust flow, and a light-scattering photometer to measure the efficiency of the prefitter. This diagnostic equipment enabied us to evaluate the perfornance of the prefilter on a routine basis. 


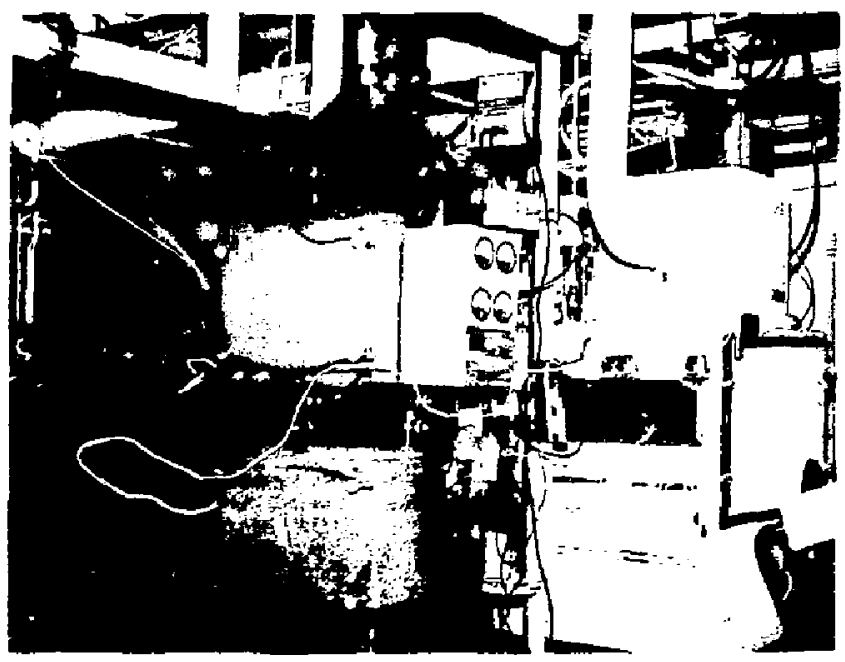

Fig. 110 Ventilation system built for evaluating the disposable electric filter. 
The disposable prefilter is mounted in a clear plastic fma to make the prefijcer compatible with the sealing mechanism of the filter housing. Before the prefilter is pushed into the filter holder, the high-voltage lead from the filter is connected to the high-voltage cable inside the housing. Figure 111 shows the prefilter installed inside the filter housing as seen through one of the viewing ports. Note the high voltage lead extending from the prefilter to a throughput on the ducting wall. The horizontal rod in the photograph is the upstream aerosol sample line that can traverse the width of the filter housing.

Results. We then monitored the performance of the electric prefilter during the uranium burns. Figure 112 shows the filter efficiency and pressure drop of the prefilter as a function of actual burning time for one fijter loading cycle. The seven hours of total burn time represents six separate burns. The electric filter had $8 \mathrm{kV}$ applied to the electrodes for most of the evaluation with. short intervals at zero voltage. The avel age efficiency was $98 \%$ at $8 \mathrm{kV}$ and $92 \%$ at $0 \mathrm{kV}$. We do not know the cause of the large $\therefore$ iuctuations seen for both the mechanical and the electric filters. They were not caused by measurement errors since we were able to repeat efficiency measurements over a relatively short time interval.

We encountered two p.oblems during the evaluation. The first prob?em was due to excessive humidity at the beginniry oi each burn that would occasionally saturate the filter media and cause the high-voltage power supply to shut of $f$ due to the overload. However, after a few minutes into the burn, the humidity was sufficiently reduced and the power supply functioned normally. The second problem was an electrical overload condition caused by the particle deposits. This probiem became noticeable taward the end of the filter loading cycle and even prevented the high voltage from being turned on during the last burn. A visual inspection of the filter showed that the uranium aerosols had filled the bottom portion of the bag. We speculate that the weight of the dense uranium dust had altered the electrode separation by the increased gravitational force. The prefilter was then removed from the ventilation housing.

\section{Cost/Benef it minalysis}

The design of the prefilter ventilation system in the uranium burn box provides an excelient example of why prefitters are rarely used in the nuclear industry for removing radioactive particles. Although we had used a standard 


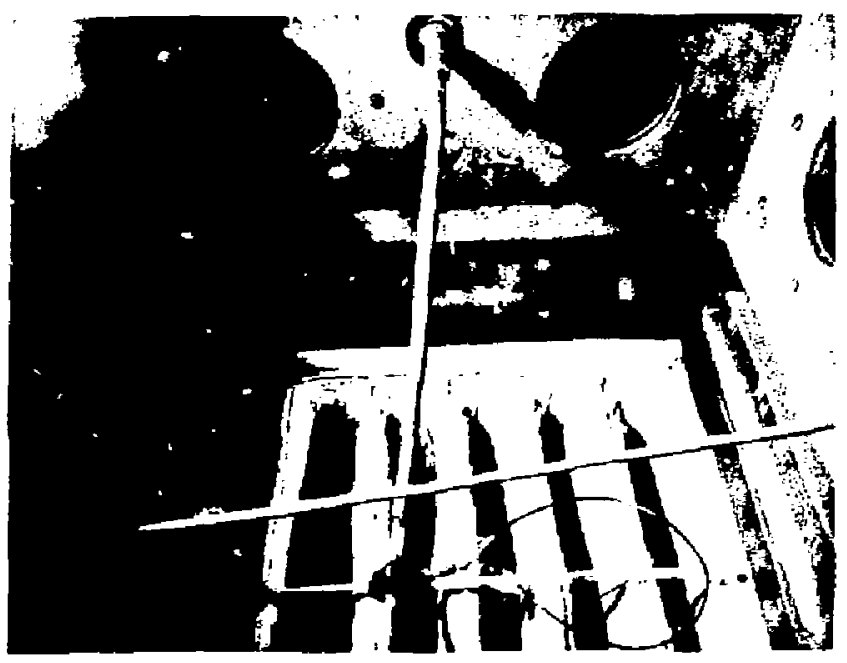

Fig. 11 Disposable prefilter installed in the filter housing.

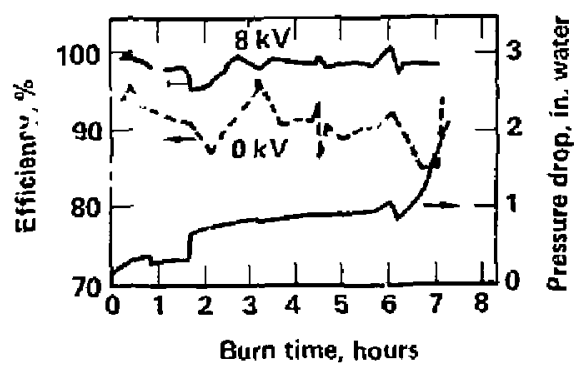

Fig. 112 Performance of aisposable electric filter in uranium burn box suring one loading cycle. 
nuclear grade filter housing for the prefilter, our evaluation for anly one loading cycle of the prefilter showed that the ventilation system is not cost-effective. After the prefilter had become plugged, the ventilation system was shut down and the prefilter removed with as much difficulty as a HEPA filter. Cleaning the prefilter and reinstalling it in the ventilation duct would add mare to the filtration cost than discarding the used filter and installing a fresh filter.

However, a rather smali modification to the exhaust system can change the prefilter installation from an uneconomical system to a very cost-effective system. The exhaust from the uranium burn box entered the preiliter housing from the top. In order to clean the prefilter, it had to first ie removed from the ventilation system. However, if the exhaust from the uranium burn box entered the ventilation system from the bottom and flowed up, the prefilter could be cleaned in situ with a minimal effort. Deposits on the prefilter could be removed by using an automatic or manual shaker or vibrator that would knock the deposits from the filter and drop them into a hopper below. A single prefilter could then be used over a large number of loading cycles instead of only one. He cannot give a quantitative estimation of the Hi.PA life extension and the total filter costs and volume of filter waste because wa do not have sufficient baseline data on the HEPA life in the uranium burn box, but, based on the average prefilter efficiency of $98 \%$, and the values of particle loading ill HEPA filters used in tie $\mathrm{UO}_{2}$ powder-handling box, we estimate that the HEPA life could be extended by more than 10 times.

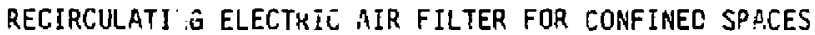

\section{INTRODUCTIOAN}

Recirculating air filters have become very gopular in recent years to remove particulates from industrial work environments because of the substallial savings in heating and cooiing costs. Portable rnom $e^{i}$ i :ieaners that recirculate filtered air irso the room have also become widei. sad in office and factory environnenis. These portable recirculating air ititers supplement the existing ventilation system. The key difference between a conventional air filter and a recirculating air filter is that the 
recirculating air filter discharges its exhaust into the same volume that is being filtered white the conventional air filter discharges its exhaust into another volume. The same filter element may be used in either application.

Recirculating air filters are especially cost-effective in the nuclear industry when an existing ventilation system may be inadequate. The cost of retrofitting a higher capacity air-cleaning syster to an existing facility is so high that $j$ is generally more economical to accept incicased maintenance costs due to the high particulate levels than to reduce the particulate levels. A good example of this situation is provided by the ${ }^{238} \mathrm{PuO}_{2}$ fuelfabrication facility at the Savannah River Plant, South Carolina. The approximate annual maintenance cost is $\$ 250,000$ due to suspended particles in their fabrication cells. ${ }^{65}$ Table 16 itemizes the maintenance costs in terms of the replacement cast for the iteris used in the fabrication cells.

Details of the operation and costs are described elsewhere ${ }^{65}$. The use of a recirculating air filter in this facility would result in a major reductiun in the operating costs. We est imate that a theoretica? reduction of $100 \%$ of the suspended particles will result in an annual savings of $\$ 245,250$ in operational costs, not including the burial cost of high-level radioactive waste. A realistic reduction of $70 \%$ of the suspended particles will yield an annual savings of $\$ 172,000$.

We developed a recirculating riectric air filter for evaluation at the Savannah River Plart to demonstrate the use of electric air filters in another application. Our other experimental filters were designed for existing ventilaxion systems as prefilters to HEPA filters. The experimental filter for the Sivannah River evaluation is a self-contained unit that is independent of the existing ventilation system. As such, it avoids the costly retrofit and severe design constraints when installed in ventilation systems. The filter is a self-contained unit that recirculates contaminated air from a confined space through an electric filier and blower and then exhausts back into the same confined space. The severe particulate problem in the fue 238 fabrication facility also represents : formidable challenge to our experimental electric air filter. The severity of the particulate problem is nat only due to the relatively high concentration of suspended particles, but also to the extremely high radioactivity of the particles. The specific activity of the ${ }^{238} \mathrm{Pu}$ aerosols is over 200 times greater than that encountered in previous fleld evaluations. Another reason for the severity of the problem is the small 
Table 1C. Total annual replacement costs of Savannah River fuel-fabrication plant.

\begin{tabular}{lccc}
\hline Item & Present cost & $\begin{array}{l}\text { Cost with 100\% } \\
\text { particulate control }\end{array}$ & $\begin{array}{l}\text { Potential } \\
\text { savings }\end{array}$ \\
\hline Manipulators & $\$ 185,640$ & $\$ 38,205$ & $\$ 147,435$ \\
Gloves & $\$ 82,459$ & $\$ 2,829$ & $\$ 79,630$ \\
Hoists & $\$ 17,232$ & $\$ 930$ & $\$ 10,302$ \\
HEPA filter & $\$ 8,801$ & $\$ 918$ & $\$ 3,883$ \\
$\quad$ TOTAL & $\$ 288,132$ & $\$ 42,882$ & $\$ 245,250$ \\
\hline
\end{tabular}


size of the suspended particles. Measurements of the particle-size distribution with a Coulter Counter indicated that most of the particles fall below the instrument detectability of $0.5 \mathrm{wm}$ diameter. ${ }^{65}$ The use of electric filters is better than mechanical filters for such aeros.js since electric filters dramaticalyy improve collection efficiency for particles below $1.0 \mathrm{um}$. We have demons'rated previously in laboratory experiments that increased filter efficiency for electric filters is most pronounced for submicron particies where mecharical filters have their lowest efficiency. Electrification increases efficiency from 15-85\% for 0.2um-diameter particles (at larger particle sizes). 'observe a smaller increase in efficiency due to electrification because the mechanical efficielicy increases dramatically. A successful demonstration would prove the credibility of the electric air filter as a practical air-cleaning device.

FUNGAMENTAL CONCEPTS OF RECIRCULATING AIR FILTERS

The concepts of air cleaning using recirculating air filters differ significantly from those in conventional air-cleaning sytems in the nuclear industry. The recirculating air filter is normally contained within a rom or chamber which has its own external ventilation system. With the external ventilation system in operation, only a portion of the chamber air volume is processed by the recirculating filter, thus imposing a limitation on its cleansing ability even if it possesses a $100 \%$ efficiency for particulate removal. In order to assess this 1 imitation, we must consider two operating environments which the recirculating filter might encounter: (1) a continuous, constant aerosol source, and (2) no aerosol source.

To under itand the behavior of a recirculating filter in a chamber having a constant aerosol formation, consider the aerosol sour.es and sinks in the example illustrated in Fig. :13. The chamber has a $V$ volume and a ventilation volumetric flow rate of $F_{1}$. The aerosol source is assumed to enter the chamber having a concentration $C_{0}$. In actual situations, the aerosol source would more likely te within tha chamber itself. Ir any case, for this simplified analysis, we are assuming a thorough mixing of the aerosols within the chamber volume. The chamber ventilation exhaust supplies an aerosol sink of $C F_{1} / V$ wtere $C$ is the chamber concentration at any time, $t$. The recirculating filter in the chamber supplies a similar sink term where $F_{2}$ is 


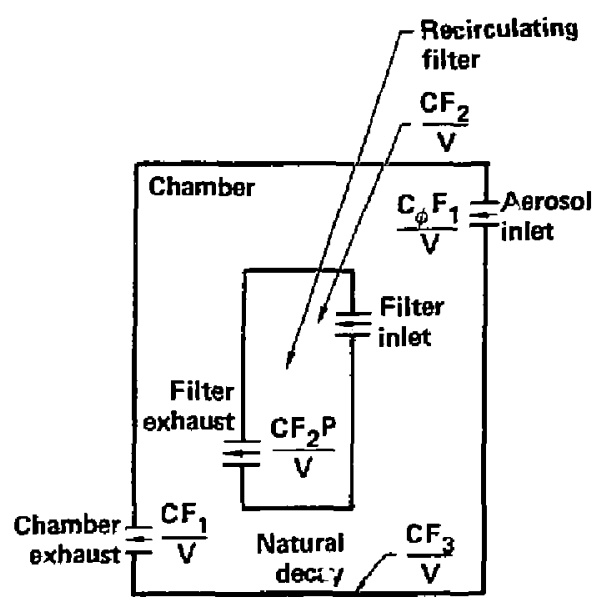

Fig. 113 Aerosol sources and sinks present with a recirculating filter in a conf ined chamber. 
the valumetric flow rate through the filter. And, finally, the particle concentration penetrating the filter supply adds another source, $\mathrm{CF}_{2} \mathrm{p} / \mathrm{V}$ where $P$ is the total filter penetration. The overall change in the chamber concentration is then given by the following differential equation:

$\frac{d C}{d t}=\frac{C_{0}}{V}\left(F_{1}\right)-\frac{C}{V}\left(F_{1}+F_{2}+F_{3}-F_{2} P\right)$

The final sink term takes into account al! natural losses within the chamber, such as particle tosses on the walls and gravitational setting. Since the filter efficiency, E, is 1 - P, the Eq. 82 can be rewritten as--

$\frac{d C}{d t}=\frac{C_{0}}{V}\left(F_{1}\right)-\frac{C}{V}\left(F_{1}+F_{2} E_{F}+F_{3}\right)$

to include only one source and three sink terms. Assuming that the chamber has been previausly equilibrated to an aerosol concentration of $C_{0}$, Eq. 83 may be integrated to yield

$C_{1}=C_{0}\left[\frac{F_{1}}{A}+\left(1-\frac{F_{1}}{A}\right) e^{-\frac{A_{T}}{v}}\right]$

where $A=F_{1}+F_{2} E+F_{3}$.

To see the effect of the recirculating air filter on chamber air cieaning, we are interested in the final equilibrium chamber concentration, which is reached after infinite time. For this case, Eq. 84 reduces to

$C_{\infty}=C_{0}\left[\frac{F_{1}}{F_{1}+F_{2} E_{F}+F_{3}}\right]$

Furthermore, if we assume negligible natural losses, $F_{3}=0$, then

$$
C_{\infty} \approx C_{0}\left[\frac{F_{1}}{F_{1}+F_{2} E_{F}}\right]
$$


So, the final equiliorium concentration is not simply a function of the filter efficiency, but also of the chamber and filter flow rates. If we let the relative filter flow rate be

$R=\frac{F_{2}}{F_{1}}$

Then, the relative concentration of particies remaining is

$\frac{C_{\$}}{C_{0}}=\frac{1}{1+R E_{F}}$

In order to achieve the lowest concentration of aerosols within a chamber, Eq. 88 indicates that we must use an air filter with the highest efficiency and operate it at a maximum air flow rate. Unfortunately, these two variables are inversely related since a filter with a higier efficiency usually has a higher resistance that decreases the flow through the filter. Thus, once a given $f i l t e r$ design and filter media are selected, the values of filter efficiency, $E_{F}$, and the relative filter flow rate, $R$, are fixed. The efficiency for cleaning the room, $E_{R}$, is defined as $1-C_{\infty} / C_{0}$ and $c$ an be computed using Eq. 88:

$E_{R}=\frac{R E_{F}}{1+R E_{F}}$

Equation 89 was used to calculate the room-clean.ng efficiency as a function of filter efficiency for filter flow rate ratios from 0.5 to 3.0 (Fig. 114). Several generalizations can be made based on Eq. 88 and Fig. 114. At low relative flow rates $(R<1)$, the room-cleaning efficiency wilt always be less than the filter efficiency since only a small fraction of the contaminated air is filtered. Equation 88 can be approximated by

$E_{R}=R E_{F}$

at very law relative flow rates. Figure 114 shows that for $R=0.5$ and low filter efficiencies, the room cleaning efficiency increases with increasing filter efficiency. 


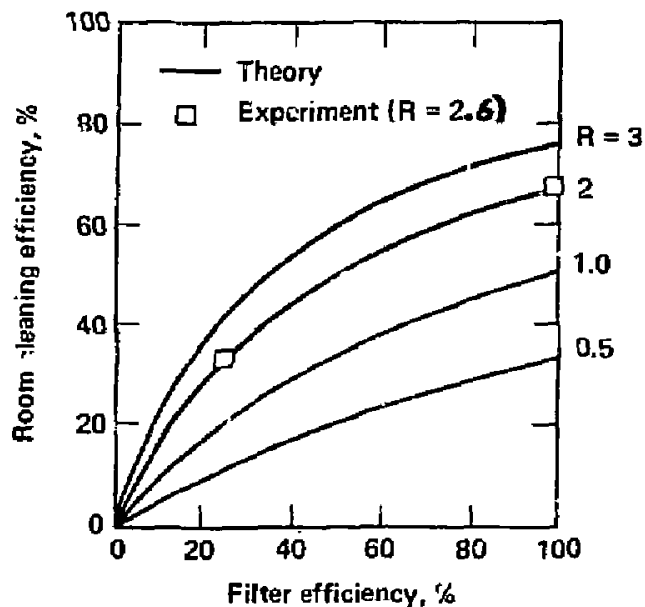

Fig. 114 Expected cleaning efficjency as a function of the recirculating Filtr:r efficiency for the flow rate ratios of $0.5,1.0,2.0$, and 3.0 . 
At high relative flow rates $\langle R>1\rangle$, the room-cleaning efficiency can he greater thall, equal to, or less than the filter efficiency, depending on the value of the fifter efficiency. If the filter efficiency is iess than a critical value, the room-cleaning efficiency will be greater than the filter efficiency. This occurs because the same air is filtered more than once. The fraction of the air filtered is greater than the fraction of particles removed by the filter. Under these conditions, Eq. B9 aiso reduces to Eq. 90. Figure 114 shows that for $R=2$ and 3 and iow filter efficiencies, the roomcleaning efficiency increases approximately linearly with filter efficiency in agreement with Eq. 90.

If the filter efficiency is greater than a critical value, then the roomcleaning efficiency will be less than the filter efficiency. This occurs when the fraction of air filtered is less than the filter efficiency. For a recirculating air filter, the fraction of air passing through the filter will always be less than $100 \%$ even for large values of relative filter flow rates. Since the filtered exhaust is continuously added to the air being filtered, a fraction of the air never passes through the filter. If the filter efficiency is greater than the fraction of room air being filtered, the room-air-cieaning efficiency will be less than the filter efficiency. For very high filter efficiencies, Eq. 89 reduces to

$$
E_{R}=\frac{R}{1+R}
$$

Equation 91 predicts that at high filter efficiencies, the room-cleaning efficiency is independent of filter efficiency and depends only on the relative filter flow rate. This behavior is seen in Fig. 114.

The critical filter efficiency, $\varepsilon_{C}$, at which the room-cleaning efficiency equals the filter efficiency is obtained from Eq. 89.

$E_{c}=1-\frac{1}{R}$

Equation 92 shows that the critical efficiency increases to higher values at higher relative fitter flow rates. Recirculating air filters having filter efficiencies lower than this critical value will have a room-cleaning efficiency that is higher than the filter efficiency. Conversely, if the filter efficiency is greater than the critical value, the room-cleaning 
efficiency will be less than the filter efficiency. The critical filter efficiency defines the boundary in room-cleaning efficiency between filter control and ventilation control. Thus, for low filter efficiencies, the roomcleaning efficiency is controlied by the filter efficiency as app-oximated by Er. 90, and for high filter efficiencies is controlled by the relative filter flow rate as approximated by Eq. 91 .

The previous discussion of roon-cleaning efficiency dealt with equilibrium canditions in which the aerosol generator's ventilation system and the recirculating air filter have been operating for a long time. However, as shown by Eq. 84, the concentration of aerosols in the chamber is a function of time. The time required to reach half the equilibrium concentration, $C_{\infty}$, can be derived from Eq. 24 to yield

$$
t_{1 / 2}=\left[\frac{\mathrm{V}}{\mathrm{F}_{1}+\mathrm{F}_{2} \varepsilon_{\mathrm{F}}+\mathrm{F}_{3}}\right] \ln (2)
$$

Equations 84 and 93 apply to the case where the racirculatirig air filter was turned on, with the chamber having an equilibrium aerojol concentration of $c_{0}$. The time was measured from the point the recirculating air filter was turned on and represents a basic constant for the total air-cleaning system. Note that the term, $V /\left(F_{1}+F_{2} E_{F}+F_{3}\right)$, represents the time for a complete air change in the cell.

Another important case occurs when the aerosol source is stopped or removed. The concentration of aerosols as a function of $t$ ime is ohtained from Eq. $B 3$ with the source terms $C_{0} F_{1} / V$ removed and the resulting differential equation intergrated to yield

$C_{t}=C_{0} e^{-\frac{A}{*} t}$

In this case, the final equilibriur, particle concentration is zero. The time required for the concentration to reach one half of this equilibrium concentration is also given by $\mathrm{Eq} .93$.

As we can see, the evaluation of a recirculating air filter involves not oniy the efficiency of the filter unit itself, but also the characteristics of the chamber such as size, existing ventilation system, and rate of aerosol production and dispersion within the chamber. For a given recirculation rate, 
the larger the chamber size and the greater the aerosol production, the lower the effectiveness of the recirculating air filter in reducing the chamber aerosol concentration. How well the aerosols disperse in the chamber is another important factor controlling the effectiveness of the recirculating air filter. If the aerosols do not disperse well within the chamber due to dead eaces or to air-flow patterns, the performance of the recirculating air filter will decrease accordingly.

\section{INITIAL DESIGN AND LABORATORY EVALUATION}

The initial design of our recirculating electric air filter minimized filter maintenance due to fi\}ter-clogging by particle deposits. Since many of the operations in the fuel-fabrication cell are performed with mechanical manipulators, even minor tasks requires a considerable effort. Even though lead-lined gloves in the ports on the rear wall of the cell exist for manual operations, the decreased dexterity and the increased radiation exposure negated any filter design requiring frequent use of the gloved ports. We designed the experimental filter to operate for long periods without maintenance, the basic design approach being the filter had a maximum dust-holding capacity. The remaining design criteria were maximum air flow, maximum filter efficiency, maximum dust recovery, minimum size, simple installation and removal, insensitivity to rough handing, minimum cost, and no high-voltage nazard. These criteria had to be optimized while meeting the primary constraint of the cell, that of the 14-inch diameter entry/exit port.

We optimized the maximum air flow and filter efficiency by selecting a blower that fits through the 14-inch port and pulls the maximum air flow against the resistance of a medium efficiency filter. These two criteria are inversely related since increasing the filter efficiency decreases the blower air flow due to the increasing filter pressure drop. The blower-filter combination that we selected pulls $380 \mathrm{cfm}$ and has $96 \%$ efficiency with electrification for submicron aerosols. The blower is a high-pressure centrifugal blower (IGL model 7-l/2 P) and the filter is a $1 / 2$-inch thick glass fiber mat (Johns-Manville, AF-18). This combination increased the total ventilation rate in the Savannah River plant facility from $80 \mathrm{cfm}$ to $260 \mathrm{cfm}$. According to Eq. 89 , this experimentai filter should reduce the particulate level in the cell by $68 \%$. 
Maximum dust recovery required a large amount of filter media thus, increasing the size of the filter unit. Moreover, in arder to recover the dust deposits, the filter surface that collects the particles had to be smooth, thereby eliminating the option of convoluting the media to increase its area while maintaining an overall small size. We roughly optimized the dust-holding capacity, small size and dust recovery with a cylindrical filter cartridge 1 foot diameter and 3 feet long. By mounting the electric motor, high-voltage power supply and other electrical components inside the filter cartridge, we minimized the size of the filter as well as the maintenance and potential contamination of the electric motor and other electrical components.

Our experimental filter is shown in Fig. 115. It is a completely self-contained unit and requires a standard $110 \mathrm{~V}$ input for its operation. The cylindrical filter cartridge slips over the filter housing and blower with the aid of a handle mouriced on top of the filter cartridge. Two clamps, nct shown in Fig. 115, secure the filter cartridge to the blower housing and provide an electrical ground to the outer electrode. Figure 116 shows the filter cartridge removed thereby exposing a grour. ad cylindrical screen. It protects the electrical components from physical dastage and provides a guide for inserting the filter cartridge. High voltage is applied tc the interior screen of the filter cartridge by means of a piano wire fastened to the high voltage lead that extends from the top of the cylindrical screen. The high-valtage output poses no electrical hazard since this power suppiy cannot exceed $70 \mu \mathrm{A}$ at $10 \mathrm{kV}$. Figure 116 also shows an electric contral box mounted on the blower housing with two toggle switches to operate the filter unit; a power switch to activate the blower and a separate high voltage switch to provide high voltage to the filter cartridge.

The cast iron blower and electric motor serve as the base of the filter unit and prevent the unit from tipping over if it is bumped or roughly handled. We modified the blower configuration so that filtered air will be pulled past the electric motor into the blower housing and exhausted through the part. This modification significantly reduces the size of the filter, lowers the center of oravity for increased stability, and places the electric motor inside the filter cartridge, thereby preventing motor failure due to dust particles. Since all of the filtered air are pulled across the electric motor, there is adequate motor cooling. 


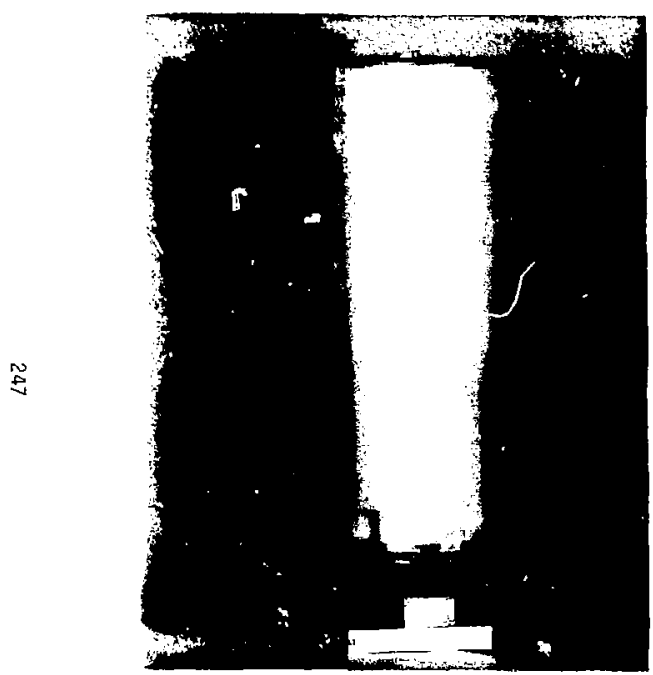

Fig. 115 Recirculating electric air filter.

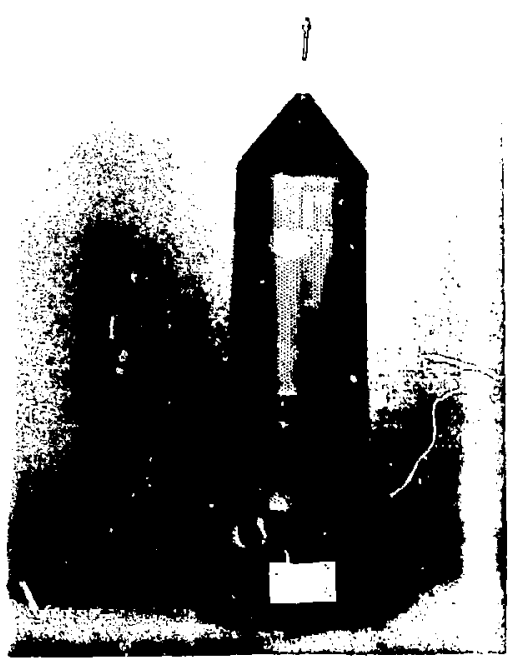

Fig. 116 Recirculating electric air filter with the filter cōrtridge removes. 
The rectangular box at the botton is the solid-state, high-voltage power supply. With a 15-volt DC input, it provides $10 \mathrm{kV}$ output to the $\mathrm{h}^{\text {i }} \mathrm{y}^{\text {h-voltage }}$ lead at the $t u$, if the unit. A string of three resistors in series connect from the high voltage lead on top to a logic circuit that senses if there is an overload. If there is an excessive drain on the power supply due to some failure mode, a light on the electrical control box turns on.

Labor.tory Evaluation. The electric recirculating air filter was evaluated in an LI.NL test cell under controlled laboratory conditions designed to simulate actual operation in a cell of the ${ }^{23 \mathrm{PuO}_{2}}$ fuel-fabrication facility in the Savannah River Plant. NaCl or dioctyl sebacate (D05) aerosols were injected into the cell tinough the ventilation inlet. Our evaluation consisted of measuring the efficiency of the filter unit and determining hiw we' 1 :ne ex,erimental filter reduced the particle concentration in the cell. The evaluation was conducted by measuring the aerosol concentration in the blower exhaust and the aerosol concentration in the cell as a function of time after the recirculating air filter was turned on. These measurements were made using a light-scattering photometer and $\mathrm{NaCl}$ aerosols having a AMMD $=0.8 \mu \mathrm{m}$ and $\mathrm{a} x \mathrm{~g}=2.0$. The pressure drop across the fitter media was $0.2 \mathrm{in}$. water with the blower operating at $180 \mathrm{cfm}$.

The aerosol concentration decay curves are shown in Fig. 117 for the two cases corresponding to operation with no high voltage and with $10 \mathrm{kV}$ applied. For each case, the concentration in the room and the concentration exiting the recirculating filter was $23 \%$ with no high voltage and $96 \%$ with the applied voltage. The time required to rucice the particle concentration in the room to half of the final reduction achieved at equilibrium was 2.9 minutes with $0 \mathrm{kV}$ and 1.4 minutes with $10 \mathrm{kV}$ applied to the filter.

Table 17 shows that the theory overestimates the room cleaning efficiency and underestimates the time required for the particle half life. The primary cause for the discrepancy between theory and experiment is the non-ideal mixing of the aerosols in the chamber volume. We had assumed in deriving the rheoretical equations that the particles would be instantly and uniformly mixed throughout the chamber volume. This assumption is not correct since a finite amount of time is required for the particles to be uniformly mixed in the chamber. The lir flow entering the recirculating air filter $\left(F_{2}\right)$ and the air flow exiting the chamber $\left(F_{1}\right)$ would not have a uniform part.cle 


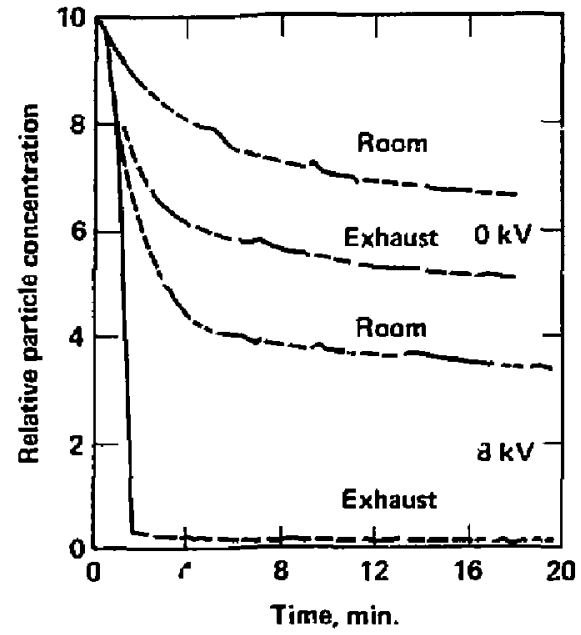

Fig. 117 Decay of particle concentration with and withot an applied electric field in a confined chamber using a recirculating air filter.

Table 17. Comparison of theoretical and experimental data for recirculating air filter (design 1).

\begin{tabular}{|c|c|c|c|c|c|}
\hline \multirow[b]{2}{*}{ Voltage } & \multirow{2}{*}{$\begin{array}{l}E_{F} \\
(q) \\
\text { Exp }\end{array}$} & \multicolumn{2}{|c|}{$\begin{array}{l}\mathrm{E}_{\mathrm{B}} \\
(8)\end{array}$} & \multicolumn{2}{|c|}{$\begin{array}{l}t ! / 2 \\
(m i n)\end{array}$} \\
\hline & & Exp & Theory & Exp & Theory \\
\hline $0 \mathrm{kV}$ & 23 & 33 & 37 & 2.9 & 2.2 \\
\hline $10 \mathrm{kV}$ & 96 & 66 & 71 & 1.4 & 1.0 \\
\hline
\end{tabular}


concentration. This results in a reduced effective air flow. Since the particle half life is inversely dependent on the total air tlow which removes paiticles from the chamber, it can be used as a measure of the effective air flow. Comparing the theoretical and experimental half lives in Table 17 shows that the eifective air flows are reduced by sbout $30 \%$.

The experimental fiiter efficiency and room-cleaning efficiency from Table 17 was plotted in Fig. 114. The lower and higher efficiency points represent the performance of the recirculating air filter at $0 \mathrm{kV}$ and $10 \mathrm{kV}$, respectively. Although the experimental relative filter flow rate, $R$, was 2.6 , the data fall on the curve for $R=2.0$. This shows that the effective fjlter flow rate is reduced by $30 \%$. Figure 114 also shows that the lower efficiency filter produces a higher room cleaning efficiency while the $h$.yner efficiency filter produces a lower room cleaning efficiency when compared to the filter efficiency values.

After completing our laboratory evaluation of the recirculating air filter, we shipped the filter to the Savannah River Plant for installation in the Pu fuel fabrication cel1. During the on-site review of the experimental filter, we discovered that the recirculating air filter did not meet all of the stringent requirements for installation in the cell. The primary concern with the experimental filter was the fear of contaminating the highly pure $\mathrm{PuO}_{2}$ in the cell with $\mathrm{SiO}_{2}$ from the filter fibers that might break of from the electric filter. Because even a small amount of contamination by almost any element would ruin the $\mathrm{PuO}_{2}$ product, the experimental filtir could not be installed in the fuel fabrication celi. Although HEPA filters made from glass fibers are also used in the cell, they do not present a contamination problem since the filtered exhaust is directed away from the cell. Another possible chemical contamination from the painted surfaces of the electric motor and blower flaking off was avoided by removing all the paint.

Another concern with the design was the residuai contamination on the filter cartridge when it would be tecomissioned. Based on our previous experience with the dust-holding capacity and the anount of residual dust remaining after cleaning, we estimated the recirculating air filter would have a maximum dust loading of 235 grams after 9 months of operation and a residual deposit of 47 grams after cleaning. Since any item containing more than 10

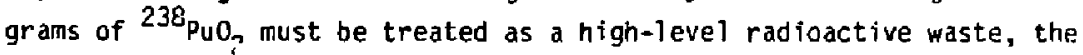
experimental fil ter would clearly be classifed as high level waste. Th? 
management of the PI fucl-iabrication facility has a strict policy of minimizing all high-level waste, therefore, the operating procedure for the recirculating electric fiter would have to be changed. Instead of allowing the particles to buid up a deposit on the filter over many months and periodically recovering the material by cleaning, the experimental filter would have to be replaced every two to four weeks to ensure hat the filter contained no more than 10 grams of $\mathrm{PUO}_{2}$. This restriction would eliminate any material recovery and add substantially to the operating cost of the filter. HEPA filters in the cells are also replaced every month to keep $\mathrm{PuO}_{2}$ deposits under 10 grams.

\section{REDESIGNED RECIRCULATING AIR FILTER}

In order to meet the requirements of preyenting chemical contamination arid keep the residual deposit of the $\mathrm{PuO}_{2}$ particles below $10 \mathrm{grams}$, we had to completely redesign the filter cartridge. The original filter cartringe consisted of high voltage and ground electrodes, filter media and support and sealing $f$ langes. This unit was designed to have repeated cycles of particle qeposit rormation and removal before the entire anit was discarded. Replacing the glass fiber media with chemically acceptable media or imobilizing the glass fiber media by using porous metal alectrodes met the chemical contamination criterion, but did not meet the 10-gram limit if the filter were aperated to the maximum dust-holding capacity.

Replacing the filter cartridge every two to four weeks was an impractical solution because of the excessive purchase cust and labor cost for continually bagging new and used filter cartridges in a'id out of the cell. Moreover, since the filter artridge design had a rigid high-voltage electrode made from perforated aluminum and reinforcement and sealing fianges, the unit could not be easily disassembled or compressed for reduction of the volume of waste.

We concluded that the overatl design of a cylindrical filter cartridge mounted on a blower was still good, but instead of the filter cartridge being an incegral unit, the cartridge was designed for easy disassembly and replacement of the filter media using a hinged outer electrode. The outer grolind electrode is removed by releasing three compression clamps and opening the hinged electrode. Figure 118 shows the filter cartridge with the outer ground electrode partially removed. 


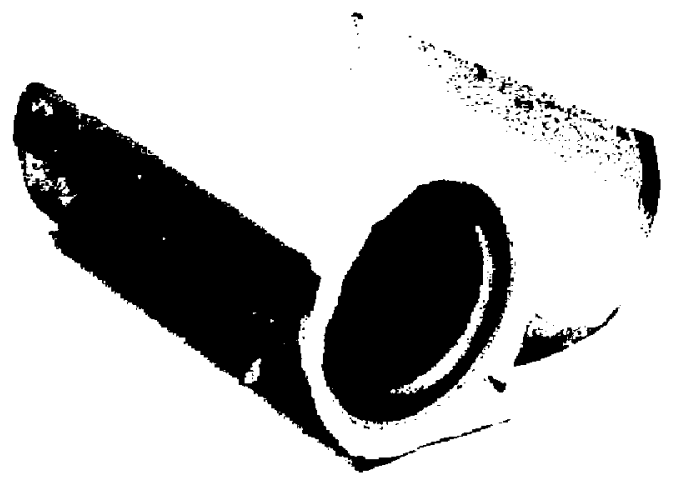

Fis. 118 Filter cartridge with outer ground electrode partially removed. 
Once the outer ciectrode is removed, the filter media can be easily replaced by unwrapping a flat sheet of filter media from around the central electrode. The central electrode is a cylinder of perforated aluminum and is reinforced at both ends and in the middle. A plexiclas flance provides a sealing edge for the filter cartridge on the blower nousing. The other end is sealed with a flat Plexiglas plate to ensure that ail of the air fluws through the filter media.

The improved filter design was made possibis because a varie:y a: nolymeric filter media, made from various hydrocarbons such as polyolefin. art polyesters, can be used in the fuel-fabrication cell which do not contaminate the $\mathrm{PuO}_{2}$ powder. If hydrocarbon fibers from a filter become mixed with the $\mathrm{PuO}_{2}$ powder, they will be rapidly oxidized to carbon dioxide and water when the $\mathrm{PuO}_{2}$ powder is heated in a high temperature oven.

We tested vartous polyolefin and polyester filter media individualiy and in combinations in our small-scale filter test facility. Each test consisted of measuring the filter efficiency with and without high voltage at a filterface velocity of $15 \mathrm{~cm} / \mathrm{s}$ which corresponds to the face velocity in the redesigned recirculating air filter. Many of these filters showed larqe increases in ilter efficiency as high voltage was applied. For example, the efficiency of a polyester filter was $13 \%$ at $0 \mathrm{kV}$ and $70 \%$ at $10 \mathrm{kV}$. The pressure drop across this filter was only 0.07 inch of water. Unfortunately, these filters, were very difficult to install. When the outer electrode was being $c$ lamped around the filter media, the media would distort severely and form wrinkles and convolutions. So, we were not able to use these polymeric filters.

We finally selected a needle-punched polypropylene (Verto company, The Netherlands). This media is made from electret fibers and was discussed in detail in the sectior. on permanently charged filters. Since we wanted to use the Filtrete media to demonstrate the performance of externally charged media and not permanently charged media, we sprayed the media with an anti-static agent to remove the charge.

The G-1200 Filtrete media was easily installed in the filter cartridge. It was very pliable and remained in place when wrapped around the central electrode. The media was sufficiently thin (3/8 inch) to avoid ristortions as the oxter e?ectrode was being clanped together. It also had sufficient 
compressibility to ensure an adequate seal around the top and bottom flanges when the outer electrode was clamped in place.

The use of an easily replaceable filter media like the Filtrete G-1200 eliminated the problem of cherical contamination and also allowed the filter to be used as intended. The filter operated without maintenance as particle deposits built up on the filter. When these deposits reached a point restricted the air flow, the particle deposits were recovered by shaking or knocking the filter and the cycle repeated until the filter media was ready for disposal. To meet the restriction of having less than 10 grams on each decamissioned item, the filter media would be easily cut into a number of smaller segments. This approach al lowed for the recovery of $\mathrm{PuO}_{2}$ dust while complying with the io-gram limit on disposable items.

\section{Laboratory Evaluation}

The laboratory evaluation consisted of measuring the concentration of $\mathrm{NaCl}$ particles in the chamber and in the filter exhaust as a function of time after turning on the recirculating air filter. Separate tests were conducted with $0 \mathrm{kV}$ and $8 \mathrm{kV}$ applied to the central electrode to yield similar results to those shown in Fig. 117. The filter pressure drop in both tests was 0.11 inches. The test with $8 \mathrm{kV}$ showed the filter had an efficiency of $84 \%$ and produced a room cleaning efficiency of $65 \%$. The filter efficiency with no applied voltage was $75 \%$ and produced a room cleaning efficiency of $62 \%$.

The unusually high filter efficiency with no applied voltage was due to the residual charge on the filtrete media that could not be neutralized. At the low filter-face velocities used in these tests, even a small residual charge would produce a high filter efficiency. However, at higher flow rates, the filter appears to be discharged. For example, at $65 \mathrm{~cm} / \mathrm{s}$ the same media only has an efficiency of 10\%. Despite the ambiguity in the contribution of the external charging and the permanent charge on the total filter efficiency, the superior handling properties of the filtrete media made it the best choice for our field evaluation of the recirculating electric air filter. 
Field Evaluation of Improved Design Recirculating Electric Air Filter at Savannah River

We installed the recirculating electric air filter in the ${ }^{238} \mathrm{Pu}$ fue fabrication facility in - Ilding 235-F at the Savannah River Plant, South Carolina. This facilicy consists of nine interconnected cells in which ${ }^{238} \mathrm{PuO}_{2}$ powder is forned into fuel pellets that serve as a heat source for thermo-electric generators. The process of converting powder into finished pellets is primarily done using remote mechanical manipulators; a number of operations are also performed using lead-lined gloves. There are two electric motor hoists in Cell 1 and one in Cell 5 for transporting material.

The entire operation in the nine cells can be grouped into th e major parts according to the form of $\mathrm{PuO}_{2}$ and the leval of suspended particulates. In Cells 1 and $2, \mathrm{PuO}_{2}$ is a fine powder and the cells have a high concentration of suspended particulates. The particles become suspenc $f$ in the pouring and other powder-handling operations. Cells 3-5 handle the $\mathrm{PuO}_{2}$ in the form of large granules or a monolithic pellet and result in a relatively low concentration of suspended particles. Cells 6-9 have a negligible concentration of suspended particles since the $\mathrm{PuO}_{2}$ is sealed inside welded containers.

The operational problems created by suspended particles can likewise be categorized according to the three parts with cells 1 and 2 reprcsenting the worst case, Cells 3-5 an intermediate case, and Cells 6-9 having no operational problems created by suspended particles. Among the operational problems created by the radioactive dust particles are premature failure of the mechanical manipulators, electric hoists, gas lines, thermocouple leads, lead-lined gloves, and vacuum gauges. The failure in each of these cases is due to the intense radioactivity directly destroying the component or weakening the component's strength sufficiently to cause a premature mechanical failure. In addition to these direct problems, the suspended particles also require a frequent change-out of HEPA filters.

Since the powder-handling operations in Cell 1 produced the highest. concentration of suspended particles and the greatest maintenance problems, we insialled our recirculating electric air filter in this cell. Figure 119 shows the filter unit positioned next to a high-temperature oven that is mounted in the floor. Glove ports are used for pouring $\mathrm{PuO}_{2}$ powder into the oven and removing the sintered product. Prior to the installation of the 


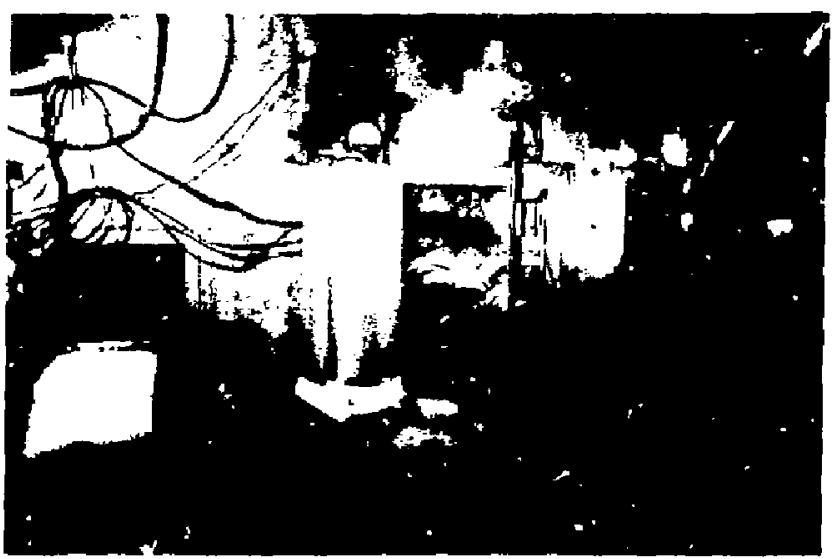

Fig. 119 Recirculating electric air filter installed in Savannah River's Cell I next to a high-temperature furnace. 
electric filter, a sample of the filter media, shawn in the left side of Fig. 119, was placed in the cell to determine any deterioration in the harsh radioactive environment. Although the filter sample visually appeared to be fine, it had, in fact, degraded significantly during the four weeks it was placed in the cell. Unfortunately, when we placed the recirculating electric air filter in the cell, we did not physically exarine the sample media for degradation. The sample media was very sticky, indicating that the fibers had melter.

The evaluation of the recirculating electric air filter consisted of measuring the concentration of suspended $\mathrm{PuO}_{2}$ particles bef re and after the fitter as a function of time. We used a light-scattering protometer commercially available from Air Techniques Inc. in these measurement. Since penetrationr in the chamber wall were not permitted, we placed the article measuring system inside the celt.

The results of our evaluation are shown in Fig. 120 where we have graphed the relative photoneter measurements in the chamber and in the filter exhaust as a function of time. We encountered a considerable amount of baseline drift in the photometer and frequently adjusted th: electronic meter. After 80 minutes into the evaluation, the baseline shifi. could no longer be adjusted electronically and had to be subtracted from the phutometer measurements. The photometer measurements in Fig. 120 show a sharp increase in the chamber dust concentration when a variety of powder-handling operations are commenced at 8 minutes, and a gradual decay in the concentration when the operations are teminated at 50 minutes. The concentration of $\mathrm{PuO}_{2}$ dust in the chamber then steadily decreased since the remaining operations in the cell did not involve handling exposed powder. All of the operations in Cell 1 , except for the filter eulluation, were terminated after 120 minutes.

Figure 120 sho's that the electric filter was operated for 66 minutes with no applied voltage and for 44 minutes with $8 \mathrm{kV}$. During these periods, the photoneter mecsurements were alternately taken from the chamber and the filter exhaust, thereby allowing filter-efficiency calculations. Figure 120 shows that the recirculating air filter had an average efficiency of $23 \%$ with no voltage and $50 \%$ with $8 \mathrm{kV}$. The higher filter efficiency for the $0 \mathrm{kV}$ filter that occurred during the sharp increase in the chamber concentration was probably due to a larger particle size. 


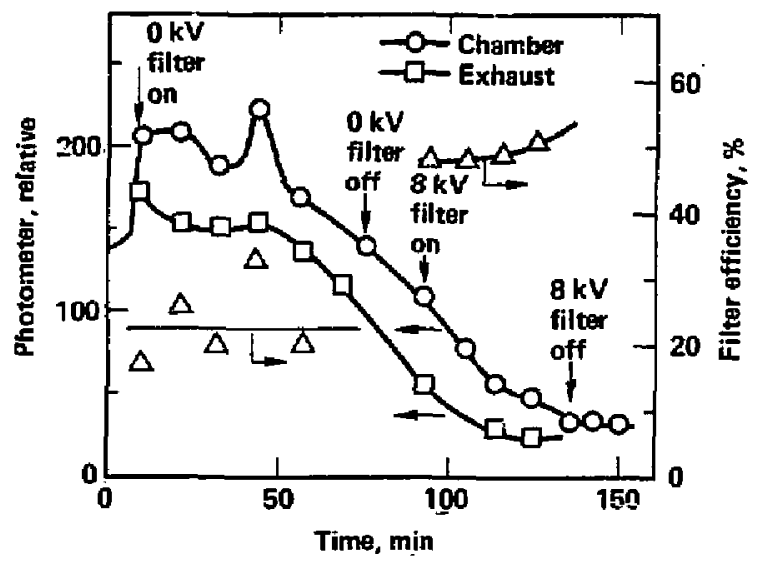

Fig. 120 Results of field evaluation of recirculating electric air filter in 23 Q $\mathrm{uO}_{2}$ cell at Savannail River Plant. 
Since Cell 1 had open passagas to a wing cabinet and to Cell 2 , the volume, $V$, used in the half-life calculations was the total of all of these chambers, that is $920 \mathrm{ft}^{3}$. The existing ventilation $\mathrm{flow}, F_{1}$, consisted of a HEPA filter in cells 1 and 2, yielding a total fjow of $160 \mathrm{cfm}$. The final data needed for the half-life calculations were the flow rate $(180 \mathrm{cfm})$ and the efficiency $(23 \%$ at $0 \mathrm{kV}$ and $50 \%$ at $8 \mathrm{kV}$ ) of the recirculating electric air filter. We obtained a half-life of 3.2 minutes for the $0 \mathrm{kV}$ filter and 2.6 minutes for the $8 \mathrm{kV}$ filter.

The particle concentration data in Fig. 134 steadily decreased after 50 minutes with a haif-life of about 40 minutes, This half-? ife was $1 \%$ to 16 times onger than the calculated half-life.

We believe that the primary cruse of the large difference between observed and calculated half-life is an ext-emely slow dispersion of pu dust throughout the cell volume. The cold pressing of the pu powder is done in a wing cabinet that projects 4 feet out of cell 1. Dust generated in this cabinet mixes very slowly with the air in the main portion of Cell 1 since it has no ventilation flow to disperse the particles. In addition, the 14-inch diameter door that separates Cells 1 and 2 presents a major obstacle to the uniform mixing of particles in both cells. Because of these restrictions to the uniform dispersal of Pu dust throughout the cells, the effective ventilation flow will be much less than the actual flow. Even for the ideal flow system we used in our laboratory studies, we found that the effective flow rate was only $70 \%$ of the actual flow rate. Considering the complex air flow in the two cells and the wing cabinet and all of the "dead" spaces, it is reasonable to expect that the effective air flow is about $10 \%$ of the actual flow rate. An effective flow rate that is $6 \%$ of the actual flow rate will yield the observed 40 minute half-life.

The most important information from our field evaluation wass the effect of the recirculating electric air filter on the total reduction of particles in the chamber. He can estimate the room-cleaning efficiency using Eq. 89 and $R=1.13$ and $E_{F}=0.23$ and 0.50 for $0 \mathrm{kV}$ and $8 \mathrm{kV}$, respectively. These calculations show that the recirculating air filter with $0 \mathrm{kV}$ will reduce the particle concentration in Cells 1 and 2 by $22 \%$ and by $36 \%$ with $8 \mathrm{kV}$. Comparing these results to the curves in Fig. 114 shows that the low relative filter flow rate, $R$, and the low filter efticiency, $E_{F}$, combine to produce low room-cleaning efficiencies. 
When we designed the experimbi.al riter, we did not realize that the door between Cells 1 and 2 is always kept spen thereby requiring both cells to be cleaned. If the interconnecting door was closed, then the recirculating air filter would only have to clean Cell 1. The resulting room-cleaning efficiency would be $36 \%$ with $0 \mathrm{kV}$ and $53 \%$ with $8 \mathrm{kV}$. The unexpected low filter efficiency also contributed to the relatively low room-cleaning efficiency. Our laboratory studies with $\mathrm{NaCl}$ aerosols showed an efficiency of $85 \%$ which should have produced a room-cleaning efficiency of $49 x$ for both cells 1 ar.d 2 .

In addition to a low efficiency, the recirculating electric filter also experienced an unusually high pressure drop. The pressure drop across the electric prefilter was 0.6 inches throughout the evaluation. This was three times higher than the 0.2 inches obtained in our laboritory tests. Although an increase in the filter pressure drop is usually accompanied by an increase in the filter efficierioy, we had obtained the opposite results. We suspect that the filter media had somehow deteriorated in the harsh radioactive environment since the exposed portion of the media at the base of the filter was extremely frayed. The filter media was remo ifor inspection. In handling the filter media with gloves and mechanical manipulators, we observed that the media behaved very much like cotton candy, sticking to itself and whatever was in contact with it.

The source of the filter deterioration was ${ }^{238} \mathrm{PuO}_{2}$ particles that behaved as microscopic heat sources to nelt the surface of the fibers. ihis occured because the high specific antivity of the Pu- ${ }^{238}$ generates a considerable ariount of heat. Although the individual Pu particlas cannot melt the polypropylene filter mat, they can melt the surface of individual fibers. Since heat is continuously generated, the surface of the fibers will be liquid and make the filter mat appear to be sticky. Since the polypropylene filter was under compression between the two electrodes, melting the fiber surfaces caused the fibers to stick togither and thereby increased the resistance to the air flow. The recirculat. ag electric filter was placed inside its cell for almost one month before the filter was evaluated. During this time a sufficient amount of $\mathrm{Pu}$ dust particles coated the filter, even without the filter operating.

Once we discovered that the recirculating electric filter now had to have high-temperature resistance, we concluded that our filter design could not meet all of the constraints. The recirculating electric air :ilter did meet 
the other requirements of preventing chemical contamination, recovery of $\mathrm{Pu}$ dust, and a maximum Pu rontamination of $10 \mathrm{grams}$ per item at disposal. If a high-temperature filter mat, such as a fibrous alumina filter were used in place of the polypropylene filter, then the filter design could also meet the high-temperature requirements as well as the $1 \mathrm{C}$. ngram limit on residual Pll $_{\text {and }}$ recovery of $P_{u}$ dust. The filter design, however, cannot simultaneously meet al1 of the requjrements as it is currently designed.

HIGH-TEMPERATURE ELECTRIC AIR FILTER USING A STACKEO-0ISK DESIGN

DESIGN

As a result of our experience with the recirculating air filte- at Savannah River, we developed a high-temperature electric air filter that would prevent chemical contamination, - $t$ allow for recovery of collected dust, and have less than 10 grams of residual deposit when the filter is discarded. Our new filter design also occupied far less space, thereby minimizing any interference with cell operations.

We were motivated in this design by the conclusiuns of our cosi/benefit analysis and our field evaluation of the giove-box prefilter at the Rocky flats Plant. We had learned that the most cost-effective filtration system had a very high efficiency and could be cleaned many times prior to its disposal. A critical finding was that the dust-holding capacity did not have to be very high, provided the filter could be repeatedly cleaned. Moreover, since highefficiency filter media also have a high pressure drop, the filter design had to accimodate a large filtering surface. To accomodate a large filter surface within : relatively small volume, the filter media must be packaged in a configuration that placed filtering surfaces in close proximity to each other. Unfortunately, packaging a large filter surface in a relatively small volume does not illow recovery of deposited material. For example, the pleated configura:ion of the filter media in HEPA filters makes the filtering surface inaccessible for filter cleaning.

The successful design of a high temperature electric filter was therefore dependent upon finding a satisfactory configuration that would create the maximum filtering surface and yet be readily cleaned. HEPA filters having a 
pleated configuration could not be cleaned because the pleats could not be separated for cleaning, but allowing the adjacent filter sheets to be separated during the cleaning cycle was the key to a successfut design. We stacked individual disk filters together in a common exhaust port. Figure 121 shows the basic concept of this design which shows five filter disks stacked together to form a compact, high-efficiency filter. Each side of the filter disk is made from an appropriate filter medium. Contaminated air is filtered by the filter media and passes through the hollow interior and into the central opening. When the filter is clogged, the individual disks can be removed and the deposits brushed or shaken off. After cleaning the individual disk filters, they can be restacked and the filter unit placed back into service.

To meet the high-temperature requirements of the Savannah Rivel application, all of the materials used in the disk needed high-temperatura resistance. The selection of the outer filter surface was critical to a successful design. It. had to be smooth to allow deposits to be readily removed by brushing or other mechanica, means. It also had to have a high efficiency so that particles buil up on the surface rather than penetrating deep inside the media. The surface filter had to be structurally strong to withstand repeated cleanings without breaking off any of the filter media.

The candidate that met most of these requirements was a sintered fibrous stainless-steel filter available from the Bakaert Company, Belgium. This filter media has a significantly lower pressure drop than the more common stainless-steel filters made from sintered powder. The renuirement that the Bakert filter did not meet was efficiency. Laboratory test conducted at a face velccity of $23 \mathrm{~cm} / \mathrm{s}$ showed an efficiency of only $61 \%$ whiln tested with heterodisperse $\mathrm{NaCl}$ aerosols.

In order to obtain the necessary high efficiency, we converted the disk filter to an electric air filter. We added a filter made from alumina fibers (Saffil media) and a high-voltage electrode below the stainless-steel filter. Figure 122 shows a layer of the Saffil alumina filter being added next to the outer stainless-steel meos:!m. A portion of the Bakaert stainisss-steel filter is shown uncovered. The high voltage electrode is then placed over the Saffil filter, seen in Fig. 123. Insulation strips or polyethylene are inserted around the interior and exterior perimeter of the high voltage electrode to prevent short circuiting with the grounded exterior ring and the grounded interior exhaust flange. 


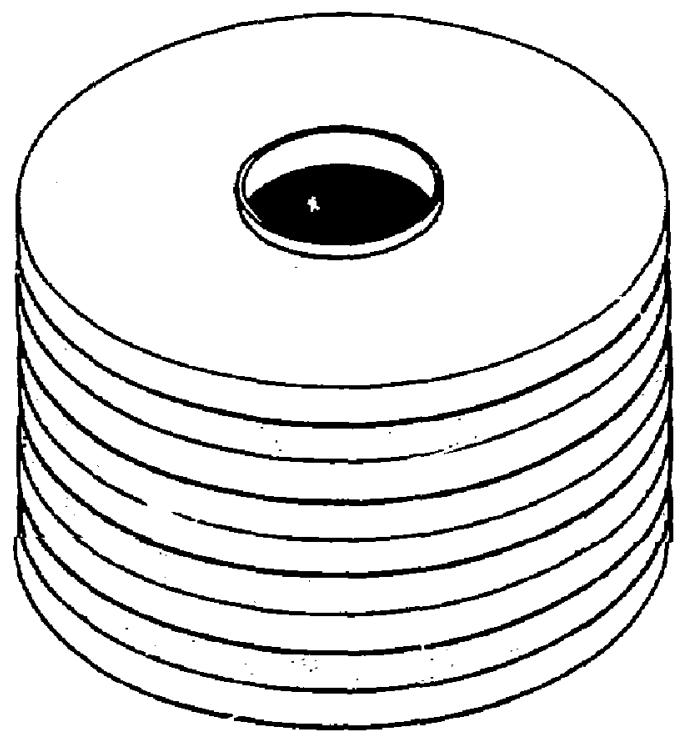

Fig. 121 High-temperature electric air filter consisting of five disks stacked together.

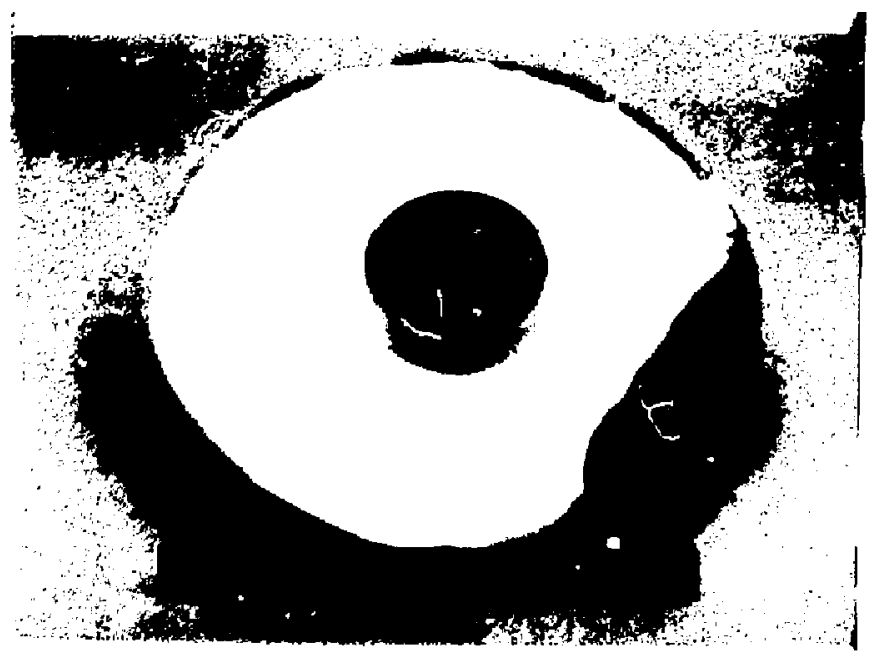

Fig. 122 Partial assembly of electric disk filter with fibrous alumina mat nearly covering the grounded outer electrode. 
The high voltage electrode provides the hollow space in the disk filcer through which the filtered exhaust flows. The filter ed exhaust exits the disk filter through slots in the central exhaust flange seen in Fig. 123. The remainder of the electric disk filter was assembled by adoing another layer of Saffit media over the high-yoltage electrode followed by the Bakaert stainlesssteel filter. The assembly was completed after welding the Bakaert media to the perineter ring. Figure. 124 shows the fully assembled high-temperature electric air filter. Note that the exterior Bakaert media completely encases the Saffil media, thereby preventing possible contamination of alumina fibers.

\section{Laboratory Evaluation}

We evaluated the performance of the high-temperature electric disk filter in our smal1-scale filter test laboratory. The filter penetration was measured as a function $u$, applied voltage using heterodisperse $\mathrm{NaCl}$ aerosots and a light-scattering photometer. Figure 125 shows the resuits. With no applied voltay: the filter had a penetration of $5 \%$. Increasing the applied voltage produced a logarithmic decrease in penetration unt if the penetration reached $0.02 x$ at $5 \mathrm{i} \cdot "$. The $h$ i $\mathrm{s}^{2}$-temperature electric filter has a penetration comparable to a HEPA filter. Further evaluation will be conducted on this electric air filter to better define its performance. One of the key factors that has yet to be established is how to clean these filters. Although these tests have not yet been conducted, we are confident of success based on our previous experience with cleanable filters.

\section{ROLLING ELECTRIC PREFILTER}

\section{INFRODUCTION}

A rolling electric prefilter (REP) was developed for use in ventilation systems in the nuclear industry to prevent the plugging of HEPA filters during a fire episode while also extending the lifetime of HEPA filters during routine use. Under nomal conditions, the REP behaves in a manner similar to the stationary electric alr filter; described previously. We electrified the rolling prefilter because it resulted in : higher collection efficiency 


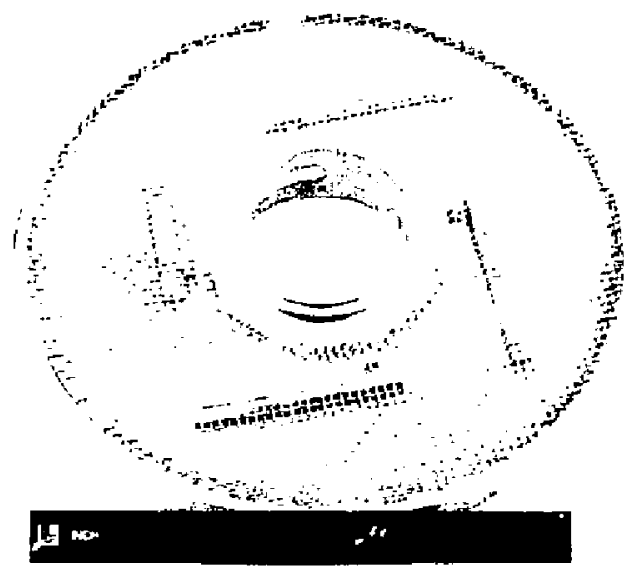

Fig. 123 Central high voltage electrode placed over the alumina mat and electrical insulation added.
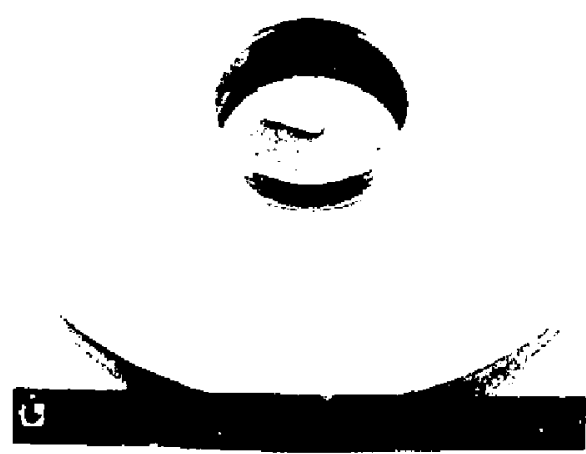

Fig. 124 High temperature eleciric air filter designed as a disk. 


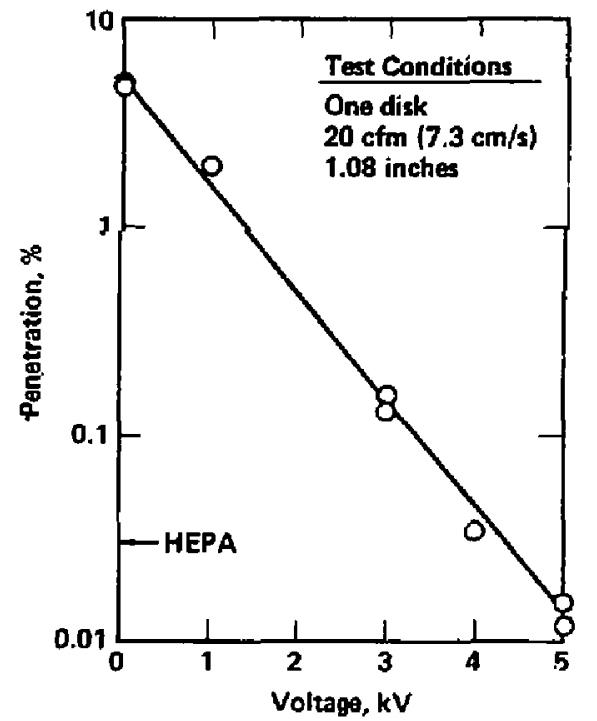

Fig. 125 Penetration of NaCl aerosols through an electric disk filter as a function of applied voltage. 
and longer filter life than conventional fibrous filters due to the added electrical capture mechanisms. The REF concept allows for the continuous replacement of filter media once it has plugged. The media can be advanced autcmatically subject to the pressure loss across the filter. Since the probali, ity of hauing a fire is very low, the multi-pass REP functions orimarily as a stationary prefilter. From an operational point of view, the REP would reduce maintenance costs associated with replacing stationary prefilcers significantly, but would require more space, and cost more than our stationary electric prefilter. The high initial cost of the REP wauld be offset by the significantly reduced maintenance costs for replacing stationary prefilters.

The application of the REP in a nuclear ventilation system has to be determined an a basis of a cost/benefit study and a risk analys is of fire hazards. For applications where the maintenance cost is very high, the use of the REP is justified in a cost/benefit study even if the risk is very low. In this case, the savings in maintenance costs would offset the capital cost of the REP. The use of the REP is justified in ventilation systems that have high fire risks even though the maintenance costs are very low.

DEFINITION OF PROBLEM

One of the most serious zrcidents that can occur in a nuclear facility is a fire. A critical factor that increases the severity of a fire is the potential loss of the ventilation system due to the plugging of HEPA filters by suloke aerosols. The following thrce countermeasures can mitigate the fire hazard:

1) materials managemert to reduce the quantity of combustible materials,

2) conventional fire detertion and fire suppression techniques, and

3) smoke removal or treatment in the ventilation duct upstream of the HEPA filter.

Among various methods examirs. for in-duct countermeasures were wet scrubbers, afterburners, granular beds, and prefilters. Although efficient prefilters appeared to stop the smoke aerasols, they were also plugged rapidly. Because of the tar-like property of the smoke aerosols, cleanable prefilters, like those used in bag houses, were not feasible. Once the smoke aerosols deposited on a filter, the particle deposit could not be removed. 
The severity of the filter plugging by smoke aerosols is illustrated in Fig. 126 which shows a section of filter media that has been exposed to smoke aerosols generated from combustion in a confined space. Tarry smoke deposits have solidified on the filter surface. Examination of these deposits under an lectron microscope show that the smoke deposits form a solid, impenetrable 1 ayer on the filter surface. This type of particle deposit differs significantly from the deposits seen previously in soiid NaCl particies. In contrast to smoke deposits that form a solid impenetrable barrier, the particle deposits seen in $\mathrm{NaCl}$ tests restrict the air passages but still leave open passages through which air can pass.

We should point out that the deposit of smoke aerosols shown in Fig. 126 resulted from snoke generated in an o.ygen-starved fire. The smoke aerosols in these fires are primarily liquid particles generated from the pyrolys is of the wood and plastic fuel. In contrast, smoke aerosols generated from we11ventilated fires are primarily solid hydrocarbon particles typicaliy characterized as soot. Because of these differences in the physical properties, the smoke aerosols produced in well-ventilated fires produce a gradual filter plugging while the smoke aerosols produced il: oxygen-starved fires produce an extremely rapid filter plugging.

\section{FEASIBILITY STUOY OF ROLLING ELECTRIC PREFILTER}

Any prefiltration technique for removing smoke aerosols would require some means for replacing the prefi;ter automatically once it had plugged. Two general approaches can be taken for replacing the plugged filter: replacing individual filter units in a batch process or replacing the filter medium in a continuous process. Since the filter industry had nothing available for raplacing individual filter units but has an extensive selection of rolling filters, we selected the rolling electric prefilter (REP) concept for a feasibility study.

Our objective was to establish the feasiblity of a REP that could maintain a low pressure drop and still protect the HEPA filter from plugging due to smoke aerosols. Since there were no commercial REPs that had the required high filter efficiency and low pressure drop, we modified filter housings and filter media of comercial units until we obtained the desired results. The modifications we made to the filter housing consisted of removing sharp edges 


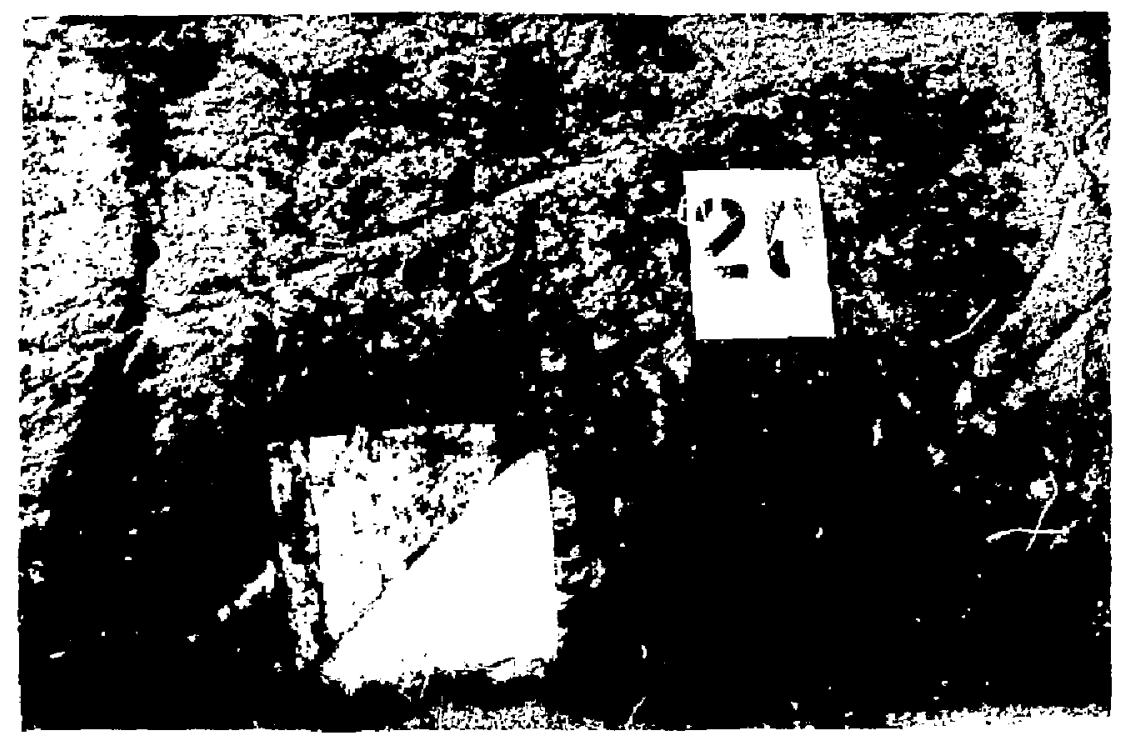

Fig. 126 filter media clogged by smoke aerosols. 
that could tear the filter media, sealing minor leak paths in the reservoirs holding the filter supply and take-up spools, adding an additional rear screen to support the filter medium and extending the blinders used to prevent edge leakage. Figure 127 shows the inlet side of the ralling filter purchased from American Air Filter after these madifications were made.

The filter medium supplied with the REP had a very low efficiency, but could tolerate the tension stresses caused by the pulling force of the takeup reel. This medium a!so had a very low pressure drop due to the large dianeter gla;s fibers and the law fiber-packing density. Another important property of this medium was its low compressibility and spring-like behavior. In order to increase the filter efficiency, we added one or more lajirs of a more efficient filter medium on top of the REP medium to make a laminated system (Fig. 12B). Our laminated system is composed of the folluwing inedia sequence from bottom to top: a $50 \mathrm{~mm}$ layer of the RLP medium, a $6.4 \mathrm{~mm}$ layer of AF-4 medium and a $6.4 \mathrm{~mm}$ layer of Aif -18 medium. The REP medium was a critical component of the laminated structure because it provided the necessary tensile strength for pulling the media through the filter housing and physical support for the higher efficiency media, and also maintained a positive seal for the more efficien' media against the blinders in preventing edge leakage.

\section{Laboratory Evaluation}

He conducted a series of experiments using a rolling prefilter; each enperiment consisted if generating a reproducible fire in the LLML Fire Test Facility and exhausting the smoke through a ventilation duct that containfo the rolling prefilter and HEPA filter. The fire Test facility consists of a negative-pressure ventilation test area, a fire test cell, and a computer diagnostic room. The ventilation test area is coipied to the fire test cell by standaro $2 \mathrm{ft} \times 2$ it ventilation ducting. Diagnostic instrumentation at these locations is connected to a PDP-11 computer for data addressing, data reduction, and final display in hard-copy format.

We used a fuel source made of specific proportions of fuel materials common to physical science laboratories. We ignited the fuel witr. a natural gas burner that acted both as the ignition source and a thermal driver to maintain high, constant temperatures when the ventilation in the test cell was controlled. This burn procedure resu?ted in reproducible fires and smoke 


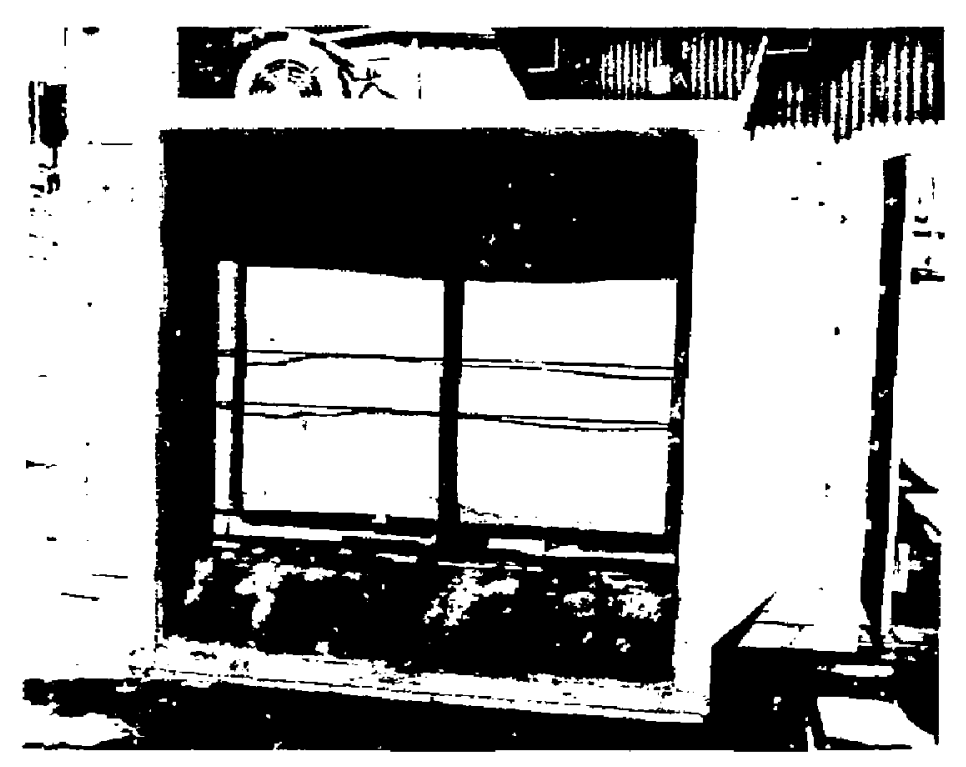

Fig. 127 comercial rolling prefilter modified tor high filter efficiency. 


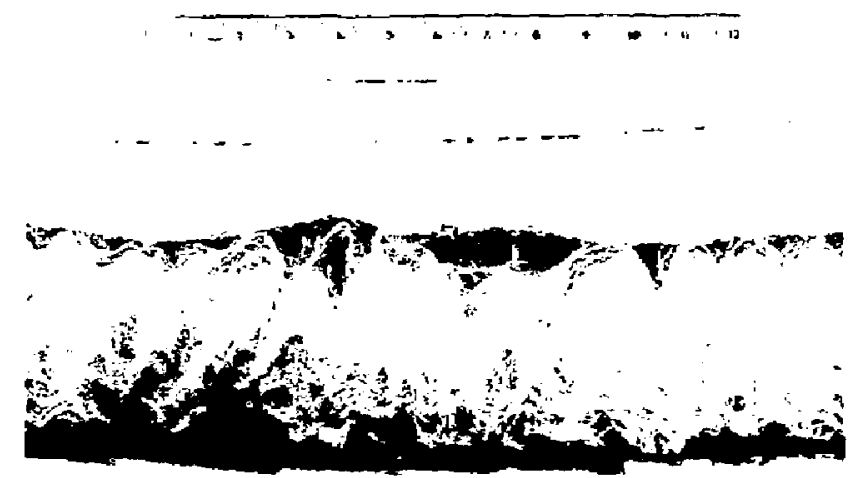

Fig. 128 Laminated filter media used in rolling prefilter. 
aerosols for our filter evaluations. The smoke from the fire test cell was exhausted through a ventilation duct and filtered first by the REP and then by the HEPA filter.

The primary information obtained from these experiments was the effectiveress of the REP to prevent smoke dti,:01s from plugging the HEPA filter. Test results were compared on the basis of the time required for the HEPA filters to plug. Figure 129 shows the pressure drop across the HEPA filter as a function of the burn time for each of six different experiments. A comparison of Tests 1-4 suggest that the REP made little difference in protecting the HEPA filter, although the efficiency of the laminated filter media had steadily increased in the sequerce of Tests 2-4.

We then increased the efficiency of tha laminated media another increment by replacing one of the AF-18 media with the higher efficiency AF-4 media. Test 5 indicates that this combination of filter media was very effective in protecting the HEPA filter from smoke aerosols. The HEPA filter plugged at 130. seconds because we had run out of prefilter media and the HEPA filter was directily exposed to smoke aerosols. We were only abie to insert $9 \mathrm{~m}$ of media on the supply reel because of tine limited space in the modified REP. We then modificd the supply spool 50 that it could accommodate $18 \mathrm{~m}$ of laminated media. The results, shown as Test 6 , indicate that the REP was able to protect the HEPA filter from plugging for the duration of a fire. The fire had seif- inguished and was reduced to a smoldering condition at 3600 seconds, at which time we terminated che test. During this test, we had used 12 in of prefilter media and the pressure drop across the HEPA filter increased from $250 \mathrm{~Pa}$ to $400 \mathrm{~Pa}$.

A more extensive analys is of experiment Test 6 is shown in Fig. 130. This figure shows the pressure drop across the HEPA filter and prefilter and the total air flow rate during the first 2400 seconds of the burn. The oscillations in the pressure drop across the prefilter were due to repeated cycles of filter plugging and filter advancement. Ncte that the pressure fluctuations across the prefilter are also reflected in the flow rate and the pressure drop across the HEPA filter.

Although the single-pass REP is an effective means for nrotecting a HEPA filter from smoke aerosols, its exclusive use as an emergency fire protect: , device is not cost-effective. However, if the REP were designed to also function as a prefilter that extended the life of the HEPA filter under normal 


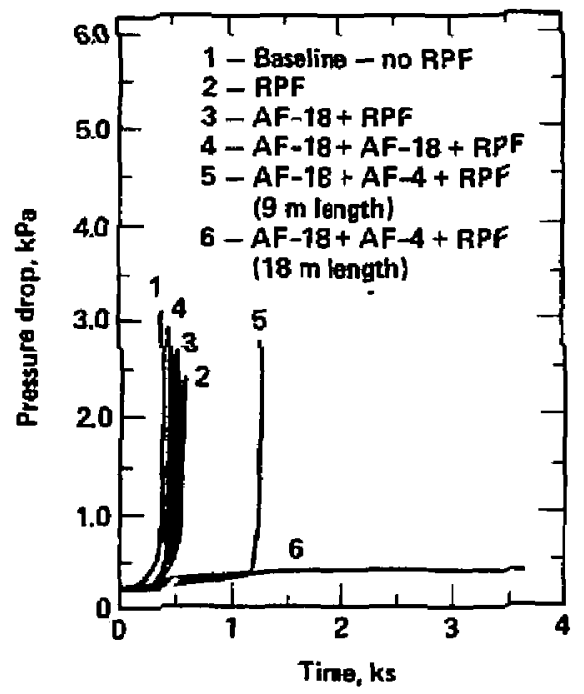

Fig. 129 Effect of filter media on rolling prefilters ability to protect HEPA filter from plugging is smoke aerosols.

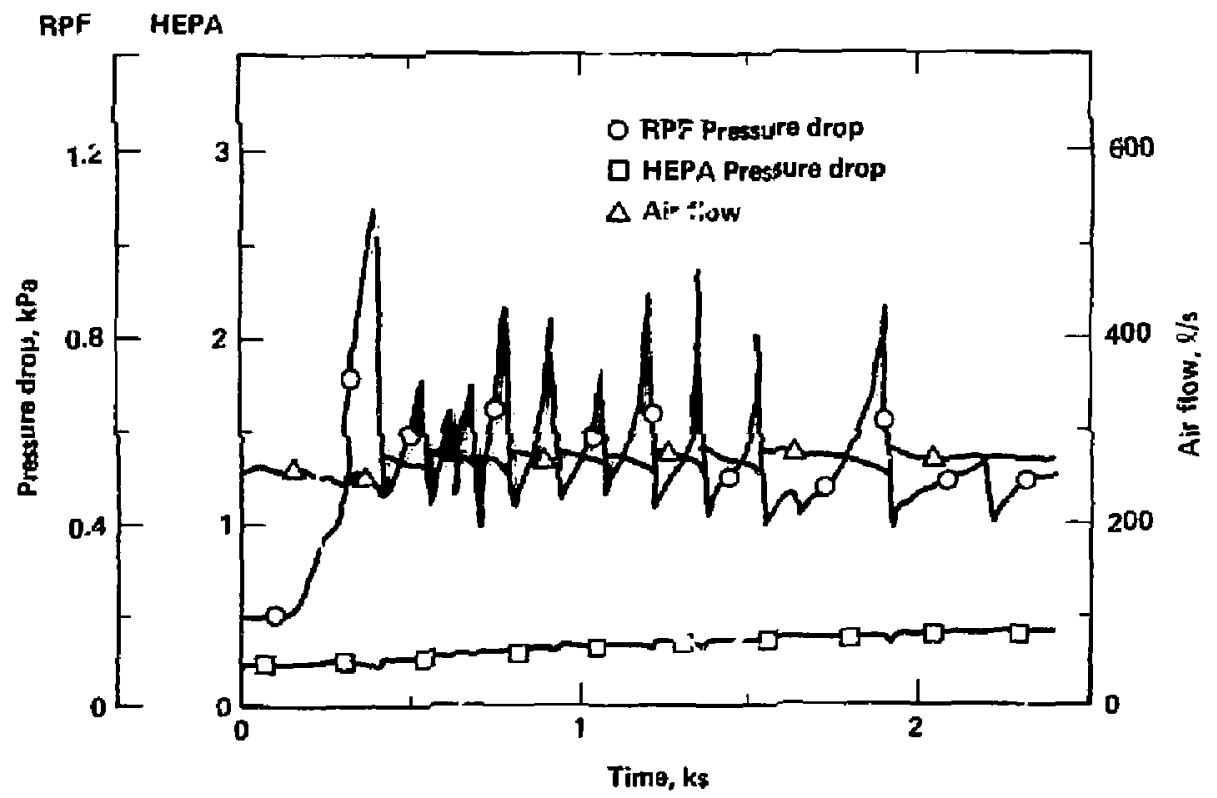

Fig. 130 Performance of the rolling prefilter-HEPA filter system as a function of exposure time to smoke aerosols. 
operating conditions, then tive REP would bacome a cost-effective devico. The only difference between normal and emergency operations would be the iridexing frequency of the prefilter media, which is controlled by its pressure differential. The single-pass REP cannot be used for a conventional prefilter that operates under normal conditions because of the excessive pressure drop that is encountered. The conventional method for reducing the pressire drop is to increase the fitter surface area by pleating the medium. Theref re, we developed a pleated rolling prefilter. In keeping with our terminology for the single-pass REP, we designated this pleated filter a multi-pass REP.

Multi-path REP Design

The starting point in this design was our successful single-" : rEP. The key feature of the single-pass REP which we kept was pulling tic fijter medium over a fixed supporting screen. The pulling force was provided by the take-up reel powered by an electric motor. We changed tour other design fe-tures of the single-pass REP in building the REP shown in Fig. 131. The most dramatic change was replacing the single-pass design with a pleated design to reduce the face velocity. Instead of a single supporting screen across the duct opening, our experimental filter had four screen segments connected to form two $V$-shaped pleats. These screen segments would serve as the high- tage electrode. We also had to add a mating ground electrode.

We installed the electri. REP in our large-scale filter test fac, ty and soon discovered that the design was not practical. Aithough we were able to pull the medium over the pleated electrodes with no air flow, this was not possible at $4721 / \mathrm{s}(1000 \mathrm{cfm})$. The air flow had pressed the medium firmly against tle rear electrode and created an excessive drag. We concluded that the concept of pulling the filter medium over a stat inary, pleated screen was not practical because of the excessive drap on the medium.

Cur next design of the electric REP toak advantage of this high between the filter medium and the rear screen. The key feature here continuous screen belt that provides support for the filter medium. he filter medium rides on the electrude belt and is advanced through th : iter housing as the belt advanced. Figure 132 illustrates the continuous beit that is used in forming the pleated rear screen. The design of the electric iEP is complicated by applying high voitage to the screen belting. Special 


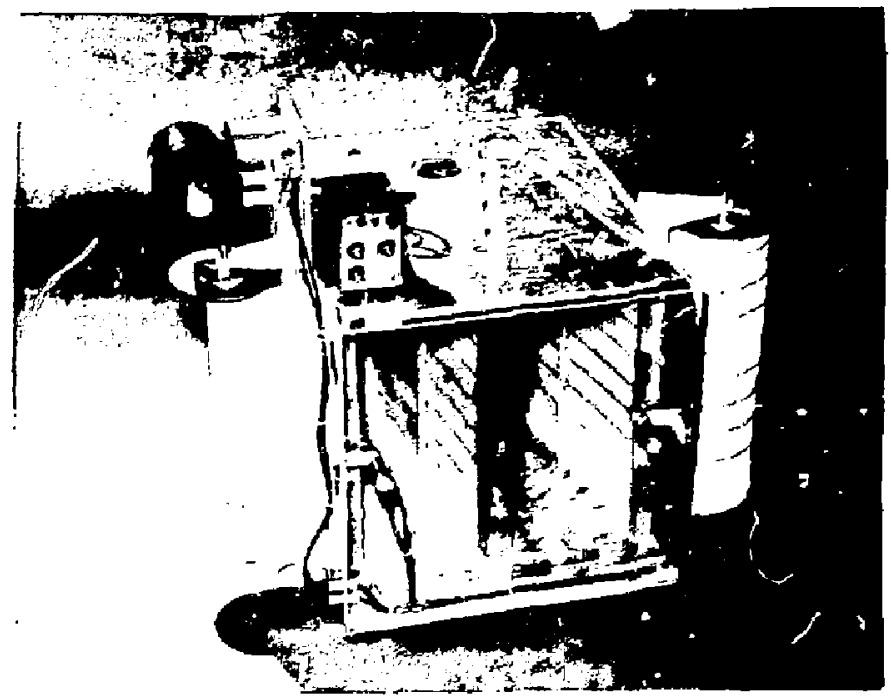

Fig. 131 Electric rolling prefilter having stationary, pleated electrodes. 


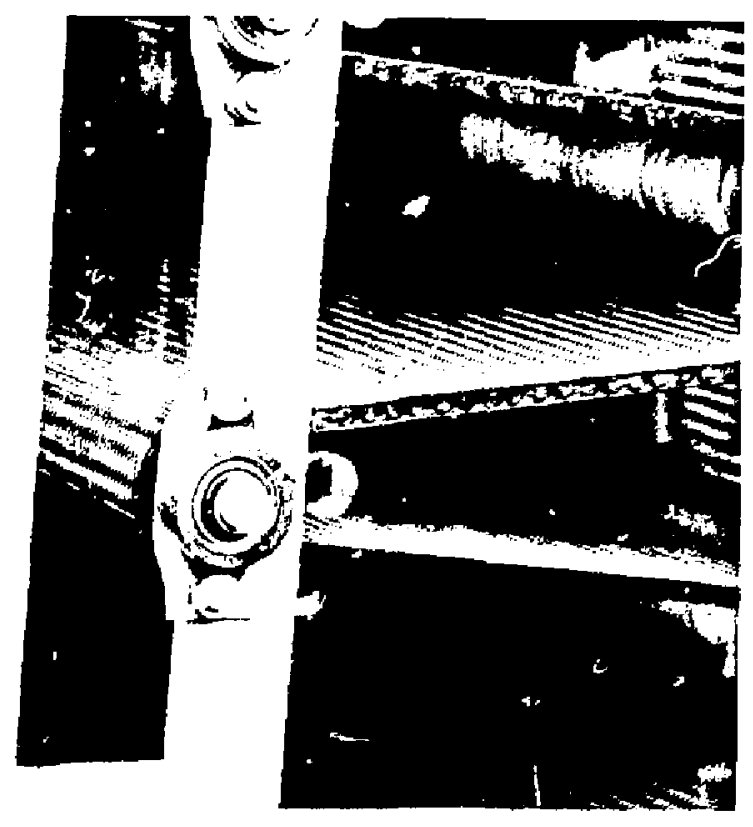

Fig. 132 Continuous screen belting that serves as the nigh voltage e'ectrode and physical support for the filter media. 
precautions are required to ensure adequate insulation for the high-voltage belt. The screen belting is insulated from the REP housing by sheets of polyethylene or polycarbonate with specially cut grooves for supporting the belt chain. In addition to the screen insulation, all connections to the screen belting, like the sprocket shafts, are made from nonconductive material.

The completed electric REP has a roll of AF-18 medium threaded through the unit. The front ground electrode is not installed in this unit because we first wanted to demonstrate the REP's ability to protect HEPA filters from smoke aernsols. Since the high acid and water content of the smuke would short-circuit the electrodes, all of our fire tests were conducted without: the grourt e lectrode (Fig. 133).

We conducted a stries of fire tests to evaluate the ability of the REP to protect HEPA filters from smoke aerosols. Ouring our evaluation tests, we encountered a number of problems that prevented a successful demonstration of the REP to protect tije iEPA filter from alugging due to smoke aerosols. We identified the causes of the test failures, made the appropriate modifications to correct problems and then evaluated the effectiveness of the modifications. This cycle was repeated until all the problems were either eliminated or mitigated and a successful fire test was obtained. The madifications made to the REP were generally dictated by te; $i$ results obtained in our small-scale laboratory experiments using NaCl aerosols. The result is the REP shown in Fig. 134.

An improved REP was constructed using tire improved filter media and edge seal developed previousiy; this took care of leak problens. In operation, the prefitter inlet duct (the central apening in Fig. 134) is connected to the upstream ventilation ducting. Through this opening, the filter media can be viewed traversing the yentilation duct along three $V$-pleats. The lef: and right ports infig. 134 expose portions of the take-up mechanism and storage box for the filter medium respectively. Plexiglas panels can be removed for access to these areas.

The control box located on top of the prefilter housing allows the selection of an appropriate pressure drop across tile prefilter at which the filter media is automatically advanced by the drive mecinanis.n. In this manner. the filter medium, which becomes loaded with par:iculate material, can be renlaced continually with clean medium. The filter medium traverses from the fliter medtum storage box (right side of F1g. 134) through the pleated prefilter sertion (center portion) and is pulled out of the prefilter section 


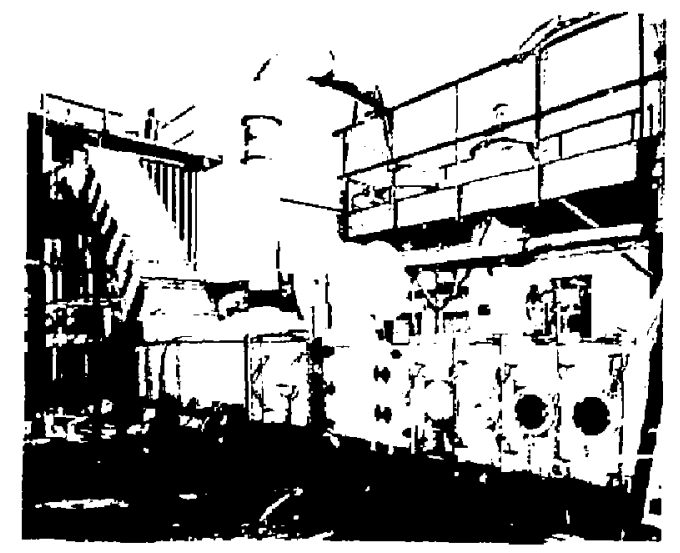

Fig. 13s Rolling electric prefilter installed in che ventilation system of LLNL.

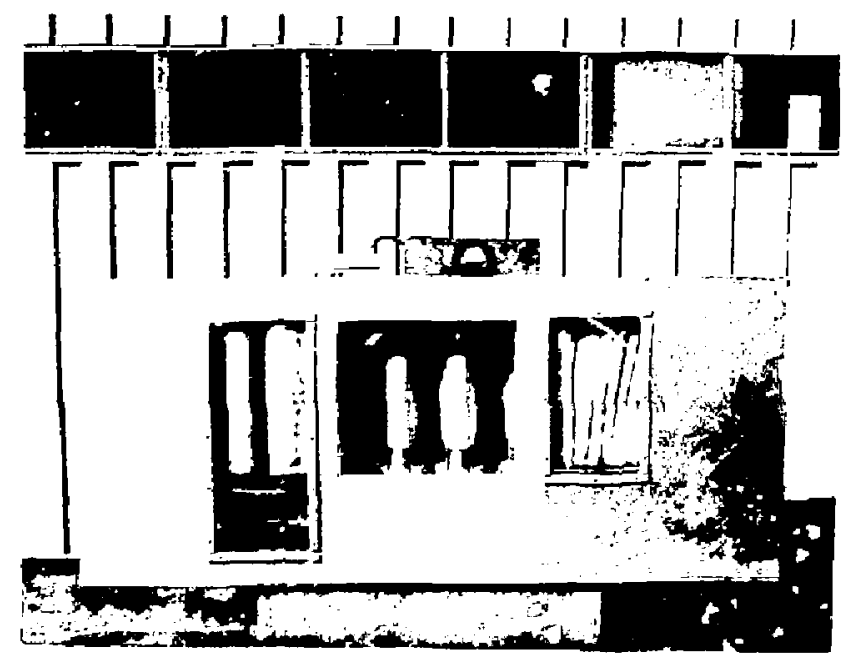

Fig. 134 Improved oesign of rolling eiectric prefilter. 
by take-up rollers (left side of Fig. 134) and finaliy wound on a disposal spool. When the of sposal spool has reached its capacity, the filter medium is cut, and the spoot removed fruiil the disposal box and placed directly into a waste drum. The cut filter medium is then attached to a new spool. A very important feature of our filter design is that fresh filter medium can be added and used fitter medium disposed of while the rolling prefilter is in operation.

We added three take-up rollers on the left side of the filter to pull the media out of the pleated filter section and improve guidance onto the filter medium disposal spool. The arive mechanisms for the take-up rollers and disposal spool are located belaw the disposal spool. The enclosure box has flanges that normally provide support to the filter medium supply.

The central filterirg section of the electric REP is shown in Fig. 135. One of the most $\mathrm{cr}^{\prime \prime} i \mathrm{cal}$ components of the filtering section is the filter edge sealing flange that keeps the filcer media under slight compression to minimize lezks. The central filtering section also has three rollers that drive the filter medium around the rear pleats. These rollers have short blades running along their length to grab the filter medium and drive it through the sharp turns without providing a sticking surface for the filter medium.

The filter we used in the REP is composed of five layers of Johns-Manville filter medium. These include, in order from upstrean to downstream, two layers of AF-21, two layers of AF-4, and one layer of AF-21. The two layers of AF-21 were used on the upstream to provide a high loading capacity for liquid smoke aerosols and good resistance to compression. The two central layers of $4 \mathrm{AF}-4$ were used to make the composite filter medium more efficient. A final layer of AF-1B:as added to the composite filter to protect the AF- 4 filter medium from being torn due to mechanical forces or due to sticking to tarry surfaces. A scrim material is sewn to the filtor medium on both sides to provide more strength to this laminated structure. When the prefilter is 'used in an electrified mode of operation, an additional metal mesh screen (nnt shown) will be glued on the upstream side of the filter medium in place of the scrim material to serve as the ground electrode.

\section{Laboratory Evaluation}

We conducted a laboratory evaluation of the filter medium using DoS aerosols at a f, ie velocity of $22.5 \mathrm{~cm} / \mathrm{s}$. This face velocity corresponds to 


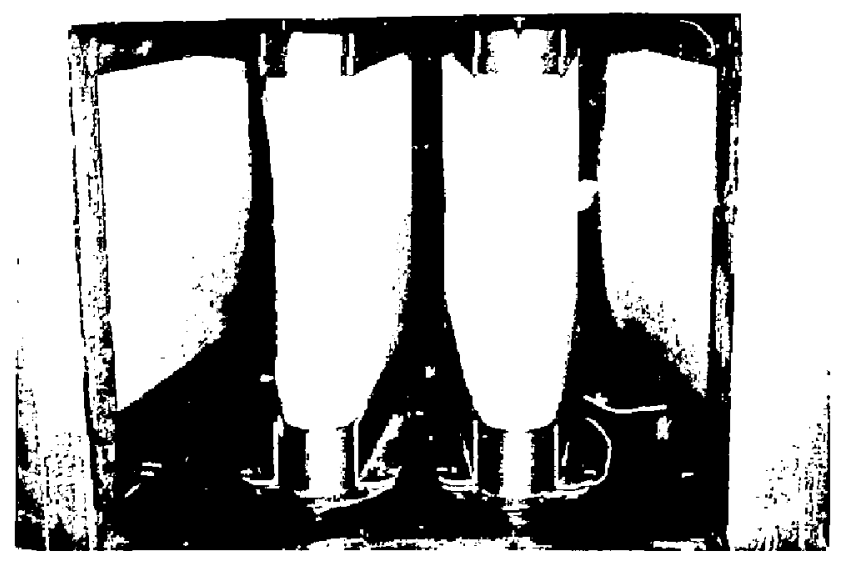

Fig. 135 Central filtering section of rolling electric prefilter. 
$500 \mathrm{cfm}$ through the rolling prefilter unit. Liquid DOS aerosols were usec in this evaluation since smoke aerosols are predominantly liquid. Figure 136 shows the percent penetration of DOS aerosols throunk the composite filter medium as a furction of particle dianieter. This figure shows the percent penetration with and without an applied electric fieid. At a face velocity of $22.5 \mathrm{~cm} / \mathrm{s}$, the filter had a pressure drop of : in. water. Using a lightscattering photometer, we determined that the filter efficiency was $97.5 \%$ without an applied voltage and $99.6 \%$ with $7 \mathrm{kV}$ applied to the electrodes. Electrifying the filter medium had therefore reduced the aerosol penetration by $83 \%$.

However, when the rolling prefilter using the same filter madium was tested under simitar concitions, it had a pressure drop of only $0.6 \mathrm{in}$. and an efficienisy of R4\% with no applied voltage. The low pressure drop in the REP was due to leaks from the upstream to the downstream side of the filter media as well as leaks from outside the roling prefilter to the downytream side of the filter. The leaks from outside the rolling prefilter caused a reduction in the total flow through the REP while leaks from the upstream side of the filter result in a lower filter efficiency. The drop in efficiency from $97.5 \%$ to $84 \%$ is due to edge leakage. A traverse of the particle concentration across the downstieam side of the rolling prefilter showed large increases in particle deposition near both sides of the filter housing. The traverse confirmed that edge leakage is the cause of the low filter efficiency. $f$.suming that the particles follow the leak patri, we estimate the rolling prefilter has a $14 \%$ air leak. The occurrence of such a ifigh leakage, in spite of our elaborate efforts to minimize it, iTlustrates the importance of the sdge-sealing mechanism in the rolling prefilter design,

\section{Evaluation of Electric REP in Dense Smoke Aerosols}

We conducted an evaluation of the REP in the exhaust duct of the fire test facility to study the effectiveness of the REP in preventing the HEPA filter from plugging by snake aerosols. Figure 137 shos:s the test results of placing our REP in the ventilation duct upstream of a HEPA filter after a fuel source was ignited in the fire test cell. We have plotted the pressure drop across the prefilter and HEPA filter as a function of time from the start of the fire. Figure 137 also shows the total air flow through the ventilation duct. 


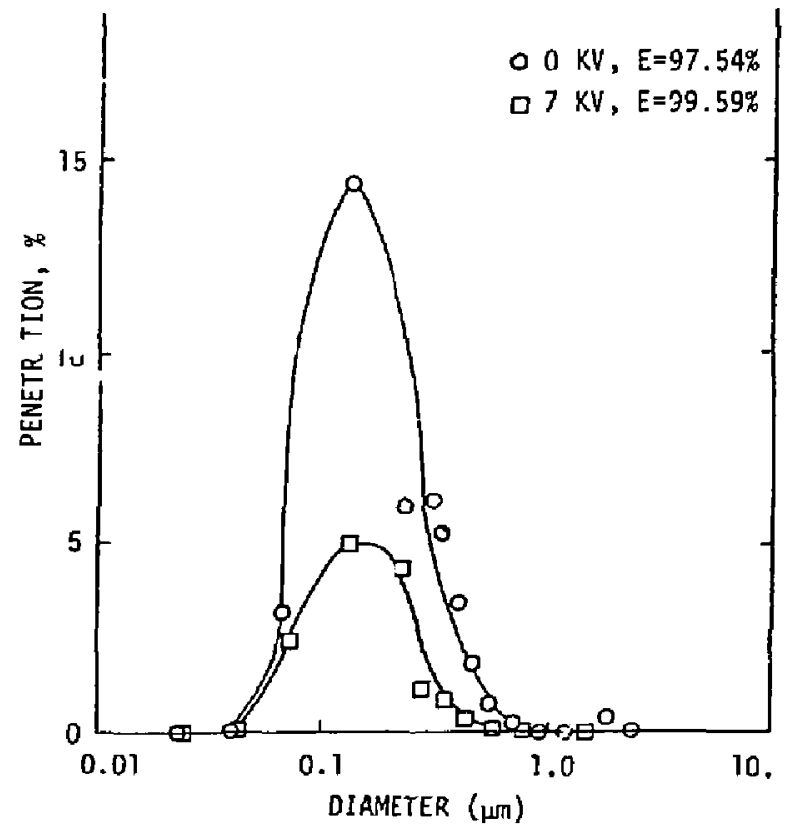

Fig. 136 Penetration of DOS aerosols inrougn composite media with and without nigh voltage as a functicn of partic\}e size. 


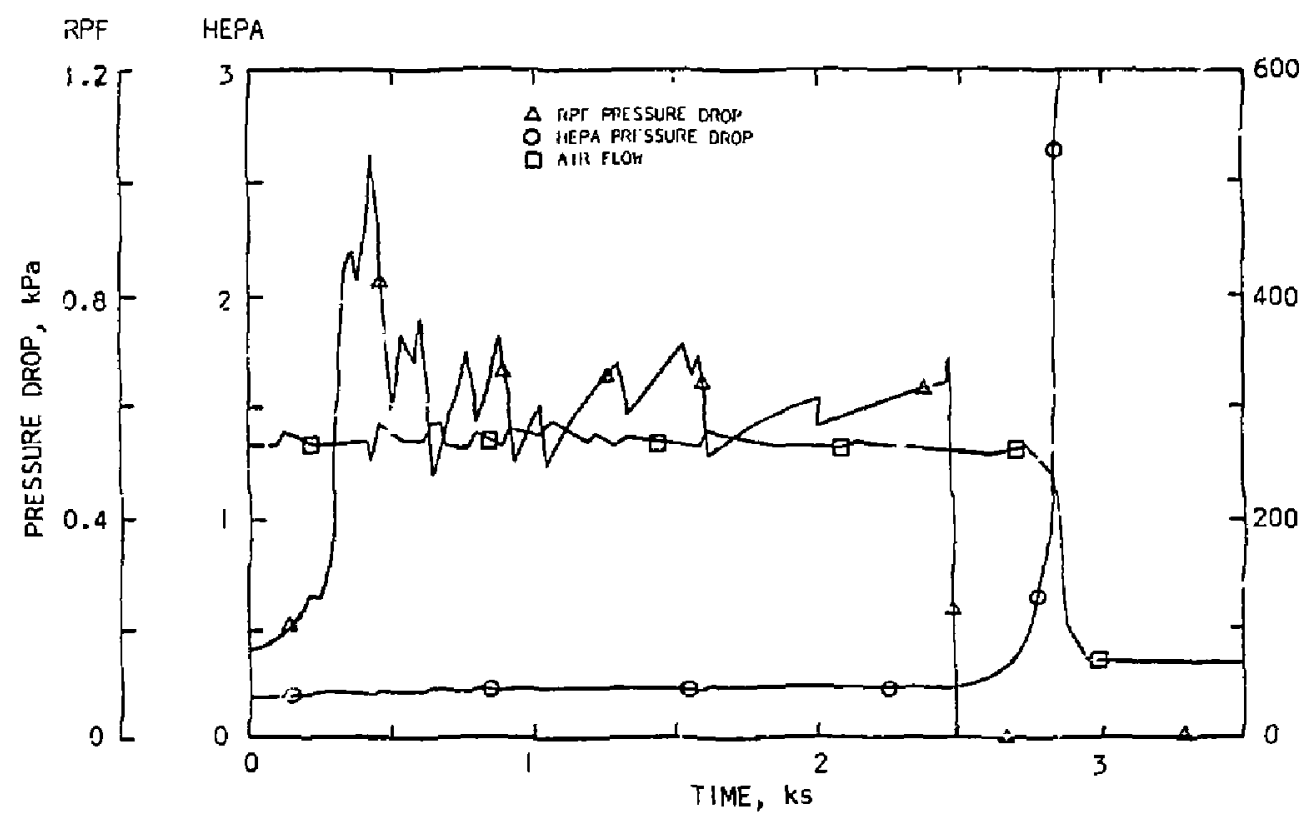

Fig. 137 Performance of the improved rolling alectric prefilter as a function of exposure time to snoke aerosals. 
The primary information obtained in this experiment is the ability of the REP to prevent the snoke aerosols from plugging the downstream HEPA filter. The oscillations in the pressure drop across the prefilter up to $2500 \mathrm{~s}$ are due to repeated cycles of filter plugging and filter advancement. The pressure fluctuations across the prefilter are also somewhat reflected in the air flow rate. In order to assess the effectiveness of the prefilter to prevent the HEPA from clogging, the prefilter media was cut at $2500 \mathrm{~s}$ into the experiment. After this time, the pressure drop across the HEPA begin to increase dramatically, and the HEPA essentially clogged within another $400 \mathrm{~s}$. This test shows the rolling prefilter is very effective in protecting the downstrean HEPA from the smoke aerosol.

\section{ELECTRIC HEPA FILTER}

\section{INTROOUCTION}

Al1 of the experimental electric air filters described so far in this report were designed for use as prefilters to HEPA filters. Hawever, techniques used to electrify the low- and medium-efficiency filters can also be used to electrify high-efficiency filters. In fact, the extremely low filter-face velocity of the air flow in HEPA filters makes them an ideal candidate for electrification. Figure 138 jllustrates the dramatic increase in filter efficiency that occurs as the face velocity decreases, especially at high voltages. Sirice HEPA filters nomally operate at face velocities below $2.5 \mathrm{~cm} / \mathrm{s}, \mathrm{Fig} .138$ shows that converting a standard HEPA fi\}ter to an electric HEPA filter will improve the filter efficiency by 100 times. We should point out that the filter media shown in Fig. 138 was an AF-18 medic that only had an efficiency of $35 \%$ at $16 \mathrm{~cm} / \mathrm{s}$ with no applied voltage. The difference between the lower grade AF-18 media and the high efficiency HEPA media may result in a different degree of electrical enhancement for the HEPA filter.

The electrification of a HEPA filter is a relatively simple task since many HEPA filters already use aluminum separators to maintain the spacing between the pleated filter media. Figure 139 shows how easily a standard HEPA filter can be converted to an elcuric HEPA filter. Since the aluminum separators are inserted in the deep pleats of the filter media, every other 


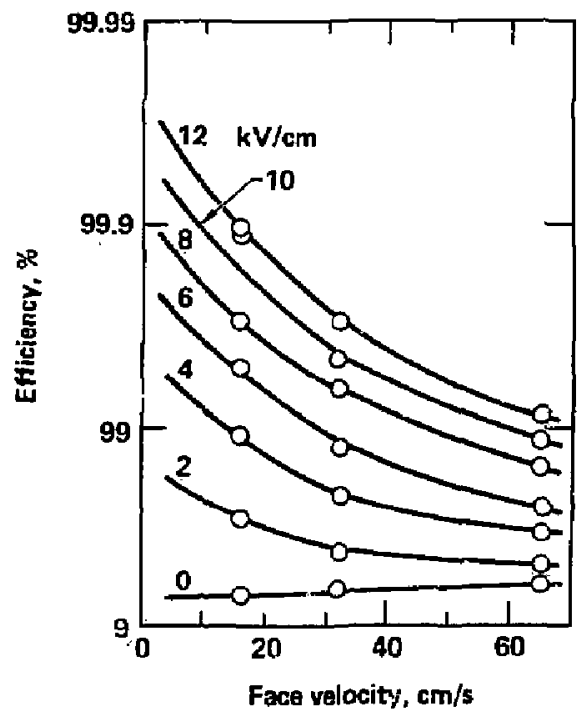

Fig. 138 Filter efficiency as a function of face velocity at various applied vol tages for AF-18 modium.

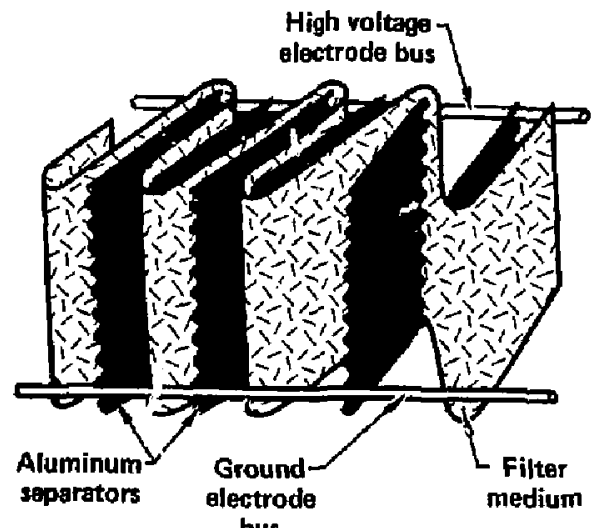

Fig. 139 Schematic showing the canyersion of a conventional HEPA filter to an electric HEPA filter. 
separator will be exposed on one side of the composite filter. These alternate aluminum separators can be connected electrically by a single electrode bus. Grounding the electrode bus on one side of the filter and applying high voltage to the electrode bus on the other side converts the HEPA filter to an electric HEPA filter.

\section{Laboratory Evaluation}

We evaluated the performance of the electric HEPA filter in laboratory tests using heterodisperse $\mathrm{NaCl}$ aerosols and an ASA laser particie counter. The evaluation consisted of measuring the filter efficiency as a function of particle size for the elertric HEPA filter at increasing applied voltages. Unfortunately, we were unable to measure the filter efficiency at applied voltages much greater than $1.25 \mathrm{kV}$ due to electrical sparking. Figure 140 shows the measured filter efficiency as a function of particie size for the electric HEPA filter with an applied electric field of $0 \mathrm{kV} / \mathrm{cm}$ and $1.9 \mathrm{kV} / \mathrm{cm}$. The $1.9 \mathrm{kV} / \mathrm{cm}$ represents an average field strength due to $1.25 \mathrm{kV}$ applied to the corregated electrode separators. Since the spacing between the ground and high voltage electrodes varies widely due to the corregation, an average electrode separation of $0.66 \mathrm{~cm}$ was used in comuting the average electric field strength.

Figure 140 shows that applying $1.9 \mathrm{kV} / \mathrm{cm}$ to the electric HEPA filter dramatically increases the filter efficiency. In fact the filter efficiency with $1.9 \mathrm{kV} / \mathrm{cm}$ at $0.3 \mu \mathrm{m}$ diameter was beyond our experimental detection limit of 99.999\%. The solid curves in Fig. 140 are theoretical filter efficiencies fitted to the experimental data. hlthough we were not able to apply more than $1.9 \mathrm{kV} / \mathrm{cm}$ to the electric filuer, we have shown the notential increase in filter efficiency that occurs with $10 \mathrm{kV} / \mathrm{cm}$.

Electric sparking is a problem that keeps the voltage which can be applied to the filter separators relatively low. This significantly reduces the potential for dramatically increasing the HEPA filter efficiency. In addition to the restriction in the enhancement of filter efficiency, the electric HEPA design is prone to forming pinholes in the filter media at the place where an electric spark occurs. The formation of these pinholes was readily detected by the laser particle counter since the particle concentraiton downstream of the HEPA filter increased dramatically each $t$ ime there was a spark. 


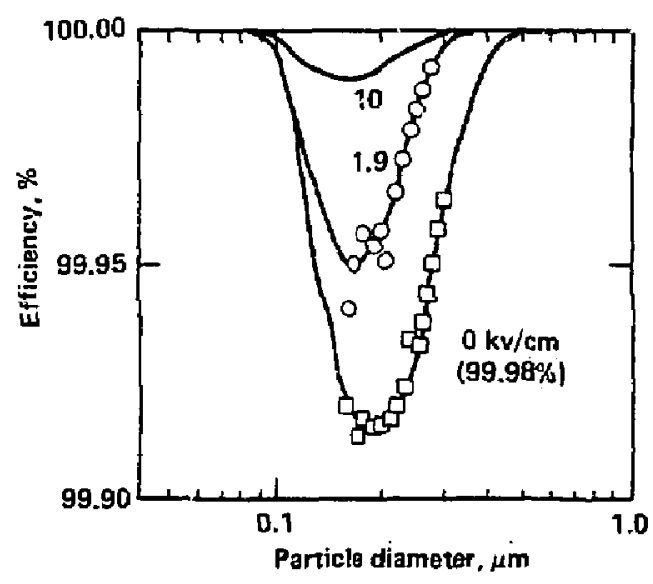

Fig. 140 Filter efficiency for electric HEPA filter measured at 0 and $1.9 \mathrm{kV} / \mathrm{cm}$ and computed for $10 \mathrm{kV} / \mathrm{cm}$. 
The source of the sparking that both limited the degree of electrical enhancement and produced pintole leaks in the HEPA media was the non-uniform spacing between the high voitage and ground electrode separators. There is a great variation in the separation distance between adjacent electrodes. At some locations, the adjacent electrodes are only separated by the thickness of the HEPA media while at other lacations the separation is twice the separation distance of the HEPA media. It is obviaus that applying high voltage to one electri- separator while grounding the adjacent separator will produce sparks at the points of closest contact.

Thus, we see that the very separators that allaw the HEPA filter to be easily converted to an electric HEPA tilter also limits the degree of electrical enhancement. The development of an electric HEPA filter that avoids the sparking problem would require a major modification to the standard HEPA design.

\section{REFERENCES}

1. Davies, C. N., Air Filtration, Academic Press, New York (1973).

2. Hinds W., M. W. First, and D. Leith, "Size Measurement of Narrow Distribution Aerosols by the Polarization Ratio of $90^{\circ}$ Scattered Light," J. Aerosol Science, 10, pp. 437-443 (1979).

3. Tillary M., G. Salzman and H, Ettinger, "The Effect of Particle Size Variation on Filtration Efficiency Measured by the HEDA Filter Duality Assurance Test," Proceedings of the 17th DOE Nuclear Air Cleaning Conference, Denver, Colorado, August 1982, National Technical Information Service Conf-820833, pp. 895-908.

4. Langmuir, I. and K. 8lodgett, "Report on Smokes and Filiers," Supplement to Section ! and Section II. U.S. Office of Scientific Research and Development, No. 3460, (1944).

5. Lee, K. W. and Liu, B.Y.H., "On the Minimum Efficiency and Most Penetrating Particle Size for Fibrous Filters," J. Air Poll. Control Assoc. 30, 377-381 (1980).

6. Wi]leke, K., "Generation of Aerosols and Facilities for Exposure Experiments," Ann Arbor Science Publishers, Inc., Ann Arbor, 1980.

7. Lundgrer, D. A., et al. Aerosol Measurement, University Press of Flo"ida, Gainesville, 1979.

8. R. Dennis, ed., "Handbcok on Aerosols," National Technical Information Service, 1976. 
9. Liu, B.Y.H, Whitbj, K.T, and Yu, H.H.S., "A Condensation Aerosol fenerator for Producing Monodisperse Aerosols in the Size Range 0.03-1.3нm," J. Rech. Atmo 3, 393 (Paris).

10. W. T. Davis, "Air Filter Media Efficiency Testing Utilizing Uniform Latex Spheres," Filtration and Separation 18, fp. 143-147 (1981).

11. K. T. Whitby and B.Y.H. Liu, "Generation of Cointable PuTses by High Concentration of Subcountable Sized Particles in the Sensing Volume of Optica) Counters," J. Colloid and Interface Science, 25, 537-546 (1967).

12. Liu, B. Y. H. and Pui, D. Y. H., "A Submicron Aerosol Standard and the Primary absolute Calibration of the Condensation Nuclei Counter," J. of Colloid and Interf. Sci., 47:155-171 (1974).

13. Biermann, A. H. and Bergman, W., "Measurement of Aerosol Concentration as a Function of Size and Charge, "Submitted to Aerosol Science and Technology, 'iCRL-88477, July 1983.

14. Mazumder, M. K., Kirshc, K. J., "Single Particle Aerodvnamic Relaxation Time Analyzer," Rev. Sci. Instrum. 48, June 1977, pp.622-624.

15. Renninger, R. D., Mazunder, M. K. and Testerman, M. K., "Particle Sizing by Electrical Single Particle Aerodynamic Relaxation Time Analyzer," Rey. Sci. Instrum. 52(2), Feb. 1981, p.242-246.

16. Dennis, R., Billings, C. E. and Silverman, L., "Performance Characteristics of the Model K Electro-Poiar Filter, "Proceedings of 3rd IJ.S. Atomic Energy Comission Nuclear Air Cleaning Conference, Mational Technical Information Service, WASH-170, pp.262-271, (1953).

17. Felthan, F. J., "The Hansen Filter," Filtration and Separators, 16, pp. $370-372(1978)$.

18. Rossano, A. T., and Silverman, L., "Electrostatic Effects in Fiber Filters for Aerosols," Heating and Ventilating, Ref. Sect. 102 (1954).

19. Reid, D. L., and Browne, L. M., "The Electrostatic Capture of Submicron Particles in Fiber Beds," Proc. 14th ERDA Air Cleaning Conf. (Sun Vatley, Id aho, 1976).

20. Mazumder, M. K. and Thomas, K. T., "Improvenent of the Efficiency of Particulate Filters by Superimposed Electrostatic Forces," Filtration and Separation, 25, (1967).

21. Silverman, L., Billings, C. E. and Dennis, R., Performance of the Madel $\mathrm{K}$ Electro-Polar Filter, USAEC Report NYO 1592, Harvard University (T954).

22. Rivers, R. D., "Operating Principies of Non-ionizing Electrostatic Air Filters," d. ASHRAE, 37, (1962).

23. Zebe1, G., 1965, 3. Colloid Sci., 20, 552. 
24. Krisch, A. A., "The Influence of an External Electric Field on the Depnsition of Aerosols in Fiurous Filters," J. Aerosoi Sci , 3,25 (1972).

25. Walkenhorst, $W_{*}$, "Reflections and Research on the Filtration of Dust From Gases with Special Consideration of Electrical Forces, "J. Aersol Sci., I, $225(1970)$.

26. Iinoya, K. and Makina, K., "Applications of Electric Field Effects to Dust Collection Filters," J. Aerosol Sci., 15, 357 (1974).

27. Bogardus, H. F., Clark, R. C., Thompson, J. K. and Fielding, G. H., "Enchancement of Filter Media Performance by Corona-free Electric Fields, " Proc. 13th AEC Air Cleaning Conf. (San Francisco, Calif., 1974).

28. Lamb, G. E. R. and Costanza, P. A., "Electrical Stimulation of Fabric Filtration," S. Textile Res., 372 (1977).

29. Ariman, T. and Tank, L., "Collection of Aerosol Particles by Fabric Filters in an Electrostatic Field," Atmos. Envir In 205 (1976).

30. Nielsen, K. A. and HilT, J, C., "Collection of Inertialess Particles on Spheres with Electrical Forces," Ind. Eng. Chem., Fundam. 15 149 (1976).

31. Frederick, E. R., "Some Effects of Electrostatic Charges in Fabric Filtration," Fine Particulate Control Technology, H. M. Englund and W. T. Beery eds., Air Pollution Control Assocation, Pitsburgh, PA (1975).

32. Penney, G. W., "Using Electrostatic Forces to Reduce Pressure Drop in Fabric Filters," Powder Tech. 18, 111 (1977).

33. Spielman, L. and Gorer, S. L. "Model for Predicting Pressure Drop and Filtration Efficiency in Fibrous Media," Environ. Sci. Tech. 2, p. 279 1968).

34. Nielsen, K. A. and Hill, J. C., "Particle Chain Formation in Aerosol Filtration with Electrical Forces, Am. Inst. Chem. Eng. J., Vol. 26, pp. 678-680, 1980 .

35. Pich, J., Environ. Sci. Techn. 11, p. 608 (1977).

36. Lamb, G. E. R., Constanza, P. A., D'Meara, D. J. (1978), J. Textile Res., p. 566 .

37. Bergman, W., Taylor, R. D., Miller, H. H., Biermann, A. H., Hebard, H. D., da Roza, R. A. and Lum, B. Y., 1978, "Enchanced Filtration Program at LLNL. - A Progress Report," 15th DOE Air Cleaning Conf. Proc., Boston, Massachusetts.

38. Thomas, J. W. and Hoodfin, E. J., "Electrified Air Filters," ‥ AIEE, 275 (1979).

39. Inculet, I. 1. and Castle, G. S. P., "A iwo-Stage Concentric Geometry Electrostatic Precipitator with Electrified Media," J. ASHRAE, 47, (1971). 
40. Van Turnhout, J., "The Use of Polymers for Electrets," J. of Electrostatics, 1, pp. 147-163 (1975).

41. Van Turnhout, J., Albers, J. H. M., Adamse, J. W. C., Hoeneveld, W. J. ants Visscher, J., "Electret Filters for High-Efficiency Air Cleaning," in Proceedings of the Second World Filtration Congress, Sept. 18-20, 1979, London, England. Jplands Press Ltd., Croyden, England, pp. 521-528 (1979).

42. Bergman, W., Hebard, H., and B. Lum., "Electrostatic Filters Generated by Electric Fields," Proc. Second World Filtration Congress, Inpaged, London, 3979.

43. Kuwabara, S., "The Forces Experienced by Randomiy Distributed Parallel Circular Cylinders or Spheres in Viscous Flow at Small Reynolds Number," J. Phys. Soc. Japan, 14, 527 (1959).

44. Zebel, 6., J. Colloid Interface Sci., 27, 194 (1068).

45. Billings, C. E. and Gussman, R. A., "Dynamics Behavior of Aerosols, Chap. 3, Handbook on Aerosols," R. Dennis, Ed., National Technical Information Service, T976.

46. Yeh, H. and Liu, B. Y. Y., 1. Aerosols Sci, $\underline{5}$, p. 191 \{1974\}.

47. Banks, O. and Hall, M., "The Numerical Determination of Single Fiber Efficiency with Electric Enhancement," LLML Reprt., UCRL-84832.

49. Banks, 0. 0. and Kunowksi, G. J., "A Perturbation Method for the Approximation of the Inertial Collection Efficiency for Fibrous Filters with Electrical Enhancement," J. Aerosol Sci. (Accepted for Publication).

49. Stechking, I., Kirsh, A. and Fuchs, N., Ann. Occup. Hyg. 12, p. 1 (1969).

50. Brown, R, C.. "Electrical Effects in Dust Filters," in Proceedings of the Ser.and World Filtration Congress, Sept. 18-20, 1979. London, England. Uplinds Press Ltd., T Katharine Street, Croyden, CRg ILB, England, PP. 291-301.

51. Nelson, G. O., Bergman, W., Miller, H. H., Taylor, R. O., Richards, C. P., and Biermann, A. H., "Enhancement of Air Filtration Using Electric

Fieids," Am. Ind. Hyg. Assoc. J., 39 pp. 472-479 (1978).

52. Biermann, A. H., Lum, B. Y. and Bergman, W., "Evaluation of Permanently Charged Electrofibrous Filters," Presented at the 17th DOE Nuclear Air Cleaning Conference, Denver, co, August 2-5, 1982.

53. Payatakes, A. C., "Advances in Dendritic Deposition of Aerosols by Inertial Inpaction and/or Interception," Proc. Second Wor?d Filtration Congress, pp. 507-519, 1979.

54. Billings, C. E., "Effects of Particle Accumulation in Aerosol Filtration, Ph.0. Dissertation, Cal. Inst. Tech., Masadena, CA, 1966. 
55. Bhutra, S. and Payatakes, A. C., "Experimental Investigation of Dendritic Deposition of Aerosol Particles, J. Aerosol Science, Vol. 10, pp. 445-464, โ979.

56. Barto, D. T., Tien, C. and Wang, C., "Accumulation of Solid Particles on Single Fibers Exposed to Aerosol Flows, Am. Inst. Chem. Eng. J., Vol. 26, pp. 2890-292, 1980.

57. Lamb, G. E. R. and Costanza, P. A., "A Low Energy Electrified Filter System, Filtration and Separation, Vol. 4, 1980 .

5B. Bergman, W., B iermann, A. H., Hebard, H. D., Lum, B. Y. and Kuhl, W. D., "Electrostatic Air Filters Generated by Electric Fields," LLNL Report, UCRL-84878, Jantuary 27, 1981.

:9. Nielsen, K. A., "Coliection of Inertialess Particles on Circular Cylinders with Electrical forces and Graviation, J. Colloid and Interface Sci., Vol. 64, pp. i3]-142, i978.

60. Wang, C. S., Ho, C. P., Makino, H. and Iinoya, K., "Effect of Electrustat ic Fields on Accumulation of Solid Particles on Single Cylinders," An. Inst. Chem. Eng. 3., Vo 1. 26, pp. 680-683, 1980.

51. Hochrainer, D.. Hidy, G. M. and Zebe1, G., "Creeping Motion of Charged Particles Around a Cylinder in an Electric Field, J. Colloid and Interface Sci., Vol. 30, D. . 553-567, 1969.

62. Davies, C. N., "The Separation of Airborne Dust and Particles," Proc. Inst. flech. Eng. 13, 185 (1952).

63. Adley, F. E. and Wisehart, D. W., "Fitcer Loading Tests on Certain FiTter Media," 7th AEC Air cleaning Conf. Proc., Office of Technical Services, Washington, 1961.

64. Havlicek, V. " The Improvement of Efficiency of Fibrous Dielectric Filters by Application of an External Electric Field, "Int. J. Air and Water Poll., 4 , 225 (1961).

65. Kuh], W. D., Bergman, W., Biernann, A. H. and Lum, B. Y., "Field Evaluation of Prototype Electrofibrous Filters," UCID-19586, Lawrence Livermore National Laboratory, Sept. 30, 1982. 